University of Rhode Island

DigitalCommons@URI

Open Access Master's Theses

2014

\title{
MODAL EXCITATION OF A FLEXIBLE CYLINDER UNDERGOING VORTEX-INDUCED VIBRATIONS
}

Harrison Zimmer

University of Rhode Island, harrison_zimmer@my.uri.edu

Follow this and additional works at: https://digitalcommons.uri.edu/theses

\section{Recommended Citation}

Zimmer, Harrison, "MODAL EXCITATION OF A FLEXIBLE CYLINDER UNDERGOING VORTEX-INDUCED VIBRATIONS" (2014). Open Access Master's Theses. Paper 323.

https://digitalcommons.uri.edu/theses/323

This Thesis is brought to you for free and open access by DigitalCommons@URI. It has been accepted for inclusion in Open Access Master's Theses by an authorized administrator of DigitalCommons@URI. For more information, please contact digitalcommons-group@uri.edu. 
MODAL EXCITATION OF A FLEXIBLE CYLINDER UNDERGOING VORTEX-INDUCED VIBRATIONS

BY

HARRISON P. ZIMMER

A THESIS SUBMITTED IN PARTIAL FULFILLMENT OF THE

REQUIREMENTS FOR THE DEGREE OF

MASTER OF SCIENCE

IN

OCEAN ENGINEERING

UNIVERSITY OF RHODE ISLAND

2014 
MASTER OF SCIENCE THESIS

$\mathrm{OF}$

HARRISON P. ZIMMER

APPROVED:

Thesis Committee:

$\begin{array}{ll}\text { Major Professor } & \text { Jason M. Dahl } \\ & \text { D.M.L Meyer } \\ & \text { Sau-Lon James Hu } \\ & \text { Nasser H. Zawia } \\ & \text { DEAN OF THE GRADUATE SCHOOL }\end{array}$

UNIVERSITY OF RHODE ISLAND

2014 


\begin{abstract}
Vortex-induced vibration (VIV) is a key issue in ocean engineering design. Numerous deep water structures can experience VIV when exposed to marine currents due to the shedding of vortices downstream of the structure, causing vibration in both the in-line and cross flow directions. This thesis presents an experimental approach that models the motions association with the excitation of a marine riser by considering the combined in-line and cross flow motions excitation of a flexible cylinder.

Under the lock-in phenomena, where the vortex shedding frequency approaches the effective natural frequency, large motion amplitudes can be observed. The vibration of a flexible structure due to VIV is related to the natural frequency of the structure (time dependence) and mode shapes (spatial frequency dependent) associated with the natural frequency. Beams, designed to become excited at the first, second and third mode in the in-line direction, were molded into a flexible cylinder with a length of $43.18 \mathrm{~cm}$. High speed cameras captured image sequences over the period of 12 seconds at 250 frames per second. Using motion tracking software, both the in-line and transverse amplitudes can be displayed by plotting orbital patterns. The assumption that mode shape can influence the motions of a flexible cylinder undergoing VIV is examined.

The fundamental theme throughout this work has been the effect of three different mode shapes in the in-line direction on a flexible cylinder in a free stream. The design of a symmetric mode (first and third) in the in-line direction produced regular and periodic patterns along the entire length of the cylinder for each flow speed tested. However the observations of the cylinder with the 1-2 mode ratio phenomena produced a series of unique trajectories including figure-eight, crescent, and teardrop orbital trajectories. It is shown that for the low mode-
\end{abstract}


number beams tested there are three primary responses possible for the cylinder when trying to excite second mode in the in-line direction and the first mode in the cross-flow direction. This study shows that when a symmetric mode shape is designed, regular patterns are produced. When an asymmetric mode shape is designed to be excited, three unique patters are seen (a) repeatable patterns with teardrop shape,(b) repeatable patterns with figure-eight shape, and (c) random unrepeatable patterns. It was observed that the first mode was being excited in the in the in-line and cross-flow directions and the effective frequencies would remain close to each other. During the transition period between orbital patterns, a 1:2 ratio as seen in the first and third cylinders was witnessed. Random and unrepeatable patterns only occurred when the second mode was excited in the cross-flow direction. 


\section{ACKNOWLEDGMENTS}

It all starts and ends with family, who have shown me that no matter what, they have my back. My parents Libby and Lou supported me throughout this project and never stopped asking questions. I would like to thank my brother for rekindling a sibling rivalry, for which I am grateful. Tony, Marilyn and Marley, though new to the family, have always encouraged me along the way. My major adviser, Professor Jason Dahl has shown enormous patience, support and insight. I wish him the best of luck, and hope that the work performed in this thesis provides a stepping stone for continued studies in the field of vortex-induced vibrations. I would like to thank Professor Cheryl Wilga for the use of her lab, equipment, and most of all, her time. Both Cheryl and Jason have begun an amazing partnership, and I am happy to have had a part. I would like to acknowledge Professor SauLon James Hu and Professor D.M.L. Meyer for agreeing to be on my committee, providing new insight and constructive criticism that helped focus my thoughts. Finally, I would like to acknowledge the various staff, graduate students, and friends that were involved in coding, analyzing data, or lending moral support: Ergsun Gedikli, Joe Gomez, Dan Iwanski, Luke Logan, Gail Paolino, Fred Pease, Brandon Reichl, Alex Stott, and my colleagues at Meridian Ocean Services. 


\section{TABLE OF CONTENTS}

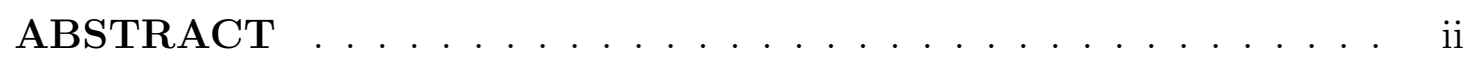

ACKNOWLEDGMENTS .................. iv

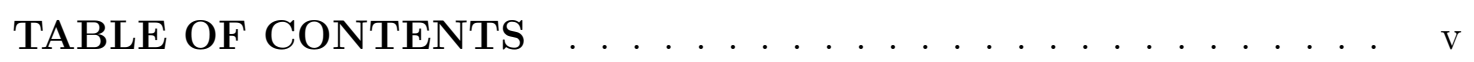

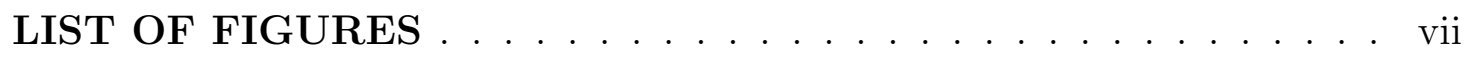

\section{CHAPTER}

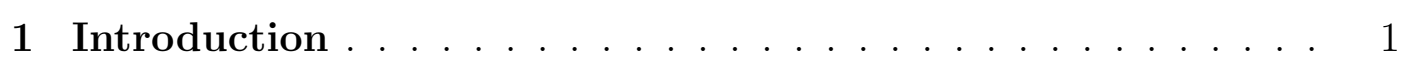

1.0.1 Research Motivation . . . . . . . . . . . . . . 1

1.0.2 Chapter Preview . . . . . . . . . . . . . . 3

2 Vortex Induced Vibrations - Fluid Properties . . . . . . . . . 4

2.1 Vortex Shedding . . . . . . . . . . . . . . . . . . . . 4

2.1.1 Vortex-Induced Vibrations . . . . . . . . . . . 5

2.1.2 Relevant Parameters for Analysis of Vortex Shedding and

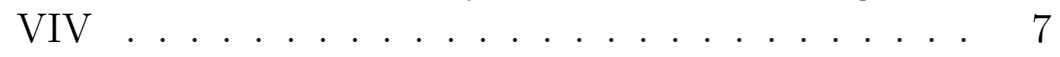

2.2 Literature Review . . . . . . . . . . . . . . . . 8

2.2.1 Rigid Cylinders: One Degree of Freedom (Transverse Only) 8

2.2.2 Rigid Cylinders: One Degree of Freedom (In-line Only) · 10

2.2.3 Rigid Cylinders: Two Degrees of Freedom . . . . . . . 10

2.2.4 Discussion on Orbital Patterns . . . . . . . . . . . . . 11

2.2.5 Flexible Circular Cylinders . . . . . . . . . . . . . 15

3 Flexible Cylinder in Water Tunnel . . . . . . . . . . . . . 20

3.1 Experimental Motivation . . . . . . . . . . . . . . 20 


\section{Page}

3.2 Experimental Procedure . . . . . . . . . . . . . . . . . 22

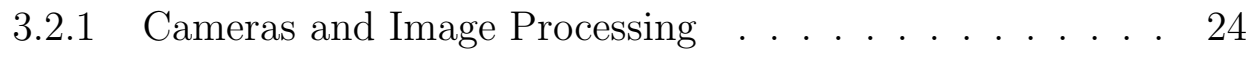

3.3 Results . . . . . . . . . . . . . . . . . . . . 26

3.3.1 Excitation of the First In-line Mode . . . . . . . . . . 26

3.3.2 Excitation of the Second In-line Mode . . . . . . . . . . 33

3.3.3 Excitation of the Third In-line Mode . . . . . . . . . 41

3.4 Discussion on Flexible Cylinder Experiments . . . . . . . . . . 43

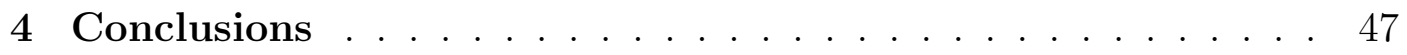

4.1 Overview . . . . . . . . . . . . . . . . . . . 47

$4.1 .1 \quad$ 1-1 Mode Ratio . . . . . . . . . . . . . . . . 47

$4.1 .2 \quad 1-2$ Mode Ratio . . . . . . . . . . . . . . . . . . . . 48

$4.1 .3 \quad 1-3$ Mode Ratio . . . . . . . . . . . . . . . . . . . . . 49

4.2 Future Work . . . . . . . . . . . . . . . . . . . . . . . . 49

LIST OF REFERENCES . . . . . . . . . . . . . . . . . . 51

\section{APPENDIX}

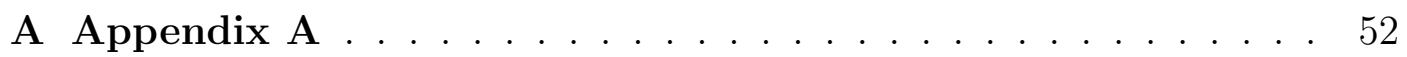

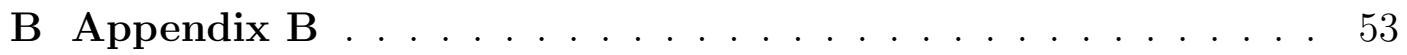

C Appendix C ...................... 73

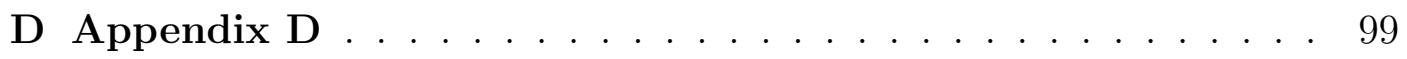

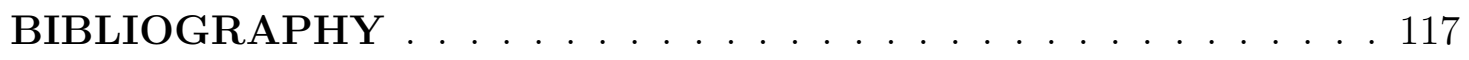




\section{LIST OF FIGURES}

Figure

Page

1 Image modified by Dahl from picture by S. Taneda in Van Dyke 5

2 One Dimension Spring-Pot System Dahl, 2008 . . . . . . . 8

3 Two Dimensional Spring-Pot System . . . . . . . . . . . . 10

4 Cylinder orbital trajectories ............... 11

$5 \quad$ Cylinder orbital trajectories overlaid on the third harmonic magnitude of the lift force. Blue - Trajectory moves downstream at the top of the figure-eight (C: Clockwise); black - trajectory moves upstream at the top of figure-eight (CC: Counterclockwise); flow is left to right. Contours indicate the third harmonic lift coefficient magnitude over the total lift coefficient magnitude. Darkest green denotes a third harmonic magnitude less than 25 percent of the total lift; lightest yellow denotes a third harmonic magnitude greater than 75 percent of the total lift. Dahl et al 2007 . . . . . . . . . . . . . . . . 14

$6 \quad 1-2$ In-line and Transverse Modes . . . . . . . . . . . . . . . . . 17

7 1-1 In-line and Transverse Modes . . . . . . . . . . . . . 23

$8 \quad$ 1-3 In-line and Transverse Modes . . . . . . . . . . . . . . . 23

9 Cylinder Design . . . . . . . . . . . . . . . 23

10 Camera Setup . . . . . . . . . . . . . 25

11 First In-line Mode: A/D vs Vr Present Study Only . . . . . . . 27

12 First In-line Mode: A/D vs Vr. Sarpkaya - Green, Dahl - Red . 28

$13 \mathrm{Vr}=6.16$ Top left - raw data (cross flow), rop right - FFT, bottom left - A/D as a function of Vr, bottom right - orbital pattern for $1-3$ seconds . . . . . . . . . . . . . . . . . . 29

$14 \mathrm{Vr}=6.16$ Time history of amplitude and PSD of the CF Direction 30

$15 \mathrm{Vr}=6.16$ Time history of amplitude and PSD of the IL Direction 30 
$16 \mathrm{Vr}=5.74$ Top left - raw data (cross flow), rop right $-\mathrm{FFT}$, bottom left - A/D as a function of Vr, bottom right - orbital pattern for $1-3$ seconds . . . . . . . . . . . . . . . . 31

$17 \quad \mathrm{Vr}=5.74$ Time history of amplitude and PSD of the CF Direction 31

$18 \quad \mathrm{Vr}=5.74$ Time history of amplitude and PSD of the IL Direction 32

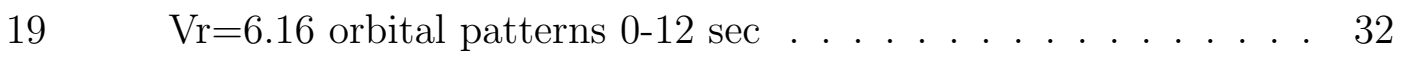

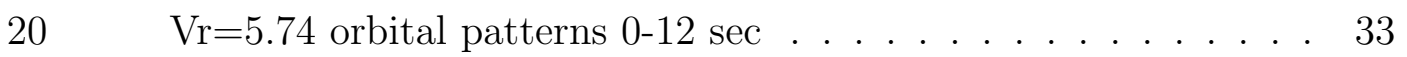

21 Second In-line Mode: A/D vs Vr Present Study Only . . . . . . 34

$22 \operatorname{Vr}=6.13$ Time history of amplitude and PSD of the CF Direction 35

$23 \mathrm{Vr}=6.13$ Time history of amplitude and PSD of the IL Direction 35

$24 \operatorname{Vr}=6.02$ Time history of amplitude and PSD of the IL Direction 36

$25 \mathrm{Vr}=5.9$ Spanwise shape of in-line (Black) and cross-flow (Blue) directions . . . . . . . . . . . . . . . . 37

$26 \quad \mathrm{Vr}=5.9$ orbital patterns $0-12$ sec . . . . . . . . . . . . . 37

$27 \quad \mathrm{Vr}=5.9$ Time history of amplitude and PSD of the CF Direction 38

28 Vr=5.9 Time history of amplitude and PSD of the IL Direction 38

$29 \mathrm{Vr}=5.08$ Spanwise shape of in-line (Black) and cross-flow (Blue) directions . . . . . . . . . . . . . . . . . . . . . 39

$30 \mathrm{Vr}=5.08$ Time history of amplitude and PSD of the CF Direction 39

$31 \mathrm{Vr}=5.08$ Time history of amplitude and PSD of the IL Direction 40

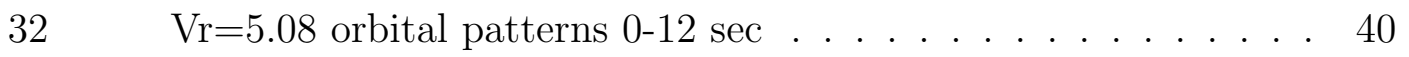

33 Third In-line Mode: A/D vs Vr Present Study Only . . . . . . . 41

34 Comparison of A/D vs $V_{r}$ for all three cylinders. Blue - Excitation of the first IL mode. Green - Excitation of the second IL mode. Red - Excitation of the third IL mode . . . . . . . . . . 42

$35 \mathrm{Vr}=5.25$ Time history of amplitude and PSD of the CF Direction 43 
$36 \mathrm{Vr}=5.25$ Time history of amplitude and PSD of the IL Direction 43

$37 \quad$ Non-dimensional amplitudes and orbit shapes for flexible beam cylinder motions at the midpoint of the beam Dahl(2008). . . . 44

B.1 $\operatorname{Vr}=6.1(\mathrm{U}=0.50 \mathrm{~m} / \mathrm{s})$, Spanwise shape of in-line (Black) and cross-flow (Blue) directions . . . . . . . . . . . 53

B.2 $\mathrm{Vr}=6.1(\mathrm{U}=0.50 \mathrm{~m} / \mathrm{s})$, Time history of amplitude and PSD of the CF Direction . . . . . . . . . . . . . . . . 53

B.3 $\mathrm{Vr}=6.1(\mathrm{U}=0.50 \mathrm{~m} / \mathrm{s})$, Time history of amplitude and PSD of the IL Direction . . . . . . . . . . . . . . . . . . . . . . 54

B.4 $\mathrm{Vr}=6.1(\mathrm{U}=0.50 \mathrm{~m} / \mathrm{s})$, orbital patterns $0-12 \mathrm{sec} \ldots \ldots . . .54$

B.5 $\mathrm{Vr}=6.09(\mathrm{U}=0.51 \mathrm{~m} / \mathrm{s})$, Spanwise shape of in-line (Black) and cross-flow (Blue) directions . . . . . . . . . . . 55

B.6 $\mathrm{Vr}=6.09(\mathrm{U}=0.51 \mathrm{~m} / \mathrm{s})$, Time history of amplitude and PSD of the CF Direction . . . . . . . . . . . . . . . . 55

B.7 $\mathrm{Vr}=6.09(\mathrm{U}=0.51 \mathrm{~m} / \mathrm{s})$, Time history of amplitude and PSD of the IL Direction . . . . . . . . . . . . . . . . . . 56

B.8 $\operatorname{Vr}=6.09(\mathrm{U}=0.51 \mathrm{~m} / \mathrm{s})$, orbital patterns $0-12 \mathrm{sec} \ldots \ldots$

B.9 $\mathrm{Vr}=6.17(\mathrm{U}=0.53 \mathrm{~m} / \mathrm{s})$, Spanwise shape of in-line (Black) and cross-flow (Blue) directions . . . . . . . . . . 57

B.10 $\mathrm{Vr}=6.17(\mathrm{U}=0.53 \mathrm{~m} / \mathrm{s})$, Time history of amplitude and PSD of the CF Direction . . . . . . . . . . . . 57

B.11 $\mathrm{Vr}=6.17(\mathrm{U}=0.53 \mathrm{~m} / \mathrm{s})$, Time history of amplitude and PSD of the IL Direction . . . . . . . . . . . . . . . . 58

B.12 $\operatorname{Vr}=6.17(\mathrm{U}=0.53 \mathrm{~m} / \mathrm{s})$, orbital patterns $0-12 \mathrm{sec} \ldots \ldots$

B.13 $\mathrm{Vr}=5.89(\mathrm{U}=0.55 \mathrm{~m} / \mathrm{s})$, Spanwise shape of in-line (Black) and cross-flow (Blue) directions . . . . . . . . . . . 59

B.14 $\mathrm{Vr}=5.89(\mathrm{U}=0.55 \mathrm{~m} / \mathrm{s})$, Time history of amplitude and PSD of the CF Direction . . . . . . . . . . . . . . . . . . 59 
B.15 $\mathrm{Vr}=5.89(\mathrm{U}=0.55 \mathrm{~m} / \mathrm{s})$, Time history of amplitude and PSD of the IL Direction . . . . . . . . . . . . . . 60

B.16 $\mathrm{Vr}=5.89(\mathrm{U}=0.55 \mathrm{~m} / \mathrm{s})$, orbital patterns $0-12 \mathrm{sec} \ldots \ldots 0$

B.17 $\mathrm{Vr}=6.27(\mathrm{U}=0.57 \mathrm{~m} / \mathrm{s})$, Spanwise shape of in-line (Black) and cross-flow (Blue) directions . . . . . . . . . . . . 61

B.18 $\mathrm{Vr}=6.27(\mathrm{U}=0.57 \mathrm{~m} / \mathrm{s})$, Time history of amplitude and PSD of the CF Direction . . . . . . . . . . . . . . 61

B.19 $\mathrm{Vr}=6.27(\mathrm{U}=0.57 \mathrm{~m} / \mathrm{s})$, Time history of amplitude and PSD of the IL Direction . . . . . . . . . . . . . . . . . . . . . . 62

B.20 $\mathrm{Vr}=6.27(\mathrm{U}=0.57 \mathrm{~m} / \mathrm{s})$, orbital patterns $0-12 \mathrm{sec} \ldots \ldots 2$

B.21 $\mathrm{Vr}=6.30(\mathrm{U}=0.58 \mathrm{~m} / \mathrm{s})$, Spanwise shape of in-line (Black) and cross-flow (Blue) directions ............ . 63

B.22 $\mathrm{Vr}=6.30(\mathrm{U}=0.58 \mathrm{~m} / \mathrm{s})$, Time history of amplitude and PSD of the CF Direction . . . . . . . . . . . . . 63

B.23 $\mathrm{Vr}=6.30(\mathrm{U}=0.58 \mathrm{~m} / \mathrm{s})$, Time history of amplitude and PSD of the IL Direction . . . . . . . . . . . . . . . . . . . . 64

B.24 $\operatorname{Vr}=6.30(\mathrm{U}=0.58 \mathrm{~m} / \mathrm{s})$, orbital patterns $0-12 \mathrm{sec} \ldots \ldots 4$

B.25 $\mathrm{Vr}=6.36(\mathrm{U}=0.61 \mathrm{~m} / \mathrm{s})$, Spanwise shape of in-line (Black) and cross-flow (Blue) directions . . . . . . . . . . 65

B.26 $\mathrm{Vr}=6.36(\mathrm{U}=0.61 \mathrm{~m} / \mathrm{s})$, Time history of amplitude and PSD of the CF Direction . . . . . . . . . . . . . 65

B.27 $\mathrm{Vr}=6.36(\mathrm{U}=0.61 \mathrm{~m} / \mathrm{s})$, Time history of amplitude and PSD of the IL Direction . . . . . . . . . . . . . . . . 66

B.28 $\mathrm{Vr}=6.36(\mathrm{U}=0.61 \mathrm{~m} / \mathrm{s})$, orbital patterns $0-12$ sec. At 6.5 seconds point tracking was lost during data analysis . . . . . . 66

B.29 $\mathrm{Vr}=5.68(\mathrm{U}=0.624 \mathrm{~m} / \mathrm{s})$, Spanwise shape of in-line (Black) and cross-flow (Blue) directions . . . . . . . . . . . 67

B.30 $\mathrm{Vr}=5.68(\mathrm{U}=0.624 \mathrm{~m} / \mathrm{s})$, Time history of amplitude and PSD of the CF Direction . . . . . . . . . . . . . . . 67 
B.31 $\mathrm{Vr}=5.68(\mathrm{U}=0.624 \mathrm{~m} / \mathrm{s})$, Time history of amplitude and PSD of the IL Direction . . . . . . . . . . . . . . . 68

B.32 $\operatorname{Vr}=5.68(\mathrm{U}=0.624 \mathrm{~m} / \mathrm{s})$, orbital patterns $0-12 \mathrm{sec} \ldots \ldots 8$

B.33 $\mathrm{Vr}=5.73(\mathrm{U}=0.647 \mathrm{~m} / \mathrm{s})$, Spanwise shape of in-line (Black) and cross-flow (Blue) directions . . . . . . . . . . . 69

B.34 $\mathrm{Vr}=5.73(\mathrm{U}=0.647 \mathrm{~m} / \mathrm{s})$, Time history of amplitude and PSD of the CF Direction . . . . . . . . . . . . . . . 69

B.35 $\mathrm{Vr}=5.73(\mathrm{U}=0.647 \mathrm{~m} / \mathrm{s})$, Time history of amplitude and PSD of the IL Direction . . . . . . . . . . . . . . . . . . . 70

B.36 $\mathrm{Vr}=5.73(\mathrm{U}=0.647 \mathrm{~m} / \mathrm{s})$, orbital patterns $0-12 \mathrm{sec} \ldots \ldots 70$

B.37 $\mathrm{Vr}=5.65(\mathrm{U}=0.66 \mathrm{~m} / \mathrm{s})$, Spanwise shape of in-line (Black) and cross-flow (Blue) directions . . . . . . . . . . . 71

B.38 $\mathrm{Vr}=5.65(\mathrm{U}=0.66 \mathrm{~m} / \mathrm{s})$, Time history of amplitude and PSD of the CF Direction . . . . . . . . . . . . . . . 71

B.39 $\mathrm{Vr}=5.65(\mathrm{U}=0.66 \mathrm{~m} / \mathrm{s})$, Time history of amplitude and PSD of the IL Direction . . . . . . . . . . . . . . . . . . 72

B.40 $\operatorname{Vr}=5.65(\mathrm{U}=0.66 \mathrm{~m} / \mathrm{s})$, orbital patterns $0-12 \mathrm{sec} \ldots . . .72$

C.1 $\operatorname{Vr}=5.89(\mathrm{U}=0.55 \mathrm{~m} / \mathrm{s})$, Spanwise shape of in-line (Black) and cross-flow (Blue) directions . . . . . . . . . . . 73

C.2 $\mathrm{Vr}=5.89(\mathrm{U}=0.55 \mathrm{~m} / \mathrm{s})$, Time history of amplitude and PSD of the CF Direction . . . . . . . . . . . . . 73

C.3 $\mathrm{Vr}=5.89(\mathrm{U}=0.55 \mathrm{~m} / \mathrm{s})$, Time history of amplitude and PSD of the IL Direction . . . . . . . . . . . . . . . . 74

C.4 $\operatorname{Vr}=5.89(\mathrm{U}=0.55 \mathrm{~m} / \mathrm{s})$, orbital patterns $0-12 \mathrm{sec} \ldots \ldots 74$

C.5 $\mathrm{Vr}=6.03(\mathrm{U}=0.57 \mathrm{~m} / \mathrm{s})$, Spanwise shape of in-line (Black) and cross-flow (Blue) directions . . . . . . . . . . . 75

C.6 $\operatorname{Vr}=6.03(\mathrm{U}=0.57 \mathrm{~m} / \mathrm{s})$, Time history of amplitude and PSD of the CF Direction . . . . . . . . . . . . . . . . . 75 
C.7 $\mathrm{Vr}=6.03(\mathrm{U}=0.57 \mathrm{~m} / \mathrm{s})$, Time history of amplitude and PSD of the IL Direction . . . . . . . . . . . . . . . 76

C.8 $\operatorname{Vr}=6.03(\mathrm{U}=0.57 \mathrm{~m} / \mathrm{s})$, orbital patterns $0-12 \mathrm{sec} \ldots \ldots$

C.9 $\operatorname{Vr}=6.00(\mathrm{U}=0.59 \mathrm{~m} / \mathrm{s})$, Spanwise shape of in-line (Black) and cross-flow (Blue) directions . . . . . . . . . . . 77

C.10 $\mathrm{Vr}=6.00(\mathrm{U}=0.59 \mathrm{~m} / \mathrm{s})$, Time history of amplitude and PSD of the CF Direction . . . . . . . . . . . . . . 77

C.11 $\operatorname{Vr}=6.00(\mathrm{U}=0.59 \mathrm{~m} / \mathrm{s})$, Time history of amplitude and PSD of the IL Direction . . . . . . . . . . . . . . . . . . . . 78

C.12 $\operatorname{Vr}=6.00(\mathrm{U}=0.59 \mathrm{~m} / \mathrm{s})$, orbital patterns $0-12 \mathrm{sec} \ldots \ldots$

C.13 $\mathrm{Vr}=6.127(\mathrm{U}=0.61 \mathrm{~m} / \mathrm{s})$, Spanwise shape of in-line (Black) and cross-flow (Blue) directions . . . . . . . . . . . 79

C.14 $\operatorname{Vr}=6.127(\mathrm{U}=0.61 \mathrm{~m} / \mathrm{s})$, Time history of amplitude and PSD of the CF Direction . . . . . . . . . . . . . . 79

C.15 $\mathrm{Vr}=6.127(\mathrm{U}=0.61 \mathrm{~m} / \mathrm{s})$, Time history of amplitude and PSD of the IL Direction . . . . . . . . . . . . . . . . . . . . . . 80

C.16 $\operatorname{Vr}=6.127(\mathrm{U}=0.61 \mathrm{~m} / \mathrm{s})$, orbital patterns $0-12 \mathrm{sec} \ldots . . . \quad 80$

C.17 $\mathrm{Vr}=6.13(\mathrm{U}=0.63 \mathrm{~m} / \mathrm{s})$, Spanwise shape of in-line (Black) and cross-flow (Blue) directions . . . . . . . . . . . 81

C.18 $\mathrm{Vr}=6.13(\mathrm{U}=0.63 \mathrm{~m} / \mathrm{s})$, Time history of amplitude and PSD of the CF Direction . . . . . . . . . . . . . . . 81

C.19 $\mathrm{Vr}=6.13(\mathrm{U}=0.63 \mathrm{~m} / \mathrm{s})$, Time history of amplitude and PSD of the IL Direction . . . . . . . . . . . . . . . . . 82

C.20 $\operatorname{Vr}=6.13(\mathrm{U}=0.63 \mathrm{~m} / \mathrm{s})$, orbital patterns $0-12 \mathrm{sec} \ldots \ldots 2$

C.21 $\mathrm{Vr}=6.35(\mathrm{U}=0.65 \mathrm{~m} / \mathrm{s})$, Spanwise shape of in-line (Black) and cross-flow (Blue) directions . . . . . . . . . . 83

C.22 $\mathrm{Vr}=6.35(\mathrm{U}=0.65 \mathrm{~m} / \mathrm{s})$, Time history of amplitude and PSD of the CF Direction . . . . . . . . . . . . . . . . 83 
C.23 $\mathrm{Vr}=6.35(\mathrm{U}=0.65 \mathrm{~m} / \mathrm{s})$, Time history of amplitude and PSD of the IL Direction . . . . . . . . . . . . . . . . 84

C.24 $\operatorname{Vr}=6.35(\mathrm{U}=0.65 \mathrm{~m} / \mathrm{s})$, orbital patterns $0-12 \mathrm{sec} \ldots . . .84$

C.25 $\mathrm{Vr}=6.46(\mathrm{U}=0.66 \mathrm{~m} / \mathrm{s})$, Spanwise shape of in-line (Black) and cross-flow (Blue) directions ............. . 85

C.26 $\mathrm{Vr}=6.46(\mathrm{U}=0.66 \mathrm{~m} / \mathrm{s})$, Time history of amplitude and PSD of the CF Direction . . . . . . . . . . . . . . . 85

C.27 $\mathrm{Vr}=6.46(\mathrm{U}=0.66 \mathrm{~m} / \mathrm{s})$, Time history of amplitude and PSD of the IL Direction . . . . . . . . . . . . . . . . . . . 86

C.28 $\operatorname{Vr}=6.46(\mathrm{U}=0.66 \mathrm{~m} / \mathrm{s})$, orbital patterns $0-12 \mathrm{sec} \ldots \ldots 6$

C.29 $\mathrm{Vr}=5.90(\mathrm{U}=0.69 \mathrm{~m} / \mathrm{s})$, Spanwise shape of in-line (Black) and cross-flow (Blue) directions . . . . . . . . . . 87

C.30 $\operatorname{Vr}=5.90(\mathrm{U}=0.69 \mathrm{~m} / \mathrm{s})$, Time history of amplitude and PSD of the CF Direction . . . . . . . . . . . . . . . 87

C.31 $\mathrm{Vr}=5.90(\mathrm{U}=0.69 \mathrm{~m} / \mathrm{s})$, Time history of amplitude and PSD of the IL Direction . . . . . . . . . . . . . . . . . . 88

C.32 $\mathrm{Vr}=5.90(\mathrm{U}=0.69 \mathrm{~m} / \mathrm{s})$, orbital patterns $0-12 \mathrm{sec} \ldots \ldots . . \quad 88$

C.33 $\mathrm{Vr}=5.13(\mathrm{U}=0.70 \mathrm{~m} / \mathrm{s})$, Spanwise shape of in-line (Black) and cross-flow (Blue) directions . . . . . . . . . . . . 89

C.34 $\mathrm{Vr}=5.13(\mathrm{U}=0.70 \mathrm{~m} / \mathrm{s})$, Time history of amplitude and PSD of the CF Direction . . . . . . . . . . . . . . 89

C.35 $\mathrm{Vr}=5.13(\mathrm{U}=0.70 \mathrm{~m} / \mathrm{s})$, Time history of amplitude and PSD of the IL Direction . . . . . . . . . . . . . . . . . 90

C.36 $\operatorname{Vr}=5.13(\mathrm{U}=0.70 \mathrm{~m} / \mathrm{s})$, orbital patterns $0-12 \mathrm{sec} \ldots \ldots$

C.37 $3 \mathrm{Vr}=5.10(\mathrm{U}=0.72 \mathrm{~m} / \mathrm{s})$, Spanwise shape of in-line (Black) and cross-flow (Blue) directions . . . . . . . . . . . . 91

C.38 $\mathrm{Vr}=5.10(\mathrm{U}=0.72 \mathrm{~m} / \mathrm{s})$, Time history of amplitude and PSD of the CF Direction . . . . . . . . . . . . . . . . . . 91 
C.39 $\mathrm{Vr}=5.10(\mathrm{U}=0.72 \mathrm{~m} / \mathrm{s})$, Time history of amplitude and PSD of the IL Direction . . . . . . . . . . . . . . . . . 92

C.40 $\operatorname{Vr}=5.10(\mathrm{U}=0.72 \mathrm{~m} / \mathrm{s})$, orbital patterns $0-12 \mathrm{sec} \ldots . . .92$

C.41 $\mathrm{Vr}=5.08(\mathrm{U}=0.73 \mathrm{~m} / \mathrm{s})$, Spanwise shape of in-line (Black) and cross-flow (Blue) directions . . . . . . . . . . . . 93

C.42 $\mathrm{Vr}=5.08(\mathrm{U}=0.73 \mathrm{~m} / \mathrm{s})$, Time history of amplitude and PSD of the CF Direction . . . . . . . . . . . . . . . . . 93

C.43 $\mathrm{Vr}=5.08(\mathrm{U}=0.73 \mathrm{~m} / \mathrm{s})$, Time history of amplitude and PSD of the IL Direction . . . . . . . . . . . . . . . . . . . . . . . . . 94

C.44 $\operatorname{Vr}=5.08(\mathrm{U}=0.73 \mathrm{~m} / \mathrm{s})$, orbital patterns $0-12 \mathrm{sec} \ldots \ldots . . .94$

C.45 $\mathrm{Vr}=5.09(\mathrm{U}=0.75 \mathrm{~m} / \mathrm{s})$, Spanwise shape of in-line (Black) and cross-flow (Blue) directions . . . . . . . . . . . . 95

C.46 $\mathrm{Vr}=5.09(\mathrm{U}=0.75 \mathrm{~m} / \mathrm{s})$, Time history of amplitude and PSD of the CF Direction . . . . . . . . . . . . . . 95

C.47 $\mathrm{Vr}=5.09(\mathrm{U}=0.75 \mathrm{~m} / \mathrm{s})$, Time history of amplitude and PSD of the IL Direction . . . . . . . . . . . . . . . . 96

C.48 $\operatorname{Vr}=5.09(\mathrm{U}=0.75 \mathrm{~m} / \mathrm{s})$, orbital patterns $0-12 \mathrm{sec} \ldots \ldots 6$

C.49 $\mathrm{Vr}=5.14(\mathrm{U}=0.77 \mathrm{~m} / \mathrm{s})$, Spanwise shape of in-line (Black) and cross-flow (Blue) directions . . . . . . . . . . . 97

C.50 $\mathrm{Vr}=5.14(\mathrm{U}=0.77 \mathrm{~m} / \mathrm{s})$, Time history of amplitude and PSD of the CF Direction . . . . . . . . . . . . . . . 97

C.51 $\mathrm{Vr}=5.14(\mathrm{U}=0.77 \mathrm{~m} / \mathrm{s})$, Time history of amplitude and PSD of the IL Direction . . . . . . . . . . . . . . . . . . 98

C.52 $\operatorname{Vr}=5.14(\mathrm{U}=0.77 \mathrm{~m} / \mathrm{s})$, orbital patterns $0-12 \mathrm{sec} \ldots \ldots$

D.1 $\mathrm{Vr}=5.11(\mathrm{U}=0.59 \mathrm{~m} / \mathrm{s})$ Spanwise shape of in-line (Black) and cross-flow (Blue) directions . . . . . . . . . . . . . 99

D.2 $\mathrm{Vr}=5.11(\mathrm{U}=0.59 \mathrm{~m} / \mathrm{s})$ Time history of amplitude and PSD of the CF Direction . . . . . . . . . . . . . . . . . . . . . . 99 
D.3 $\mathrm{Vr}=5.11(\mathrm{U}=0.59 \mathrm{~m} / \mathrm{s})$ Time history of amplitude and PSD of the IL Direction . . . . . . . . . . . . . . . . 100

D.4 $\mathrm{Vr}=5.11(\mathrm{U}=0.59 \mathrm{~m} / \mathrm{s})$ orbital patterns $0-12 \mathrm{sec} \ldots \ldots$. . . 100

D.5 $\mathrm{Vr}=5.25(\mathrm{U}=0.60 \mathrm{~m} / \mathrm{s})$ Spanwise shape of in-line (Black) and cross-flow (Blue) directions . . . . . . . . . . . . . 101

D.6 $\mathrm{Vr}=5.25(\mathrm{U}=0.60 \mathrm{~m} / \mathrm{s})$ Time history of amplitude and PSD of the CF Direction . . . . . . . . . . . . . . . . . 101

D.7 $\mathrm{Vr}=5.25(\mathrm{U}=0.60 \mathrm{~m} / \mathrm{s})$ Time history of amplitude and PSD of the IL Direction . . . . . . . . . . . . . . . . . . . . . . . 102

D.8 $\mathrm{Vr}=5.25(\mathrm{U}=0.60 \mathrm{~m} / \mathrm{s})$ orbital patterns $0-12 \mathrm{sec} \ldots . . . .102$

D.9 $\mathrm{Vr}=5.35(\mathrm{U}=0.62 \mathrm{~m} / \mathrm{s})$ Spanwise shape of in-line (Black) and cross-flow (Blue) directions . . . . . . . . . . . . . 103

D.10 $\mathrm{Vr}=5.35(\mathrm{U}=0.62 \mathrm{~m} / \mathrm{s})$ Time history of amplitude and PSD of the CF Direction . . . . . . . . . . . . . . . . 103

D.11 $\mathrm{Vr}=5.35(\mathrm{U}=0.62 \mathrm{~m} / \mathrm{s})$ Time history of amplitude and PSD of the IL Direction . . . . . . . . . . . . . . . . . . . . . . 104

D.12 $\operatorname{Vr}=5.35(\mathrm{U}=0.62 \mathrm{~m} / \mathrm{s})$ orbital patterns $0-12 \mathrm{sec} . . . . . .104$

D.13 $\mathrm{Vr}=5.41(\mathrm{U}=0.65 \mathrm{~m} / \mathrm{s})$ Spanwise shape of in-line (Black) and cross-flow (Blue) directions . . . . . . . . . . . 105

D.14 $\mathrm{Vr}=5.41(\mathrm{U}=0.65 \mathrm{~m} / \mathrm{s})$ Time history of amplitude and PSD of the CF Direction . . . . . . . . . . . . . . . 105

D.15 $\mathrm{Vr}=5.41(\mathrm{U}=0.65 \mathrm{~m} / \mathrm{s})$ Time history of amplitude and PSD of the IL Direction . . . . . . . . . . . . . . . 106

D.16 $\mathrm{Vr}=5.41(\mathrm{U}=0.65 \mathrm{~m} / \mathrm{s})$ orbital patterns $0-12 \mathrm{sec} \ldots \ldots 106$

D.17 $\mathrm{Vr}=5.35(\mathrm{U}=0.66 \mathrm{~m} / \mathrm{s})$ Spanwise shape of in-line (Black) and cross-flow (Blue) directions . . . . . . . . . . . 107

D.18 $\mathrm{Vr}=5.35(\mathrm{U}=0.66 \mathrm{~m} / \mathrm{s})$ Time history of amplitude and PSD of the CF Direction . . . . . . . . . . . . . . . . 107 
D.19 $\mathrm{Vr}=5.35(\mathrm{U}=0.66 \mathrm{~m} / \mathrm{s})$ Time history of amplitude and PSD of the IL Direction . . . . . . . . . . . . . . . . 108

D.20 $\mathrm{Vr}=5.35(\mathrm{U}=0.66 \mathrm{~m} / \mathrm{s})$ orbital patterns $0-12 \mathrm{sec} \ldots \ldots . . . .108$

D.21 $\mathrm{Vr}=5.40(\mathrm{U}=0.69 \mathrm{~m} / \mathrm{s})$ Spanwise shape of in-line (Black) and cross-flow (Blue) directions . . . . . . . . . . . . 109

D.22 $\mathrm{Vr}=5.40(\mathrm{U}=0.69 \mathrm{~m} / \mathrm{s})$ Time history of amplitude and PSD of the CF Direction . . . . . . . . . . . . . . . . . . 109

D.23 $\mathrm{Vr}=5.40(\mathrm{U}=0.69 \mathrm{~m} / \mathrm{s})$ Time history of amplitude and PSD of the IL Direction . . . . . . . . . . . . . . . . . . . . . . 110

D.24 $\mathrm{Vr}=5.40(\mathrm{U}=0.69 \mathrm{~m} / \mathrm{s})$ orbital patterns $0-12 \mathrm{sec} . . . . .110$

D.25 $\mathrm{Vr}=5.13(\mathrm{U}=0.70 \mathrm{~m} / \mathrm{s})$ Spanwise shape of in-line (Black) and cross-flow (Blue) directions . . . . . . . . . . . . 111

D.26 $\mathrm{Vr}=5.13(\mathrm{U}=0.70 \mathrm{~m} / \mathrm{s})$ Time history of amplitude and PSD of the CF Direction . . . . . . . . . . . . . . . . . 111

D.27 $\mathrm{Vr}=5.13(\mathrm{U}=0.70 \mathrm{~m} / \mathrm{s})$ Time history of amplitude and PSD of the IL Direction . . . . . . . . . . . . . . . . . . . . . 112

D.28 $\operatorname{Vr}=5.13(\mathrm{U}=0.70 \mathrm{~m} / \mathrm{s})$ orbital patterns $0-12 \mathrm{sec} \ldots . . . .112$

D.29 $\mathrm{Vr}=5.36(\mathrm{U}=0.72 \mathrm{~m} / \mathrm{s})$ Spanwise shape of in-line (Black) and cross-flow (Blue) directions . . . . . . . . . . . 113

D.30 $\mathrm{Vr}=5.36(\mathrm{U}=0.72 \mathrm{~m} / \mathrm{s})$ Time history of amplitude and PSD of the CF Direction . . . . . . . . . . . . . . . . . 113

D.31 $\mathrm{Vr}=5.36(\mathrm{U}=0.72 \mathrm{~m} / \mathrm{s})$ Time history of amplitude and PSD of the IL Direction . . . . . . . . . . . . . . . . . . 114

D.32 $\mathrm{Vr}=5.36(\mathrm{U}=0.72 \mathrm{~m} / \mathrm{s})$ orbital patterns $0-12 \mathrm{sec} . \ldots . . .114$

D.33 $\mathrm{Vr}=5.30(\mathrm{U}=0.73 \mathrm{~m} / \mathrm{s})$ Spanwise shape of in-line (Black) and cross-flow (Blue) directions . . . . . . . . . . . 115

D.34 $\mathrm{Vr}=5.30(\mathrm{U}=0.73 \mathrm{~m} / \mathrm{s})$ Time history of amplitude and PSD of the CF Direction . . . . . . . . . . . . . . . . 115 
Figure

D.35 $\mathrm{Vr}=5.30(\mathrm{U}=0.73 \mathrm{~m} / \mathrm{s})$ Time history of amplitude and PSD of the IL Direction . . . . . . . . . . . . . . 116

D.36 $\mathrm{Vr}=5.30(\mathrm{U}=0.73 \mathrm{~m} / \mathrm{s})$ orbital patterns $0-12 \mathrm{sec} \ldots \ldots 116$ 


\section{CHAPTER 1}

\section{Introduction}

Analyzing the phenomena of vortex-induced vibration (VIV) requires knowledge about fluid dynamics, structural vibrations, and complex techniques for data analysis and numerical simulation. Many offshore structures encounter VIV, which need to be accounted for in their design. VIV results from vortex shedding in the wake of bluff bodies and can occur in a variety of structures which include drilling risers, spar buoys mooring lines, and underwater cables and piping. Due to these fluid-structure interactions, the design of deep water systems is a challenging engineering problem and the understanding of VIV, and its suppression are very active areas of research.

\subsubsection{Research Motivation}

The primary goal of this research is the examine vortex-induced vibrations (VIV) in the context of ocean structures. The discovery of oil and natural gas fields in the some of the deepest parts of the ocean have prompted the need for engineering solutions to retrieve natural resources at extreme depths. Extremely long risers and cables operating in these extreme depths will be plagued with vibrational problems due to very high mode numbers, becoming susceptible to excitations over a broad range of frequencies (Dahl, 2008) .

Previous studies have shown that VIV of marine structures can cause large amplitude motions. These large amplitude motions, or vibrations, can lead to fatigue damage or shutdown of offshore operations due to increased drag loads as suggested by Vandiver and Jong (1987). The large amplitude motions observed by Dahl (2006, 2008), Feng (1968), Jauvtis and Williamshon (2004), King (1948, 1973), indicate that an increased drag coefficient alters the static configuration, 
thereby increasing the static loading (Every et al 1981, Yoeger et al 1991) and dynamic loads at the forcing frequency, reducing fatigue life (Hover et al 1996). The findings observed by Vandiver and Jong (1987) show large magnitude motions of a riser $75 \mathrm{ft}(22.86 \mathrm{~m})$ long with a outside diameter of $1.25 \mathrm{in}(3.175 \mathrm{~cm})$, and an inner diameter of 1 in $(2.54 \mathrm{~cm})$ on the order of one diameter and half-diameter in the in-line direction and cross-flow directions respectively. Similar results have been found in experiments conducted when using elastically mounted cylinders as in Jauvtis and Williamson (2004) and fixed vibration experiments performed by Jeon and Gharib (2001).

These oscillations can be irregular and random on long slender, flexible structures but under certain conditions, regular patterns such as figure-eights have been observed (Vandiver 1987, Dahl 2006, Dahl 2008). The circumstances under which irregular of regular oscillations occur are not clearly understood. However, very few studies have examined the relationship, if any, of a modal, or time spatial ratio on such patterns of oscillation. Understanding the role in which the spatial shapes (mode shape) of the riser effects the interaction within the wake will improve the ability to predict fatigue life in those structures as well as answer the following:

1. How does mode shape affect VIV?

2. Does the interaction of different modes in in-line and cross flow directions lead to irregular or regular variations?

3. How is the cylinder wake affected by the modal excitation of the cylinder?

This thesis begins with the study of vibrations of a flexible cylinder mounted in a uniform flow. The ratio of natural frequencies in the in-line and cross-flow directions can be tuned independently, allowing for adjustments of the mode or spatial ratio. The mass ratio is designed to be low, comparable to the mass ratio 
of ocean structures. An analysis of cylinder oscillations with three unique mode ratios, each with the same frequency ratio will be able to provide insight to the questions listed.

\subsubsection{Chapter Preview}

Chapter 2 discusses much of the background information on vortex shedding, vortex induced vibrations, and nomenclature used throughout the thesis. It also provides a brief summary of literature regarding vortex-induced vibrations flexibly mounted with one or two-degrees of freedom, and flexible cylinders.

Chapter 3 explains the flexible cylinder experiments, a description of the methods is given, along with the results of the experiment. A discussion of the results emphasizes the effects of mode shape on cylinder response.

Chapter 4 is the conclusion of this thesis and contributions of this thesis to engineering are outlined along with recommendations for future work. 


\section{CHAPTER 2}

\section{Vortex Induced Vibrations - Fluid Properties}

In this chapter, the basic concept of vortex shedding and vortex induced vibrations are explained and previous research is examined to illustrate the relevant parameters associated with vortex-induced vibrations. Past simplifications in analyzing vortex-induced vibrations are shown and the relevant non-dimensional parameters for properly modeling and scaling the forces associated with vortex induced vibrations are defined. The emphasis is given to the last section of this chapter where the problem of mode ratio variation is introduced with an explanation of the work performed by Dahl (2008) .

\subsection{Vortex Shedding}

At very low Reynolds numbers $\left(R_{e}<5\right)$ the streamlines of a two-dimensional flow will remain unseparated with stagnation points both fore and aft of the cylinder. As the Reynolds numbers increase $\left(5<R_{e}<40\right)$, a pair of fixed vortices are formed in the wake behind the cylinder, yet remain attached. Beyond $R_{e}=40$, the wake becomes unstable and small perturbations in the flow or on the surface of the cylinder will cause vortices to break of from the cylinder and shed periodically, also known as the formation of the Kármán-Bénard vortex street. The shedding of a vortex occurs when the shear layer of the next upcoming vortex interacts with the opposite side shear layer, canceling its vorticity because of a its different sign. As this process is repeated on each side of the cylinder, a vortex street is generated

in the wake of the cylinder (Figure 1). The frequency of shedding can be expressed non-dimensionally according to the Strouhal number,

$$
S_{t}=\frac{f_{s} D}{U}
$$


where $S_{t}$ is the Stouhal number, $f_{s}$ is the shedding frequency, $D$, the diameter, and flow velocity, $U$. Numerous studies (Newman, 1977; Norberg, 2003) have shown that a $S_{t}$ value of approximately 0.2 can be determined when the $R_{e}$ is in the subcritical regime. When vortex shedding occurs in the downstream wake of an elastically mounted of flexible stationary circular cylinder, alternating fluid forces are generated on the cylinder in which vortex-induced vibrations (VIV), or cylinder motions can occur. The phenomena of vortex shedding is shown in Figure 1.

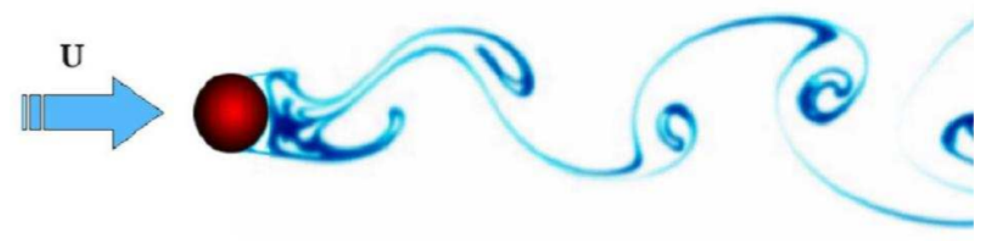

Figure 1: Image modified by Dahl from picture by S. Taneda in Van Dyke

\subsubsection{Vortex-Induced Vibrations}

When a cylinder is flexible or elastically mounted, the natural frequency of the cylinder can be excited by vortex shedding. When the frequency of vortex shed$\operatorname{ding}, f_{s}$ is near the natural frequency $f_{n}$ of the cylinder, large amplitude motions can be observed. These motions are called vortex-induced vibrations (VIV) and are caused by the interaction of the cylinder structure with vortices shed in the cylinder wake. VIV is a subset of fluid mechanics instabilities called flow-induced vibrations. Unlike unstable oscillations where geometrical asymmetry can cause extreme loading that can cause failure when exceeding yield strength properties, vortex-induced vibrations are typically moderate in amplitude and do not result in immediate failure. Vortex-induced vibrations result in material fatigue over a large number of oscillation cycles, or failure by fatigue. Ocean structures are designed to be built in excess of 20 years (Det Norske Veritas 2011), and the fatigue life of 
these structures becomes essential when defining the design criteria.

Vortex induced vibrations (VIV) have become an important engineering problem often associated with long and slender structures that are characterized by a large number of natural frequencies,. From an ocean engineering perspective, the ultimate goals of studies in vortex-induced vibrations are to be able to predict, control, or suppress vibrations that cause fatigue damage in offshore structures, risers and mooring lines. In addition to offshore structures, towed equipment, such as SONAR, and subsea piping can be susceptible to vibrations.

All of these applications share in common a bluff body structure, defined as having a cross sectional shape with a span-to-chord ratio near one (high aspect ratio). These vibrations can be very complex due to the high number of structural natural modes and sheared ocean currents. A better understanding of vortexinduced vibrations can be provided by making a couple of assumptions.

One simplification for studying the flow around the length of a long body, is to examine the flow around a small section. A small section will appear as an elastically mounted cylinder and the sheared current will appear as a twodimensional free stream of fluid with a constant velocity. This assumption allows one to understand the basic mechanisms that lead to the vibrations of the body.

When vortices shed in the wake of the cylinder, a single vortex (or group of vortices) is shed on one side of the cylinder per cycle repeating on the other side. This implies that the forcing frequency in the lift direction occurs at the frequency of vortex shedding. In the drag direction however; all vortices are shed downstream, therefore the drag force associated with vortex shedding must occur at twice the frequency of the lift force. When the frequency of vortex shedding nears the natural frequency of an elastically mounted cylinder, response motions show a relatively large motion in the transverse (perpendicular to the fluid flow) 
direction close to one cylinder diameter while the in-line (in the direction of the fluid flow) motions are typically less the half of the diameter. For this reason, many studies simplify the problem by considering only transverse or in-line vibrations of the structure. Only recently have studies begun to consider the effects of in-line motions. Several reviews of VIV studies [Sarpkaya (1979, 2004); Bearman (1984, 2011); Parkinson (1974); Williamson and Govardhan (2004)] highlight a number of observations and experiments that have been performed over the past few decades.

\subsubsection{Relevant Parameters for Analysis of Vortex Shedding and VIV}

Below are the relevant non-dimensional parameters associated with vortexinduced vibrations.

The true reduced velocity, $V_{r}$, is similar to the inverse Strouhal number where the frequency of cylinder motion is used to normalize the fluid velocity, therefore making a comparison of the free-stream velocity with respect to the cylinders crossflow velocity or oscillation velocity. This term needs to be matched in order to compare the free vibration of a cylinder with forced vibrations.

$$
V_{r}=\frac{U}{f_{e} D}
$$

where $f_{e}$ is the excitation frequency, or dominating vibration frequency. In some cases however; the preference is to use the nominal reduced velocity $V_{r n}$ since the natural frequency of the structure will remain constant during experiments.

$$
V_{n r}=\frac{U}{f_{n y} D}
$$

The amplitude ratio $\left(\frac{A_{y}}{D}\right)$ and aspect ratio $\left(\frac{L}{D}\right)$ is a measured function of either the true or nominal reduced velocity.

In air, the mass ratio defined as the cyclical mass over the displaced fluid mass 


$$
m^{*}=\frac{m}{\rho \pi\left(\frac{D^{2} S}{4}\right)}
$$

is typically very large since the density of the fluid is very small. In water, the fluid density will be on the same order of magnitue as the density of the cylinder.

Reynolds number characterizes the flow comparing inertial and viscous forces and is defined based on the cylinder diameter. The recent studies of Govardhan and Williamson [Dahl thesis 20] have shown that the amplitudes and forces associated with vortex induced vibrations have a dependence on Reynolds number as well.

\subsection{Literature Review}

\subsubsection{Rigid Cylinders: One Degree of Freedom (Transverse Only)}

In the one degree of freedom vortex induced vibration problem, an elastically mounted circular cylinder is situated in a free stream of fluid shown in Figure 2

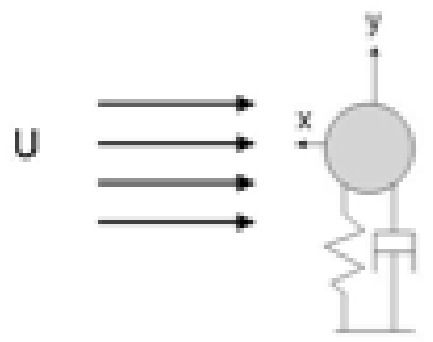

Figure 2: One Dimension Spring-Pot System Dahl, 2008

When undergoing vortex shedding, or a forced vibration, the fluid forces acting upon the body are influenced by the relative motions of the cylinder with respect to the vortices in the wake. As the wake changes as a function of the body motion, the body is forced differently, resulting in a change to the wake. A dynamic equilibrium is reached through this feedback mechanism (wake driving the body and vice versa). The formation of vortices is caused by the instabilities in the wake behind the cylinder. As a result, vortex shedding is triggered by the slightest flow 
perturbations and excitation of the structure is unavoidable. An experiment that reviewed the behavior of a flexibly mounted cylinder restricted to move only in the cross flow direction (to the flow) was carried out by Feng (1968). Feng found that the frequency of vibration was very close to the natural frequency of the system in air ( $m^{*}$ was very large) and that the vortex shedding frequency followed the Strouhal relationship until it began to approach the frequency of oscillations, near the natural frequency of the system. The instability of the flow begins to occur at the Strouhal frequency. Once the structure moves, the frequency of vortex shedding will become entrained to the natural frequency of structure oscillation, within a band around the Strouhal frequency. The frequency band will increase with an increasing amplitude of motion, and this property of VIV is called the wakecapture phenomenon (Dahl 2008). This ability of the wake to follow the oscillation frequency of the structure results in substantial alterations of added mass forces, which can drive the effective natural frequency of the system to different values than the nominal natural frequency. A dynamic equilibrium is reached, dictated by frequency resonance and flow-structure energy balance. This phenomenon is called lock-in.

Under this phenomena known as lock-in (sometimes referred to as resonance or synchronization), higher amplitude responses are observed. During lock-in, vortex shedding is not controlled by the Strouhal number, but instead by the cylinder motion. Extensive research has been performed concerning the lock-in phenomena [Blevins 1990 ; Sumer and Fredsoe 1997]. In a later paper, Khalak and Williamson (1999) found that bodies can oscillate at large amplitudes when not close to the natural frequency, and suggested a more suitable definition of lock-in as the matching of the frequency of the periodic wake vortex mode with the body oscillation. A more generalized definition was described by Sarpkaya (1995) as 


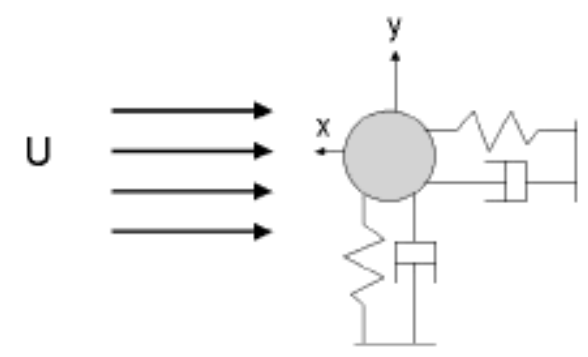

Figure 3: Two Dimensional Spring-Pot System

when the frequency of motion must match the shedding frequency.

\subsubsection{Rigid Cylinders: One Degree of Freedom (In-line Only)}

Various studies [King et al, 1974; Vandiver 1987] have found that if a cylinder is mounted in the in-line direction, the oscillating drag produced by vortex shedding may induce in-line vibrations. In general these vibrations will occur at roughly twice the frequency for transverse vibration. These vibrations pose numerous problems with underwater pipelines because larger vibrations will typically occur at very low reduced velocities.

\subsubsection{Rigid Cylinders: Two Degrees of Freedom}

In the case of motion in both in-line and cross flow directions, two linear systems are needed to describe the displacements of a cylinder. In the two degree of freedom (DOF) vortex induced vibration problem, an elastically mounted circular cylinder is situated in a free stream of fluid as shown in Figure 3.

When setting the natural frequency of the cylinder in the in-line direction equal to the cross flow directions, Jauvtis and Williamson (2004) found that there were much larger cross-flow motions and large third harmonic forcing terms when 2-DOF motions were allowed. However; the freedom to oscillate simultaneously inline and transverse to the flow does not modify lock-in. Jeon and Gharib (2001) 
used Digital Particle Image Velocimetry (DPIV) to identify changes in vortex patterns when forced cross-flow motions where introduced. When only a transverse vibrations were observed, pairs of vortices were observed; however, the wake reverts to shedding single vortices once in-line motions were added.

\subsubsection{Discussion on Orbital Patterns}

The free vibrations of a circular cylinder in two degrees of freedom become significantly more complex than those witnessed in one-degree of freedom. The additional in-line motions change the wake formation and the dynamic interaction between the body and shedding vortices. In a 2-DOF system, there are four parameters governing the spatial motion of the cylinder, the transverse amplitude, in-line amplitude, phase between the two motions, and reduced velocity. The most useful way to display all of these parameters is to show the cylinder orbital. The orbitals show the motion of the cylinder in a fixed coordinate system, such that figure-eight or crescent shapes appear as seen in Figure 4.

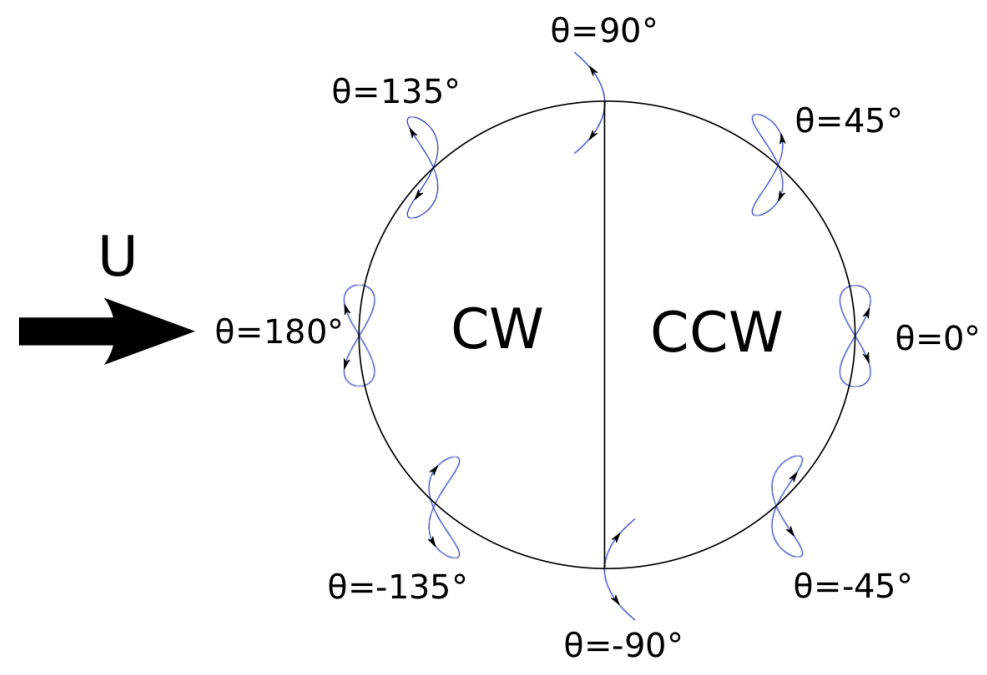

Figure 4: Cylinder orbital trajectories

The definition of $\theta$ can be found using the equations of motion for $x$ and $y$, 


$$
\begin{gathered}
y=A_{y} \sin (\omega t) \\
x=A_{x} \sin (2 \omega t+\theta)
\end{gathered}
$$

Sarpkaya (1995) noted that a flexible structure can have multiple natural frequencies in both the in-line and cross flow directions. To simplify the problem, Dahl et al (2007) performed a series of experiments using six different in-line natural frequency to transverse natural frequency ratios between 1 and 1.9. This experiment aimed to model a long slender structure which may have different modes and different natural frequencies excited separately, investigating how different ratios of natural frequencies would affect the motion and forces exerted on the structure. The variation of the natural frequencies in the in-line direction showed significant effects in the cylinder displacement. Dahl (2006) showed that for natural frequency ratios near 2, the effective natural frequency ratio adjusted for the effective added mass of the system will almost always take a value of 2 . The same phenomenon holds for supercritical Reynolds numbers (Dahl et al, 2010). The importance of the observations made by Dahl et al, show that the cylinder is subject to large suction forces caused by shedding vortices resulting in high frequency lift forces. Any trajectory other than the figure eight pattern as shown in Figure 5, would suggest weakening forces, meaning that small in-line motions may have a large impact on transverse oscillations. Overall the lift forced produced by VIV are not always dominated by the fundamental frequency near the Strouhal frequency, but can have significantly higher frequency components, and perhaps the shape of the mode(s) being excited in the in-line and transverse directions. 


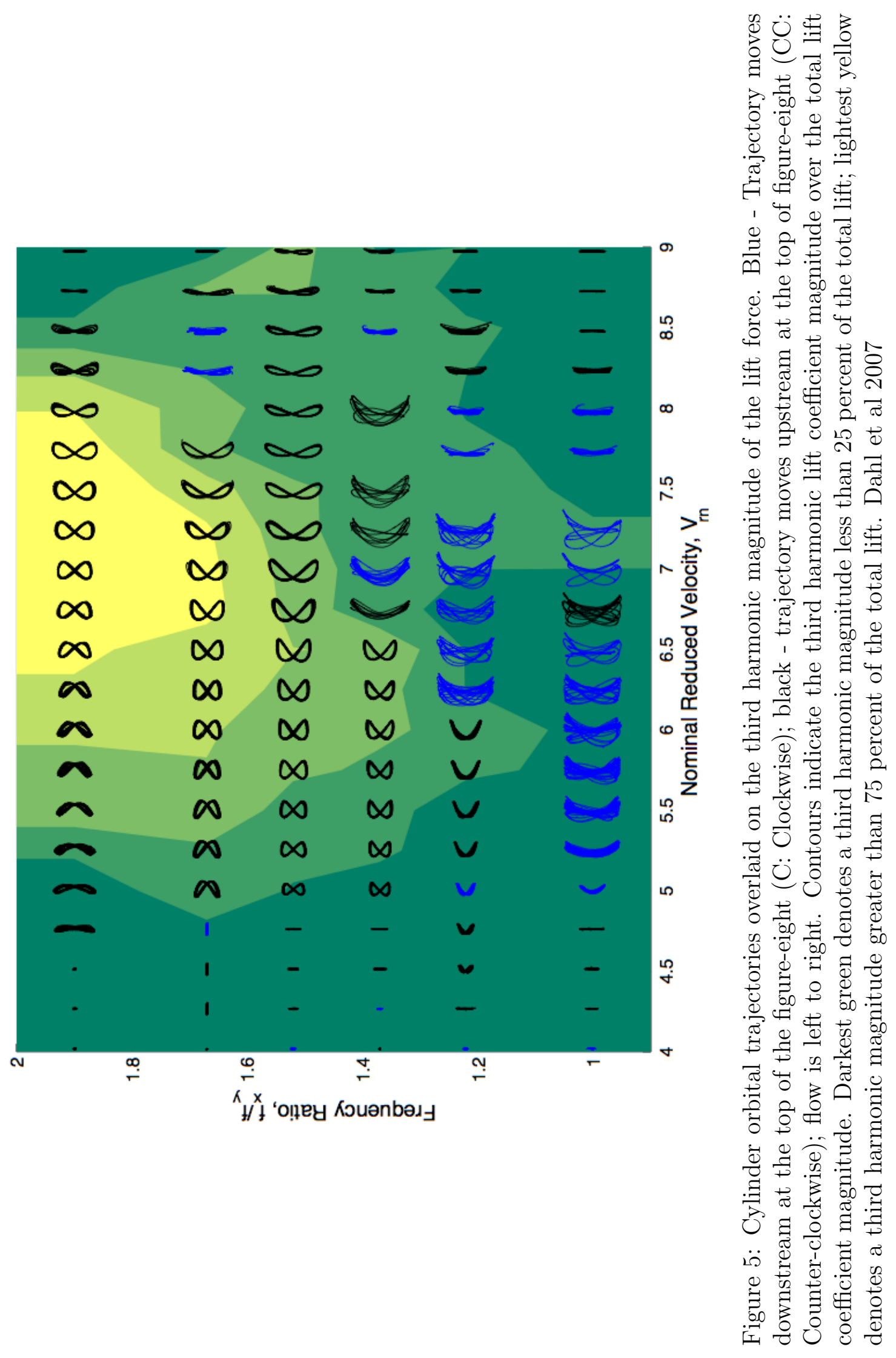


Figure 5 shows that for a frequency ratio of 1.0, figure-eight oscillations were observed and at higher frequency ratios crescent-shaped oscillation patterns become dominant. One distinct characteristic of the cylinder motions is that downstream crescent shaped motions (corresponding to $\theta \approx 90$ degrees) are typical for the largest transverse amplitudes. Similarly, third harmonic forces are more dominant if the motion is a figure eight shape with the cylinder moving upstream at the extreme transverse motions (corresponding to $\theta \approx 0$ degrees). When the orbital motions were regular, the observed excitation frequency of the in-line direction was found to always be twice the frequency of motion in the transverse direction. This is clearly shown at higher nominal frequency ratios.

Kang et al (2013) expanded on the experiments performed by Dahl, 2006 to show the response of cylinders with natural frequency ratios lower than 1 , showing that the cylinder may exhibit a multi-frequency response with slanted circle, teardrop, or egg shaped orbital motions.

While this thesis does not directly include force measurements, it may be be possible to infer new information when studying orbital motions of the cylinder, the path direction and orbit shape are important indicators of the higher harmonic forcing.

\subsubsection{Flexible Circular Cylinders}

The study of vortex induced vibrations of flexible structures has become a major focus of the offshore industry with the aim of improving the design of offshore structures and risers. High current profiles (Richardson et al. 2004) found in depth up to 3000 meters have increased the need for better understanding of this phenomena. Numerous studies have focused on this problem, but there has been an increasing difficulty to directly relate the results from one physical situation to another. Under normal conditions, vortex shedding will excite several modes 
of vibration in the structure making the study of VIV on flexible cylinders much more complex because they are characterized by multi-modal and multi-frequency vibrations. The structural characteristics of the cylinder then become important factors that must be taken into account during experimental procedures.

When vortices are shed downstream of the structure, vortex shedding produces a forcing frequency in the in-line direction to that of the cross-flow direction. Experiments on flexible structures including field experiments (Vandiver et al, 2005, Vandiver and Jong, 1987), controlled laboratory experiments (Passano et al, 2010), and full scale observation of marine risers (Trim et al, 2005) have demonstrated that for long, flexible bodies, it is possible to excite different modes separately in the cross-flow and in-line directions.

Of the many flexible cylinder experiments that have been conducted in the past, Vandiver's (1983) reports on long flexible cylinders subjected to uniform flow at Castine, Maine have become essential in understanding the physics behind VIV on flexible structures. Under lock-in conditions, a ratio of 2 between the in-line and the cross flow frequency was observed. The motions in the cross flow direction were found to be much higher than those discovered in-line. King (1977) reported on field experiments subjected to vertical sheared flow in which they found that infinite string behavior with dampening along the length of the structure. Modarres-Sadeghi et al (2011) found that the motions of long slender structures undergoing VIV are complex, potentially consisting of traveling waves, large amplitude standing waves and chaotic or unrepeatable motions. In order to visualize such cylinder motions we must first consider a flexible cylinder which has multiple natural frequencies meaning that at any given point along the length will have an in-line to transverse natural frequency ratio of $2: 1$ due to the factor $n$ modal relationship of natural frequencies. 


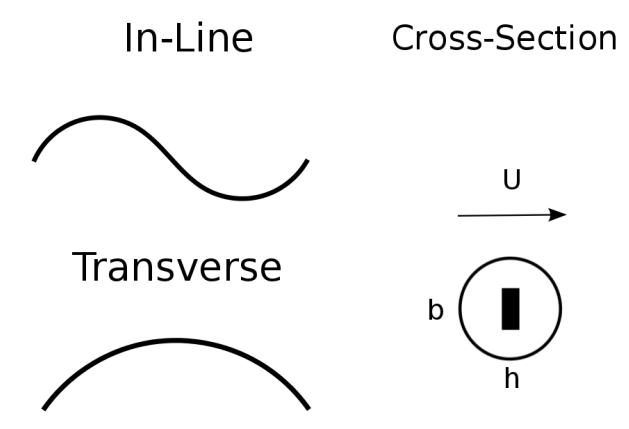

Figure 6: 1-2 In-line and Transverse Modes

A flexible cylinder under tension will act much like a string under tension exhibiting a natural frequency according to

$$
f_{\text {string }}=\frac{n}{2 L} \sqrt{\frac{T}{M}}
$$

where $f_{\text {string }}$ is the fundamental natural frequency, $L$ is the length of the string, $T$ is the tension in the string, and $M$ is the mass per length of the string. When vortices are shed downstream of the structure, vortex shedding produces a forcing frequency in the in-line direction to that of the cross-flow direction. Experiments on flexible structures including field experiments (Vandiver et al, 2005, Vandiver and Jong, 1987), controlled laboratory experiments (Passano et al, 2010), and full scale observation of marine risers (Trim et al, 2005) have demonstrated that for long, flexible bodies, it is possible to excite different modes separately in the cross-flow and in-line directions. Assuming sinusoidal mode shapes for a string, the fundamental mode shape will look like a half sinusoid and the second would look like a full sinusoid as shown in Figure 6.

Dahl (2008) argued that the oscillation pattern for this circular cylinder would follow random and unrepeatable patterns. If one were consider the location $L / 4$, the orbital motion of the cylinder should exhibit a crescent or figure-eight motion, 
similar to those observed in the rigid cylinder, two-degree of freedom studies. In his free vibration experiments, Dahl noted that these figure-eight motions had a preferred direction where the cylinder would always move upstream at the extreme transverse motions. However, excitation of the second mode produces two transverse extremes, the other at $3 L / 4$ where the cylinder must move in the opposite direction. Due to the upstream motions at $L / 4$ and downstream motions at $3 L / 4$, it is suggested that a preferred direction of motion cannot occur if the resonance of both the first and second modes occur. Using PIV, Dahl confirmed that random oscillation patterns occur, and that these erratic motions caused wake formation to become extremely irregular, creating an imbalance of vortex shedding.

It is believed that the asymmetry of the in-line mode shape leads to the irregular motions because the cylinder cannot resonate in the in-line direction. In order to use a flexible cylinder with a natural frequency ratio of 2 , one must have a flexible cylinder where the in-line natural frequency is twice the cross-flow natural frequency, but the in-line mode shape is symmetric. Therefore; a flexible cylinder with an odd in-line mode shape is necessary to obtain regular oscillations in the in-line direction. This can be done for low mode flexible cylinders by tuning the natural frequencies of the flexible cylinder to meet a desired mode shape. At $L / 2$, crescent and figure-eight patterns were found to occur during lock-in between $V_{r}=6$ and $V_{r}=11$. Additionally the wake of the flexible beam cylinder was similar to those observed in the wake of a rigid cylinder.

In order to prove whether the cylinder is responding to fluid forces or whether the cylinder is being forced in motion by the resulting wake, a visual inspection or a measure of forces along the span-wise motion of the cylinder needs to be performed. Dahl (2008) argues that without a more detailed exploration of the entire flexible cylinders interaction with the fluid, it is not possible to make many conclusions 
about the motions of the cylinder. In order to address these issues, high-speed cameras and motion tracking software have been used to capture the span-wise motions of three flexible cylinders with in-line to transverse spatial frequency ratios of $1: 1,2: 1$, and $3: 1$ to observe irregular or regular orbital variations. 


\section{CHAPTER 3}

\section{Flexible Cylinder in Water Tunnel}

The free vibration of a flexible cylinder in a uniform flow acts as a fundamental experiment for understanding the motions associated with bluff-body, fluid interactions. Free vibration refers to the self-excited oscillation of a cylinder in a free stream due to instability of he flow. Free vibration experiments allow for observations to be made of physical vibrations of a system undergoing VIV, where observed measurements of motion response can be taken while the fluid flow is 2-D with respect to the cross-sectional shape of the cylinder.

In the experiments described in this chapter, the experimental focus was on seeing the effects of varied mode shape on the oscillatory motions of a flexible cylinder undergoing excitation in both in-line and and transverse directions. The

experiments were performed using visual observations to determine answers to the following questions:

1. How does mode shape affect VIV?

2. Does the interaction of different modes in in-line and cross flow directions lead to irregular or regular variations?

3. How is the cylinder wake affected by the modal excitation of the cylinder?

\subsection{Experimental Motivation}

A flexible cylinder excited by vortex shedding will have excitation in both in-line and transverse directions. Vortex shedding causes an excitation force at one times the fundamental frequency to occur in the lift direction and two times the fundamental frequency to occur in the drag direction, leading to an in-line 
to transverse natural frequency ratio of $2: 1$. In the case of a simply supported as shown in Equation 33, the system can either become bending or tension dominated,

$$
f_{\text {tensioned-beam }}=\sqrt{\frac{n^{4} \pi^{2} E I}{4 L^{4} M}+\frac{T n^{2}}{4 L^{2} M}} .
$$

where $n$ is the mode, $E$ is the modulus of elasticity of the beam, $I$ is the area moment of inertia of the beam cross section, $L$ is the beam length, $T$ is the tension force in the beam, and $M$ is the mass per unit length of the beam. In shallow water, the tension forces on a riser will be much smaller than compared to those experienced in deep water due to the weight of the riser material, when using comparable geometric and material characteristics.

In the cases examined within this thesis, the restoring forces due to tension are driven to zero, leaving the natural frequencies of a simply supported beam in Equation 34

$$
f_{\text {beam }}=\frac{n^{2} \pi}{2} \sqrt{\frac{E I}{L^{3} M}} .
$$

In the case of a bending dominated system as shown in Equation 34 with a circular cylinder, this 2:1 ratio becomes difficult to achieve because of the $n^{2}$ relationship between modal frequencies with the beam tending to respond with a natural frequency of 1:1.

In order to use a flexible cylinder to observe a natural frequency ratio of $2: 1$, one must have a flexible cylinder where the in-line natural frequency is twice the transverse natural frequency however, the in-line mode shape needs to be symmetric. This can be achieved by 'tuning' the flexible cylinder by molding a beam through the center of the cylinder. By creating a bending dominated system to mimic the natural frequency ratio of a string under tension, the natural frequency ratio is determined by the structural characteristics of the beam. 


\subsection{Experimental Procedure}

The first mode natural frequency is determined by Equation 34. For vortexinduced vibrations to occur, the frequency of vortex shedding needs to be near that or equal to the natural frequency of the cylinder. By setting the frequency of shedding as determined by a Strouhal number of 0.2 (Equation 1) equal to that of the natural frequency of the structure as determined by Equation 34, it is expected that these large amplitude motions will occur.

For each experiment and desired mode shape, the base and height dimensions of $I=\frac{b h^{3}}{12}$ are shown in Table 1 ,

\begin{tabular}{|c|c|c|c|}
\hline Beam & $\mathbf{1 - 1}$ & $\mathbf{1 - 2}$ & $\mathbf{1 - 3}$ \\
\hline \hline Material & Aluminum & Steel & Steel \\
\hline Dimensions & $\mathrm{b}=0.15$ & $\mathrm{~b}=0.32$ & $\mathrm{~b}=0.280$ \\
$(\mathrm{~cm})$ & $\mathrm{h}=0.32$ & $\mathrm{~h}=0.16$ & $\mathrm{~h}=0.063$ \\
\hline First Mode $f_{x}(\mathrm{~Hz})$ & 20.09 & 6.93 & 2.69 \\
\hline First Mode $f_{y}(\mathrm{~Hz})$ & 10.04 & 13.86 & 12.13 \\
\hline Excitation of Mode & $n=1$ & $n=2$ & $n=3$ \\
$\rightarrow f_{x}(\mathrm{~Hz})$ & 10.04 & 27.72 & 24.25 \\
\hline Ratio & 2 & 2 & 2 \\
\hline
\end{tabular}

Each beam was molded into the center of a $1 \mathrm{~cm}$ diameter, 17 in $(0.4318 \mathrm{~m})$ long flexible cylinder made of Clear Flex 50, a clear urethane rubber produced by Smooth-On. The fundamental frequency of each cylinder was also calculated to match the shedding frequencies determined by a known Strouhal number and flow velocity. Again, assuming sinusoidal shapes for a string, the fundamental mode shape will look like half a sinusoidal, the second harmonic will be a full sinusoidal cycle, and the third harmonic will look like one and a half cycles (Figures 7-8). 


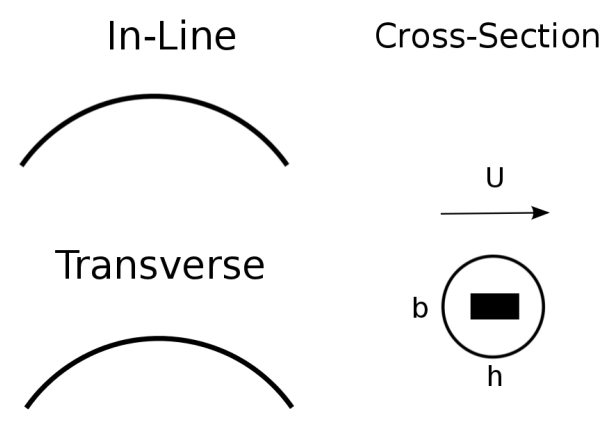

Figure 7: 1-1 In-line and Transverse Modes

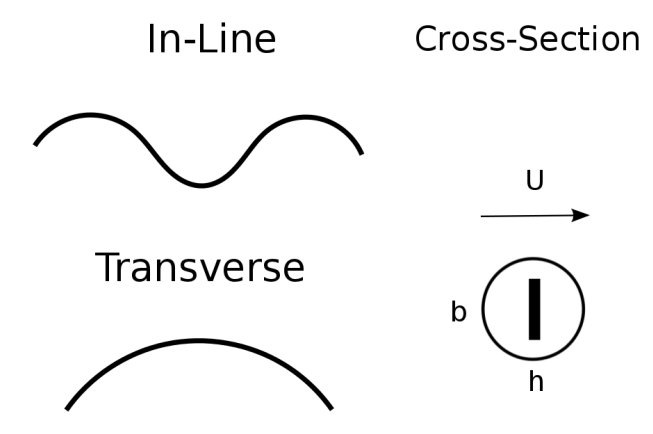

Figure 8: 1-3 In-line and Transverse Modes

To measure the cylinder motions, each beam was painted black, and small white dots at $1 \mathrm{~cm}$ intervals were added, providing up to 40 points along the length of the cylinder to be measured through video imaging and point motion tracking. Figure 9 shows the cylinder design.

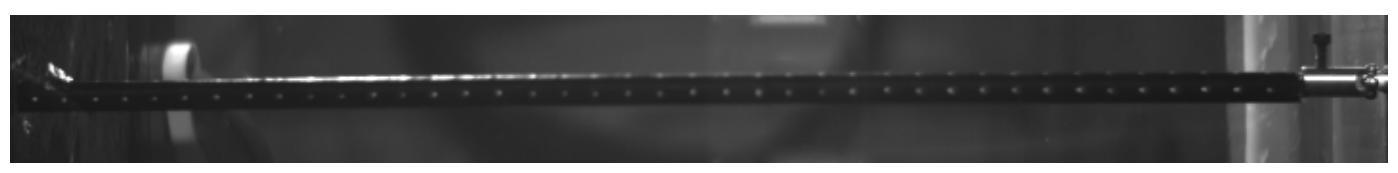

Figure 9: Cylinder Design 


\subsubsection{Cameras and Image Processing}

To visualize excitations, each flexible was placed inside the recirculating water tunnel located at the University of Rhode Island (URI) Narragansett Bay Campus. The water tunnel has a test section of 15 inches wide $(38.1 \mathrm{~cm}), 20$ inches deep $(50.8 \mathrm{~cm})$, and a length of 60 inches $(152.4 \mathrm{~cm})$ and tests were performed at speeds between 0.3 and $0.9 \mathrm{~m} / \mathrm{s}$. Flow speeds in the tank were determined using the calibrated motor frequency $(\mathrm{Hz})$ for the impeller driving the fluid through the channel. The impeller motor frequency was calibrated using a digital flow meter. The cylinder was mounted to a universal joint attached to a $\frac{5}{8}$ in acrylic falsebottom and to a sliding universal joint connected to a cross-wise beam to insure that simply supported beam end conditions were met. Upon reaching the desired flow speed, 12 seconds of data were captured to ensure a sufficient number of oscillation cycles.

To capture excitations made by the flexible cylinder, two Photron FASTCAM cameras were used. The FASTCAM-X 1280PCI 16k camera is capable of recording 500 fps at $1280 \times 1024$ pixels. A recording rate of 250 fps was used in each of these experiment in order to maximize illumination of the points being tracked is due to poor lighting conditions.Deflections of the beam were measured visually and reconstructed based on the cylinder motions observed in the camera frames. To capture motions in both planes, two cameras were setup as shown in Figure 10. 


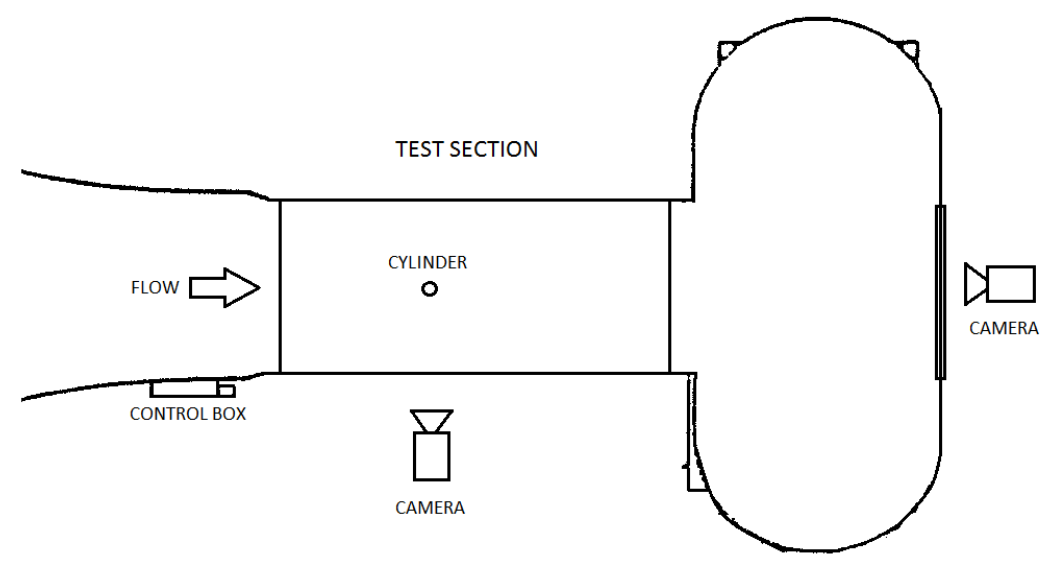

Figure 10: Camera Setup

FASTCAM software converted image sequences into .avi files for further processing in the motion analysis software ProAnalyst, developed by Xcitex. To use automatic tracking, a region was drawn around each individual point and labeled as a feature template. During automatic tracking, the software searches the region for the feature that is desired. Enabling sub-pixel accuracy attempts to estimate the best fit match to the feature template at a sub-pixel level. Additional image processing filters, such as binary thresholding, edge detection and sharpening allowed for better point tracking through the sequence of images. Binary thresholding converts the camera greyscale image and converts it to a binary image based on a threshold value defined for each sequence of images. A 5X5 Laplacian high pass filter was then used to find edges or point boundaries for points that only consist of a few pixels to enable better tracking.

These filters were applied to eliminate the effects of unusual lighting on the cylinder and unwanted particles that may interfere during the tracking process. In most cases, the application of filters was able to maximize the number of points 
for tracking, however in some cases, the number of pixels was not able to satisfy

minimum tracking requirements. Separate tests with altered threshold values were later run on points lost during initial tracking.

The calibration of $\mathrm{cm}$ to pixels was done within ProAnalyst from a known distance between two points along the span of the cylinder;the camera capturing in-line motion was calibrated at approximately 25 pixels per centimeter, and the camera capturing cross-flow motions was calibrated to approximately 17 pixels per centimeter. All point locations were referenced to the bottom left corner of each image after image rotation.

For every test-run, ProAnalyst created a text file detailing frame number, time, and $X$ and $Y$ coordinates of each point. All text files were loaded into MATLAB for further processing.

\subsection{Results}

\subsubsection{Excitation of the First In-line Mode}

For all observed motions along the length of the of the flexible cylinder, motions were regular, symmetric, and periodic as shown by a characteristic figure eight pattern in the upstream direction. Figure 11 shows the non-dimensional amplitude response of the center point of the cylinder as a function of reduced velocity. The normalized amplitude is defined as the average of the top ten percent of the local maxima from motion time history normalized by the diameter of the cylinder. Unlike the observations of the rigid cylinder experiments conducted by Dahl (2008) and Sarpkaya (1995), the data from the present study only occurs across a small range of $V_{r}$ as shown in Figure 12.

Reynolds numbers for the present study ranged from 3,000 to 8,000, while Dahl's experiments ranged from 11,00 to 44,000 and Sarpkaya reported a Reynolds number of 35,000 . The maximum non-dimensional amplitudes appears to match 
the second peak of Sarpkaya however, this comparison may be rather limited because nominal frequencies could have been provided by Sarpkaya.
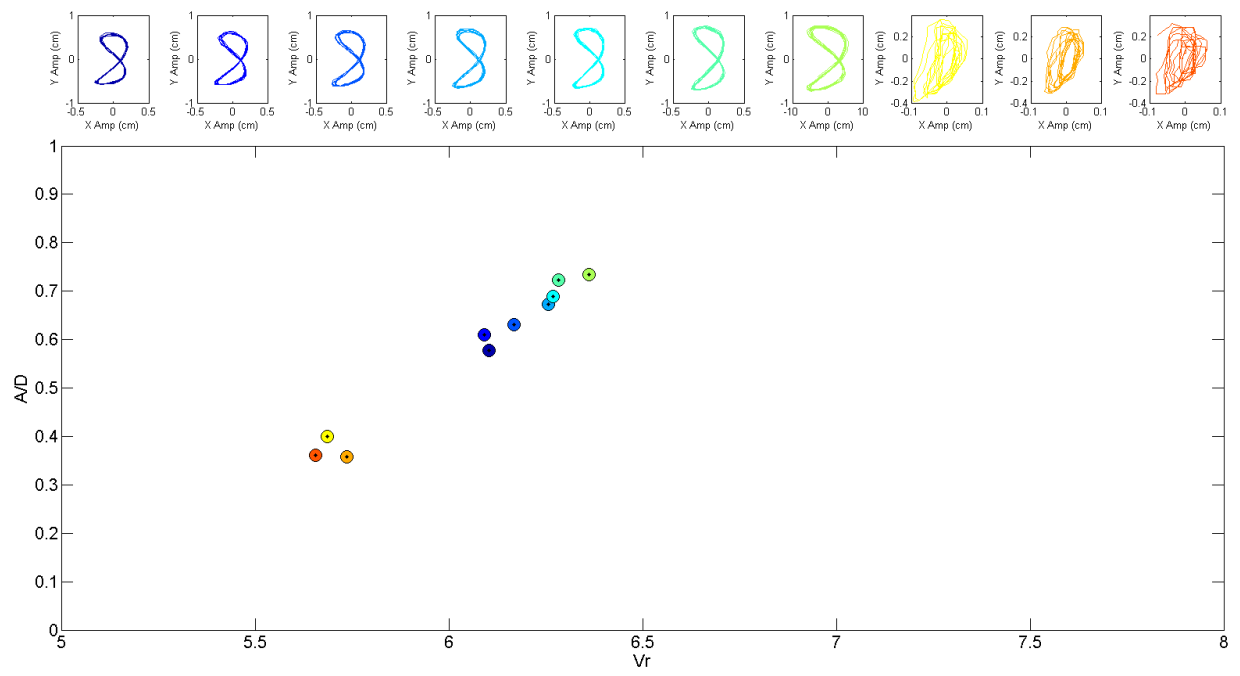

Figure 11: First In-line Mode: A/D vs Vr Present Study Only 


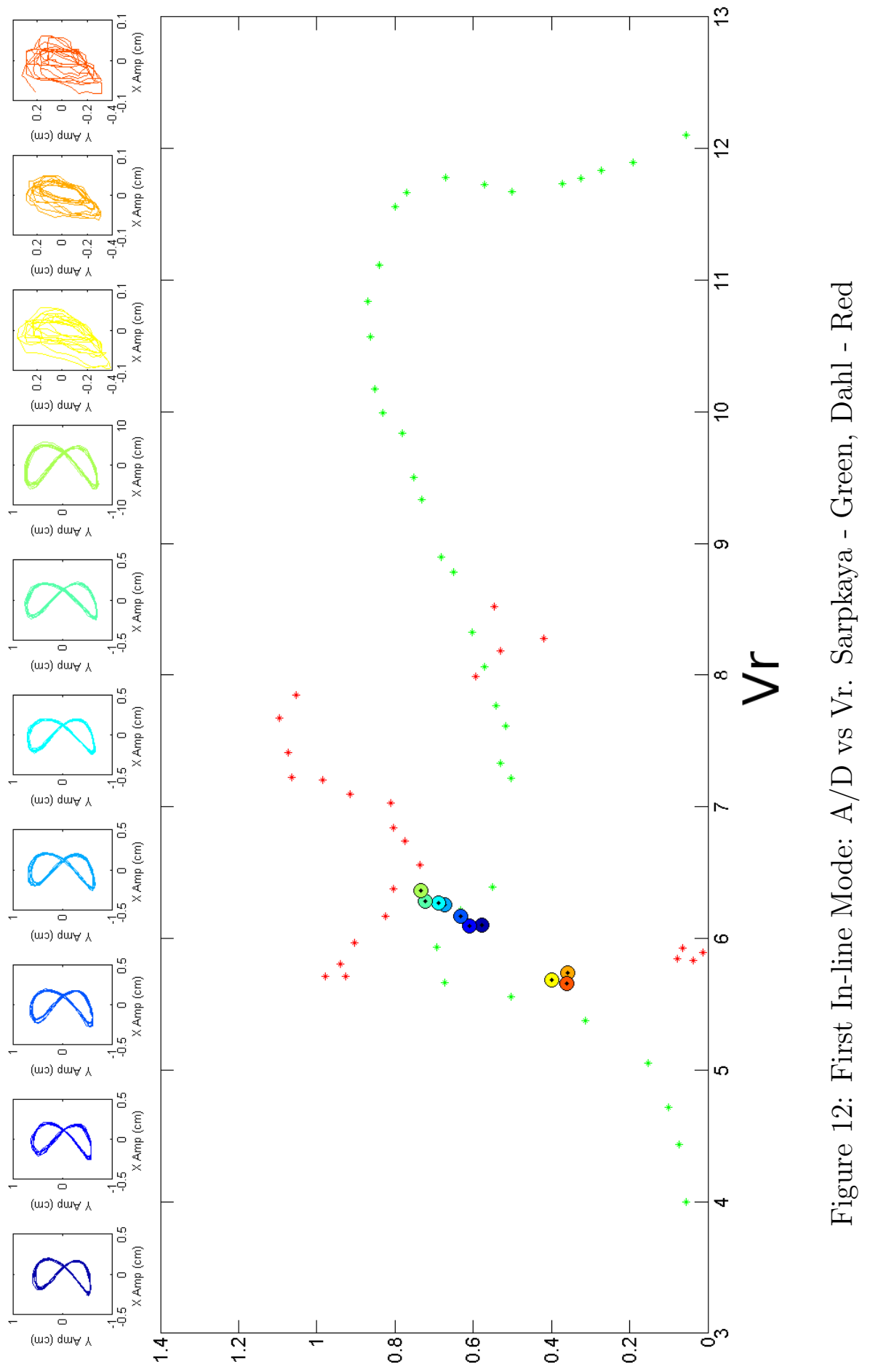

a/ $\forall$ 
The figure-eight patterns were observed for a range of $V_{r}=6.0$ to $V_{r}=6.4$ however, lower reduced velocities, (red, orange, and yellow) fail to create the same pattern, where random patterns of circles are seen instead. To further examine this case, time histories of amplitude and spectrograms are used to highlight maximum amplitudes and power spectral densities across the span of the cylinder. Figure 13 displays the the raw processing of the center point taken at $V_{r}=6.16$.
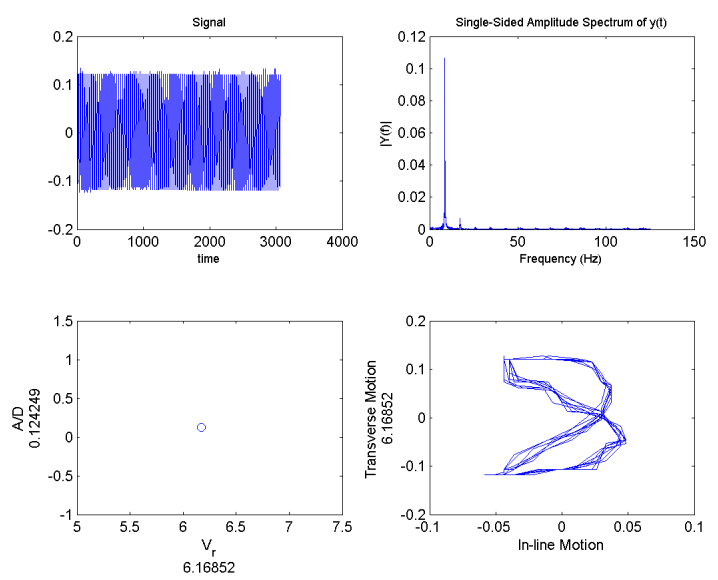

Figure 13: $\mathrm{Vr}=6.16$ Top left - raw data (cross flow), rop right - FFT, bottom left - A/D as a function of $\mathrm{Vr}$, bottom right - orbital pattern for 1-3 seconds

The top left image shows the center point cross-flow motions captured from ProAnalyst as a function of time. A Fast Fourier Transform (FFT) was used to isolate the effective frequency used to calculate the reduced velocity. The reduced velocity is plotted against the non dimensional amplitude (lower left). The lower right image shows the unfiltered Lissajous figure or orbital pattern of the motion for 1-3 seconds. For further analysis a time history of amplitudes and power spectral density plots are used to indicate any variance in frequencies during these experiments. Figures 13 and 14 show the in-line and transverse motion of the cylinder with a figure-eight orbital pattern at a reduced velocity of 6.16 . 

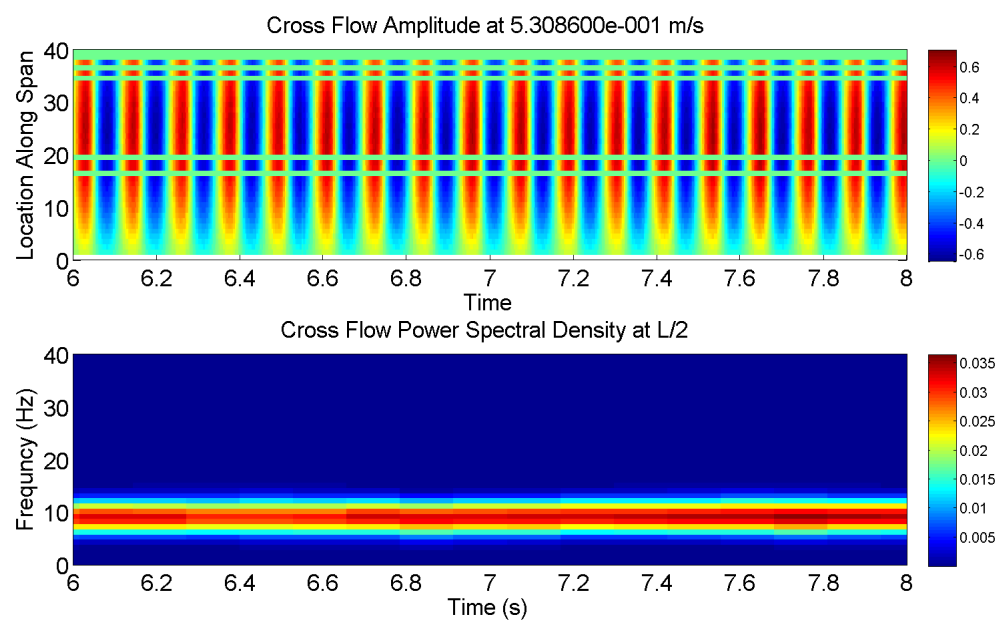

Figure 14: $\mathrm{Vr}=6.16$ Time history of amplitude and PSD of the CF Direction
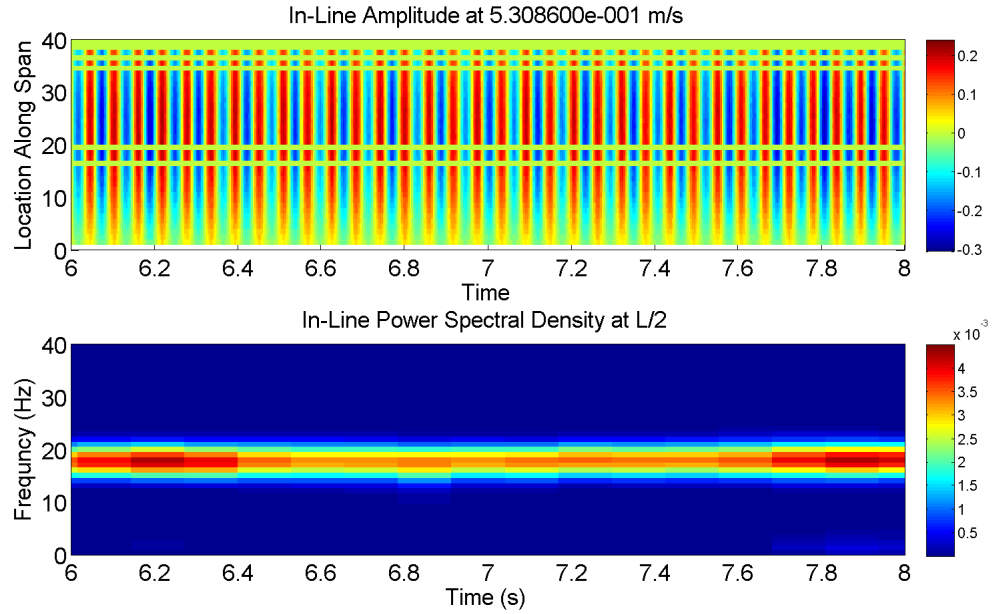

Figure 15: $\mathrm{Vr}=6.16$ Time history of amplitude and PSD of the IL Direction

The time history of amplitudes show that maximum amplitudes are followed by minimum amplitudes resultant of those seen in a standing wave. The 2:1 natural frequency ratio is confirmed with the in-line effective frequency of 18.8 and crossflow effective frequency of 9.6. The in-line and cross-flow effective frequencies indicated by the spectrograms are representative of all data collected within the 12 second time series.

While Figures 13- 14 show the amplitude and power spectral characteristics 
characteristic of a figure-eight pattern, a similar approach was used to examine why circular orbits were seen at lower values of $V_{r}$. Figure 15 shows the raw data, and Figures 16 -17 show the time history of amplitudes and frequency spectra from 1 second to 3 seconds at a $\operatorname{Vr}$ of 5.74 .
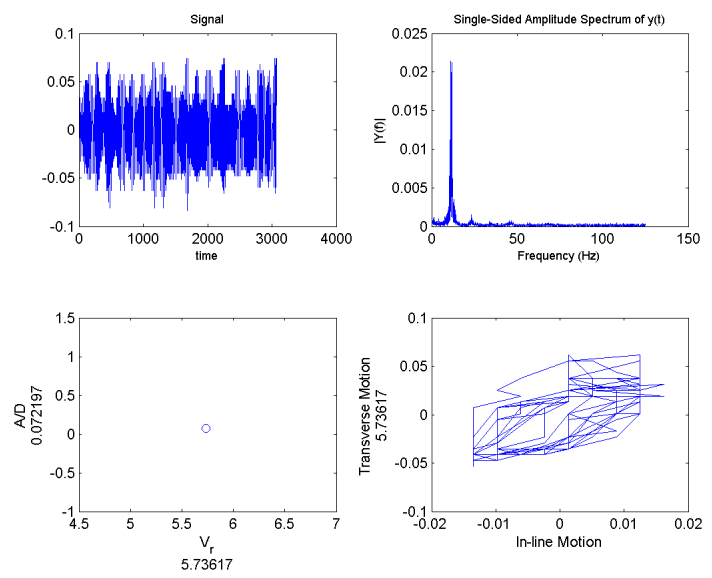

Figure 16: $\mathrm{Vr}=5.74$ Top left - raw data (cross flow), rop right - FFT, bottom left - A/D as a function of Vr, bottom right - orbital pattern for 1-3 seconds
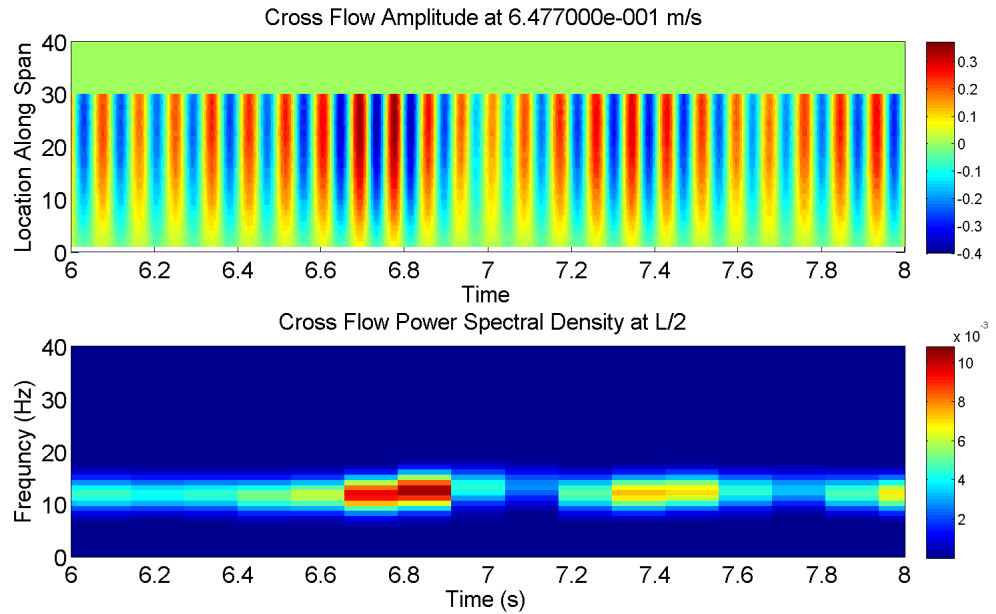

Figure 17: $\mathrm{Vr}=5.74$ Time history of amplitude and PSD of the CF Direction 

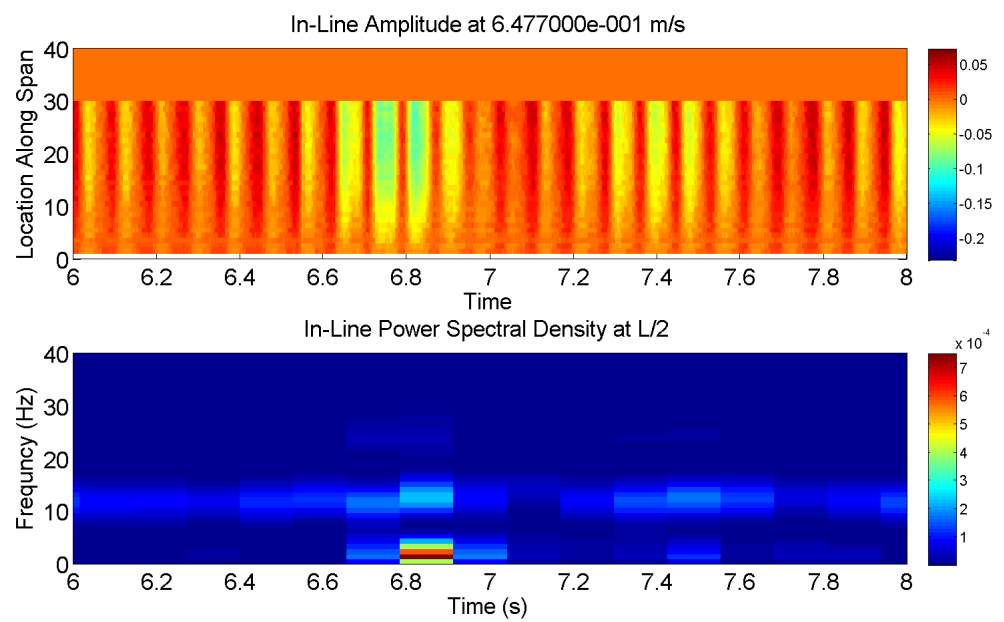

Figure 18: Vr=5.74 Time history of amplitude and PSD of the IL Direction

A similar time history pattern is seen as before however, there are small bands of relatively high amplitudes followed my a mean amplitude. This amplitude "beating" is suggestive of a shift in the mean in-line deflection, or drift of the cylinder where the circular motions appear to never achieve lock-in even though. The orbital patterns for both cases are shown below in Figures 18 and 19 .
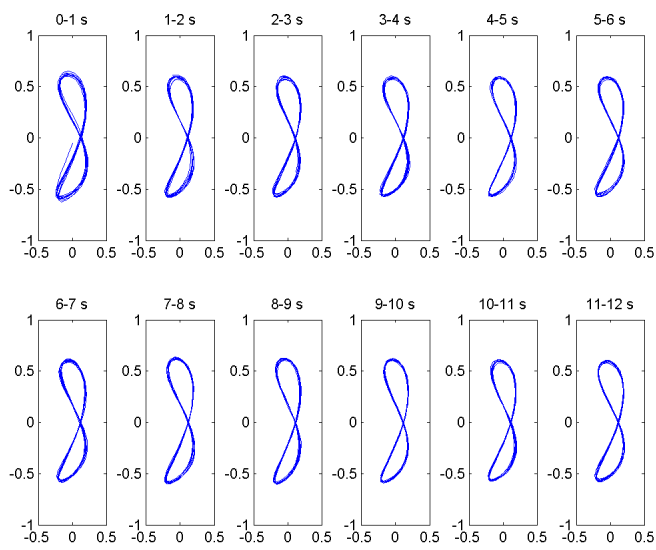

Figure 19: $\mathrm{Vr}=6.16$ orbital patterns $0-12 \mathrm{sec}$ 

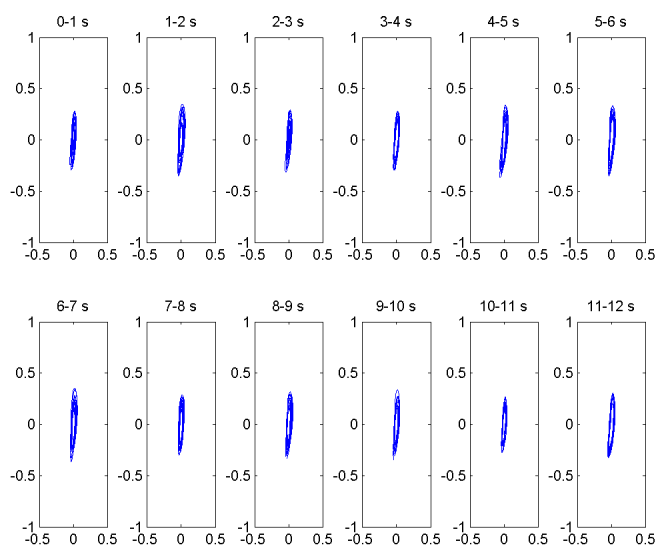

Figure 20: $\mathrm{Vr}=5.74$ orbital patterns $0-12 \mathrm{sec}$

\subsubsection{Excitation of the Second In-line Mode}

Dahl (2008) predicted that the orbital motions of a cylinder with the excitation of the second mode in the in-line direction would be irregular, random and unrepeatable. Any motion at the quarter point would most likely appear as a figure-eight moving in the upstream direction similar to what was found in rigid cylinder experiments and for the flexible cylinder with excitation of the first mode in both in-line and transverse directions. The same motion would be expected at three quarters point along the length of the cylinder, but in the downstream motion. The two competing motions of the asymmetric shape would result in random and non-periodic motions.

Data is again plotted terms in non-dimensional amplitude (A/D) and reduced velocity in Figure 20 with orbital patterns varying depending on $V_{r}$. Similar to the results for the excitation of the first mode in the in-line direction, a maximum non-dimensional amplitude of $\approx 0.8$ is reached. 


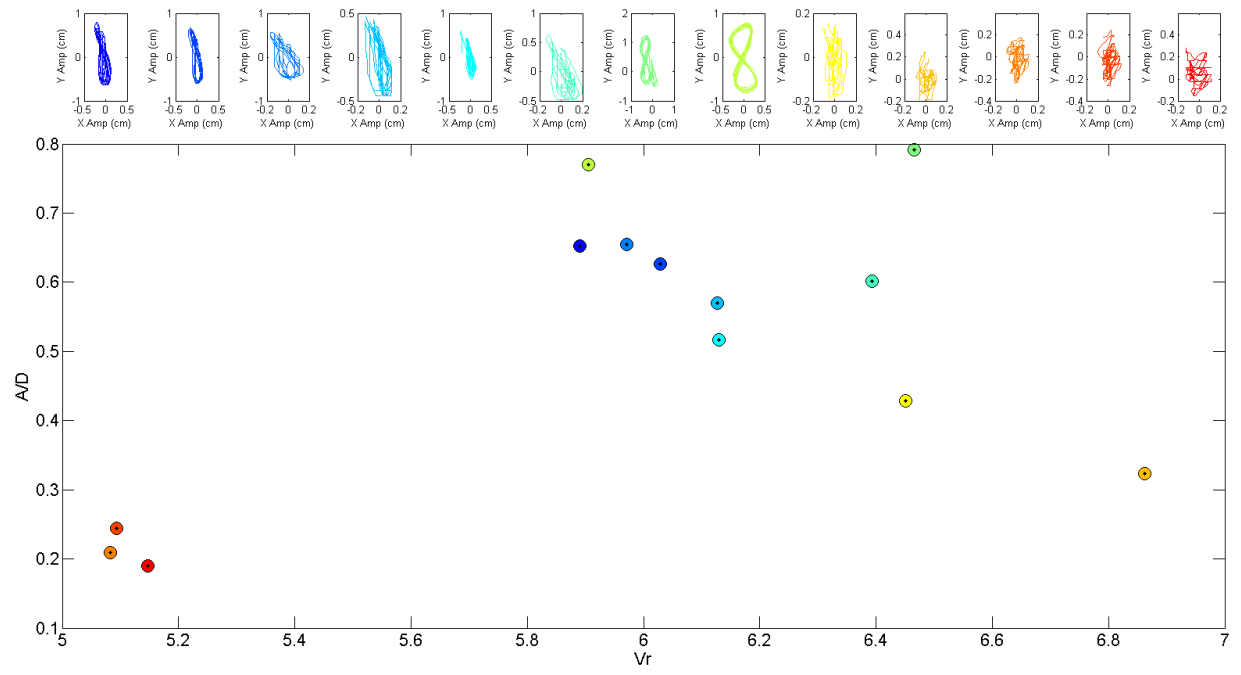

Figure 21: Second In-line Mode: A/D vs Vr Present Study Only

Each of the orbitals shown in Figure 20 are for the center point, L/2, location along the cylinder. At reduced velocities ranging from 5.8 - 6.2, a teardrop pattern appears, and at higher amplitudes for $V_{r}=5.9$ and $V_{r}=6.45$ (two green points), the cylinder begins to act as if the first mode was being excited instead of the second. Finally, random motions begin to appear for lower amplitude values but at both the lower and higher ends of the $V_{r}$ range.

Looking first at the teardrop shapes, Figures 21 and 22 show regions of interest when the cylinder was at $\mathrm{Vr}=6.13$. Again we see bands of where only a mean amplitude was reached and an inconsistency of one effective frequency. Bands of frequency are also observed in the cross-flow direction along with what appears to be a traveling wave as evidenced by the noted area at 1 second in Figure 22. These traveling waves result in reflections and over longer lengths can be seen more effectively. 

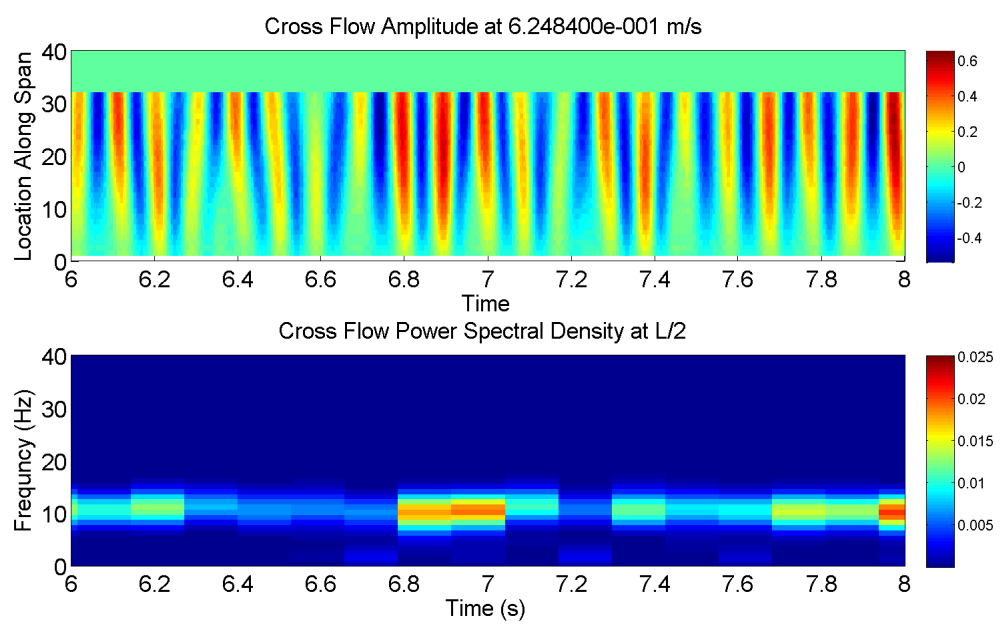

Figure 22: $\mathrm{Vr}=6.13$ Time history of amplitude and PSD of the CF Direction
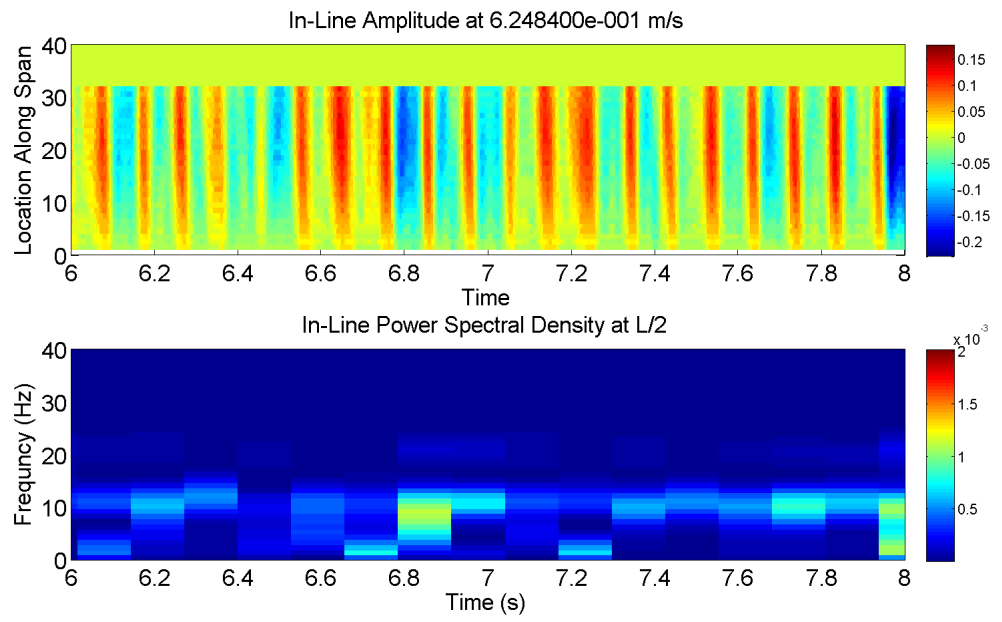

Figure 23: $\mathrm{Vr}=6.13$ Time history of amplitude and PSD of the IL Direction

When comparing the frequency responses of the teardrop shaped orbital patterns to those seen when observing figure-eights, there are significant differences. The in-line motion occurs with a frequency near $11 \mathrm{~Hz}$, while the cross-low motion occurs at about the same. Both the in-line and cross-flow motion appear to become excited by the same frequency which occurs in between the first natural frequency of the in-line direction $(6.93 \mathrm{~Hz})$ and cross-flow direction $(13.86 \mathrm{~Hz})$. At reduced velocities ranging between 5.8 and 6.2 , it appears that this relationship 
is found throughout the 12 seconds of data, however there is evidence that other frequencies are present and may become excited at different times such as those found when observing $V r=6.02$ in Figure 23 .
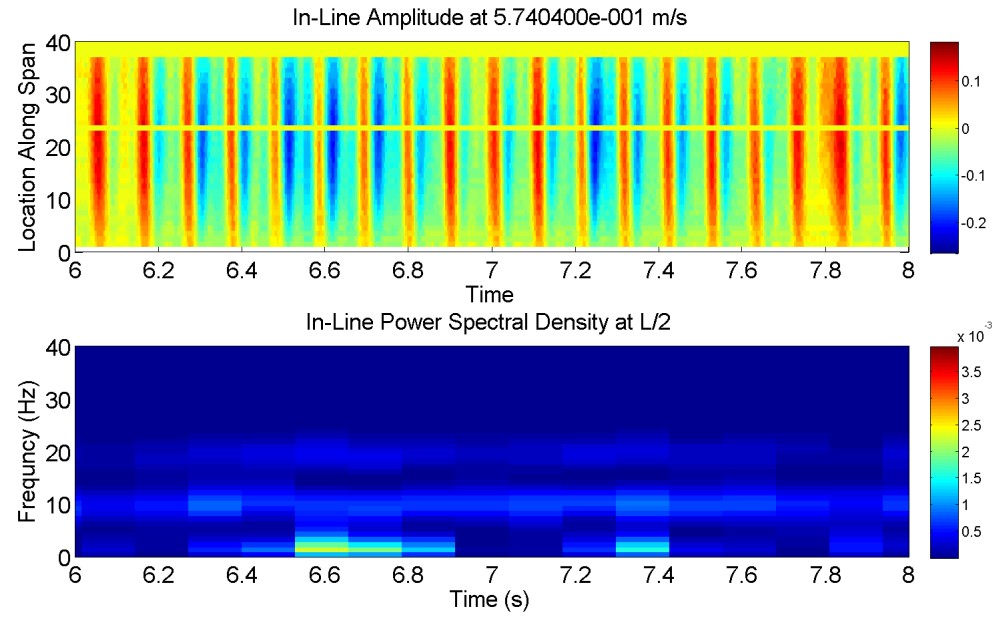

Figure 24: $\mathrm{Vr}=6.02$ Time history of amplitude and PSD of the IL Direction

While observing multi-frequency conditions, Kang and Jia (2013) found similar teardrop trajectory patterns when the frequency of the cross-flow vibration frequency favored an in-line vibration response similar to a natural frequency of one. They also noted that when there is a small increase in reduced velocity, the ratio begins to approach two resulting in figure-eight motions. This suggests that during this transition period (before and after figure eights are observed) that a "middle" frequency value is excited so other shapes such as teardrops are observed, rather than the traditional figure-eight or crescent shapes normally seen.

At higher reduced velocities, the variation of orbital patterns is directly related to the nearest structural mode. This mode switching, where the even in-line mode exhibits the excitation of twice the transverse frequency, but the spatial mode shape in-inline direction appears similar to the first structural mode shown in Figure 24. 


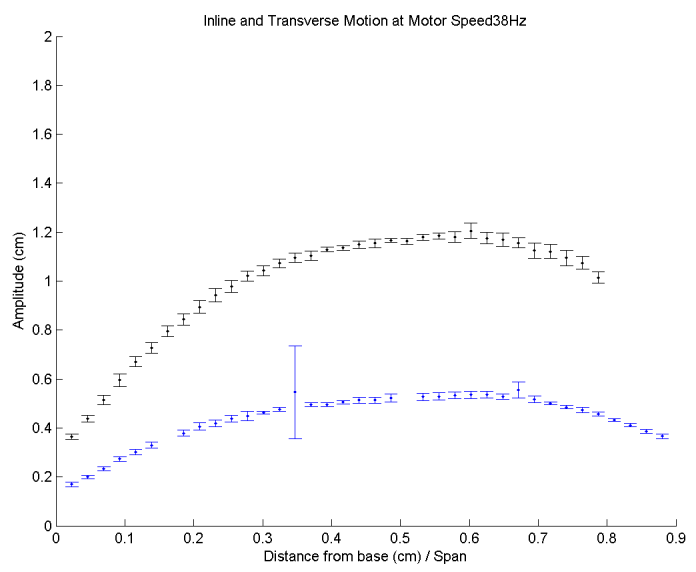

Figure 25: Vr=5.9 Spanwise shape of in-line (Black) and cross-flow (Blue) directions

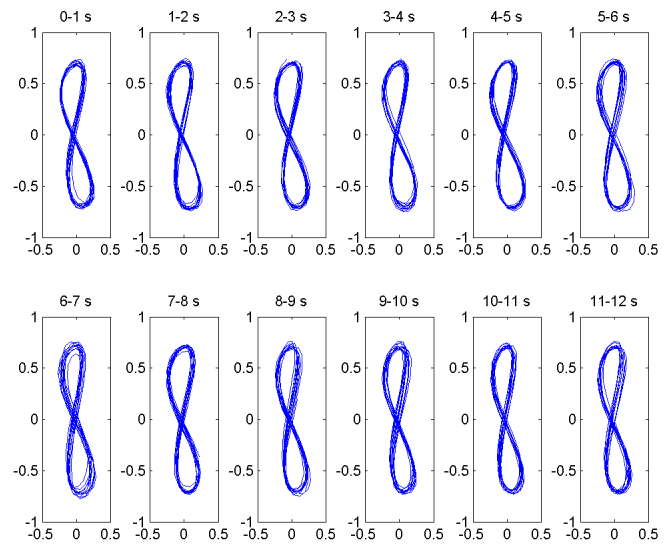

Figure 26: $\mathrm{Vr}=5.9$ orbital patterns $0-12 \mathrm{sec}$

The first mode can be seen in both the in-line (black) and transverse (blue) directions with the standard deviation of the top ten percent of the local maximum amplitudes against the non dimensional span of the cylinder. Figures 26 and 27 show the respective amplitude time histories and spectrograms in both the in-line and cross flow directions. At this reduced velocity, the cylinder exhibits motions that are consistent with the observed regular figure-eight shapes seen in when examining the first mode in both in-line and cross-flow directions. 

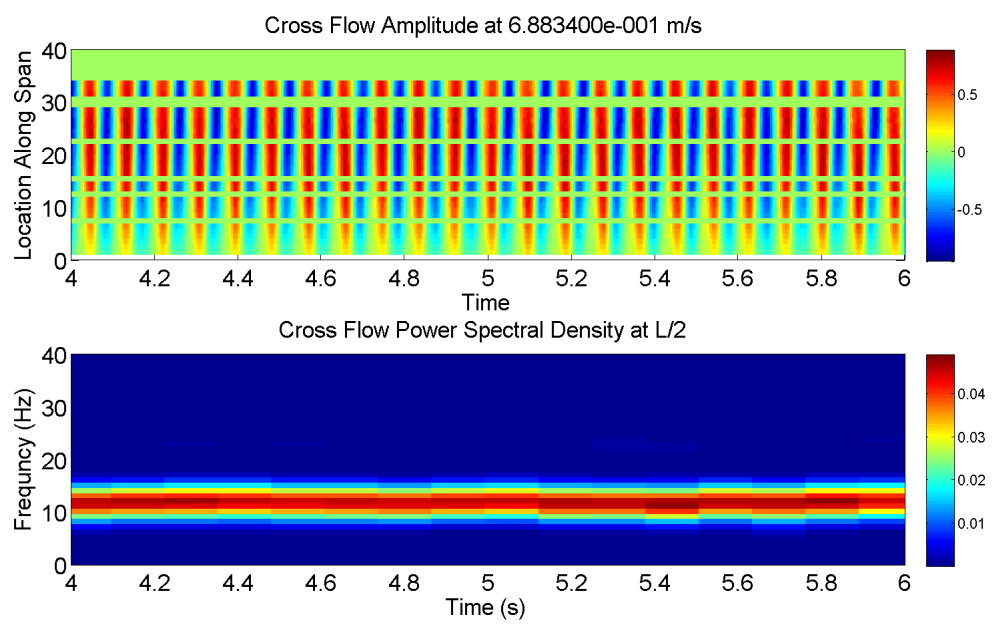

Figure 27: $\mathrm{Vr}=5.9$ Time history of amplitude and PSD of the CF Direction
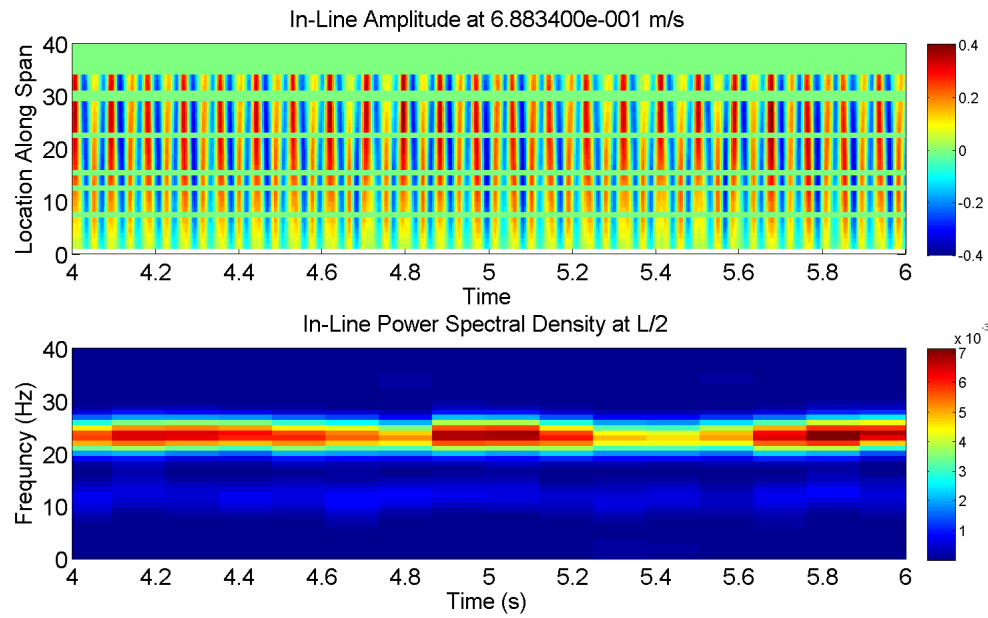

Figure 28: $\mathrm{Vr}=5.9$ Time history of amplitude and PSD of the IL Direction

Again, we observe standing waves in the in-line and cross-flow directions, similar to the 1:1 mode ratio cylinder as discussed in the previous section as is an effective frequency ratio of a $2: 1$.

During the examination of the time history of amplitudes, frequency spectra and time history of the orbital patterns (Figures 28-31) for the random patterns seen outside the range of reduced velocities ranging from $5.8-6.5$ (flow speeds greater than $0.7 \mathrm{~m} / \mathrm{s}$ ) the non-dimensional amplitude (A/D) vs point location 
divided by span $\left(Z_{i} / S\right)$ showed unexpected results (Figure 26) where the second mode is excited in the transverse mode, instead of the in-line mode. Due to the short span of the cylinder, what appear to be signs of traveling waves are actually an excitation of the second mode as evidenced by both the spanwise response and frequency spectra.

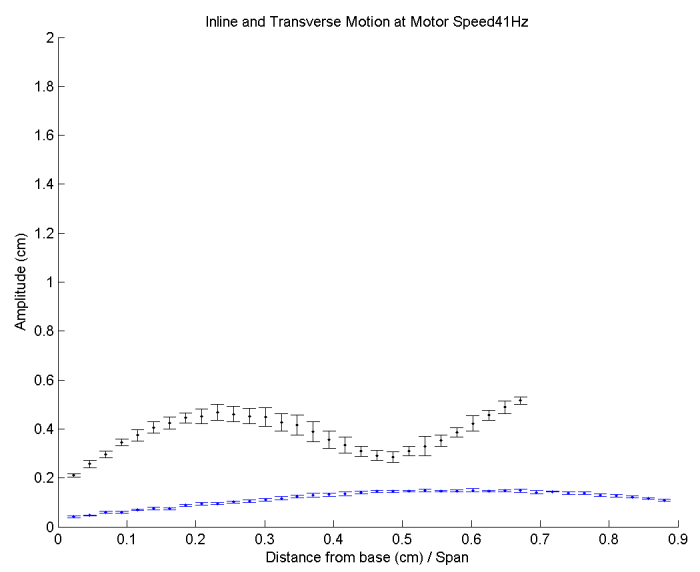

Figure 29: $\mathrm{Vr}=5.08$ Spanwise shape of in-line (Black) and cross-flow (Blue) directions
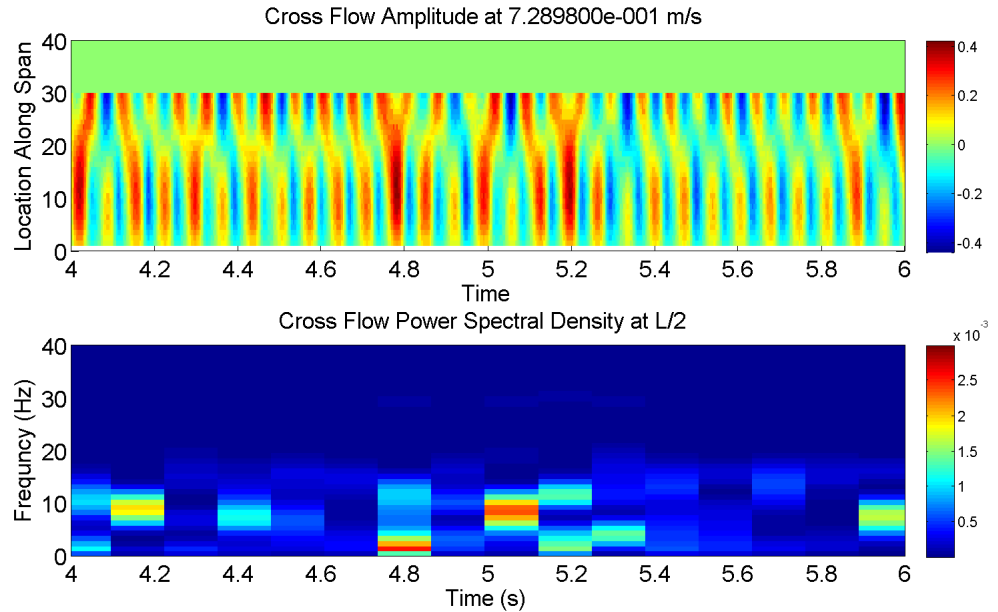

Figure 30: $\mathrm{Vr}=5.08$ Time history of amplitude and PSD of the CF Direction 

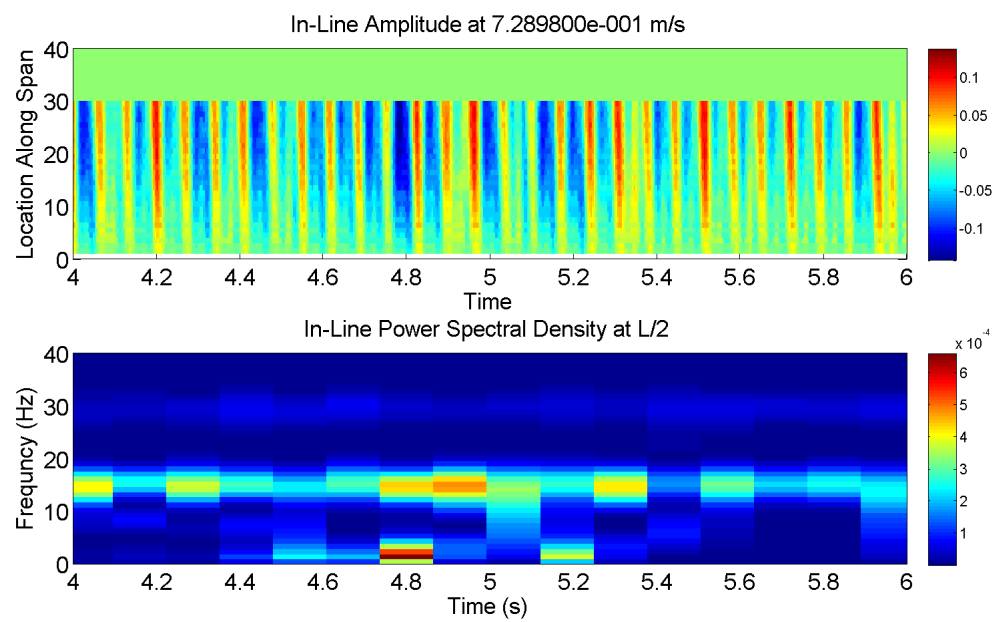

Figure 31: $\mathrm{Vr}=5.08$ Time history of amplitude and PSD of the IL Direction
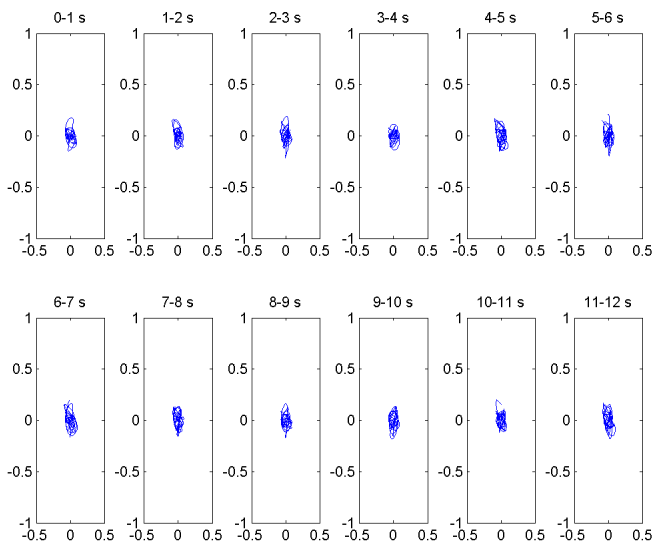

Figure 32: $\mathrm{Vr}=5.08$ orbital patterns $0-12 \mathrm{sec}$

Due to the mode shape change, a constant effective frequency was not witnessed in the cross-flow direction as seen in the in-line direction for several points along the span of the cylinder. The frequency bands seen in the in-line direction seem to have no effect on the orbital shape at $\mathrm{L} / 2$. It is important to note that effective frequencies in the in-line and cross-flow directions do appear to be near each other. 


\subsubsection{Excitation of the Third In-line Mode}

The third and final experiment was designed to test whether a symmetric mode shape, other than the first, would produce orbital results that were regular and periodic. Unlike the near linear relationship seen from the the 1-1 cylinder, no pattern is observed for the data produced by the 1-3 cylinder. All the data collected falls in between $V_{r}=5.12$ and $V_{r}=5.43$ and non-dimensional amplitudes of 0.65 and 0.705 (Figure 32). A comparison of all three data cylinders is shown in Figure 33.

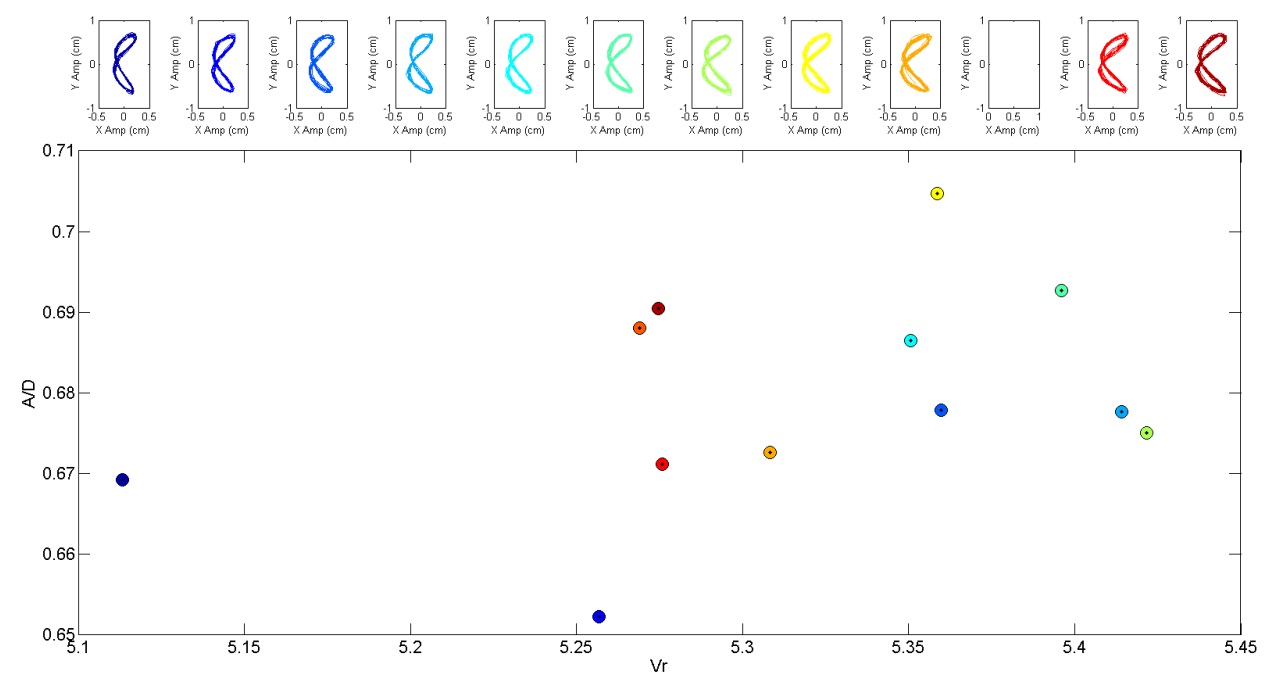

Figure 33: Third In-line Mode: A/D vs Vr Present Study Only 


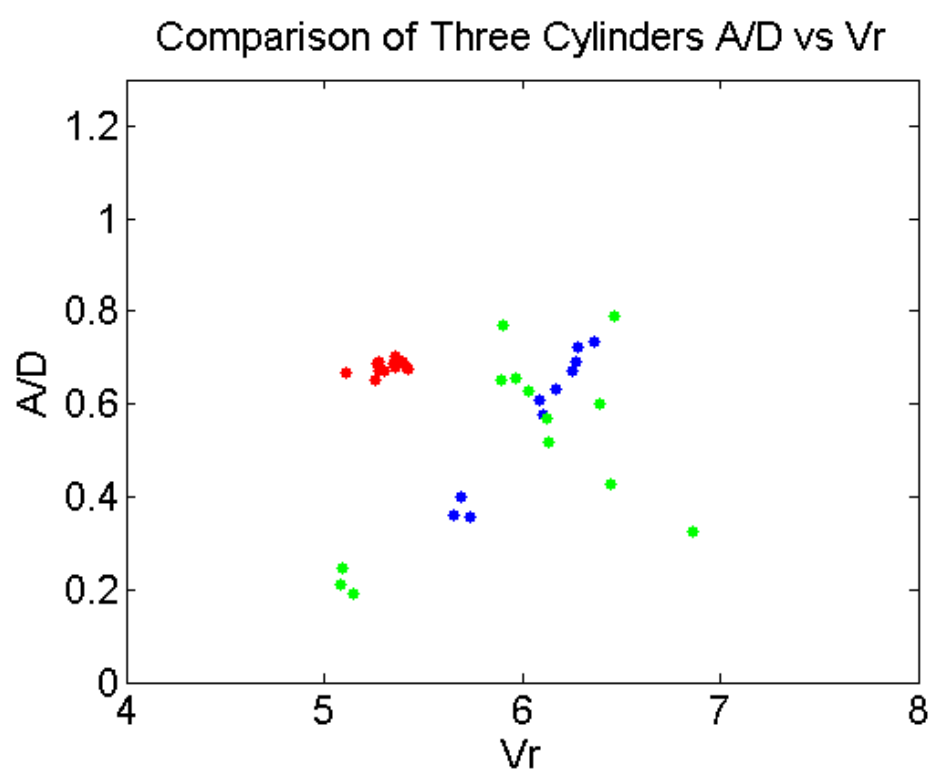

Figure 34: Comparison of $\mathrm{A} / \mathrm{D}$ vs $V_{r}$ for all three cylinders. Blue - Excitation of the first IL mode. Green - Excitation of the second IL mode. Red - Excitation of the third IL mode

Figure-eight patterns are present throughout this case however, the direction of curvature of the orbits has changed. Because the reduced velocities are much lower in this experiment, the figure-eights curve towards the right, whereas here they all curve towards the left in the 1-1 scenario.

Alternating maximum and minimum amplitudes within the amplitude histories for both the in-line and cross flow directions, Figures 34-35 follow the same pattern as the 1-1 case in which figure-eight patterns were witnessed. The spectrograms indicate that the in-line effective frequency is twice the cross-flow as expected, however the frequency from the $3 \mathrm{rd}$ mode is reached $(\approx 24 \mathrm{~Hz})$ shown below. 

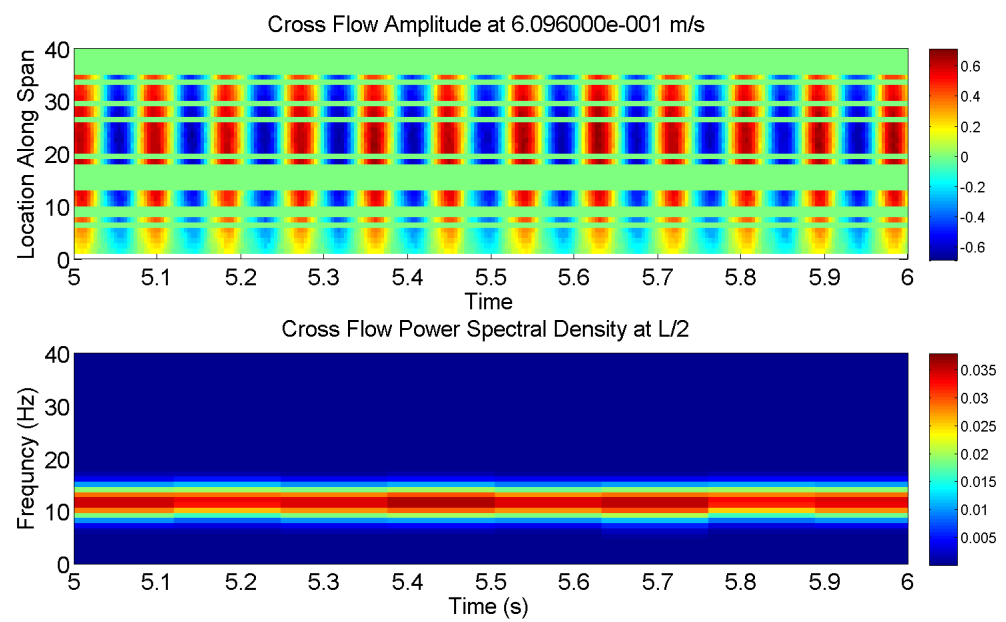

Figure 35: $\mathrm{Vr}=5.25$ Time history of amplitude and PSD of the CF Direction
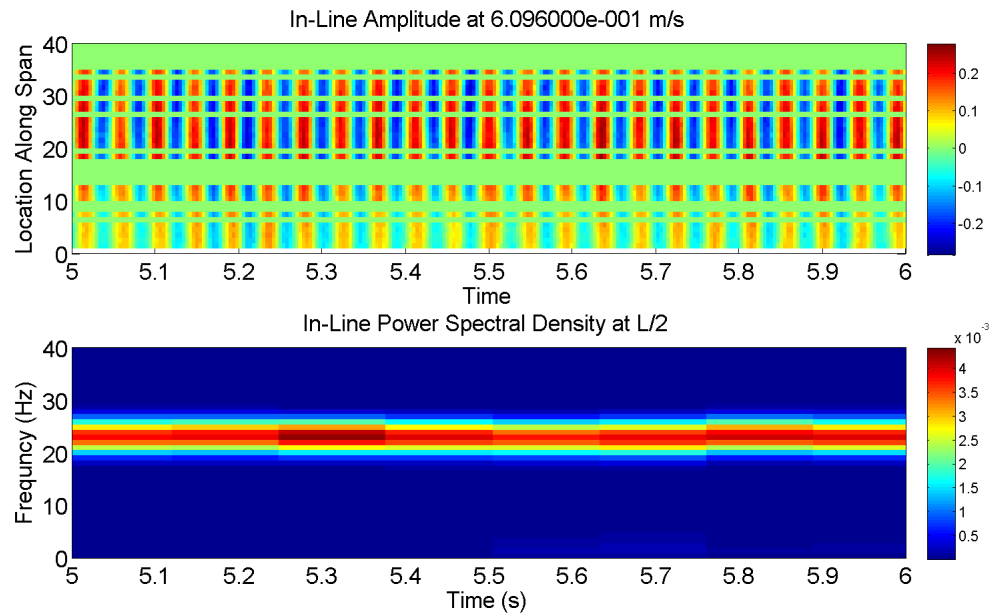

Figure 36: $\mathrm{Vr}=5.25$ Time history of amplitude and PSD of the IL Direction

The spanwise shape of both the in-line and cross-flow directions does not show the 3rd mode shape during any of these experiments.

\subsection{Discussion on Flexible Cylinder Experiments}

The structural characteristics of the three cylinder experiments play an important role in the observed behavior. In the case of the first cylinder (1-1), very regular cylinder motions were observed. A comparison of results from this cylinder can be made to those observed by Dahl (2008). Figure 10 shows the measured 
cross $=$ flow $A_{y} / D, A_{x} / D$ of the observed orbit shapes. The figure eight motions observed resulted amplitudes in excess of $A_{y} / D=1.5$ and $A_{x} / D=0.5$ very similar to those observed by Dahl near a $V_{r n} \approx 8$ in Figure 36 below.

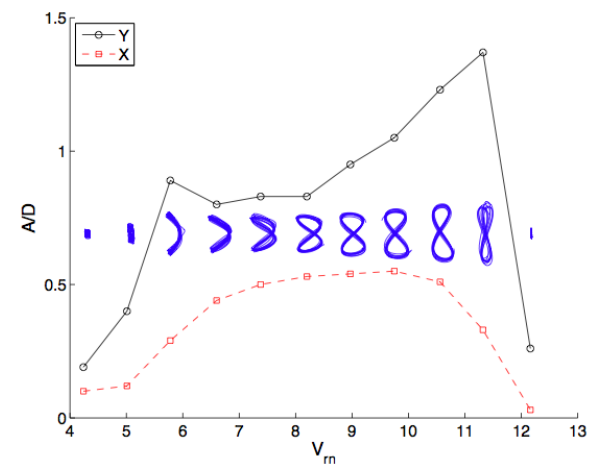

Figure 37: Non-dimensional amplitudes and orbit shapes for flexible beam cylinder motions at the midpoint of the beam Dahl(2008).

The outlying three data points values (Figure 11) can also be matched with amplitude values observed by Dahl. The exact figure-eight shapes and respective non-dimensional values seen after $V_{r n}=8$ were never reached in the current experiment. The motions observed in this thesis and those by Dahl in Figure 36 are very similar to the rigid cylinder free vibration experiments with $f_{n x} / f_{n y}=1.9$ seen in Dahl (2008).

The theory presented by Dahl (2008) in Chapter 2 of this thesis argues that the oscillation patterns observed from this cylinder would be random and unrepeatable. However, the data collected (Figure 20) in in this set of experiments produced three unique patterns:

1. Repeatable patterns with teardrop shape,

2. Repeatable patterns with figure-eight shape,

3. Random and unrepeatable patterns. 
The data points representing the repeatable teardrop and figure-eight patterns closely matches the first peak of Sarpkaya's (1995) rigid cylinder experiments as shown in Figure 12. The teardrop patterns seen before the figure-eights were also observed in the elastically mounted rigid cylinder experiments of Kang and Jia (2013) during the transition to figure-eight patterns with similar results being observed in this thesis. For this particular case, $V_{r n} \approx 2$, while values less than 1 were observed to produce egg or teardrop shaped patterns in Kang and Jia (2013). This difference is due to a difference in mass ratios of the cylinder. The teardrop patterns were witnessed when the excitation of the first mode in the cross-flow and in-line directions were observed and an effective frequency of both the in-line and cross-flow motions were approximate to each other. Spectrograms show evidence of other frequencies less than $5 \mathrm{hz}$ being excited, however only in the in-line direction. Figure eight patterns occur when the frequencies reach the $2: 1 f_{x} / f_{y}$ ratio as seen in experiments with the first cylinder but quickly disappear when $V_{r}$ is changed. When closely examining the amplitude time histories and spectrograms of the first two patterns, the results are comparable to those when discussing the first cylinder however, at flow speeds greater the $0.7 \mathrm{~m} / \mathrm{s}$, the patterns begin to appear random as previously suggested.

Figure 28 shows multiple mode excitation along the span of the cylinder, because the shedding frequency is between both of the frequencies associated with the two modes. At lower speeds, only the first mode is excited in either the inline or cross flow directions, but as the speed was increased, excitation of the second mode in the cross-flow direction while the first mode remains in the inline direction. The observed frequencies in these cases appear to be relatively close to one another which, may indicate that if speeds were increased further first mode may eventually disappear, exciting the second mode in both directions. 
The natural frequency difference as indicated by Table 1 is $\approx 7 \mathrm{~Hz}$, however if the natural frequencies of the cylinder are much further apart, the shift to the second mode may become much more difficult to achieve. The frequency variation seen in the in-line motion spectrograms may provide evidence for this to occur. Further observations of the orbital patterns along the span confirm that the second mode was excited, with small amplitude values at the node, and increasing values as the anti-nodes are reached.

Results in Figures 32 and 33 indicate that the data from the third cylinder was collected within a small region of $V_{r}$. This explains why the the non-dimensional amplitudes of the orbital patterns are do not change. Due to this small range, it becomes difficult to compare these results to those observed when looking at the first two cylinders. A larger $V_{r}$ range would probably yield similar results to those seen when examining the first cylinder, however further experiments will be required to confirm this. 


\section{CHAPTER 4}

\section{Conclusions}

\subsection{Overview}

Long risers with structural characteristics similar to strings will inevitably have the possibility of excitation of modes with a 2:1 ratio along the length. When the transverse mode of the cylinder corresponds to the Strouhal frequency of vortex shedding, the resonant behavior of the structure will lead to large amplitudes and dual resonance in both the in-line and cross-flow directions. The fundamental theme throughout this work has been the effect of three different mode shapes in the in-line direction on a flexible cylinder in a free stream.

The following sections outline the most important contributions of this thesis. Three experiments have been performed, resulting in a number of observations concerned with vortex-induced vibrations. An analysis of the orbital patterns created by a flexible cylinder in two degrees of freedom, has provided a new step into understanding vortex-induced vibrations and their effect on marine riser design. This study, by no means, offers a complete solution to the problem. Recommendations for future work are also made in the last section, primarily concerned with relevant continuation of the studies presented in this thesis or concerned with improvements upon the presented studies.

\subsubsection{1-1 Mode Ratio}

By tuning the in-line natural frequency of the flexible cylinder to occur with an odd mode shape similar to the transverse mode shape, regular periodic motions of the flexible cylinder were obtained. Although this study maintained focus on such a small range of reduced velocities, the oscillations of the tuned flexible cylinder were very similar to those observed by Dahl(2008) and Sarpkaya(1995) for the free 
vibrations of a rigid cylinder and by Dahl(2008) for a flexible cylinder. The wake behind the tuned flexible cylinder is presumed to be very regular.

\subsubsection{1-2 Mode Ratio}

Initial predictions and observations by Dahl (2008) suggested that a cylinder maintaining a 2:1 frequency ratio with a 1-2 mode ratio would result in irregular and random orbital patterns. At $L / 4$, Dahl predicted that the cylinder must undergo the figure-eight pattern observed with rigid cylinders and should have an upstream figure-eight orbital. However, at $3 L / 4$ the cylinder would undergo the same pattern but in the downstream direction. Over the entire span of the cylinder, the asymmetric second mode shape would cause irregular motions to occur with no sustainable periodic motion.

The observations of the cylinder with the 1-2 mode ratio phenomena produced more complicated results than what was anticipated. Plotting the mean of the top $10 \%$ of the amplitudes for each point along the cylinder indicate that the first mode was being excited in both the cross-flow and in-line directions when $V r=5.9$. The natural frequencies $f_{x}=6.9 \mathrm{~Hz}$ and $f_{x}=13.8 \mathrm{~Hz}$ of the beam, were not observed in much of the data, rather an effective frequency of $\approx 11 \mathrm{~Hz}$ was excited. This resulted in the first mode in both the in-line and cross-flow being excited. Instead of irregular patterns, teardrop shapes were observed suggestive of a unique flow in the cylinder wake. The results from this cylinder suggest that either vortices or one vortex pair per cycle is being shed to one side of the cylinder. Wake visualization is recommended to explore if this wake pattern occurs.

The isolation of specific modes was determined to be to be rather difficult due to mode sharing; the ability of the cylinder to be excited by different combinations of modes even though the natural frequency ratio remained at 2:1. Further studies are recommended to examine these transitions. 


\subsubsection{1-3 Mode Ratio}

The design of another symmetric mode in the in-line direction did in fact produce regular and periodic patterns along the entire length of the cylinder for each flow speed tested. Also found was that the excited frequency was near the third natural frequency, but the 3rd structural mode was not observed which, may have been due to superposition of the mode shapes. However, we did see the curvature of the figure-eight pattern lean to the right as opposed to those seen in the 1-1 case. Again, the wake for this case is expected to be very regular.

\subsection{Future Work}

Experiments can always be improved by using better equipment or better experimental conditions, however several aspects of this study have raised more questions important to the topic of vortex-induced vibrations. While much of this work may provide insights into how mode shape effects VIV and the orbital patterns that are observed, only the groundwork has been laid.

The effects of glare from additional lighting used to capture images were able to be minimized during image processing. In some cases, the image processing resulted in some points being only a few pixels which were unable to be tracked through the entire image sequence. Additional motion tracking was performed with variations made to threshold and edge detection filters when large groups of points

were unable to be tracked through the image sequence. Any points consisting of only 1-2 pixels were unable to be tracked consistently.

At high flow velocities, several sources of error were noted during data acquisition:

1. Supports were added when the acrylic false bottom failed to remain in place,

2. Bubble formation at the free surface, 


\section{Drop in water level.}

As higher flow speeds were reached, the acrylic false bottom needed additional support to remain in place. Threaded rods were placed to the sides in front of and behind the cylinder in the best positions to not interfere with the experiment. It remains unknown as to whether these rods had any ill-effect on the results.

Bubble formation complicated motion tracking for points of interest near the free surface and continued to get progressively worse as a significant drop in water level was noticed at flow speeds greater than $0.7 \mathrm{~m} / \mathrm{s}$. The drop in water level resulted in the failure to maintain complete submergence of the cylinder and data acquisition at desired speeds above $0.8 \mathrm{~m} / \mathrm{s}$. For further studies, it is suggested that the cylinder design and experimental setup be changed so that the cylinder can be horizontal in the water column rather than vertical.

Data collection over a longer period of time would enable a more advanced frequency analysis to examine non-linear effects, however that will be based on how and what image capturing software is used. Resolution of the image can be very important, and capturing data at a greater frames per second would require large amounts of memory and lighting.

A larger $V_{r}$ range when acquiring data for the third mode excitation would probably yield similar results to those seen when examining the first cylinder however, further studies are needed. The effects on cylinder wake have only been hypothesized, and requires further wake visualization techniques such as digital particle image velocimetry (dPIV), similar to the methods used by Dahl (2008), but across the length of the entire cylinder, not just points of maximum amplitude. 


\section{LIST OF REFERENCES}




\section{APPENDIX A}

\section{Appendix A}

Calibration Chart for the Circulating Water Tunnel

\begin{tabular}{|c|c|}
\hline Motor $\mathrm{Hz}$ & Flow Speed $(\mathrm{m} / \mathrm{s})$ \\
\hline 24 & 0.42926 \\
\hline 25 & 0445 \\
\hline 26 & 0.4699 \\
\hline 27 & 0.4826 \\
\hline 28 & 0.50292 \\
\hline 29 & 0.51308 \\
\hline 30 & 0.53086 \\
\hline 31 & 0.55372 \\
\hline 32 & 0.57404 \\
\hline 33 & 0.58674 \\
\hline 34 & 0.6096 \\
\hline 35 & 0.62484 \\
\hline 36 & 0.6477 \\
\hline 37 & 0.66294 \\
\hline 38 & 0.68834 \\
\hline 39 & 0.70485 \\
\hline 40 & 0.71628 \\
\hline 41 & 0.72898 \\
\hline 42 & 0.7493 \\
\hline 43 & 0.76962 \\
\hline 44 & 0.78232 . \\
\hline
\end{tabular}




\section{APPENDIX B}

\section{Appendix B}

First Mode In-line and First Mode Cross-Flow (1-1 Cylinder Results)

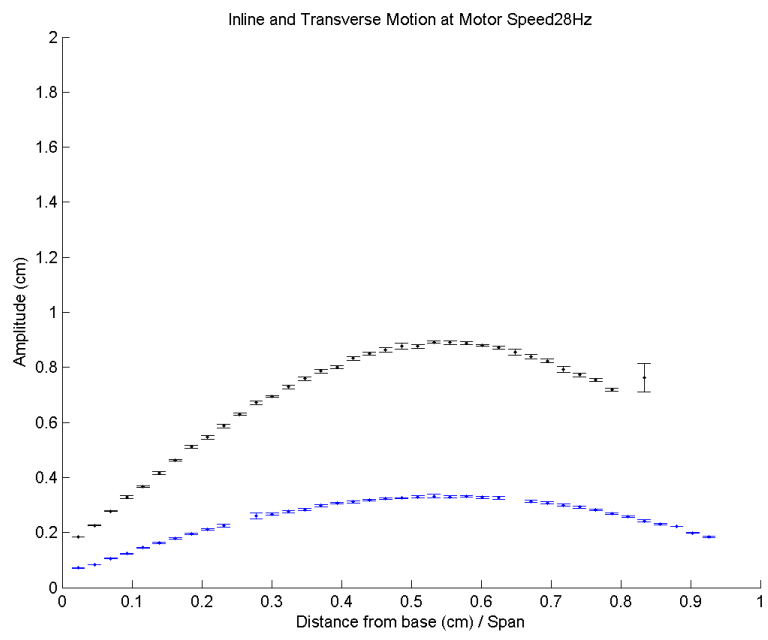

Figure B.1: $\mathrm{Vr}=6.1(\mathrm{U}=0.50 \mathrm{~m} / \mathrm{s})$, Spanwise shape of in-line (Black) and crossflow (Blue) directions
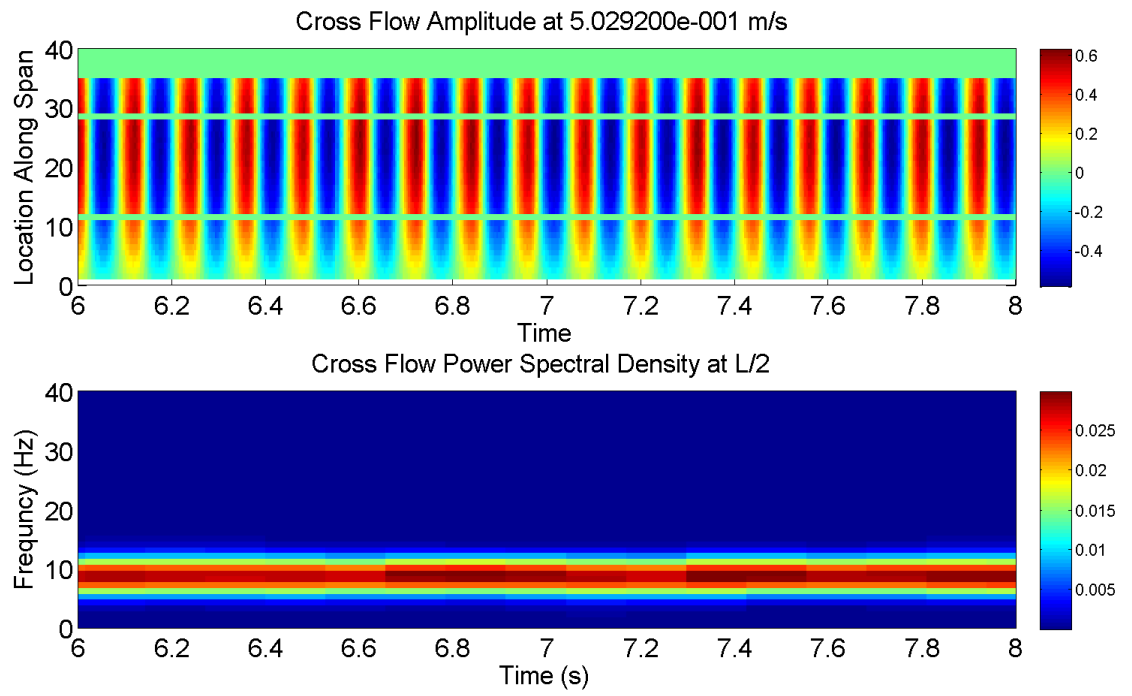

Figure B.2: $\mathrm{Vr}=6.1(\mathrm{U}=0.50 \mathrm{~m} / \mathrm{s})$, Time history of amplitude and PSD of the CF Direction 

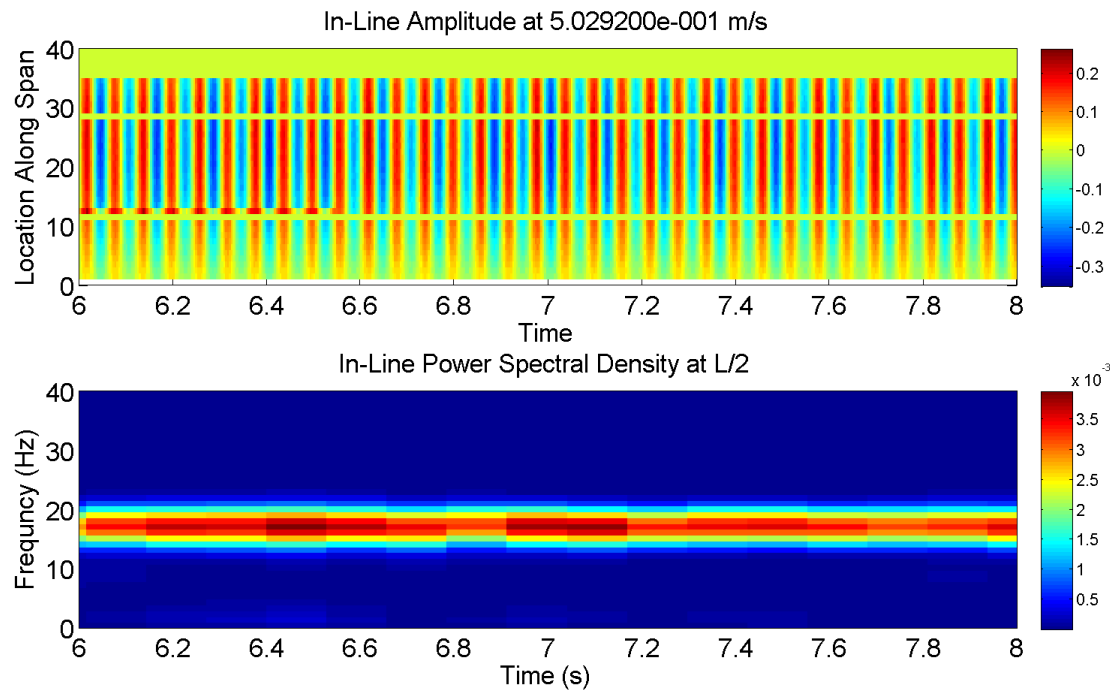

Figure B.3: $\mathrm{Vr}=6.1(\mathrm{U}=0.50 \mathrm{~m} / \mathrm{s})$, Time history of amplitude and PSD of the IL Direction
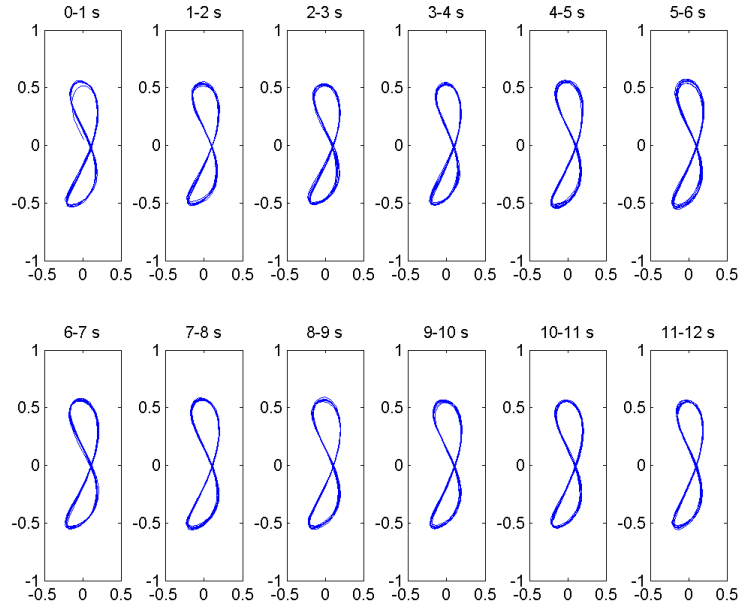

Figure B.4: $\mathrm{Vr}=6.1(\mathrm{U}=0.50 \mathrm{~m} / \mathrm{s})$, orbital patterns $0-12 \mathrm{sec}$ 


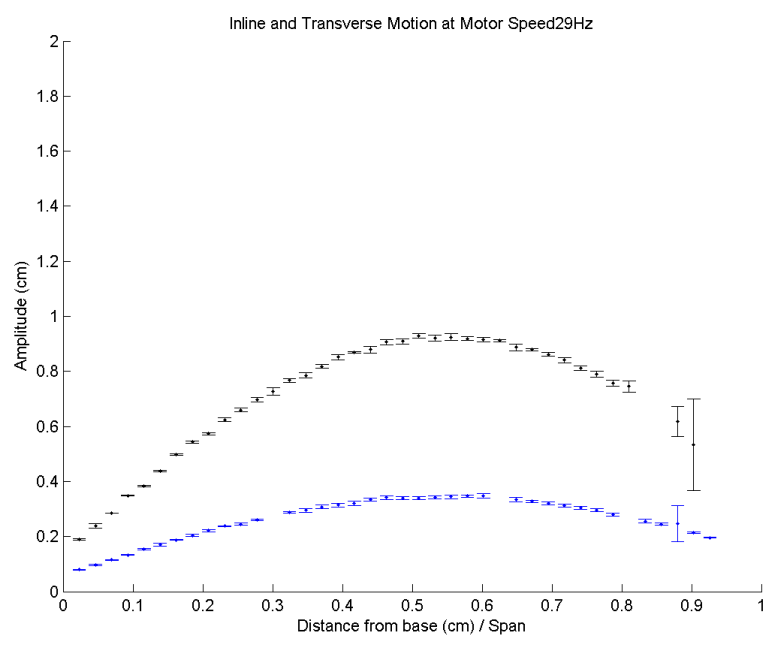

Figure B.5: $\mathrm{Vr}=6.09(\mathrm{U}=0.51 \mathrm{~m} / \mathrm{s})$, Spanwise shape of in-line (Black) and cross-flow (Blue) directions
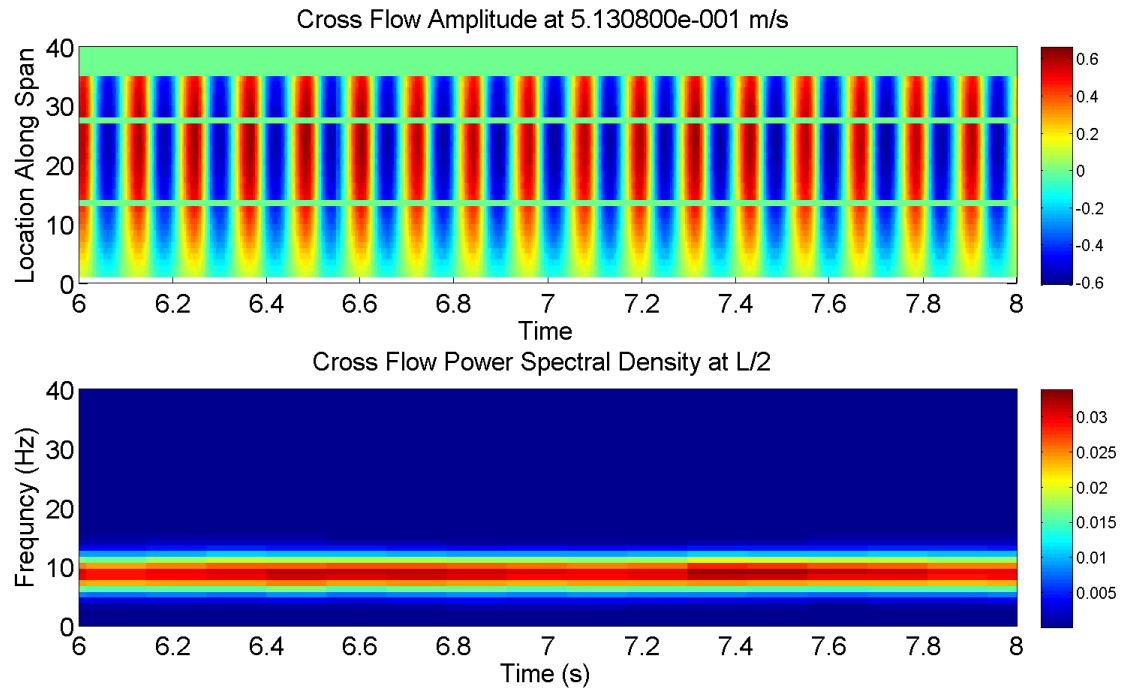

Figure B.6: $\mathrm{Vr}=6.09(\mathrm{U}=0.51 \mathrm{~m} / \mathrm{s})$, Time history of amplitude and PSD of the CF Direction 

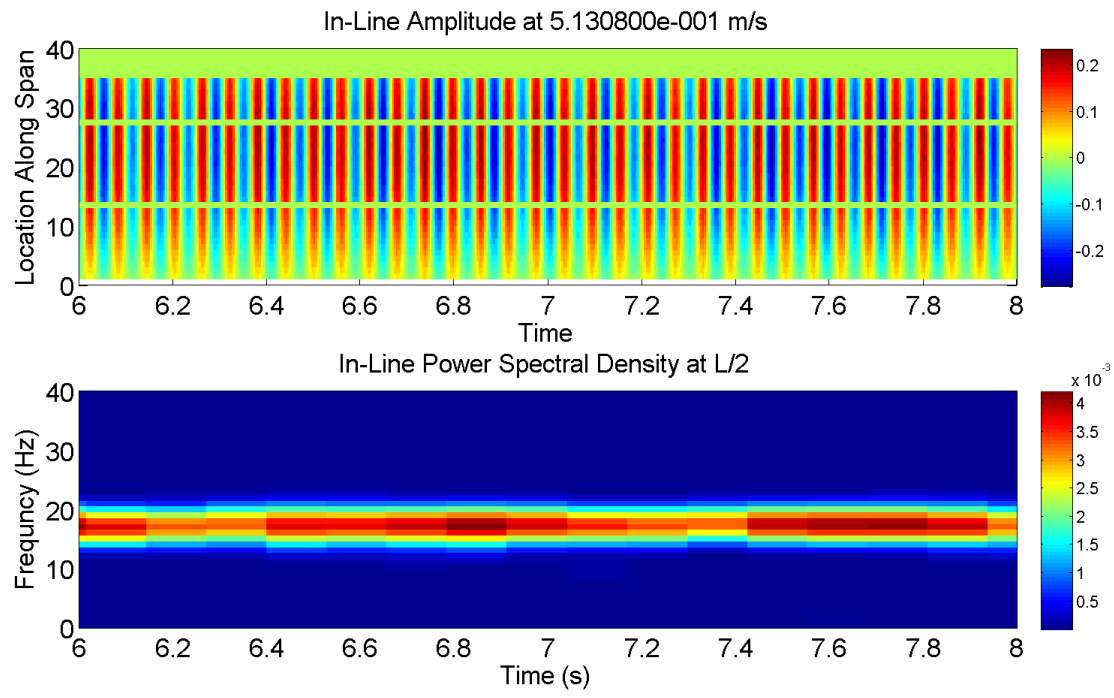

Figure B.7: $\mathrm{Vr}=6.09(\mathrm{U}=0.51 \mathrm{~m} / \mathrm{s})$, Time history of amplitude and PSD of the IL Direction
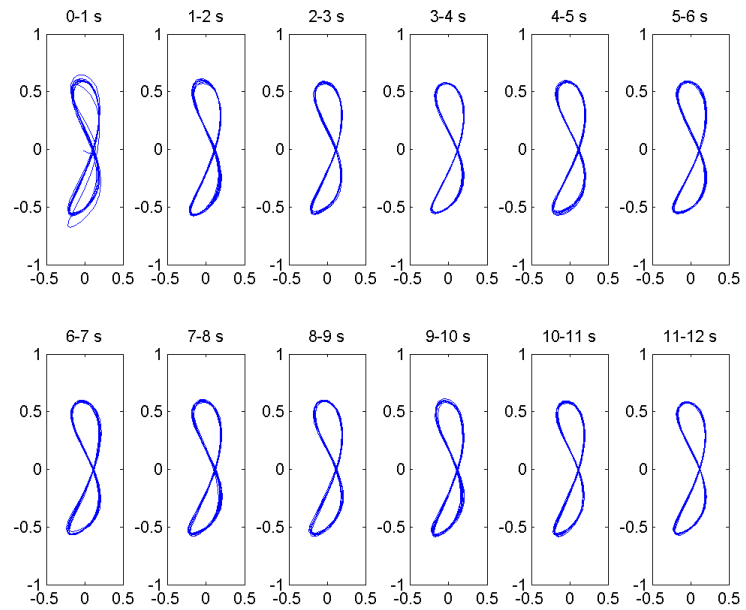

Figure B.8: $\mathrm{Vr}=6.09(\mathrm{U}=0.51 \mathrm{~m} / \mathrm{s})$, orbital patterns $0-12 \mathrm{sec}$ 


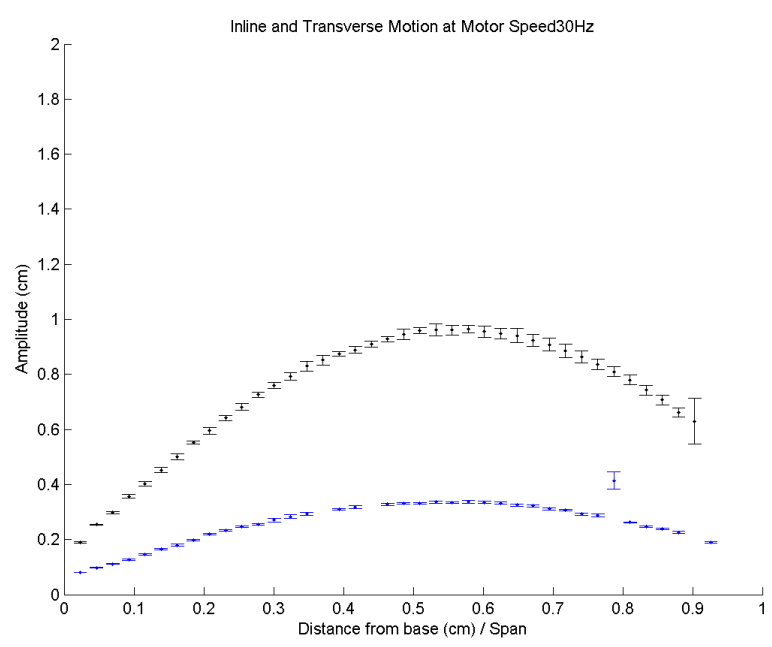

Figure B.9: $\mathrm{Vr}=6.17(\mathrm{U}=0.53 \mathrm{~m} / \mathrm{s})$, Spanwise shape of in-line (Black) and cross-flow (Blue) directions
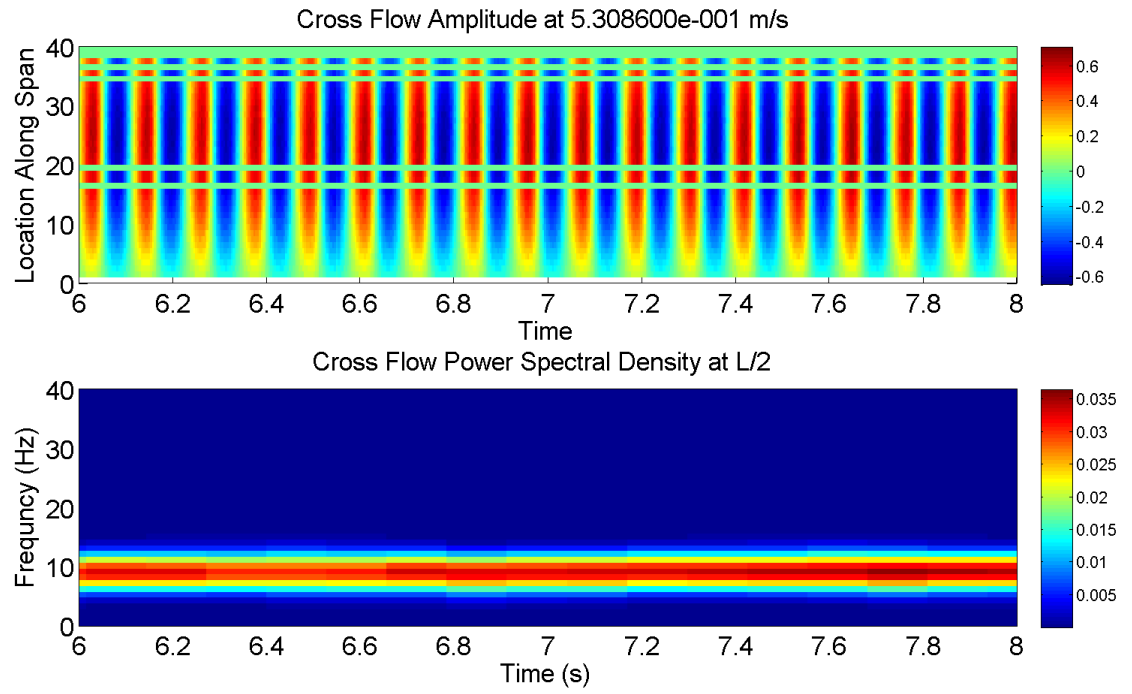

Figure B.10: $\mathrm{Vr}=6.17(\mathrm{U}=0.53 \mathrm{~m} / \mathrm{s})$, Time history of amplitude and PSD of the CF Direction 

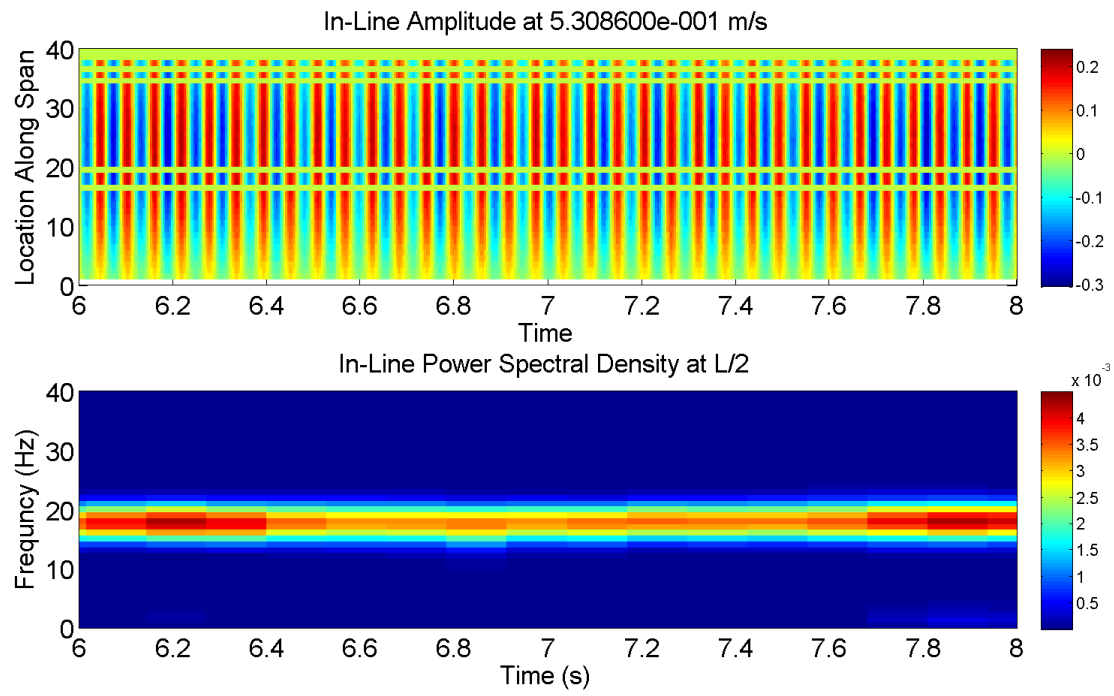

Figure B.11: $\mathrm{Vr}=6.17(\mathrm{U}=0.53 \mathrm{~m} / \mathrm{s})$, Time history of amplitude and PSD of the IL Direction
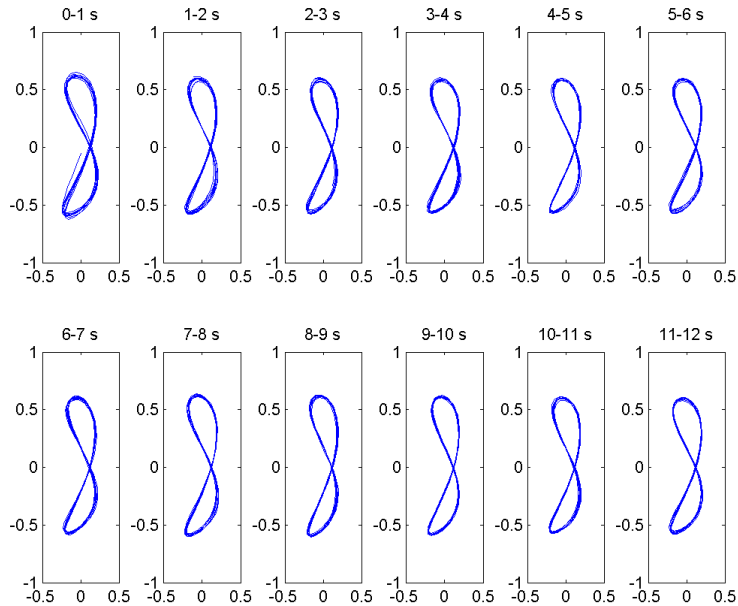

Figure B.12: $\mathrm{Vr}=6.17(\mathrm{U}=0.53 \mathrm{~m} / \mathrm{s})$, orbital patterns $0-12 \mathrm{sec}$ 


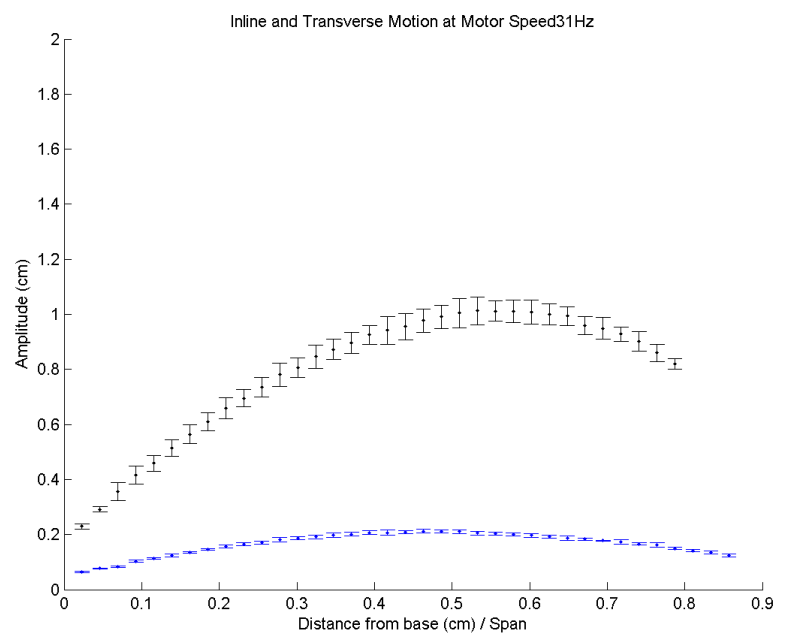

Figure B.13: $\mathrm{Vr}=5.89(\mathrm{U}=0.55 \mathrm{~m} / \mathrm{s})$, Spanwise shape of in-line (Black) and cross-flow (Blue) directions
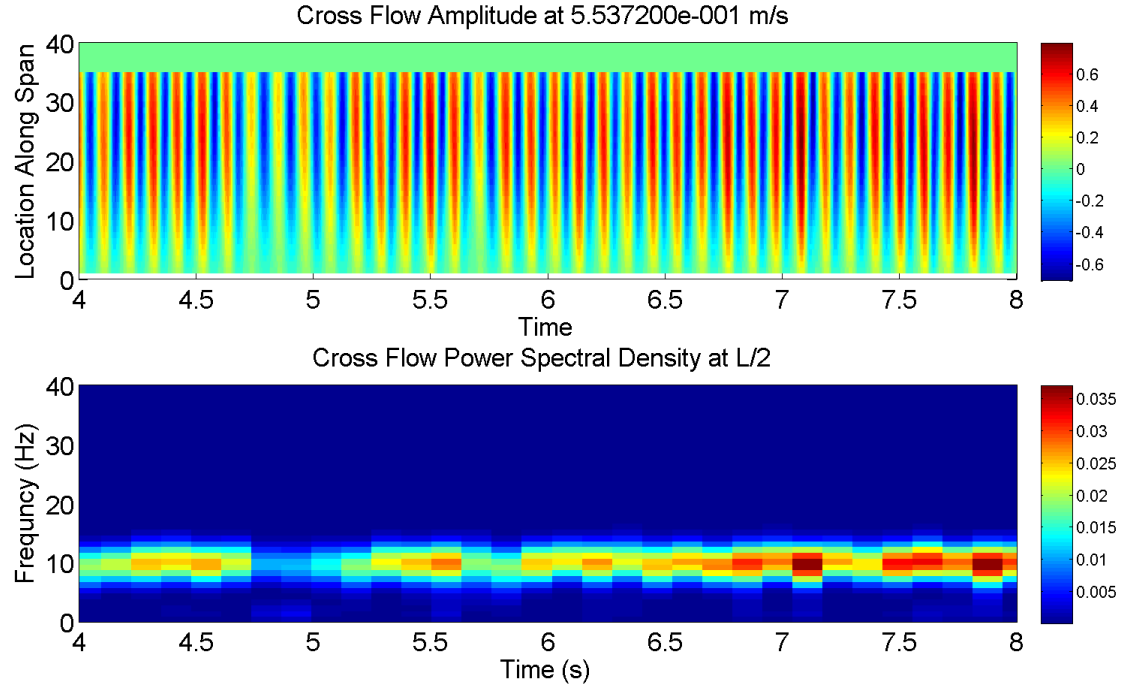

Figure B.14: $\mathrm{Vr}=5.89(\mathrm{U}=0.55 \mathrm{~m} / \mathrm{s})$, Time history of amplitude and PSD of the CF Direction 

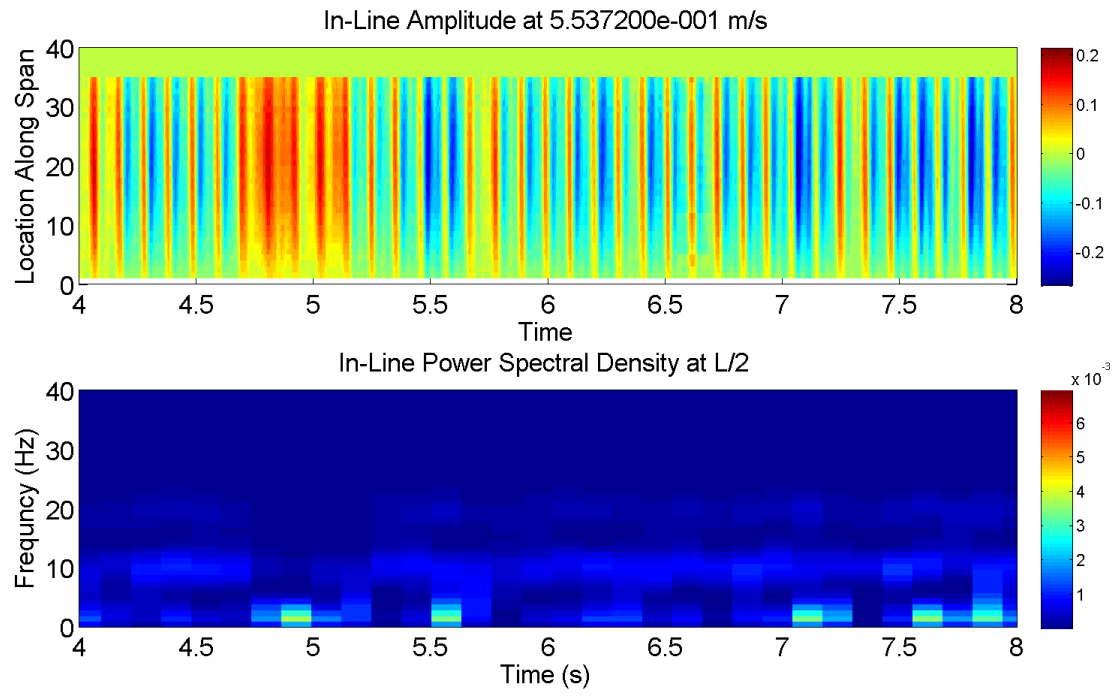

Figure B.15: $\mathrm{Vr}=5.89(\mathrm{U}=0.55 \mathrm{~m} / \mathrm{s})$, Time history of amplitude and PSD of the IL Direction

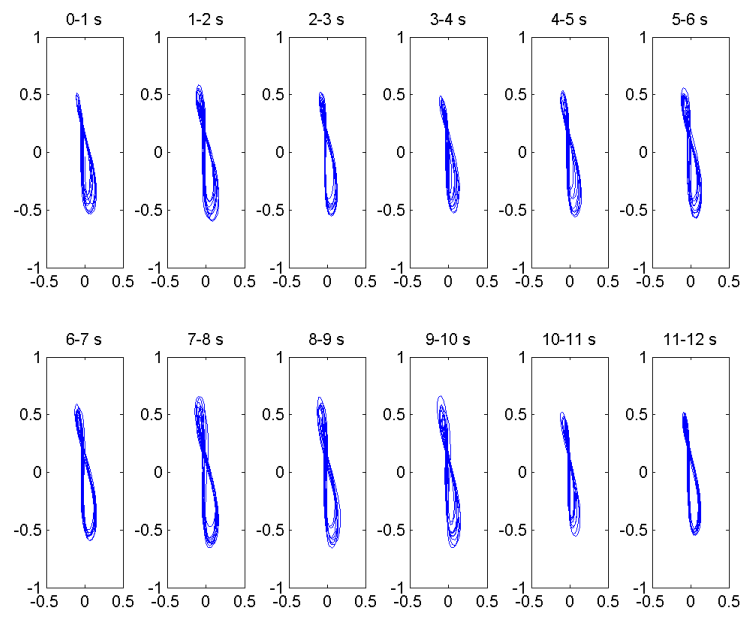

Figure B.16: $\mathrm{Vr}=5.89(\mathrm{U}=0.55 \mathrm{~m} / \mathrm{s})$, orbital patterns $0-12 \mathrm{sec}$ 


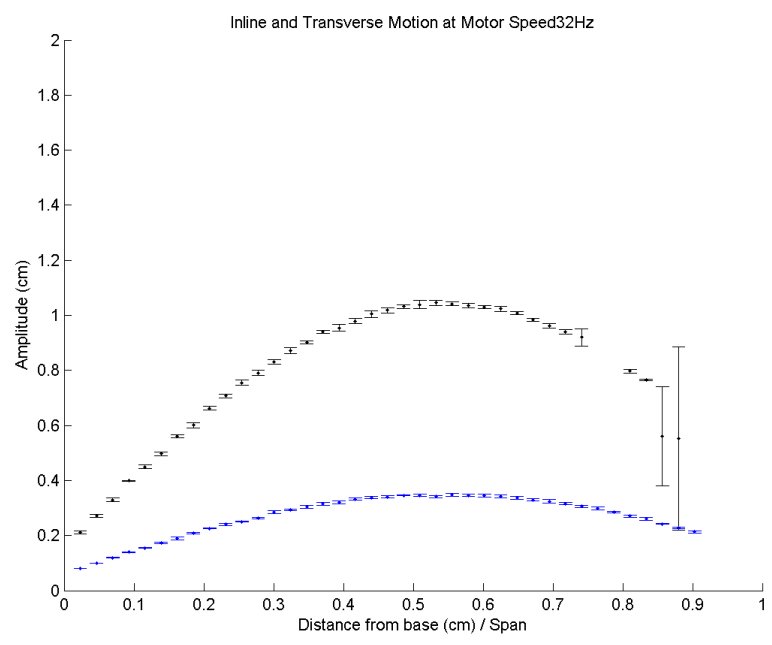

Figure B.17: $\mathrm{Vr}=6.27(\mathrm{U}=0.57 \mathrm{~m} / \mathrm{s})$, Spanwise shape of in-line (Black) and cross-flow (Blue) directions
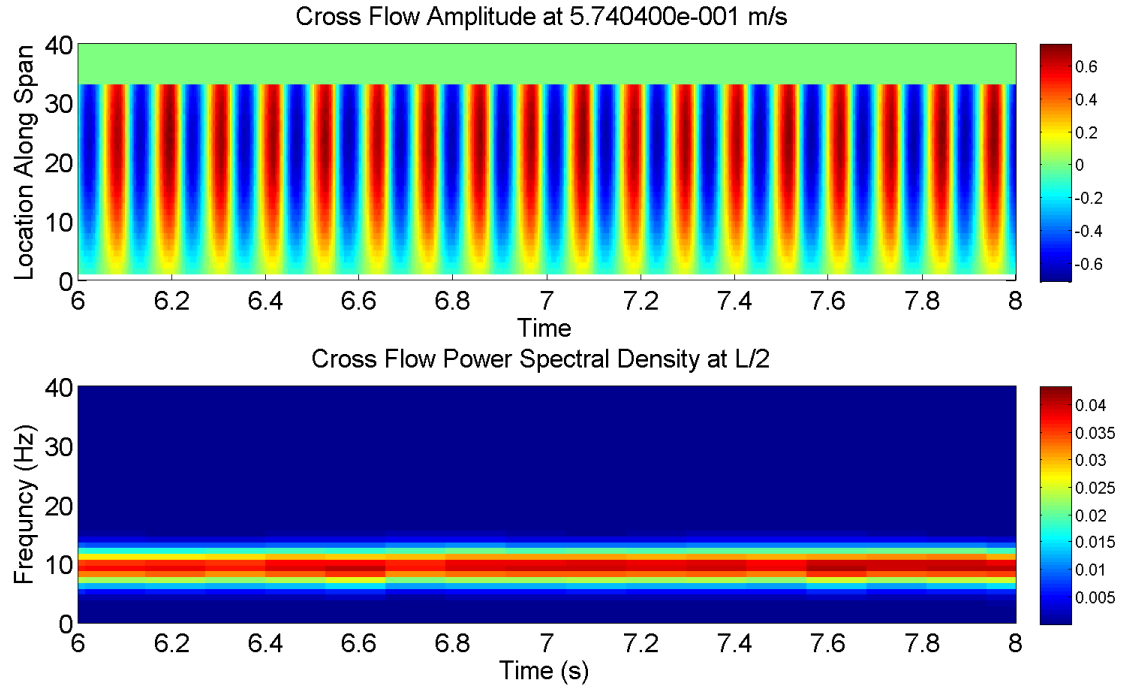

Figure B.18: $\mathrm{Vr}=6.27(\mathrm{U}=0.57 \mathrm{~m} / \mathrm{s})$, Time history of amplitude and PSD of the CF Direction 

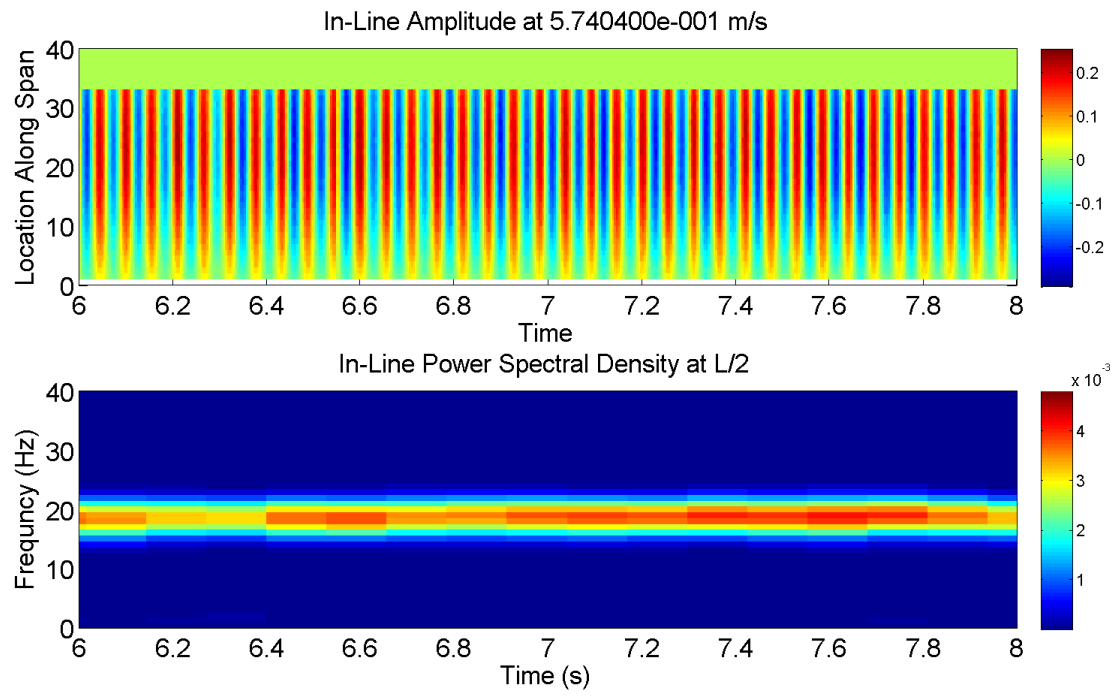

Figure B.19: $\mathrm{Vr}=6.27(\mathrm{U}=0.57 \mathrm{~m} / \mathrm{s})$, Time history of amplitude and PSD of the IL Direction
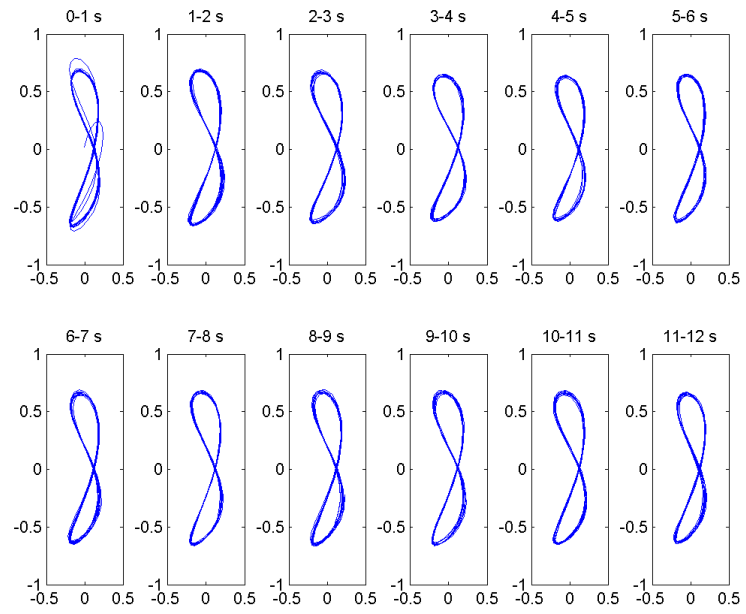

Figure B.20: $\mathrm{Vr}=6.27(\mathrm{U}=0.57 \mathrm{~m} / \mathrm{s})$, orbital patterns $0-12 \mathrm{sec}$ 


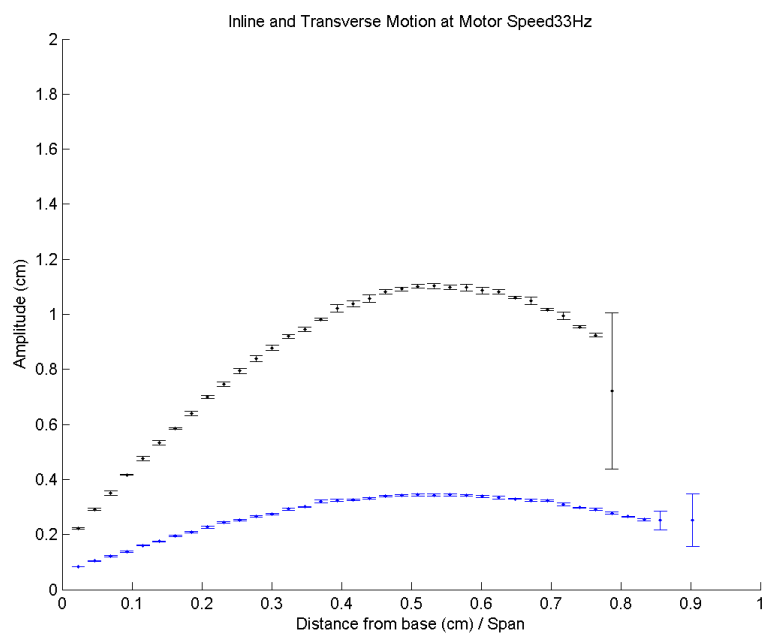

Figure B.21: $\mathrm{Vr}=6.30(\mathrm{U}=0.58 \mathrm{~m} / \mathrm{s})$, Spanwise shape of in-line (Black) and cross-flow (Blue) directions
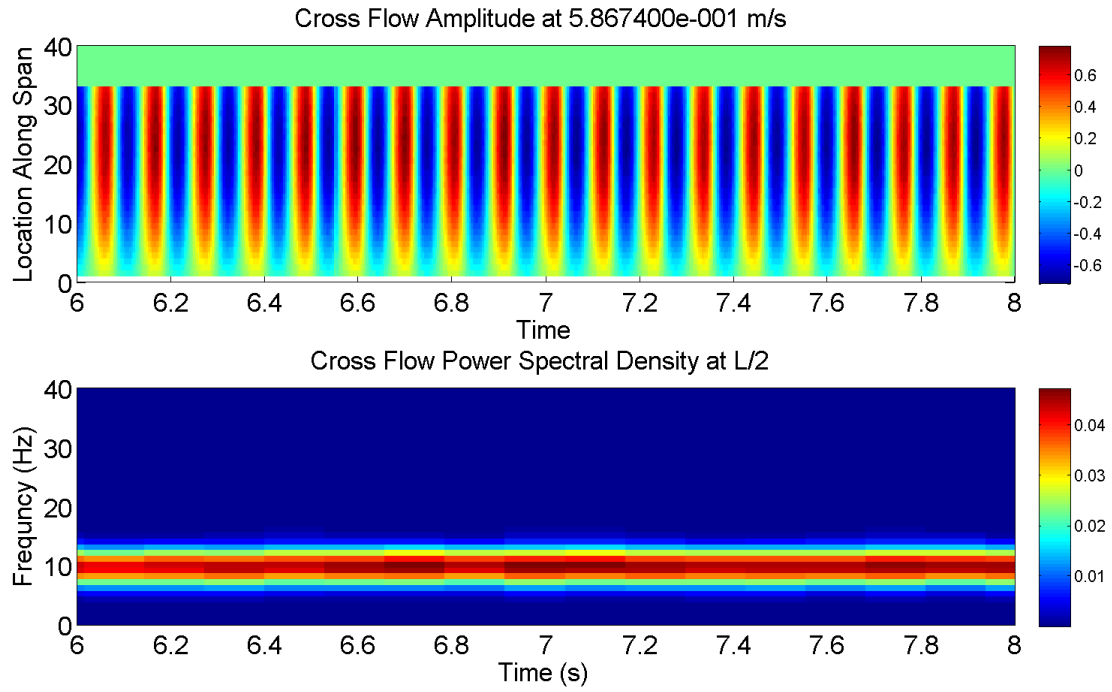

Figure B.22: $\mathrm{Vr}=6.30(\mathrm{U}=0.58 \mathrm{~m} / \mathrm{s})$, Time history of amplitude and PSD of the CF Direction 

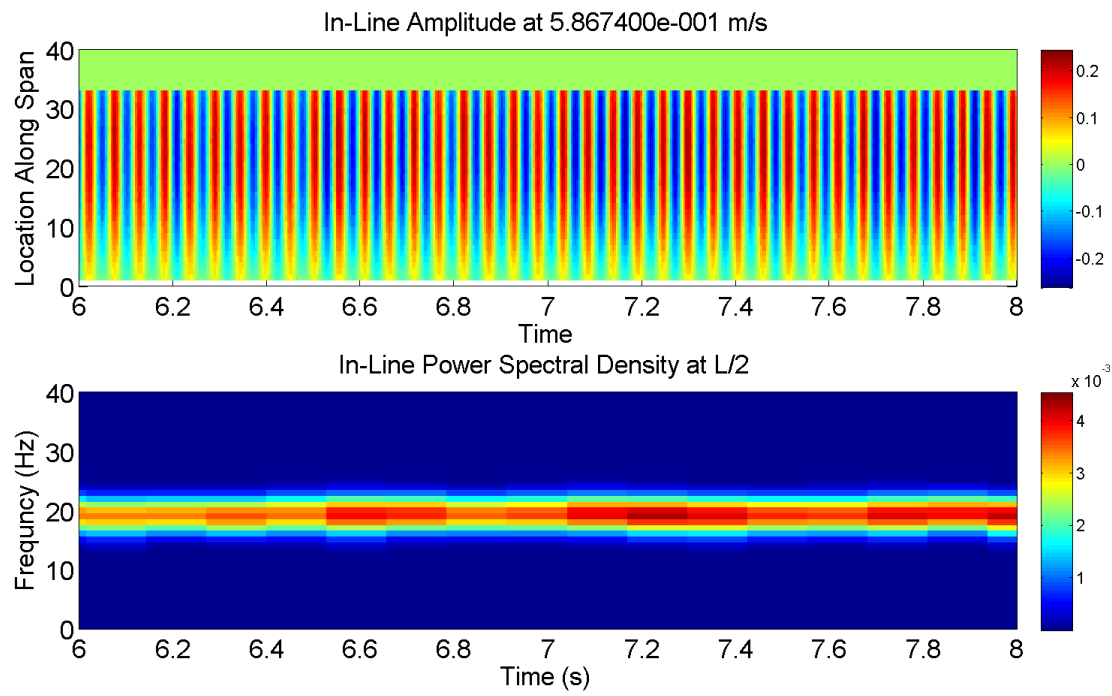

Figure B.23: $\mathrm{Vr}=6.30(\mathrm{U}=0.58 \mathrm{~m} / \mathrm{s})$, Time history of amplitude and PSD of the IL Direction
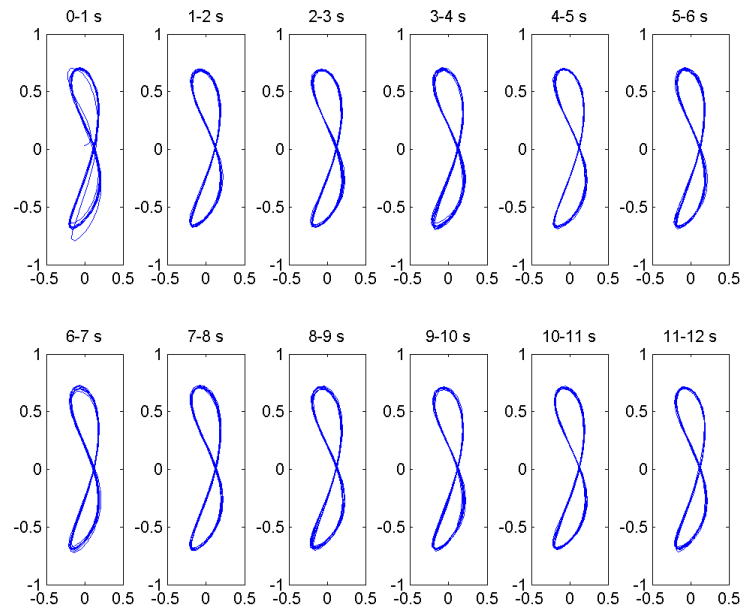

Figure B.24: $\mathrm{Vr}=6.30(\mathrm{U}=0.58 \mathrm{~m} / \mathrm{s})$, orbital patterns $0-12 \mathrm{sec}$ 


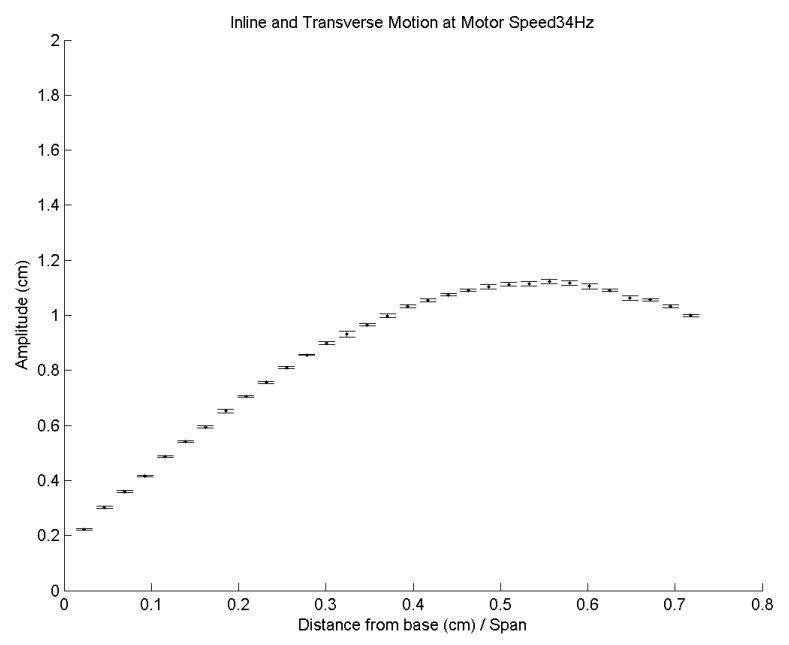

Figure B.25: $\mathrm{Vr}=6.36(\mathrm{U}=0.61 \mathrm{~m} / \mathrm{s})$, Spanwise shape of in-line (Black) and cross-flow (Blue) directions
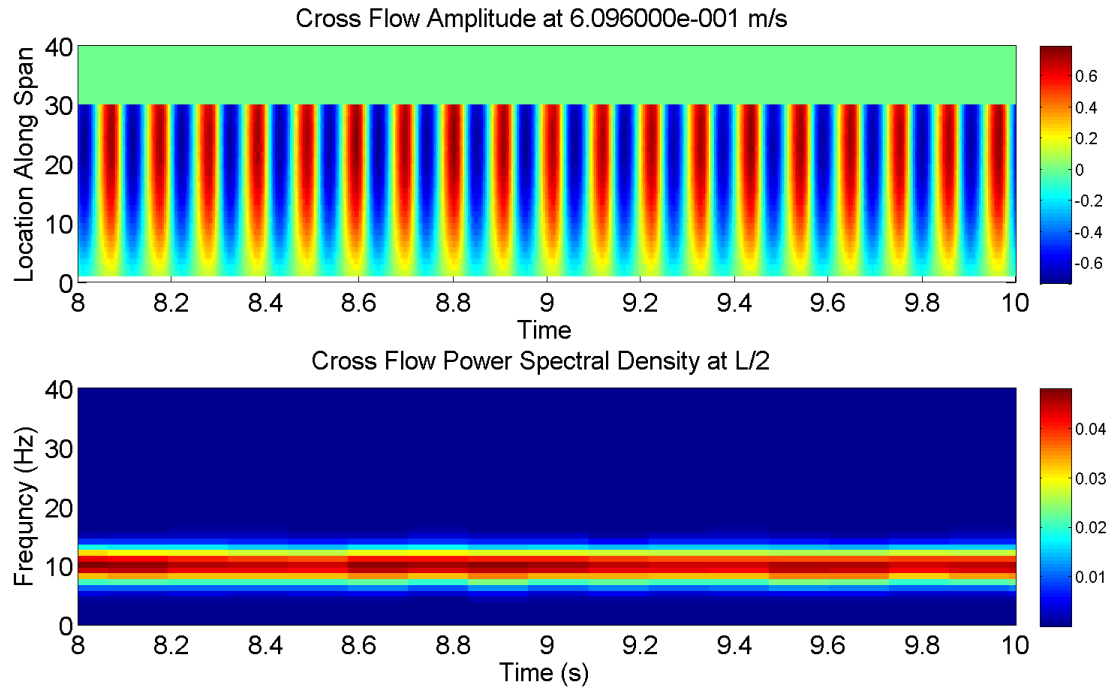

Figure B.26: $\mathrm{Vr}=6.36(\mathrm{U}=0.61 \mathrm{~m} / \mathrm{s})$, Time history of amplitude and PSD of the CF Direction 

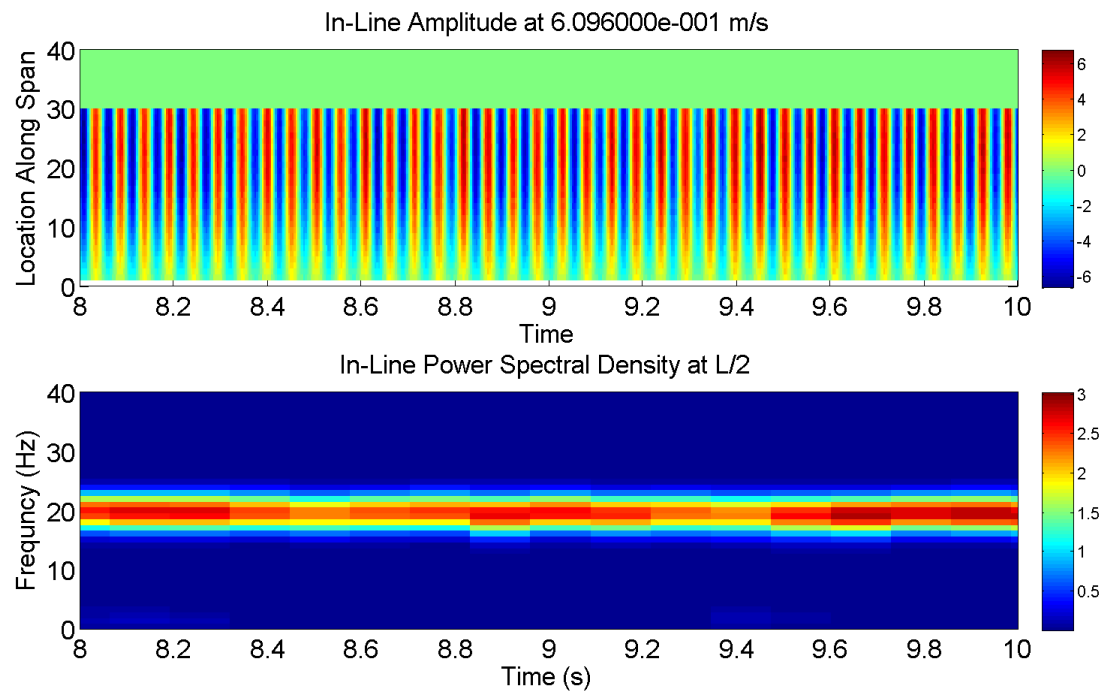

Figure B.27: $\mathrm{Vr}=6.36(\mathrm{U}=0.61 \mathrm{~m} / \mathrm{s})$, Time history of amplitude and PSD of the IL Direction
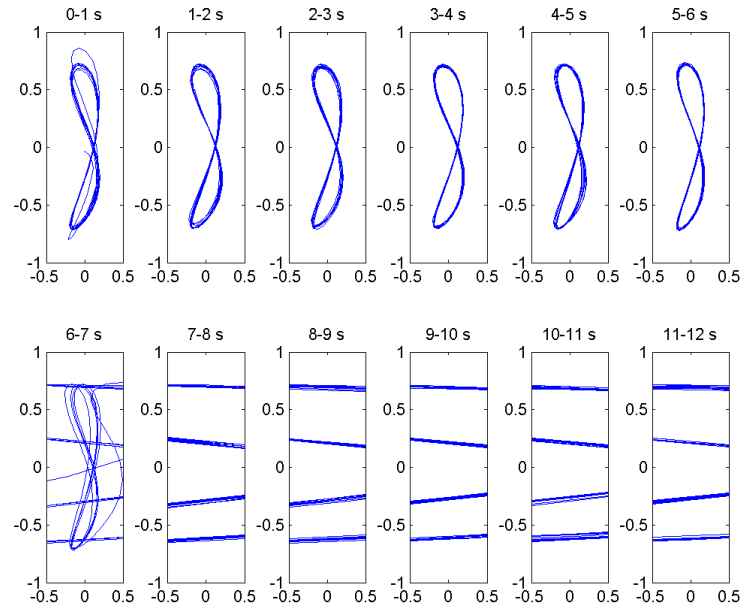

Figure B.28: $\mathrm{Vr}=6.36(\mathrm{U}=0.61 \mathrm{~m} / \mathrm{s})$, orbital patterns $0-12$ sec. At 6.5 seconds point tracking was lost during data analysis 


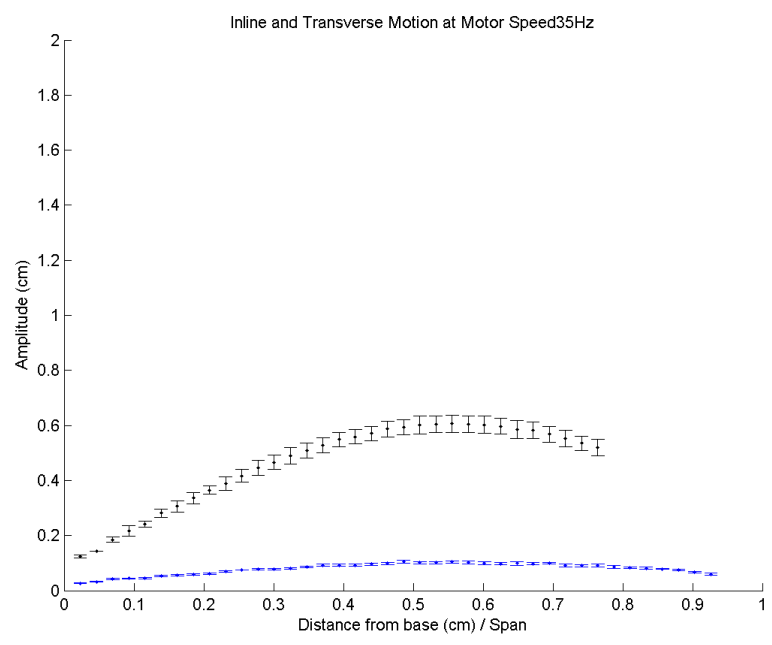

Figure B.29: $\mathrm{Vr}=5.68(\mathrm{U}=0.624 \mathrm{~m} / \mathrm{s})$, Spanwise shape of in-line (Black) and cross-flow (Blue) directions
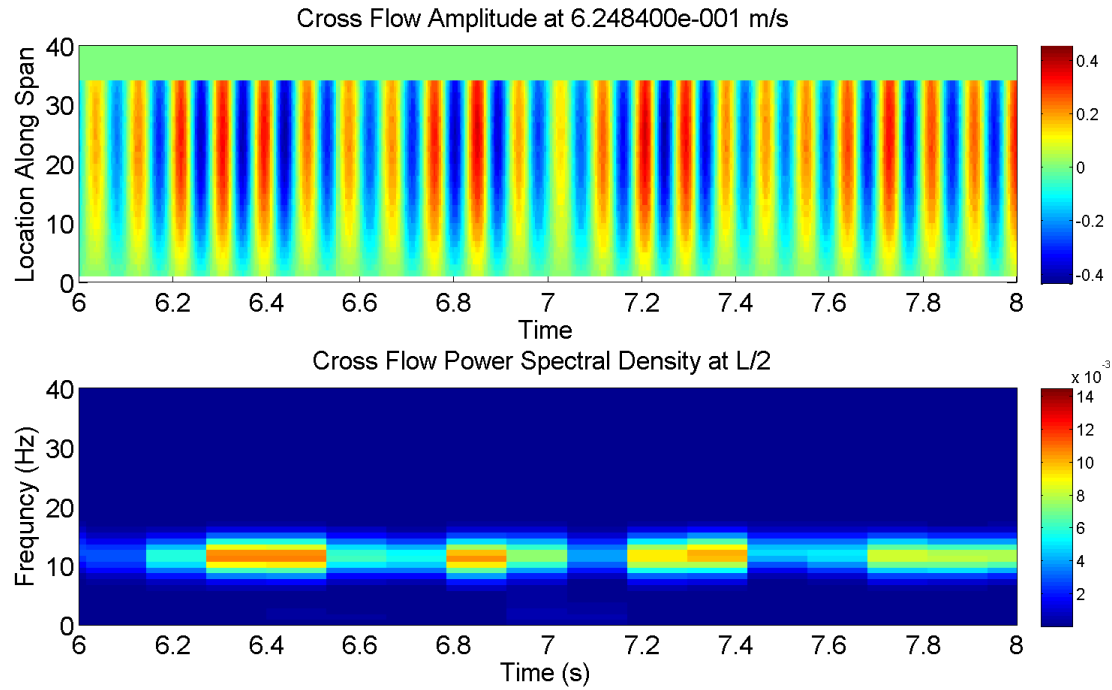

Figure B.30: $\mathrm{Vr}=5.68(\mathrm{U}=0.624 \mathrm{~m} / \mathrm{s})$, Time history of amplitude and PSD of the CF Direction 

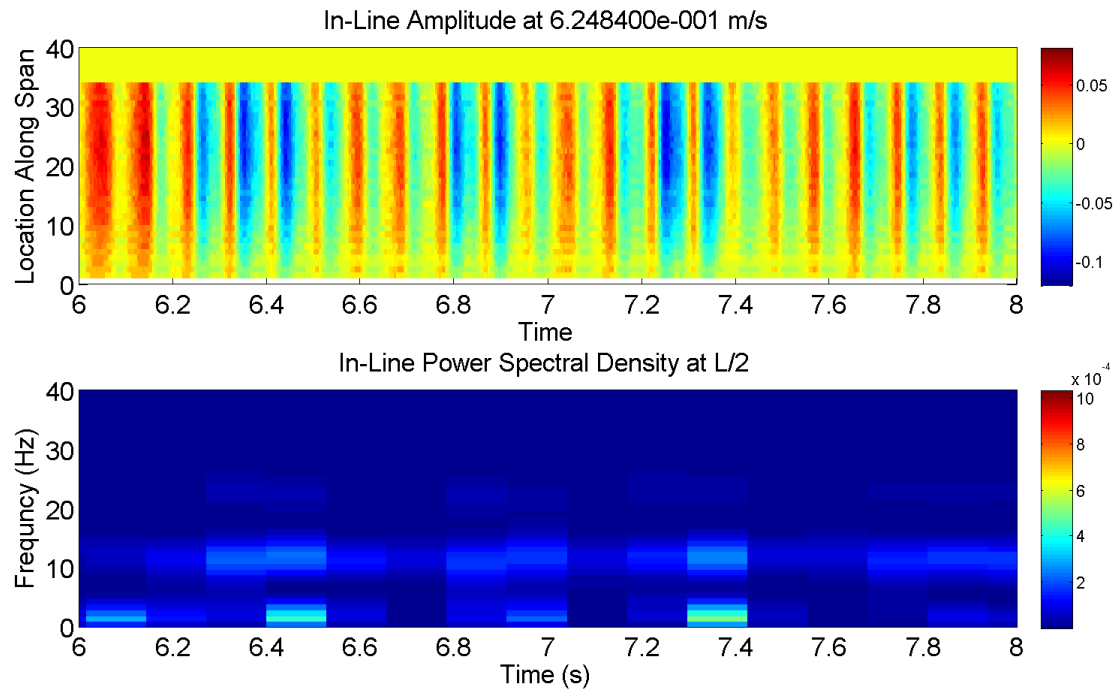

Figure B.31: $\mathrm{Vr}=5.68(\mathrm{U}=0.624 \mathrm{~m} / \mathrm{s})$, Time history of amplitude and PSD of the IL Direction
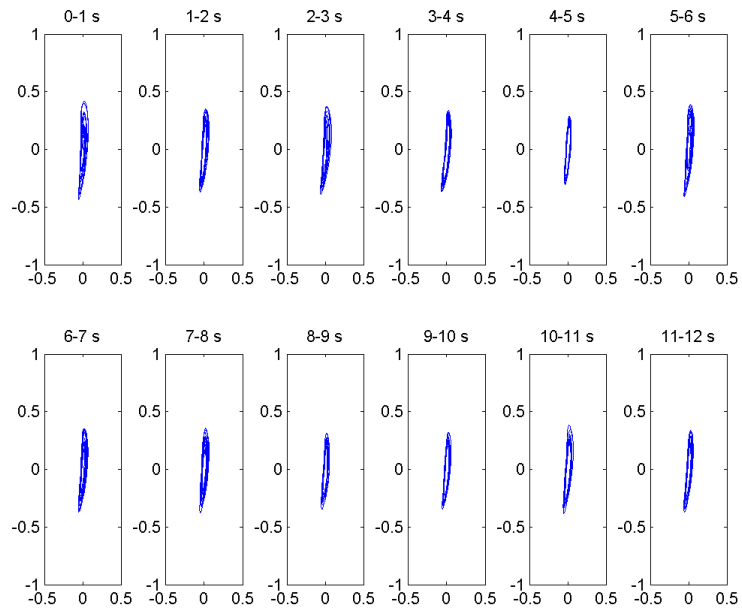

Figure B.32: $\mathrm{Vr}=5.68(\mathrm{U}=0.624 \mathrm{~m} / \mathrm{s})$, orbital patterns $0-12 \mathrm{sec}$ 


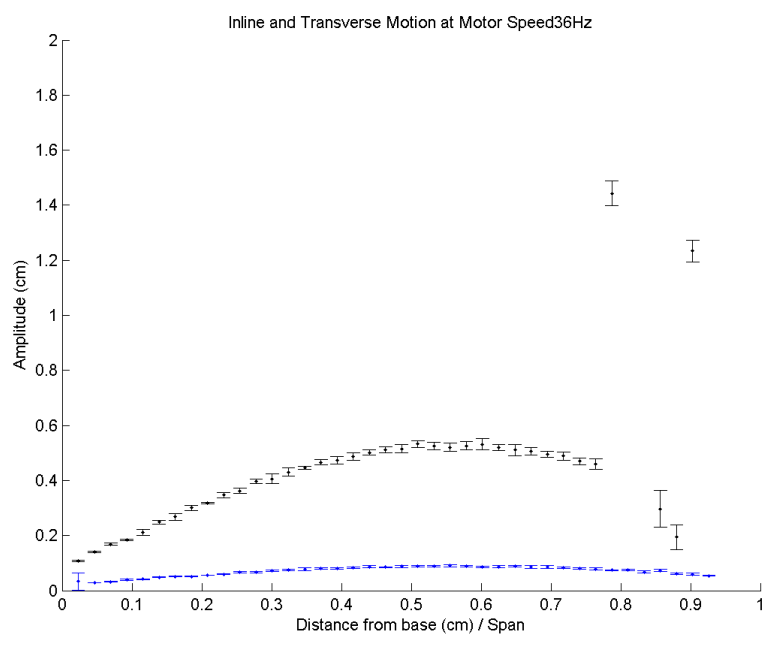

Figure B.33: $\mathrm{Vr}=5.73(\mathrm{U}=0.647 \mathrm{~m} / \mathrm{s})$, Spanwise shape of in-line (Black) and cross-flow (Blue) directions
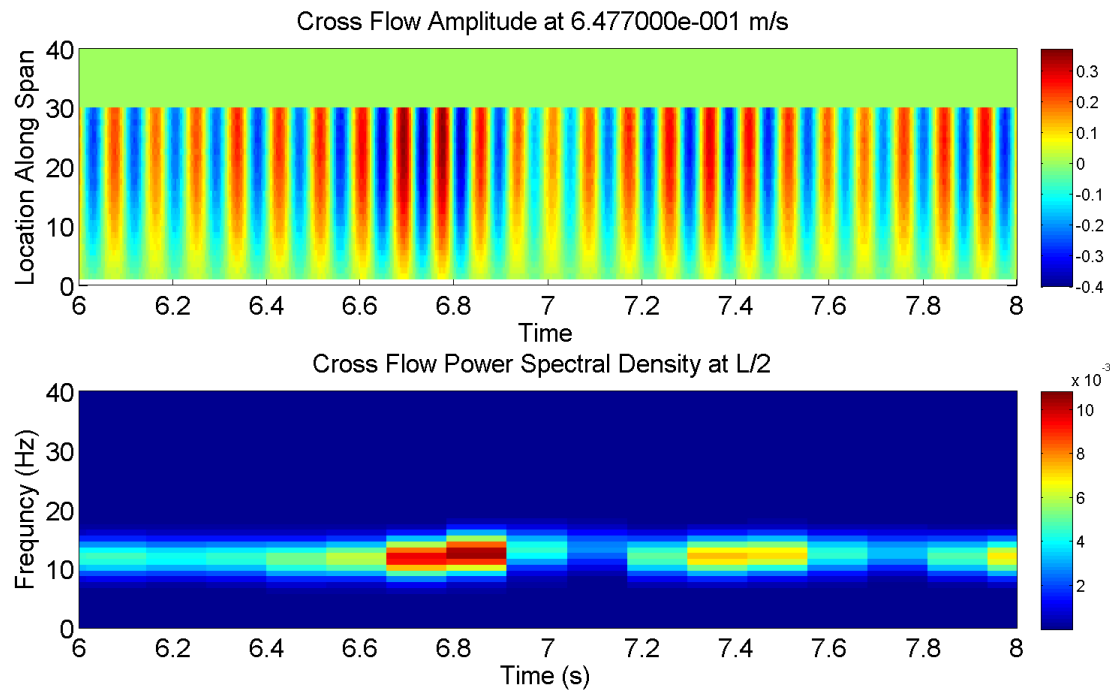

Figure B.34: $\mathrm{Vr}=5.73(\mathrm{U}=0.647 \mathrm{~m} / \mathrm{s})$, Time history of amplitude and PSD of the CF Direction 

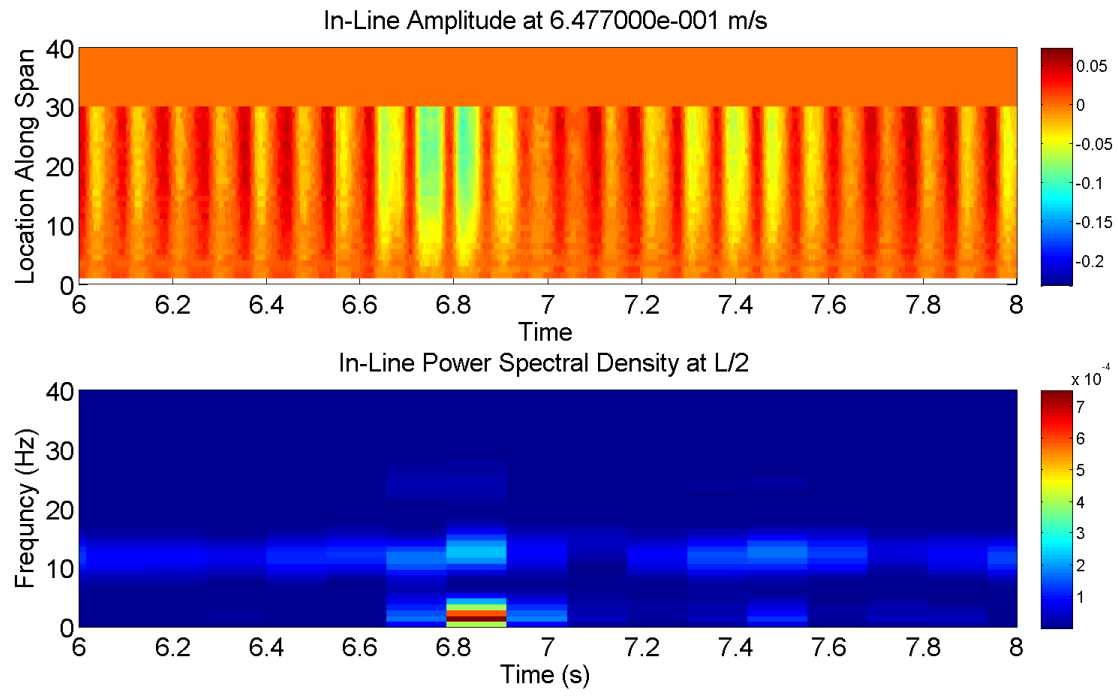

Figure B.35: $\mathrm{Vr}=5.73(\mathrm{U}=0.647 \mathrm{~m} / \mathrm{s})$, Time history of amplitude and PSD of the IL Direction
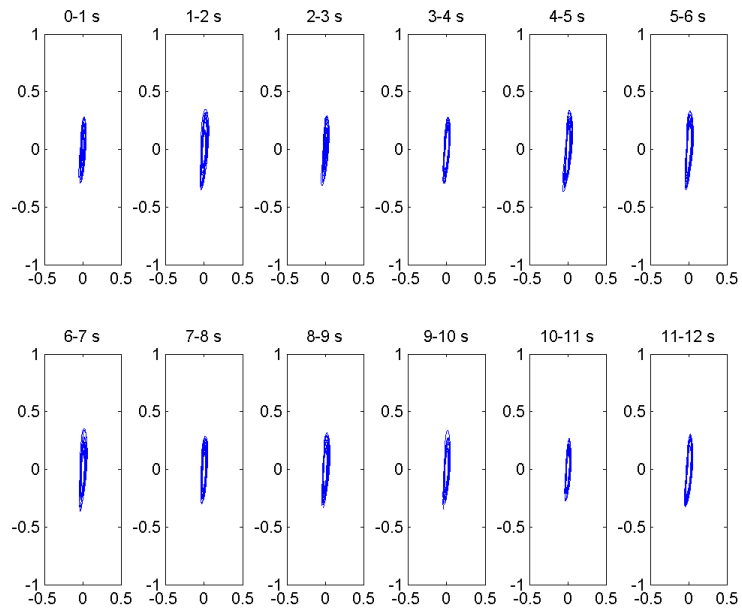

Figure B.36: $\mathrm{Vr}=5.73(\mathrm{U}=0.647 \mathrm{~m} / \mathrm{s})$, orbital patterns $0-12 \mathrm{sec}$ 


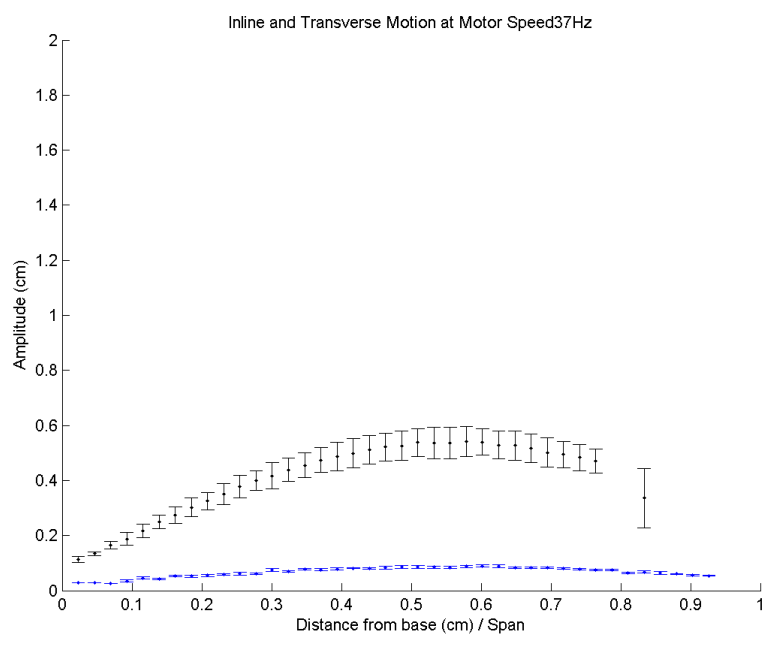

Figure B.37: $\mathrm{Vr}=5.65(\mathrm{U}=0.66 \mathrm{~m} / \mathrm{s})$, Spanwise shape of in-line (Black) and cross-flow (Blue) directions
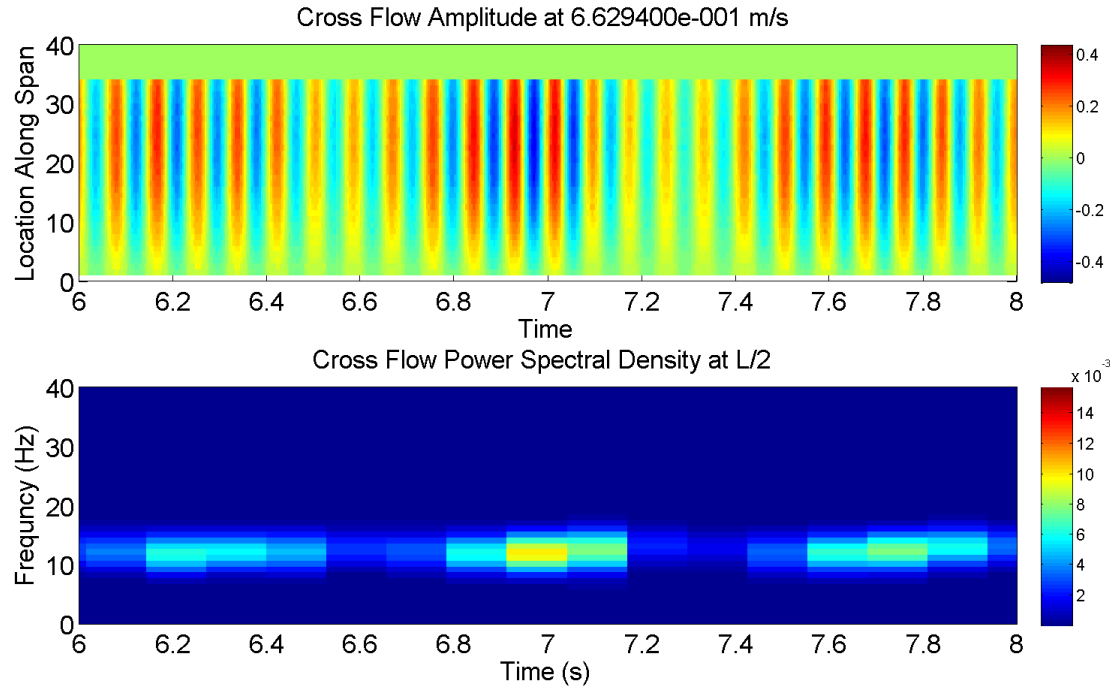

Figure B.38: $\mathrm{Vr}=5.65(\mathrm{U}=0.66 \mathrm{~m} / \mathrm{s})$, Time history of amplitude and PSD of the CF Direction 

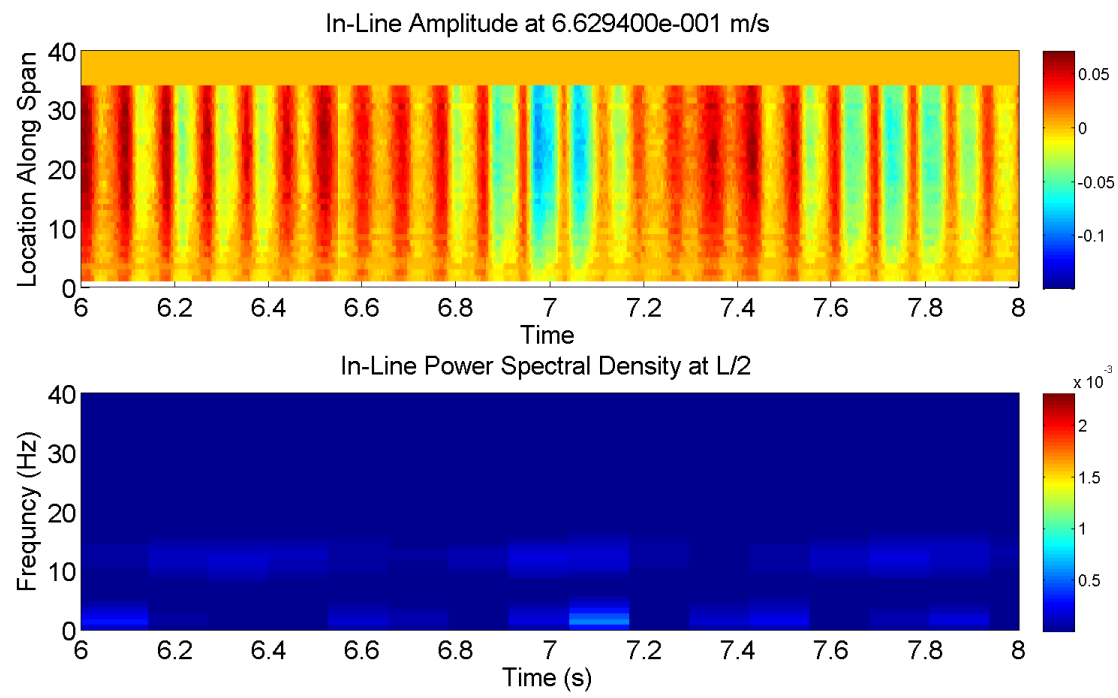

Figure B.39: $\mathrm{Vr}=5.65(\mathrm{U}=0.66 \mathrm{~m} / \mathrm{s})$, Time history of amplitude and PSD of the IL Direction
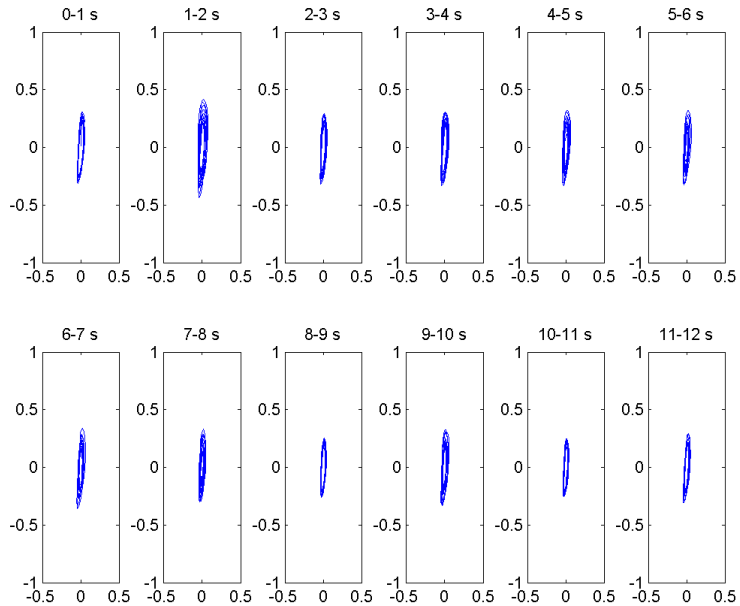

Figure B.40: $\mathrm{Vr}=5.65(\mathrm{U}=0.66 \mathrm{~m} / \mathrm{s})$, orbital patterns $0-12 \mathrm{sec}$ 


\section{APPENDIX $\mathrm{C}$}

\section{Appendix C}

Second Mode In-line and First Mode Cross-Flow (2-1 Cylinder Results)

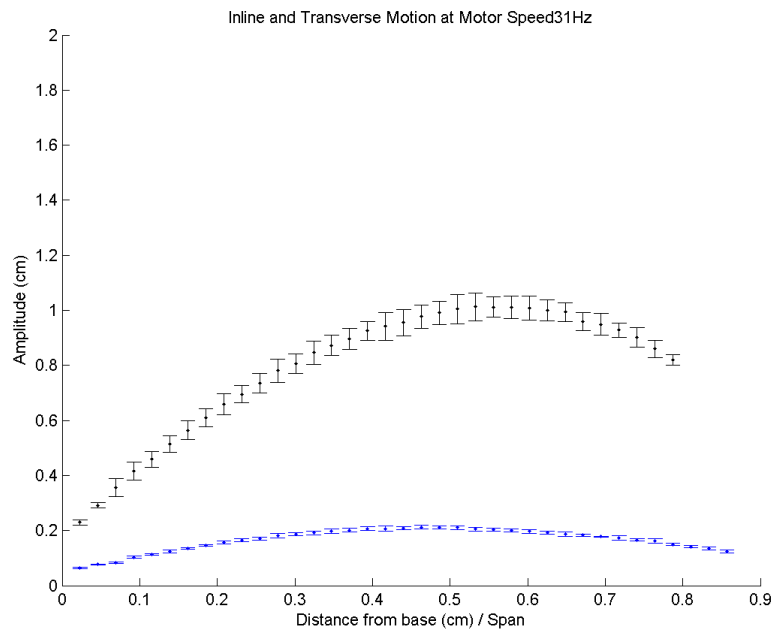

Figure C.1: $\mathrm{Vr}=5.89(\mathrm{U}=0.55 \mathrm{~m} / \mathrm{s})$, Spanwise shape of in-line (Black) and cross-flow (Blue) directions
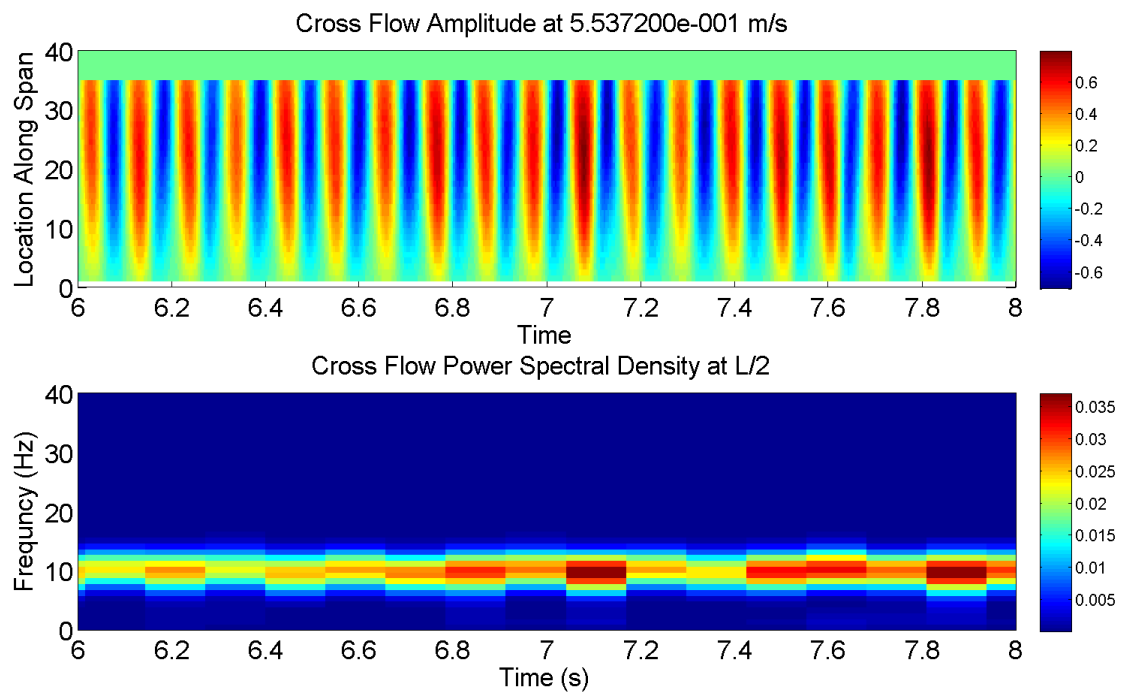

Figure C.2: $\mathrm{Vr}=5.89(\mathrm{U}=0.55 \mathrm{~m} / \mathrm{s})$, Time history of amplitude and PSD of the CF Direction 

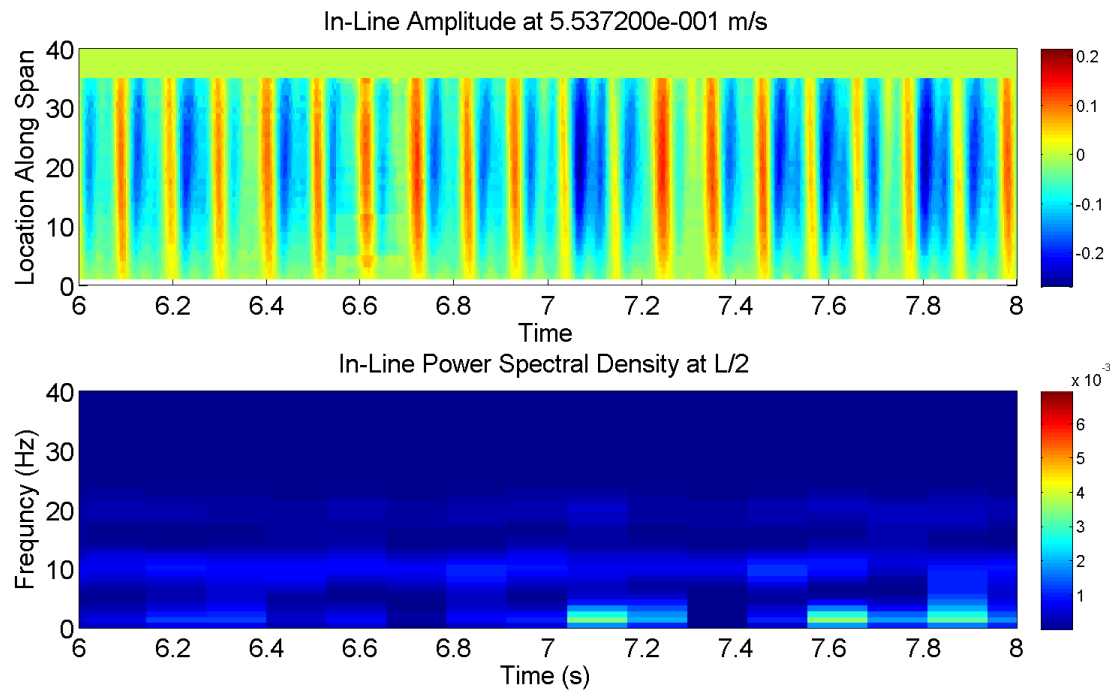

Figure C.3: $\mathrm{Vr}=5.89(\mathrm{U}=0.55 \mathrm{~m} / \mathrm{s})$, Time history of amplitude and PSD of the IL Direction

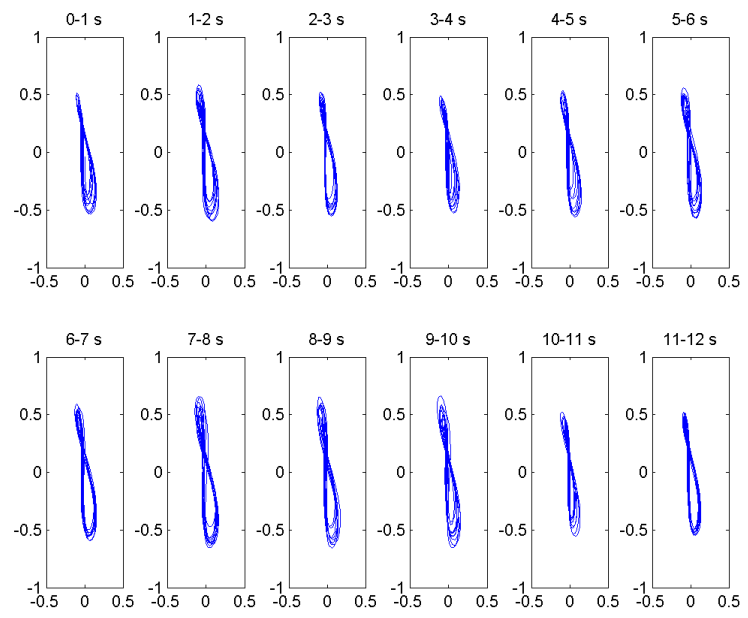

Figure C.4: $\mathrm{Vr}=5.89(\mathrm{U}=0.55 \mathrm{~m} / \mathrm{s})$, orbital patterns $0-12 \mathrm{sec}$ 


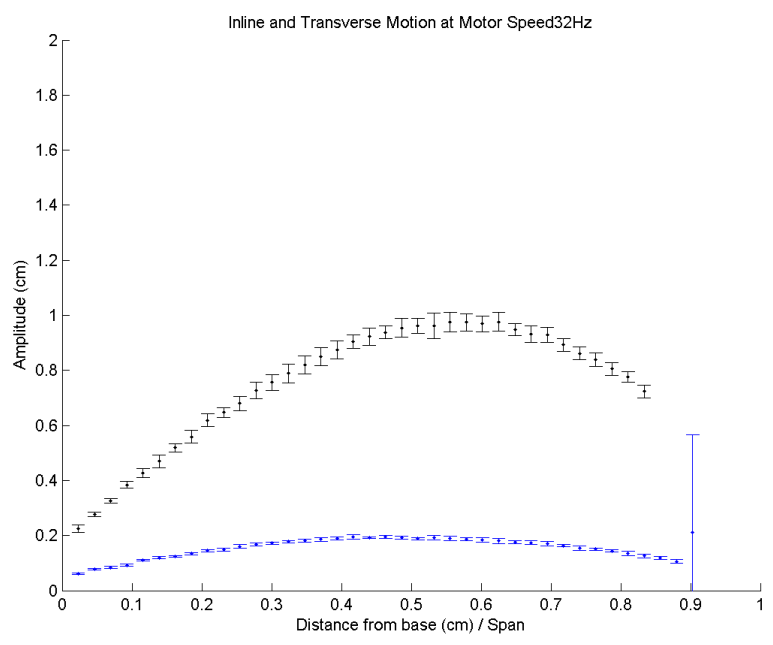

Figure C.5: $\mathrm{Vr}=6.03(\mathrm{U}=0.57 \mathrm{~m} / \mathrm{s})$, Spanwise shape of in-line (Black) and cross-flow (Blue) directions
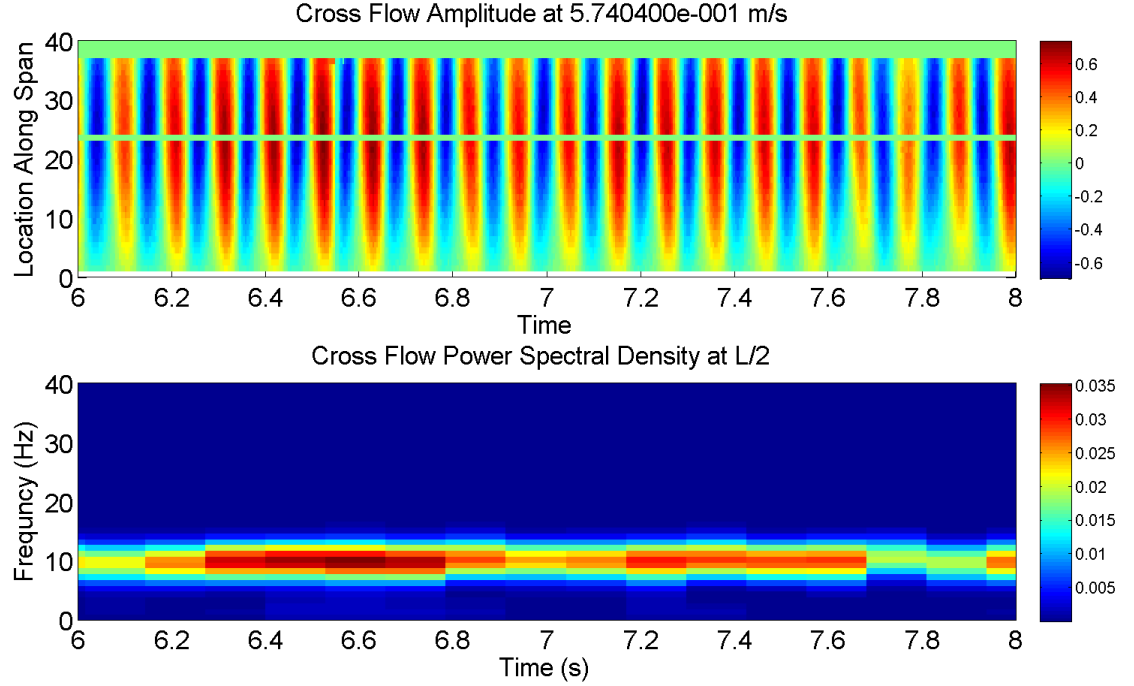

Figure C.6: $\mathrm{Vr}=6.03(\mathrm{U}=0.57 \mathrm{~m} / \mathrm{s})$, Time history of amplitude and PSD of the CF Direction 

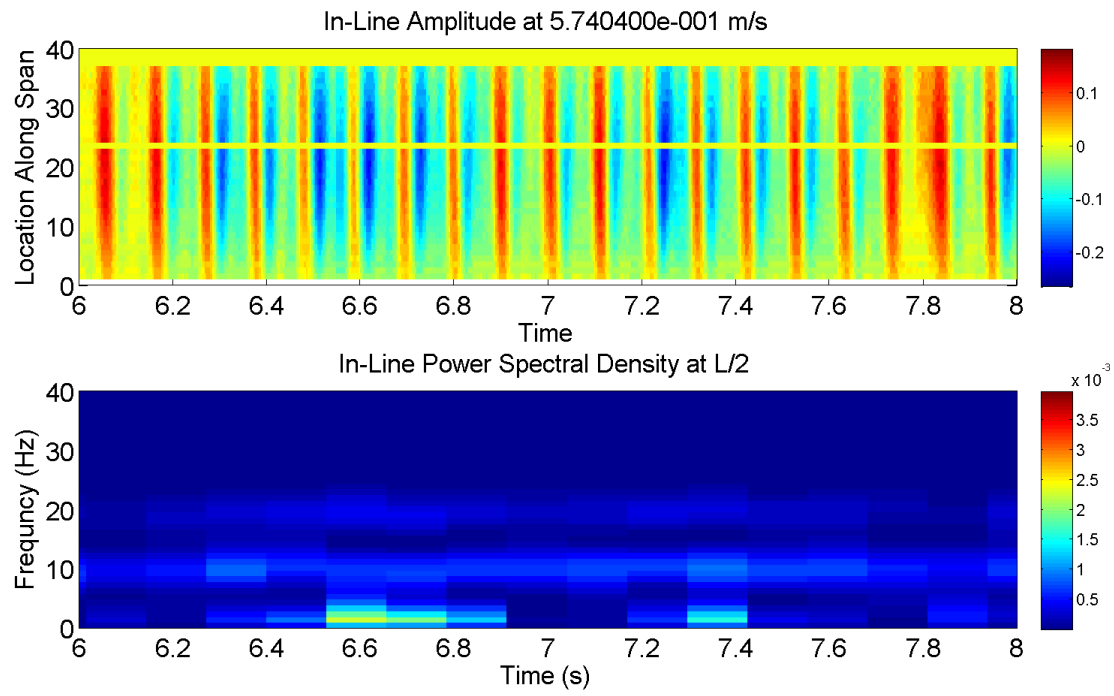

Figure C.7: $\mathrm{Vr}=6.03(\mathrm{U}=0.57 \mathrm{~m} / \mathrm{s})$, Time history of amplitude and PSD of the IL Direction

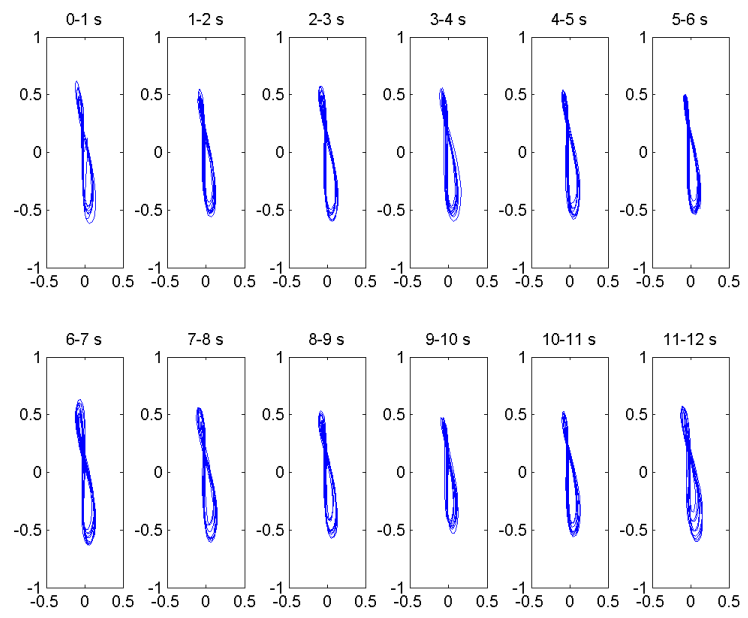

Figure C.8: $\mathrm{Vr}=6.03(\mathrm{U}=0.57 \mathrm{~m} / \mathrm{s})$, orbital patterns $0-12 \mathrm{sec}$ 


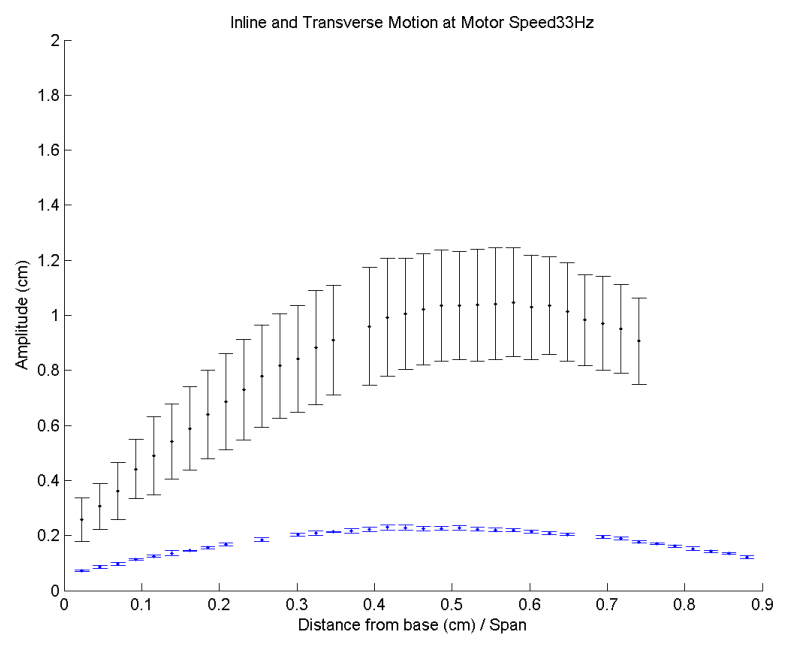

Figure C.9: $\mathrm{Vr}=6.00(\mathrm{U}=0.59 \mathrm{~m} / \mathrm{s})$, Spanwise shape of in-line (Black) and cross-flow (Blue) directions
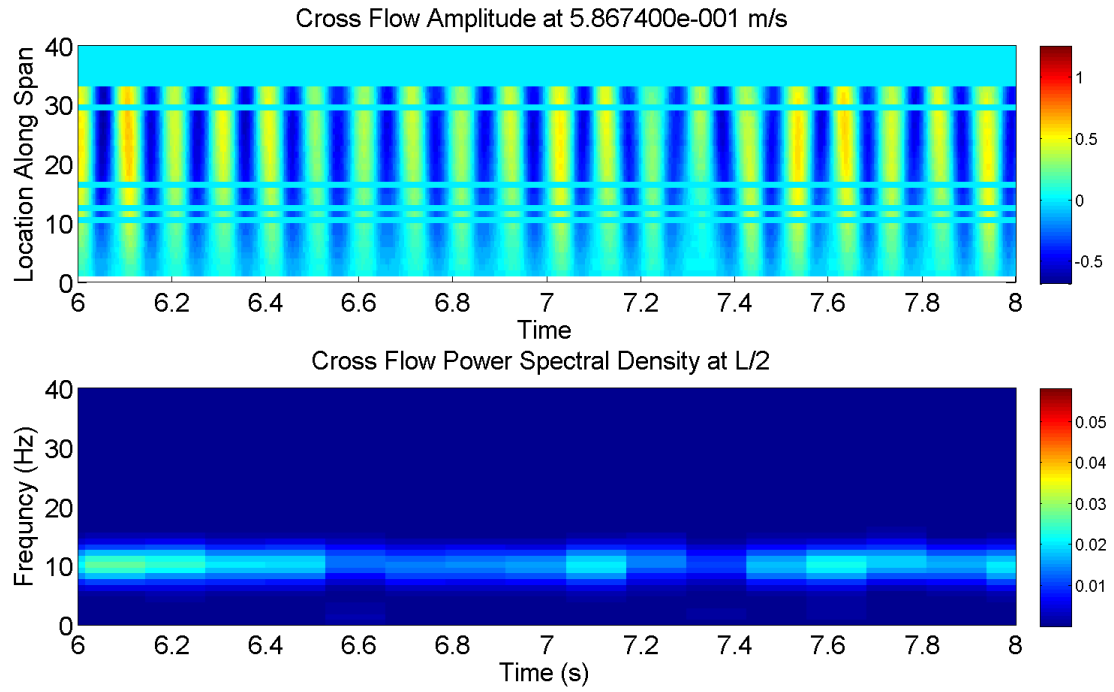

Figure C.10: $\mathrm{Vr}=6.00(\mathrm{U}=0.59 \mathrm{~m} / \mathrm{s})$, Time history of amplitude and PSD of the CF Direction 

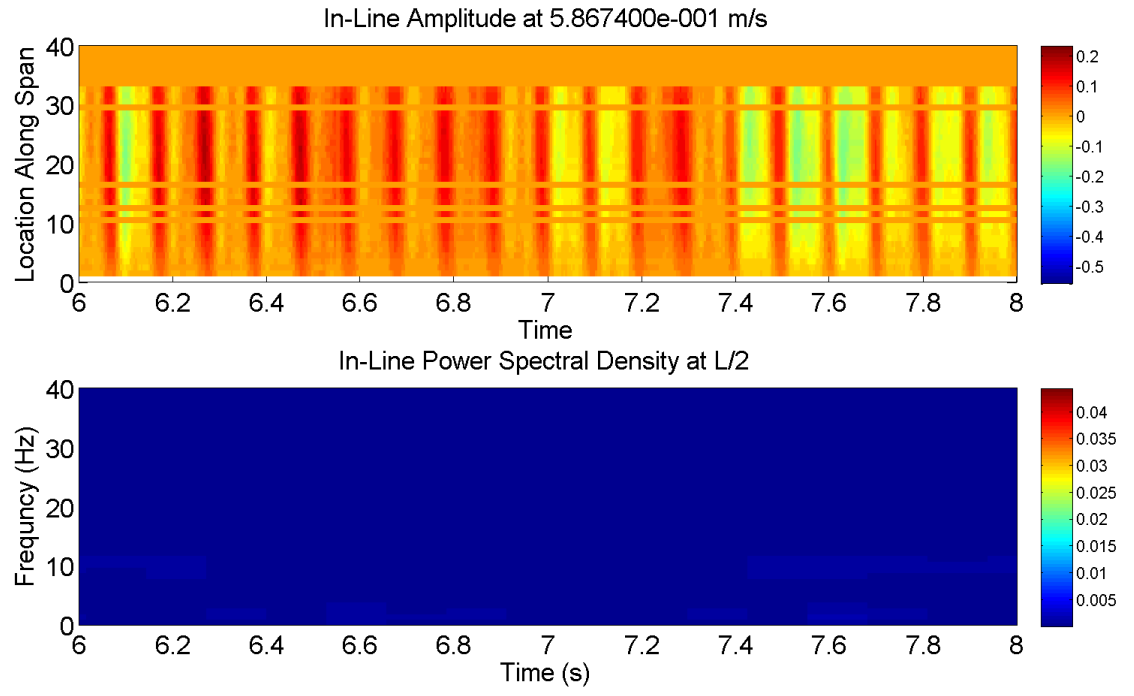

Figure C.11: $\mathrm{Vr}=6.00(\mathrm{U}=0.59 \mathrm{~m} / \mathrm{s})$, Time history of amplitude and PSD of the IL Direction

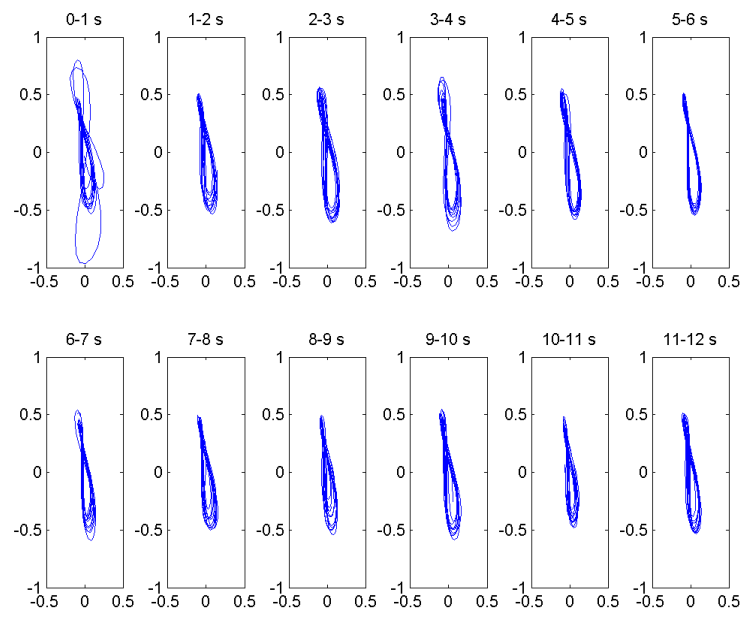

Figure C.12: $\mathrm{Vr}=6.00(\mathrm{U}=0.59 \mathrm{~m} / \mathrm{s})$, orbital patterns $0-12 \mathrm{sec}$ 


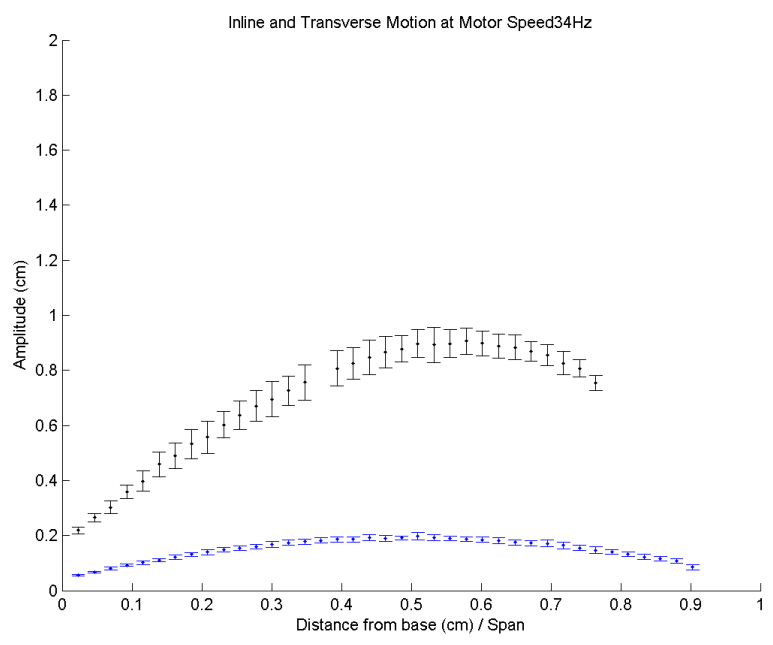

Figure C.13: $\mathrm{Vr}=6.127(\mathrm{U}=0.61 \mathrm{~m} / \mathrm{s})$, Spanwise shape of in-line (Black) and cross-flow (Blue) directions
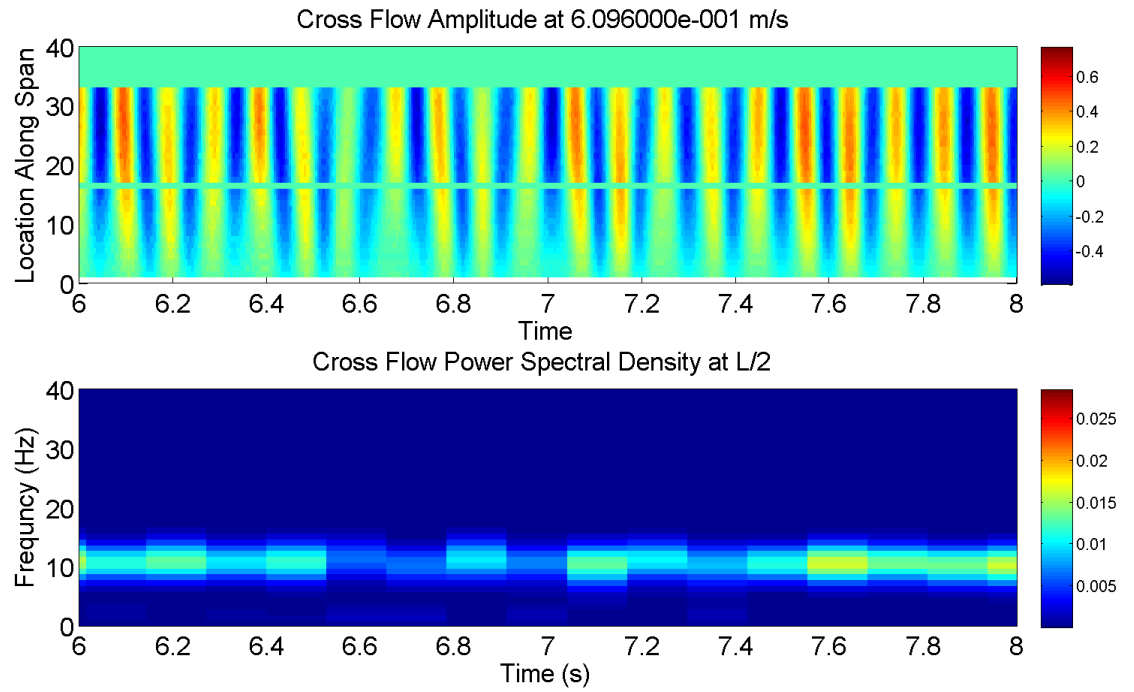

Figure C.14: $\mathrm{Vr}=6.127(\mathrm{U}=0.61 \mathrm{~m} / \mathrm{s})$, Time history of amplitude and PSD of the CF Direction 

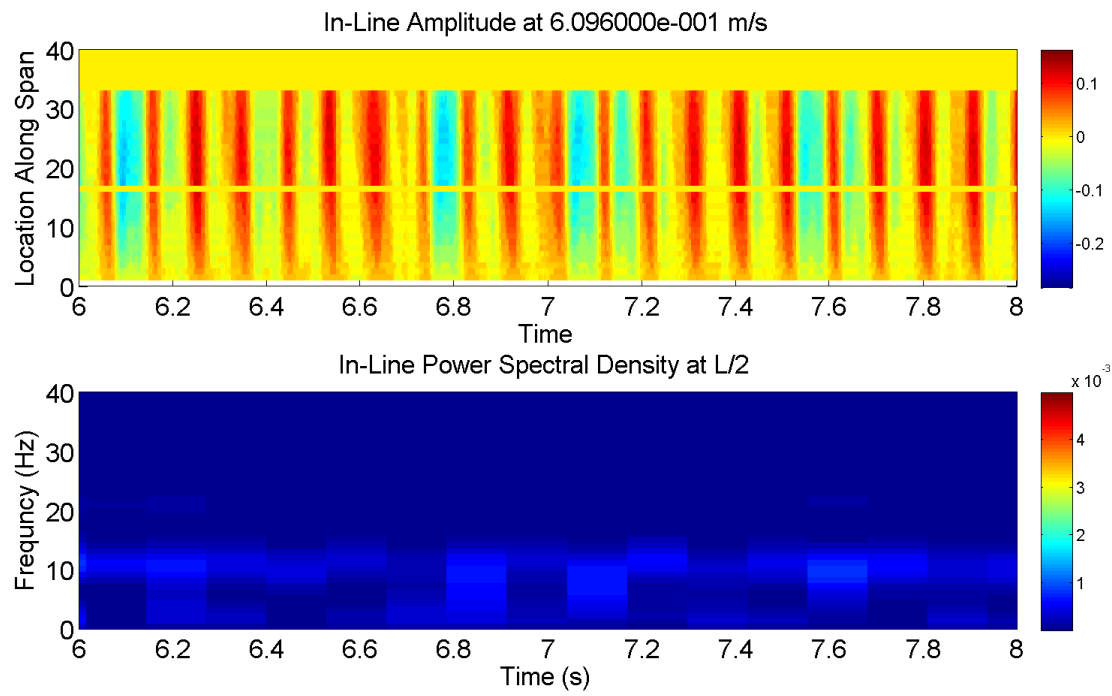

Figure C.15: $\mathrm{Vr}=6.127(\mathrm{U}=0.61 \mathrm{~m} / \mathrm{s})$, Time history of amplitude and PSD of the IL Direction
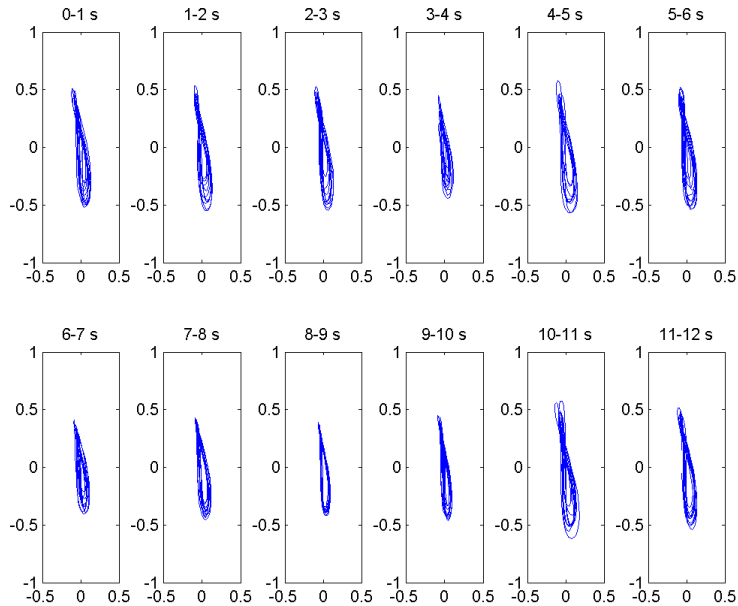

Figure C.16: $\mathrm{Vr}=6.127(\mathrm{U}=0.61 \mathrm{~m} / \mathrm{s})$, orbital patterns $0-12 \mathrm{sec}$ 


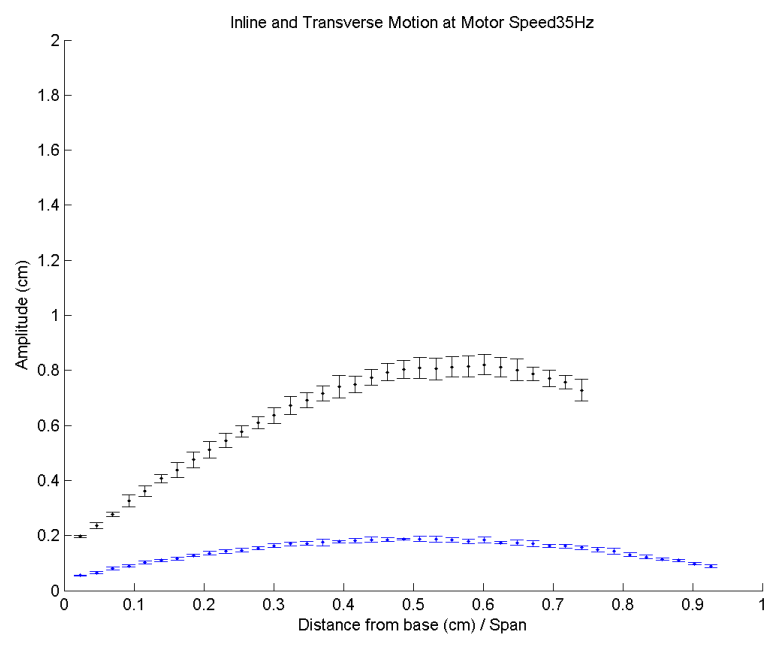

Figure C.17: $\mathrm{Vr}=6.13(\mathrm{U}=0.63 \mathrm{~m} / \mathrm{s})$, Spanwise shape of in-line (Black) and cross-flow (Blue) directions
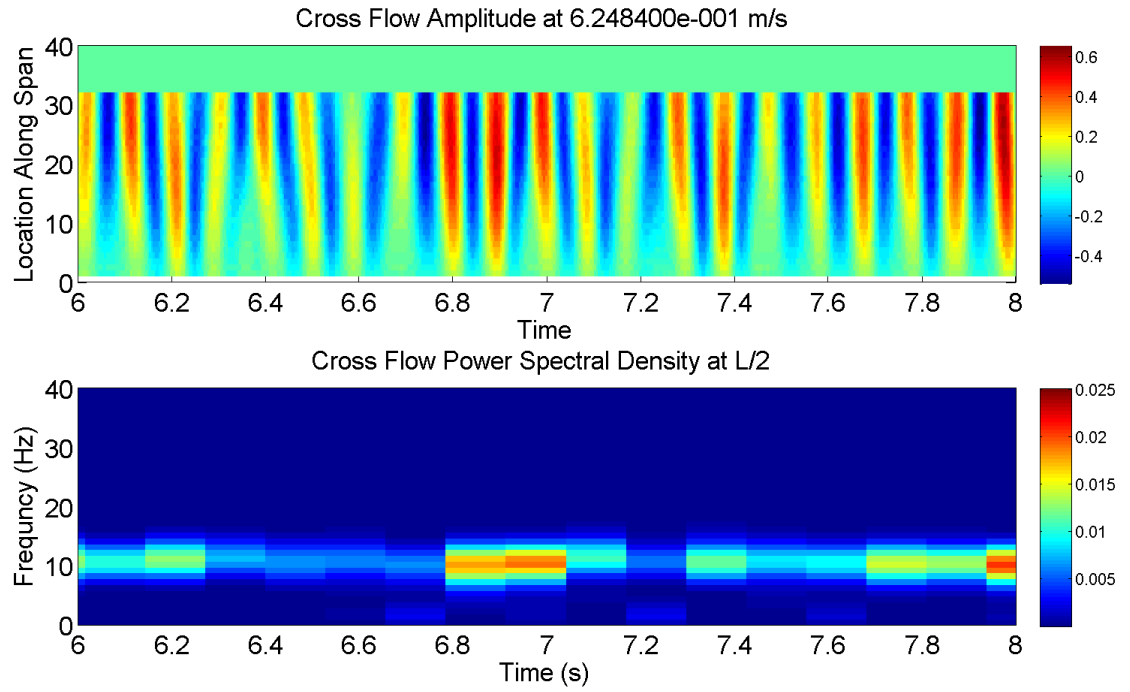

Figure C.18: $\mathrm{Vr}=6.13(\mathrm{U}=0.63 \mathrm{~m} / \mathrm{s})$, Time history of amplitude and PSD of the CF Direction 

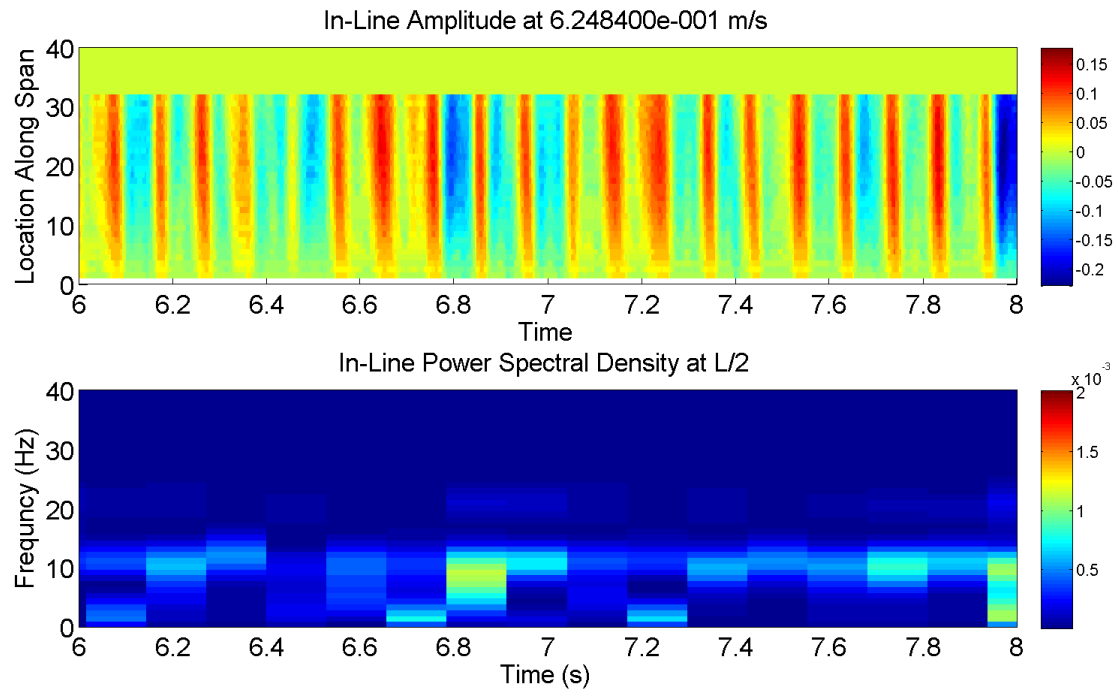

Figure C.19: $\mathrm{Vr}=6.13(\mathrm{U}=0.63 \mathrm{~m} / \mathrm{s})$, Time history of amplitude and PSD of the IL Direction

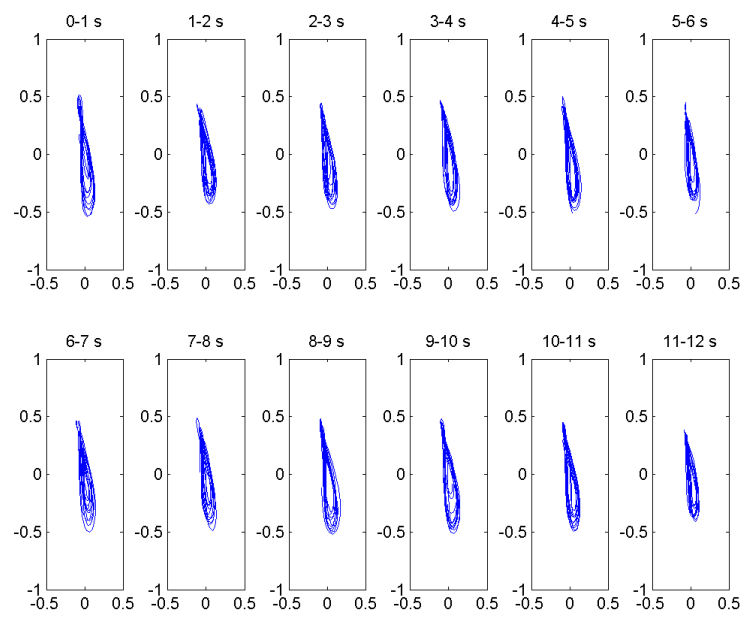

Figure C.20: $\mathrm{Vr}=6.13(\mathrm{U}=0.63 \mathrm{~m} / \mathrm{s})$, orbital patterns $0-12 \mathrm{sec}$ 


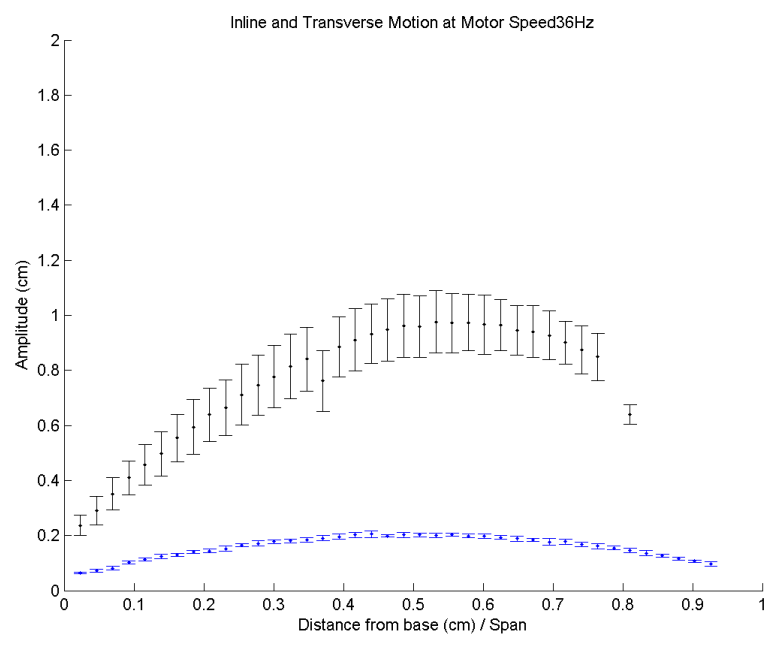

Figure C.21: $\operatorname{Vr}=6.35(\mathrm{U}=0.65 \mathrm{~m} / \mathrm{s})$, Spanwise shape of in-line (Black) and cross-flow (Blue) directions
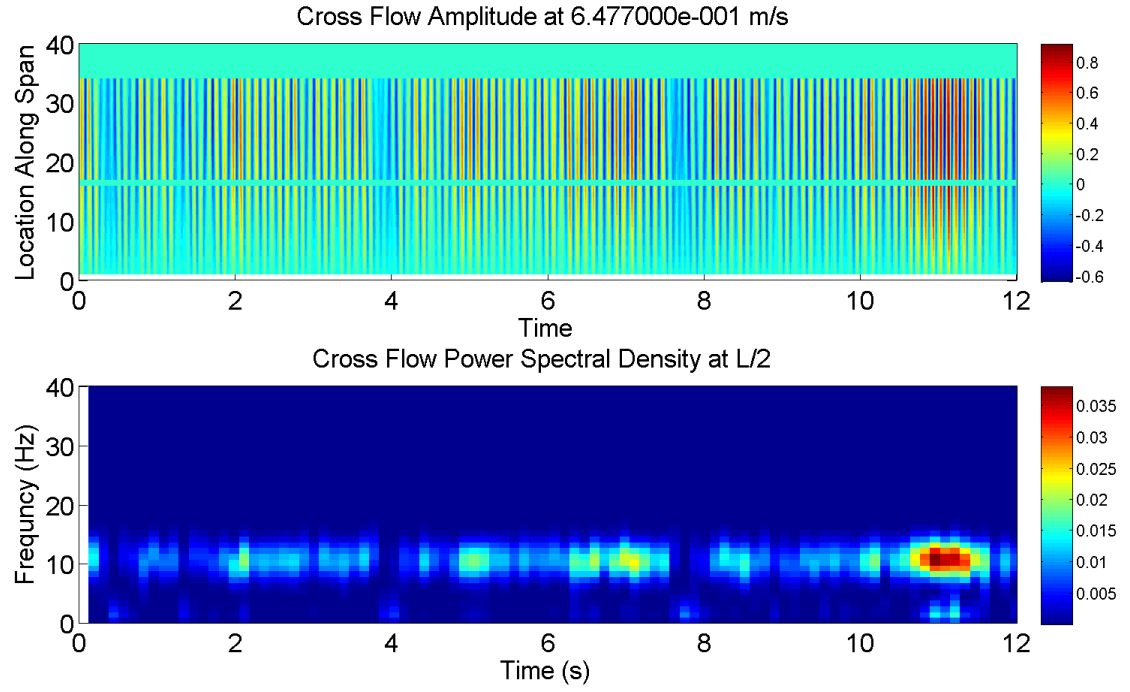

Figure C.22: $\mathrm{Vr}=6.35(\mathrm{U}=0.65 \mathrm{~m} / \mathrm{s})$, Time history of amplitude and PSD of the CF Direction 

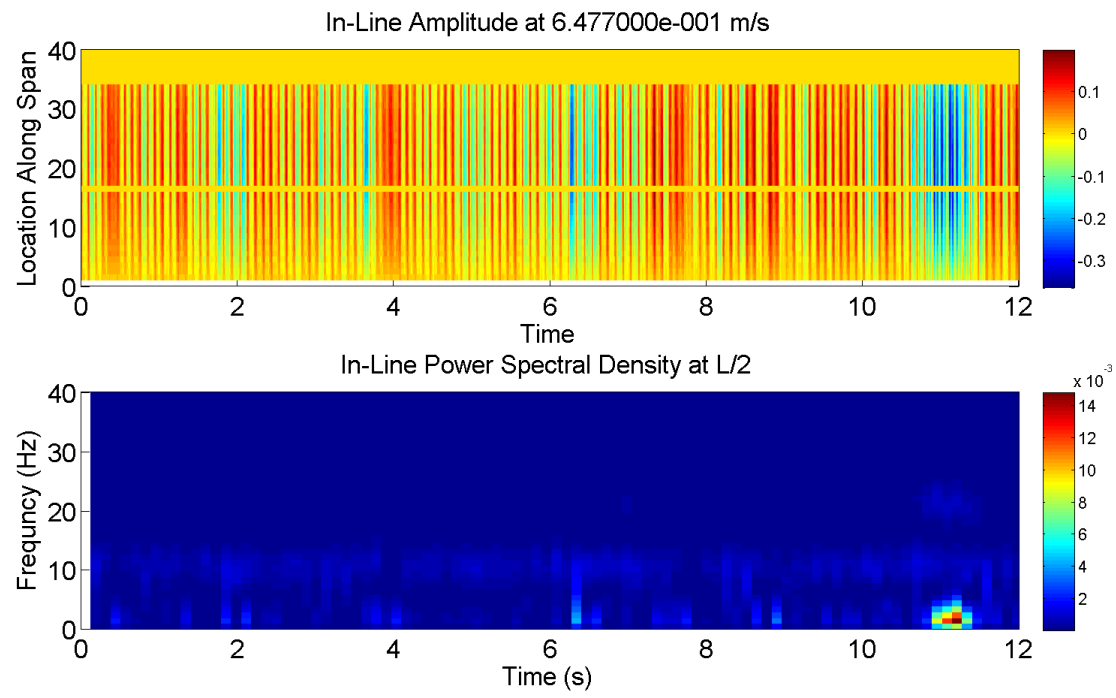

Figure C.23: $\mathrm{Vr}=6.35(\mathrm{U}=0.65 \mathrm{~m} / \mathrm{s})$, Time history of amplitude and PSD of the IL Direction

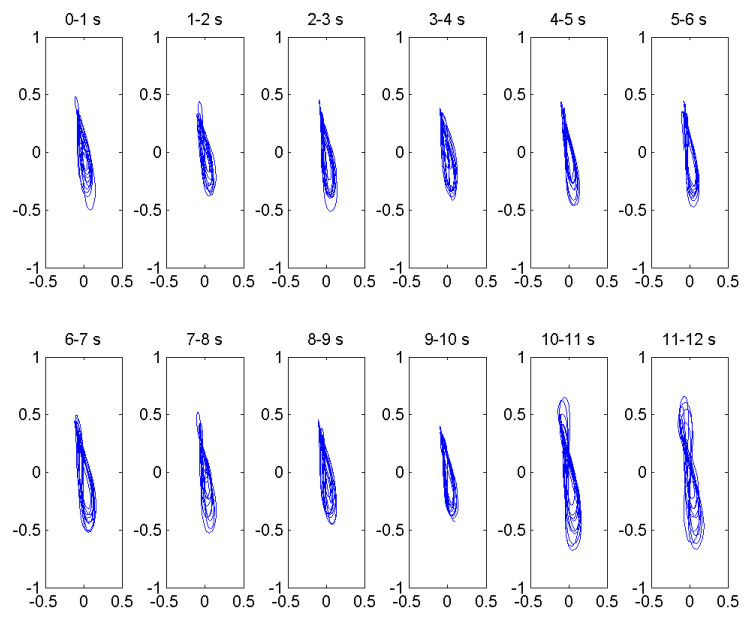

Figure C.24: $\mathrm{Vr}=6.35(\mathrm{U}=0.65 \mathrm{~m} / \mathrm{s})$, orbital patterns $0-12 \mathrm{sec}$ 


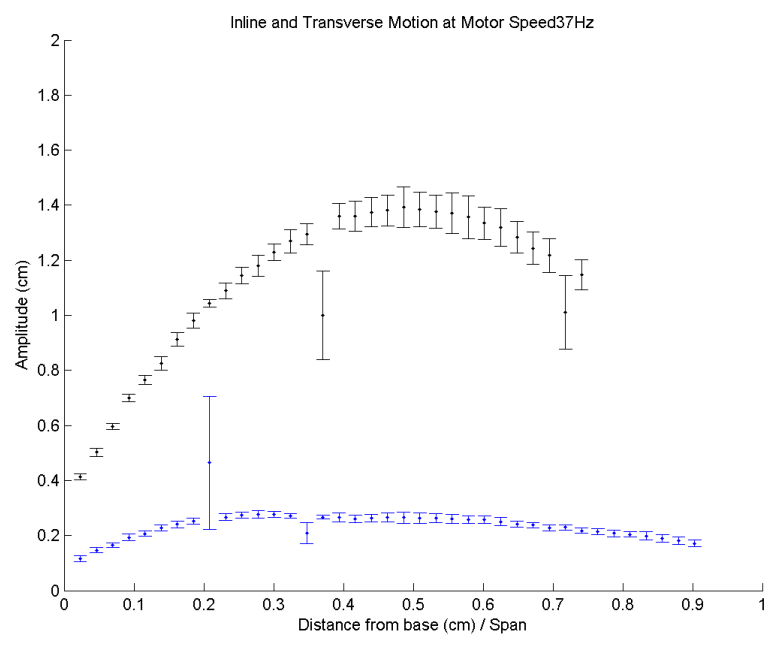

Figure C.25: $\mathrm{Vr}=6.46(\mathrm{U}=0.66 \mathrm{~m} / \mathrm{s})$, Spanwise shape of in-line (Black) and cross-flow (Blue) directions
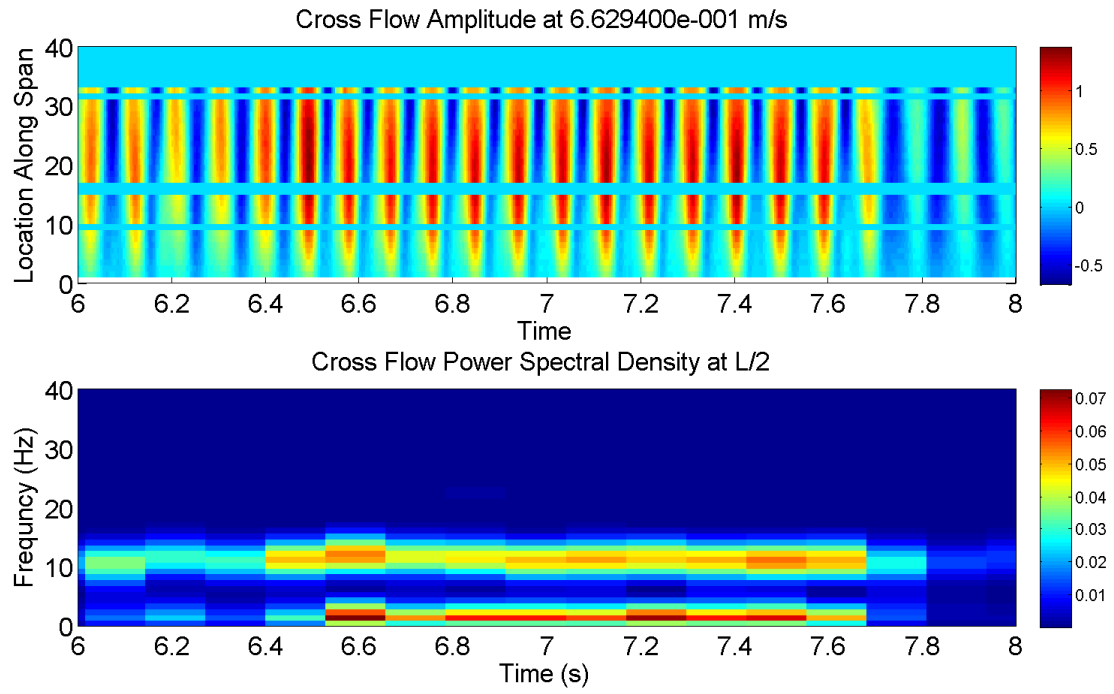

Figure C.26: $\mathrm{Vr}=6.46(\mathrm{U}=0.66 \mathrm{~m} / \mathrm{s})$, Time history of amplitude and PSD of the CF Direction 

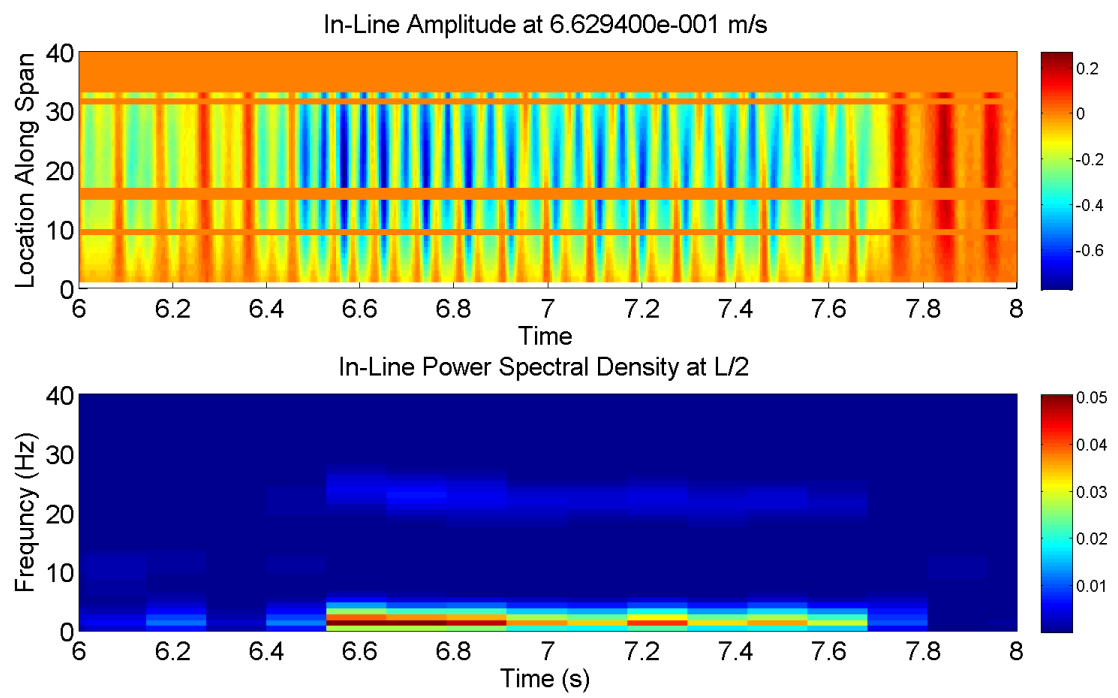

Figure C.27: $\mathrm{Vr}=6.46(\mathrm{U}=0.66 \mathrm{~m} / \mathrm{s})$, Time history of amplitude and PSD of the IL Direction

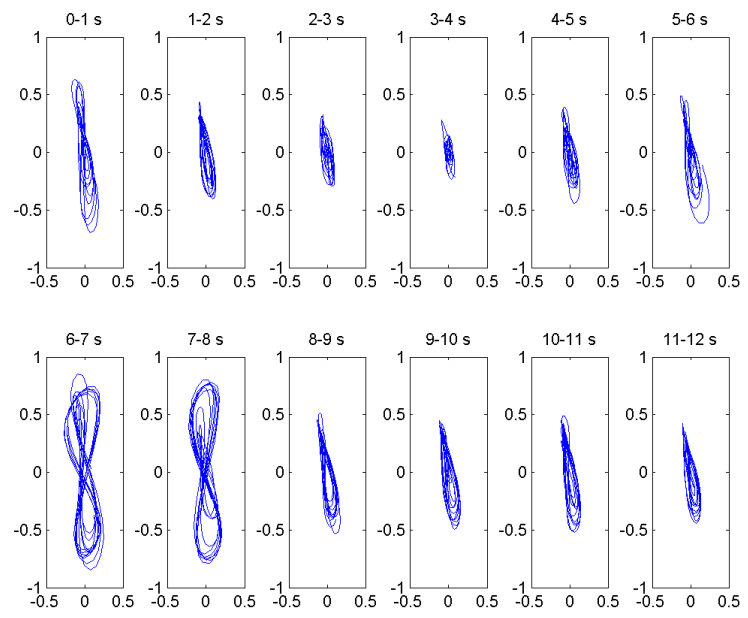

Figure C.28: $\mathrm{Vr}=6.46(\mathrm{U}=0.66 \mathrm{~m} / \mathrm{s})$, orbital patterns $0-12 \mathrm{sec}$ 


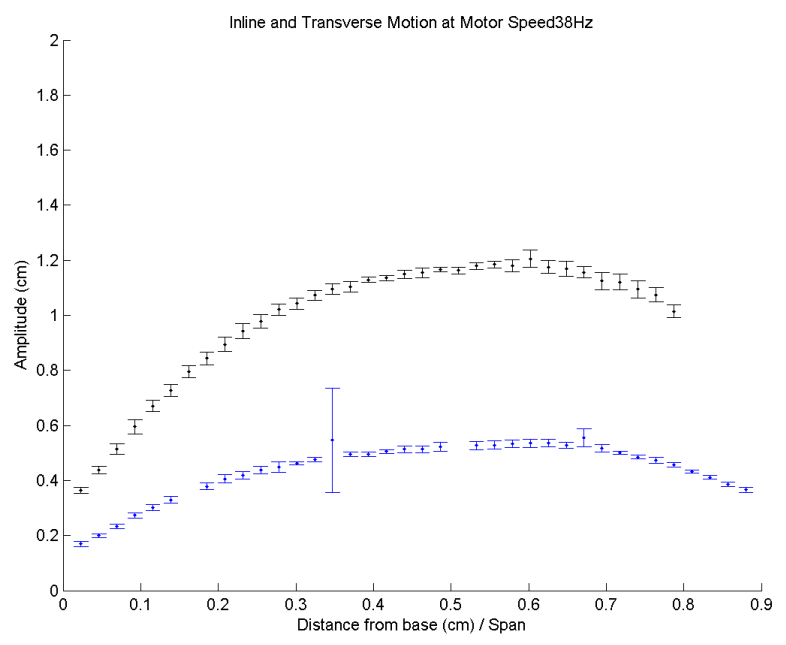

Figure C.29: $\mathrm{Vr}=5.90(\mathrm{U}=0.69 \mathrm{~m} / \mathrm{s})$, Spanwise shape of in-line (Black) and cross-flow (Blue) directions
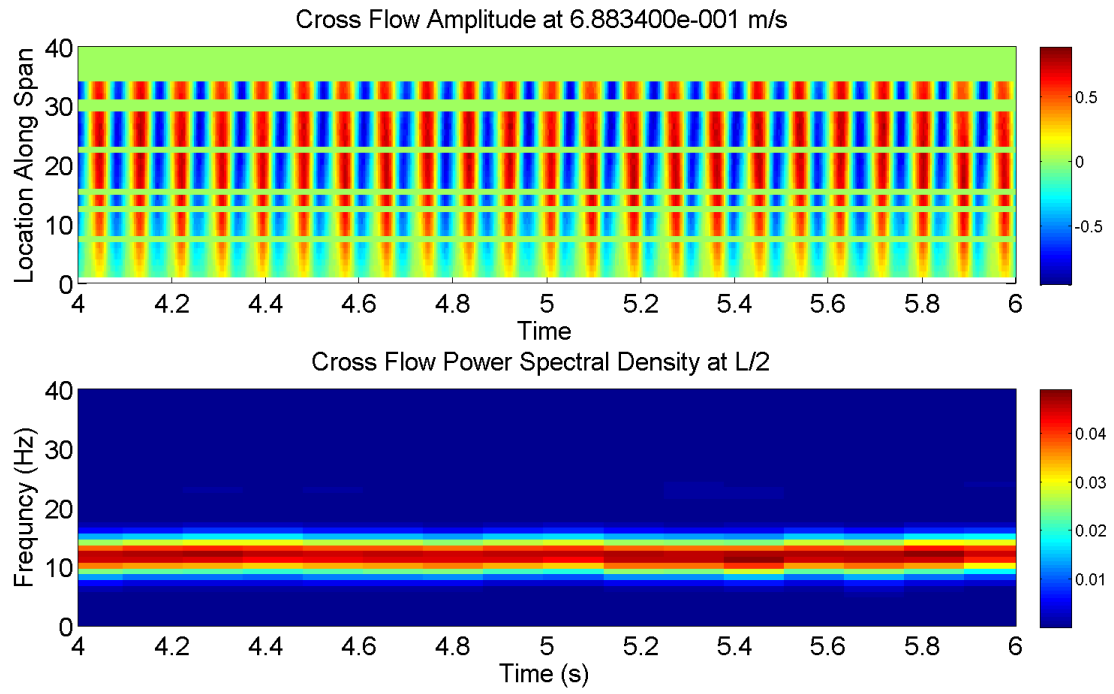

Figure C.30: $\mathrm{Vr}=5.90(\mathrm{U}=0.69 \mathrm{~m} / \mathrm{s})$, Time history of amplitude and PSD of the CF Direction 

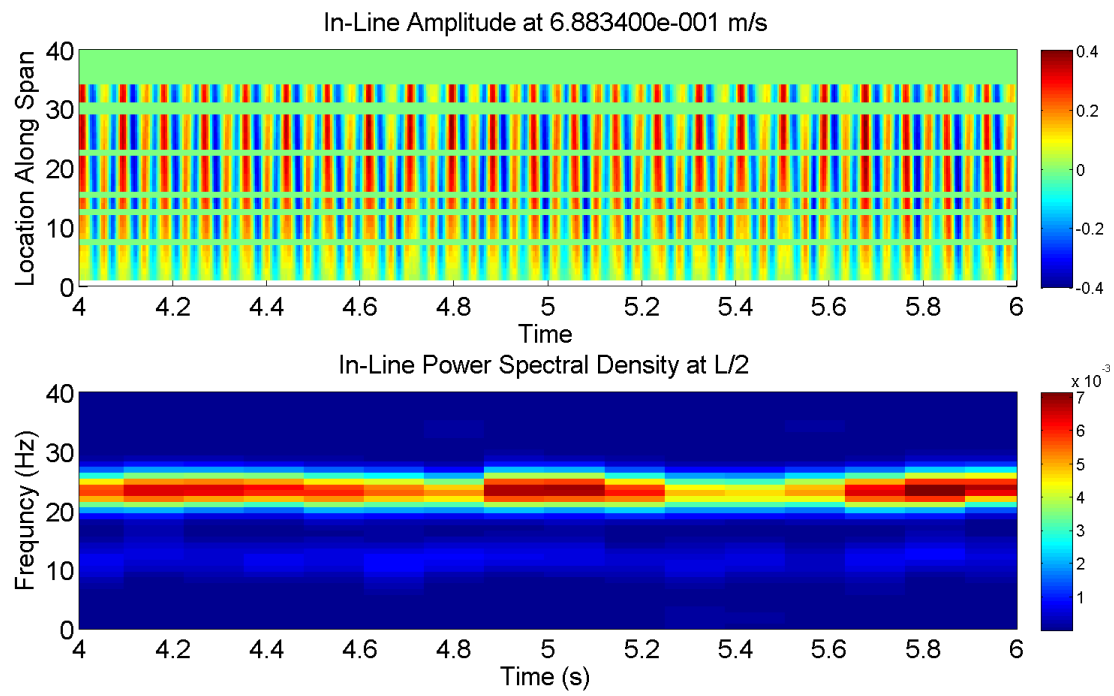

Figure C.31: $\mathrm{Vr}=5.90(\mathrm{U}=0.69 \mathrm{~m} / \mathrm{s})$, Time history of amplitude and PSD of the IL Direction
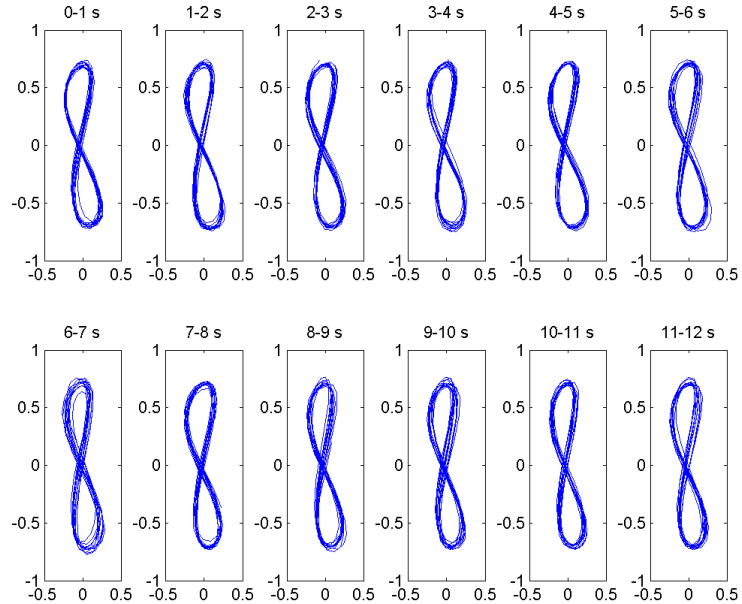

Figure C.32: $\mathrm{Vr}=5.90(\mathrm{U}=0.69 \mathrm{~m} / \mathrm{s})$, orbital patterns $0-12 \mathrm{sec}$ 


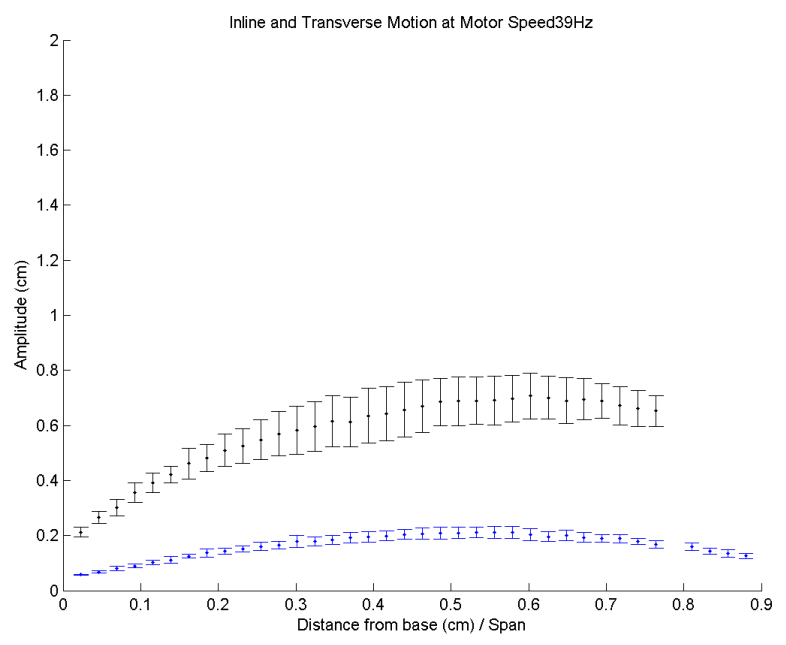

Figure C.33: $\mathrm{Vr}=5.13(\mathrm{U}=0.70 \mathrm{~m} / \mathrm{s})$, Spanwise shape of in-line (Black) and cross-flow (Blue) directions
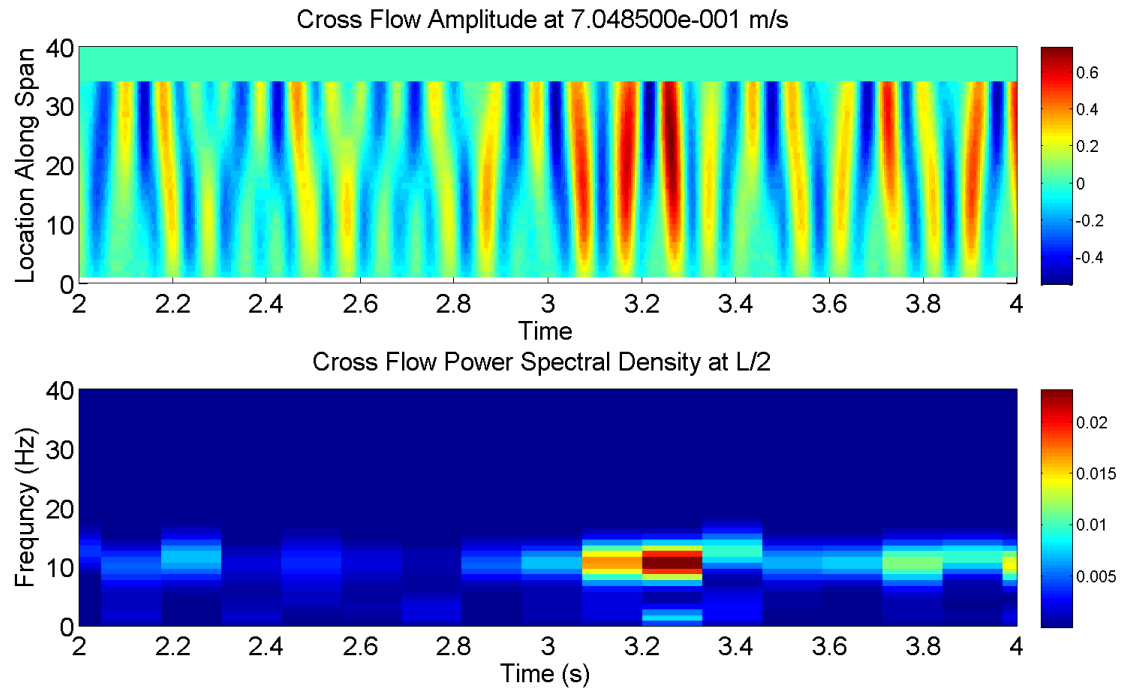

Figure C.34: $\mathrm{Vr}=5.13(\mathrm{U}=0.70 \mathrm{~m} / \mathrm{s})$, Time history of amplitude and PSD of the CF Direction 

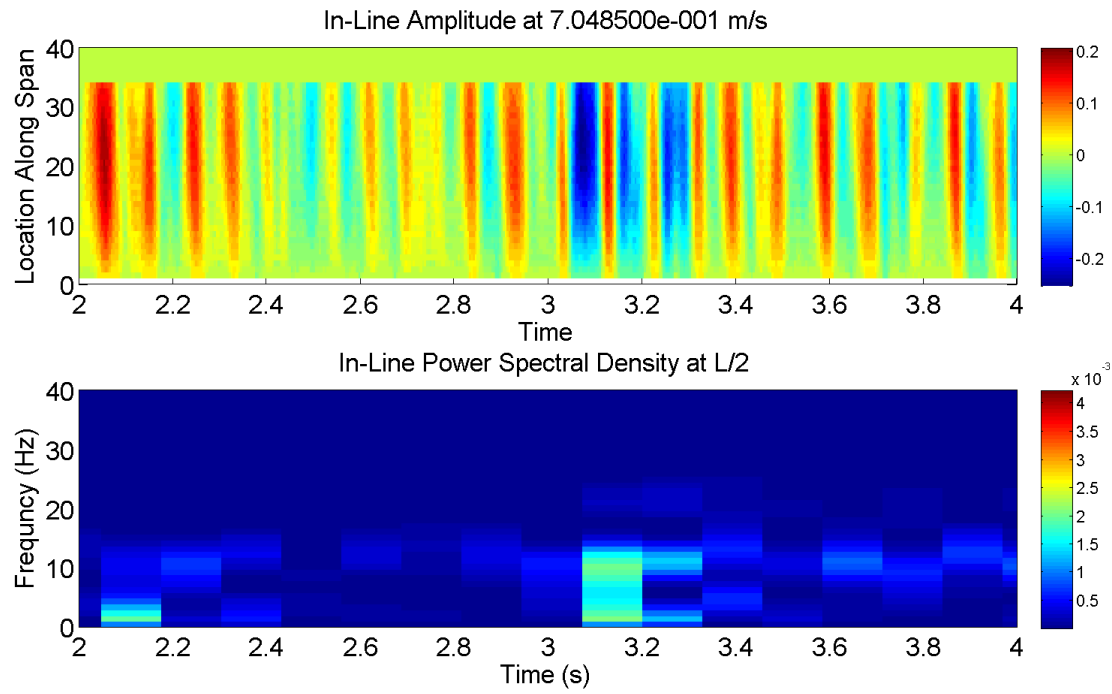

Figure C.35: $\mathrm{Vr}=5.13(\mathrm{U}=0.70 \mathrm{~m} / \mathrm{s})$, Time history of amplitude and PSD of the IL Direction
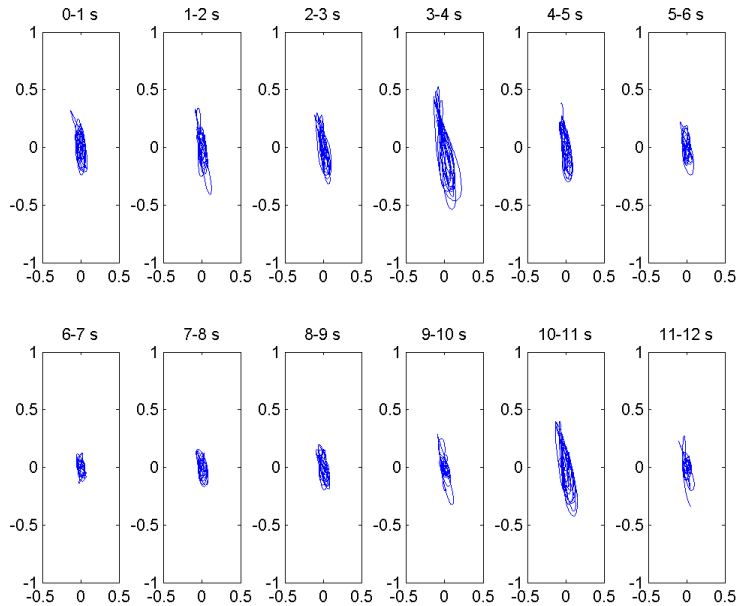

Figure C.36: $\mathrm{Vr}=5.13(\mathrm{U}=0.70 \mathrm{~m} / \mathrm{s})$, orbital patterns $0-12 \mathrm{sec}$ 


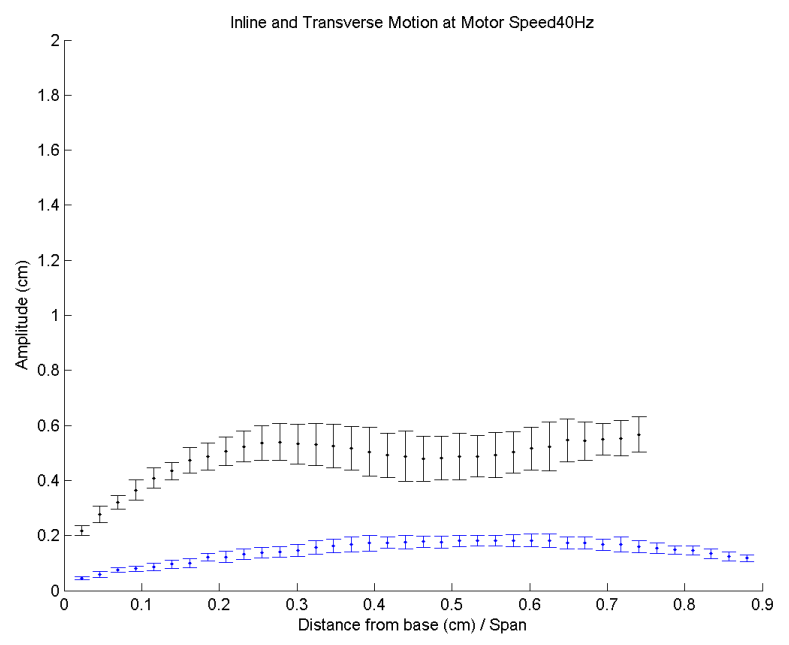

Figure C.37: $3 \mathrm{Vr}=5.10(\mathrm{U}=0.72 \mathrm{~m} / \mathrm{s})$, Spanwise shape of in-line (Black) and cross-flow (Blue) directions
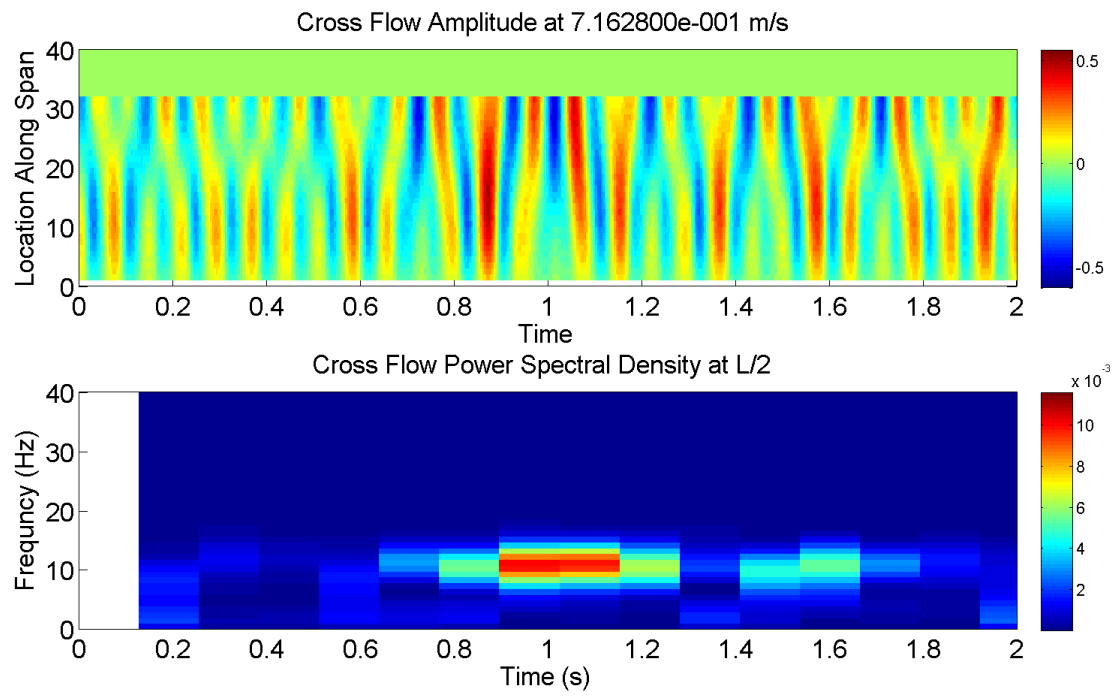

Figure C.38: $\mathrm{Vr}=5.10(\mathrm{U}=0.72 \mathrm{~m} / \mathrm{s})$, Time history of amplitude and PSD of the CF Direction 

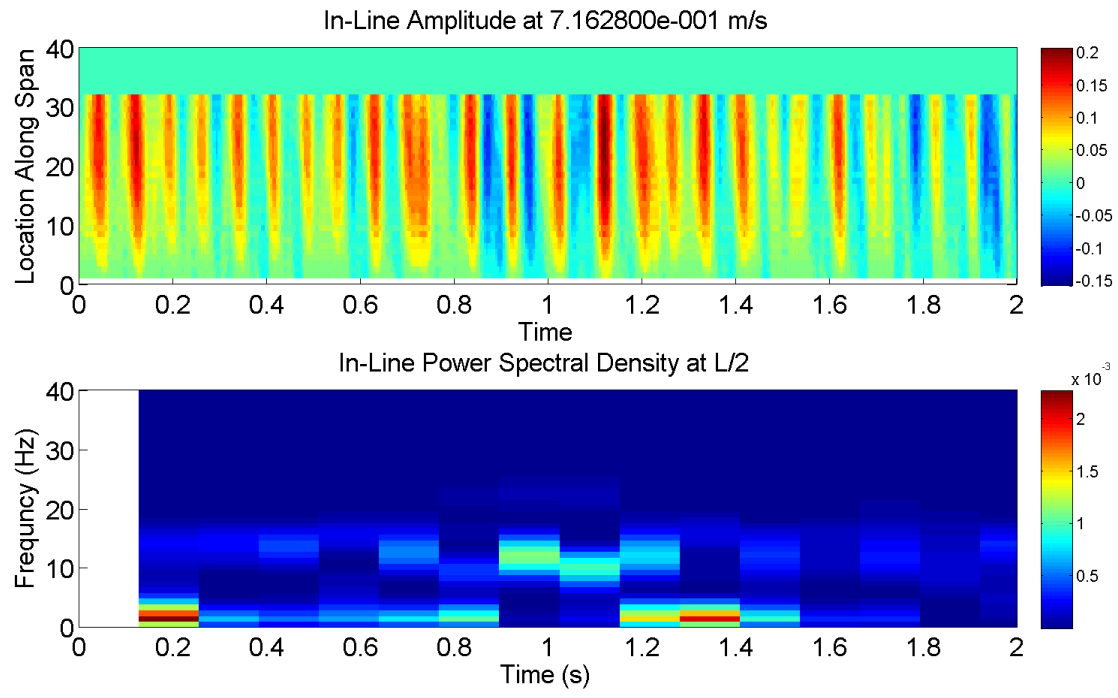

Figure C.39: $\mathrm{Vr}=5.10(\mathrm{U}=0.72 \mathrm{~m} / \mathrm{s})$, Time history of amplitude and PSD of the IL Direction
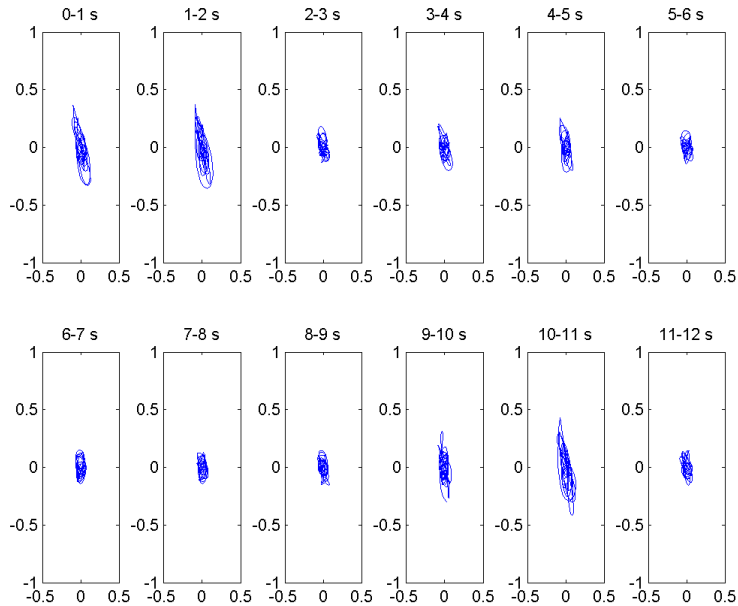

Figure C.40: $\mathrm{Vr}=5.10(\mathrm{U}=0.72 \mathrm{~m} / \mathrm{s})$, orbital patterns $0-12 \mathrm{sec}$ 


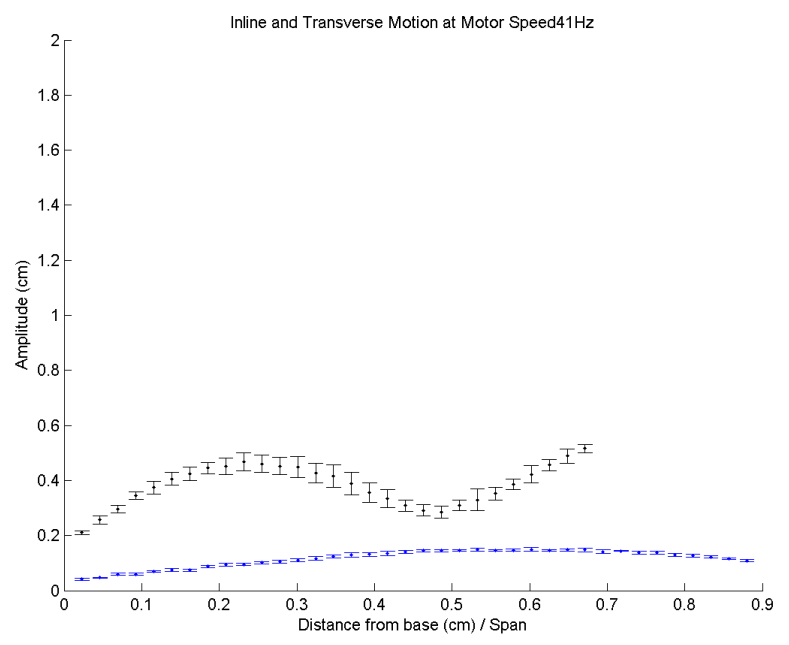

Figure C.41: $\mathrm{Vr}=5.08(\mathrm{U}=0.73 \mathrm{~m} / \mathrm{s})$, Spanwise shape of in-line (Black) and cross-flow (Blue) directions
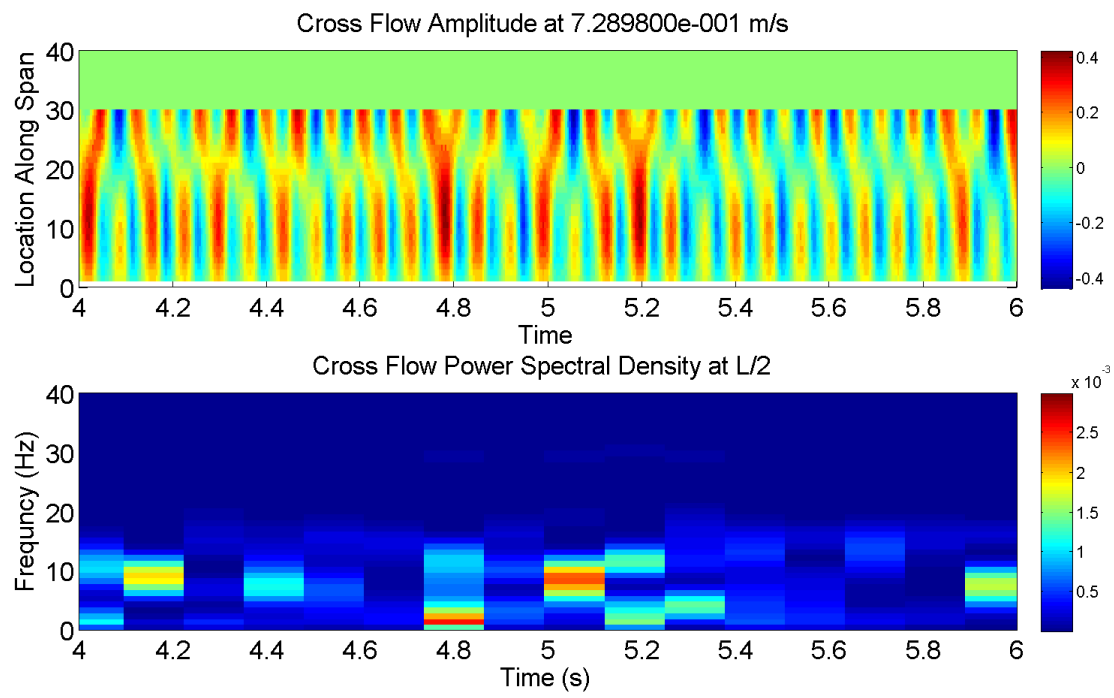

Figure C.42: $\mathrm{Vr}=5.08(\mathrm{U}=0.73 \mathrm{~m} / \mathrm{s})$, Time history of amplitude and PSD of the CF Direction 

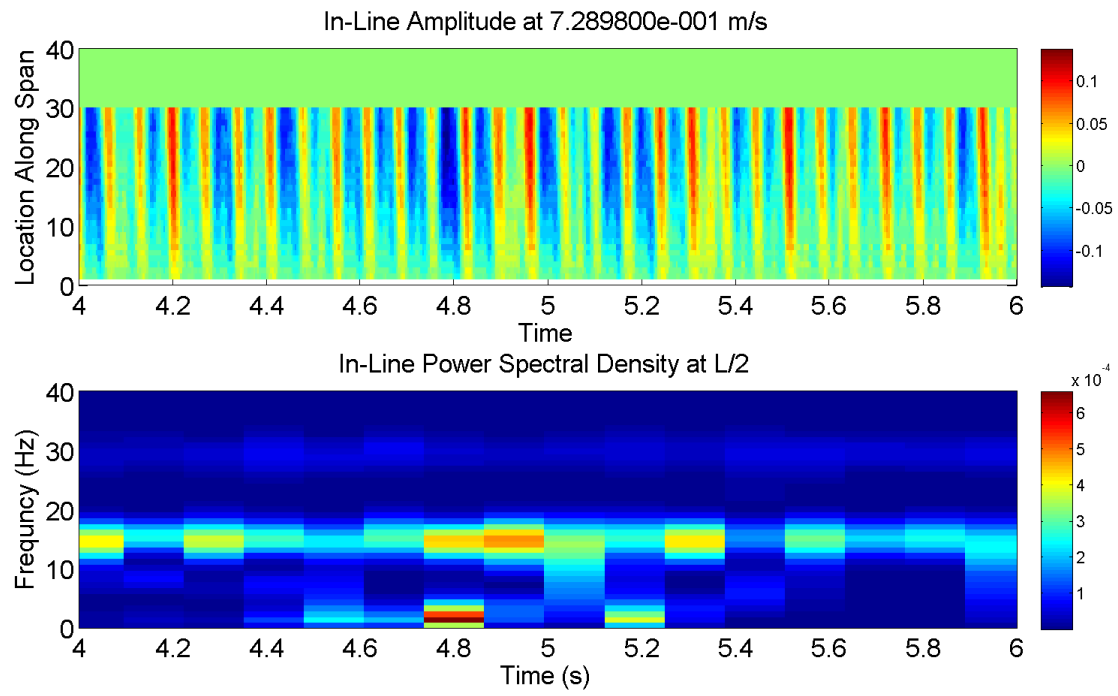

Figure C.43: $\mathrm{Vr}=5.08(\mathrm{U}=0.73 \mathrm{~m} / \mathrm{s})$, Time history of amplitude and PSD of the IL Direction
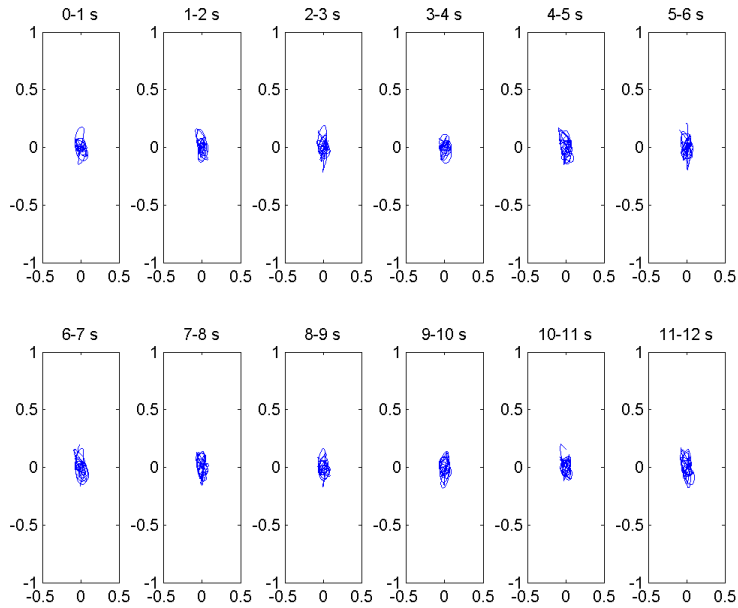

Figure C.44: $\mathrm{Vr}=5.08(\mathrm{U}=0.73 \mathrm{~m} / \mathrm{s})$, orbital patterns $0-12 \mathrm{sec}$ 


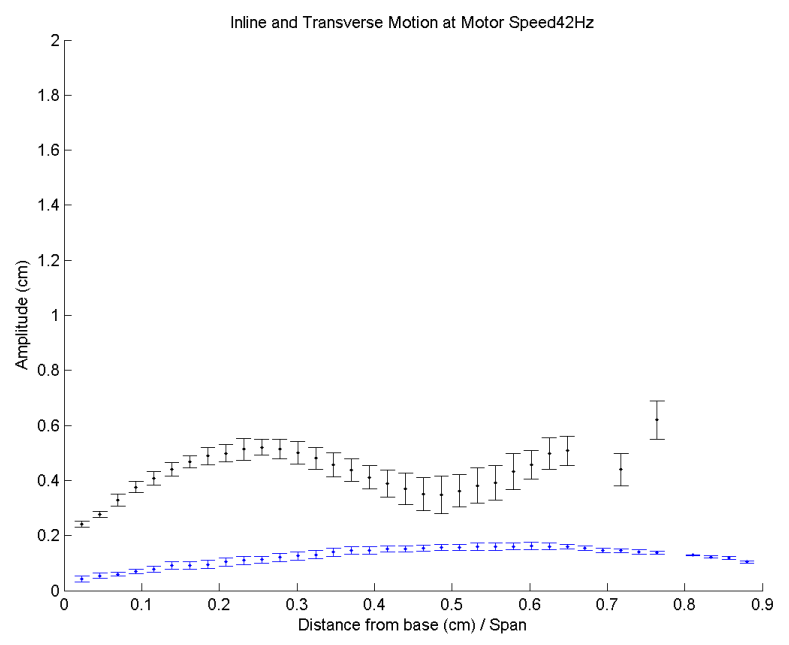

Figure C.45: $\mathrm{Vr}=5.09(\mathrm{U}=0.75 \mathrm{~m} / \mathrm{s})$, Spanwise shape of in-line (Black) and cross-flow (Blue) directions
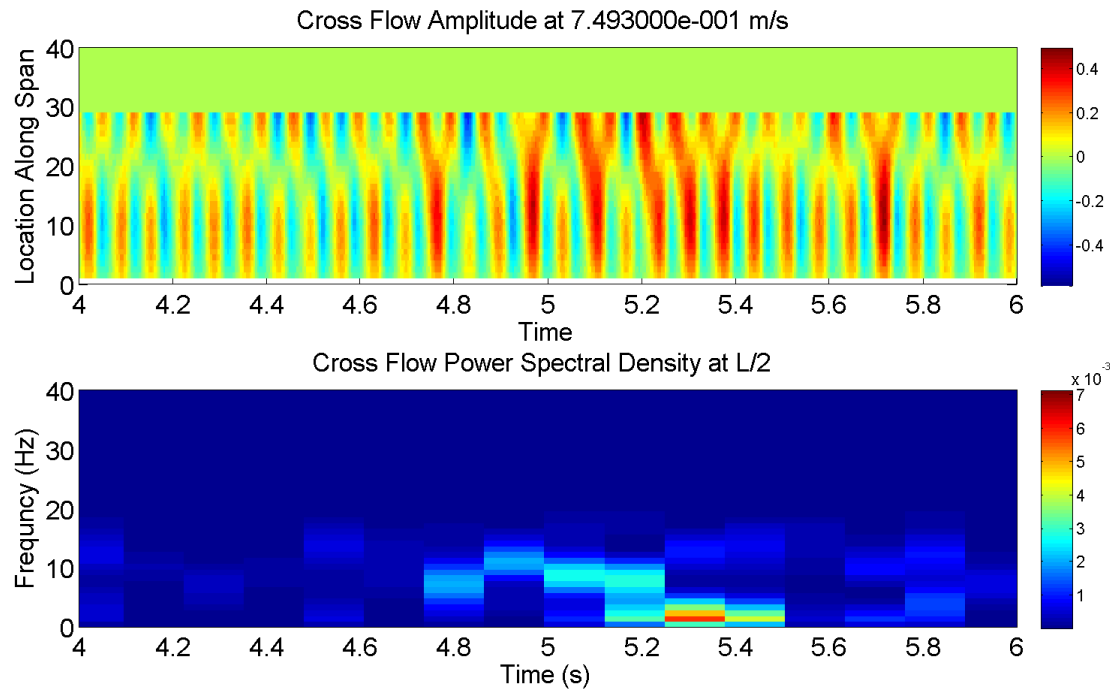

Figure C.46: $\mathrm{Vr}=5.09(\mathrm{U}=0.75 \mathrm{~m} / \mathrm{s})$, Time history of amplitude and PSD of the CF Direction 

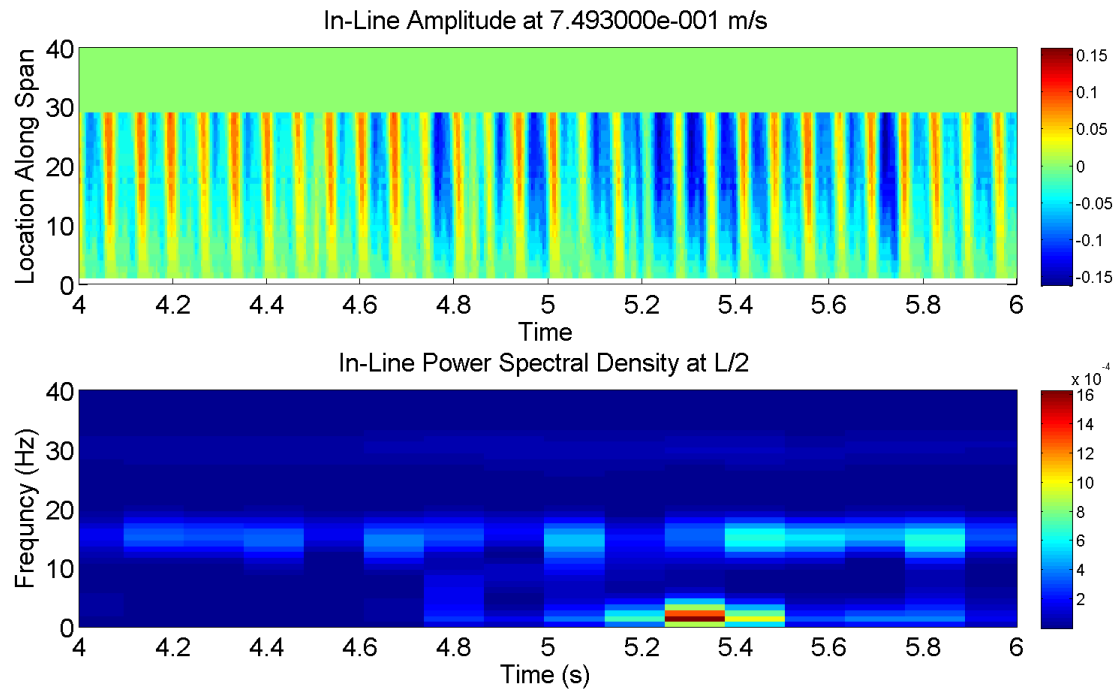

Figure C.47: $\mathrm{Vr}=5.09(\mathrm{U}=0.75 \mathrm{~m} / \mathrm{s})$, Time history of amplitude and PSD of the IL Direction
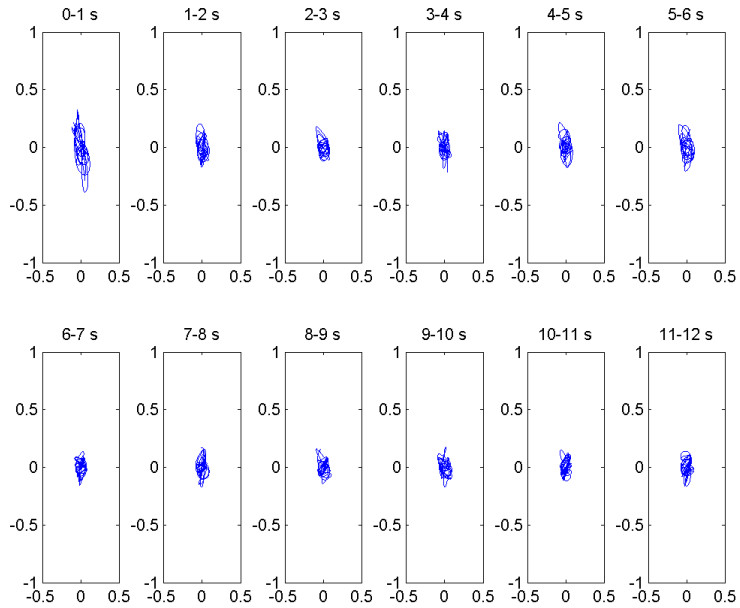

Figure C.48: $\mathrm{Vr}=5.09(\mathrm{U}=0.75 \mathrm{~m} / \mathrm{s})$, orbital patterns $0-12 \mathrm{sec}$ 


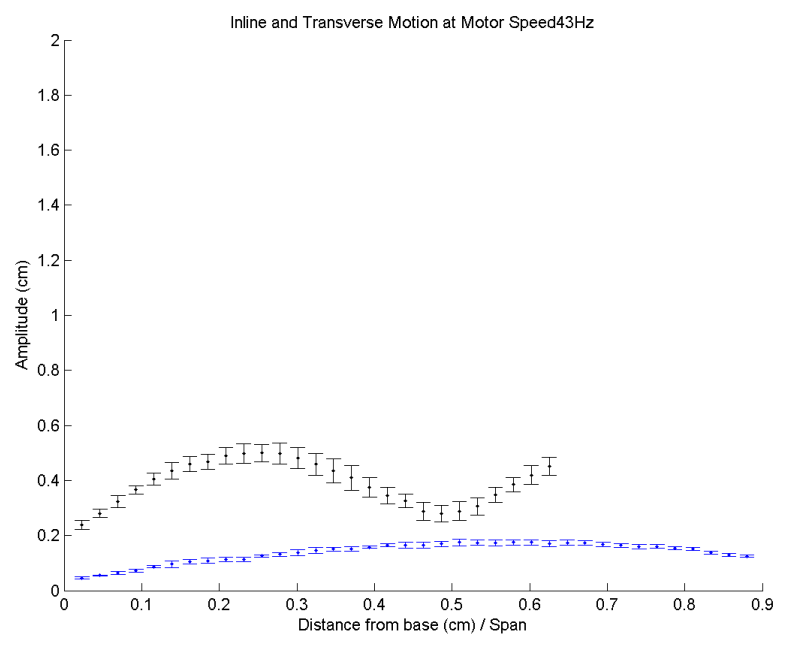

Figure C.49: $\operatorname{Vr}=5.14(\mathrm{U}=0.77 \mathrm{~m} / \mathrm{s})$, Spanwise shape of in-line (Black) and cross-flow (Blue) directions
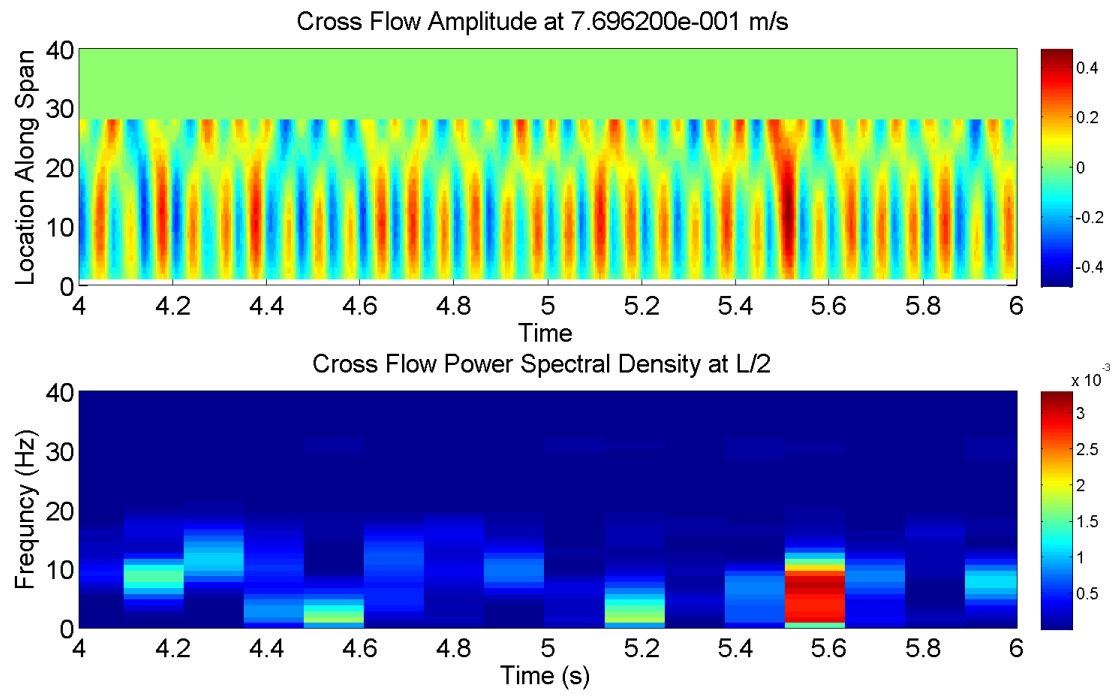

Figure C.50: $\mathrm{Vr}=5.14(\mathrm{U}=0.77 \mathrm{~m} / \mathrm{s})$, Time history of amplitude and PSD of the CF Direction 

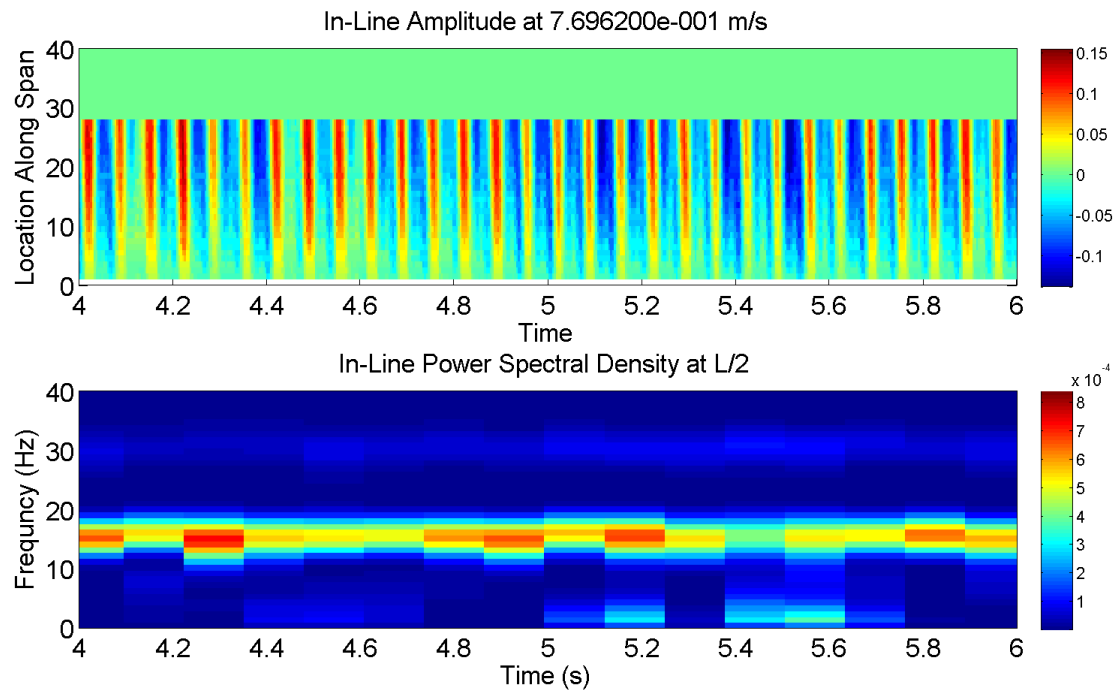

Figure C.51: $\mathrm{Vr}=5.14(\mathrm{U}=0.77 \mathrm{~m} / \mathrm{s})$, Time history of amplitude and PSD of the IL Direction
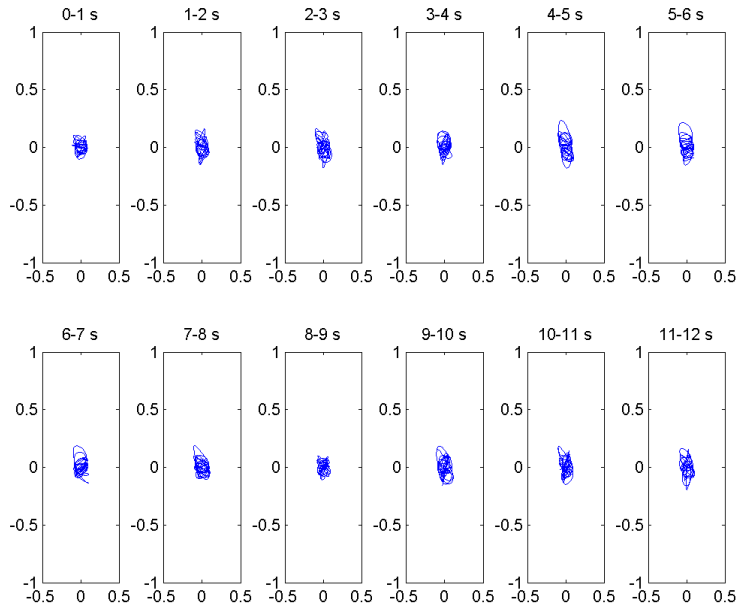

Figure C.52: $\mathrm{Vr}=5.14(\mathrm{U}=0.77 \mathrm{~m} / \mathrm{s})$, orbital patterns $0-12 \mathrm{sec}$ 


\section{APPENDIX D}

\section{Appendix D}

Third Mode In-line and First Mode Cross-Flow (3-1 Cylinder Results)

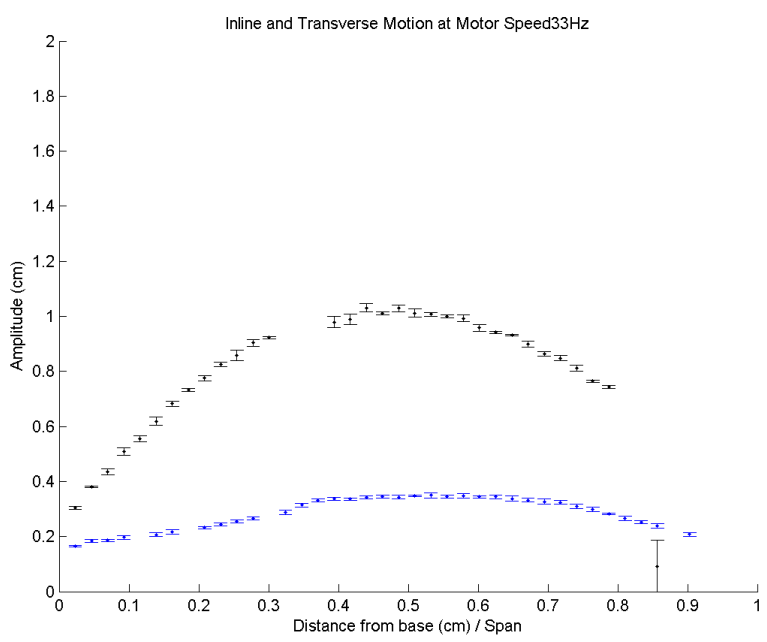

Figure D.1: $\mathrm{Vr}=5.11(\mathrm{U}=0.59 \mathrm{~m} / \mathrm{s})$ Spanwise shape of in-line (Black) and crossflow (Blue) directions
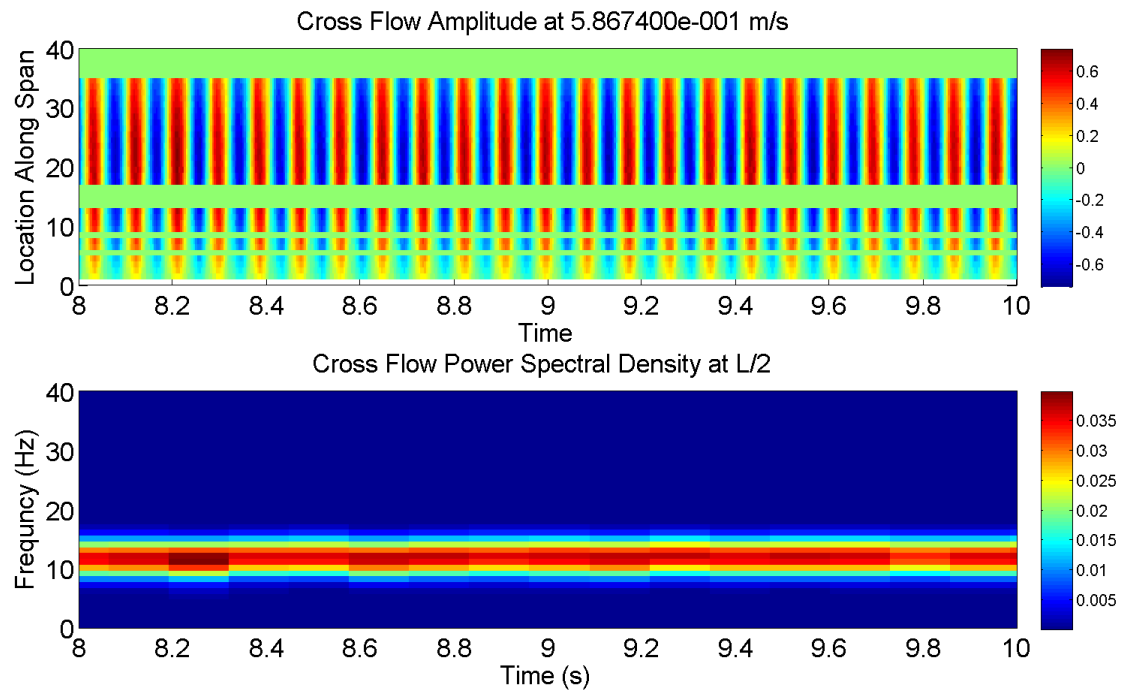

Figure D.2: $\mathrm{Vr}=5.11(\mathrm{U}=0.59 \mathrm{~m} / \mathrm{s})$ Time history of amplitude and PSD of the CF Direction 

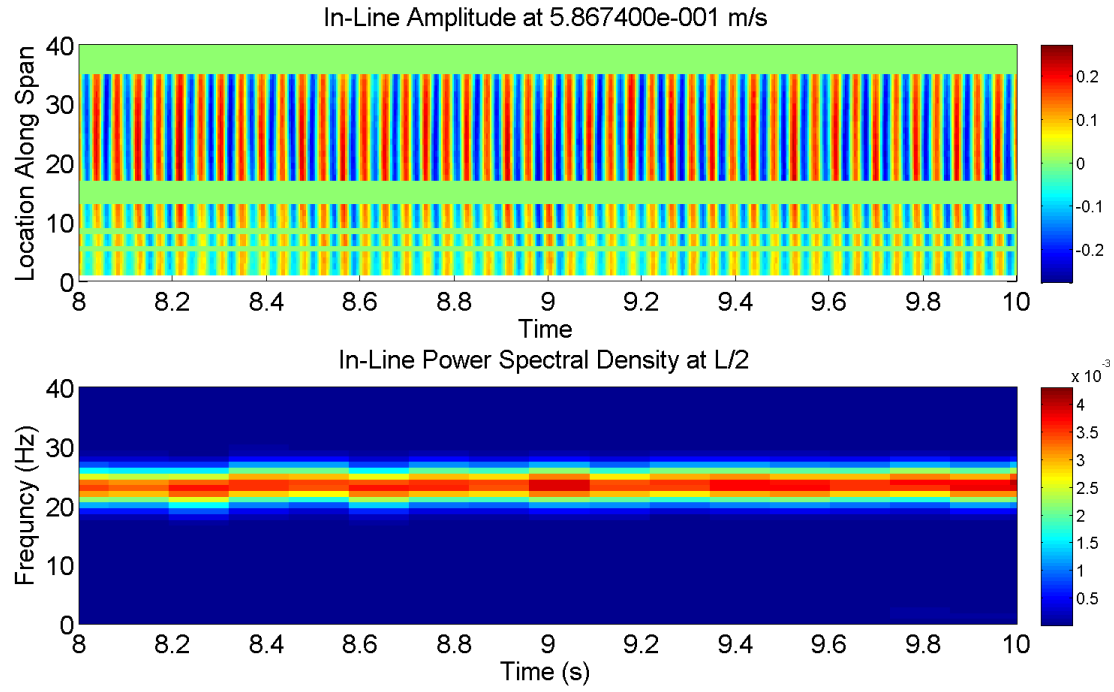

Figure D.3: $\mathrm{Vr}=5.11(\mathrm{U}=0.59 \mathrm{~m} / \mathrm{s})$ Time history of amplitude and PSD of the IL Direction

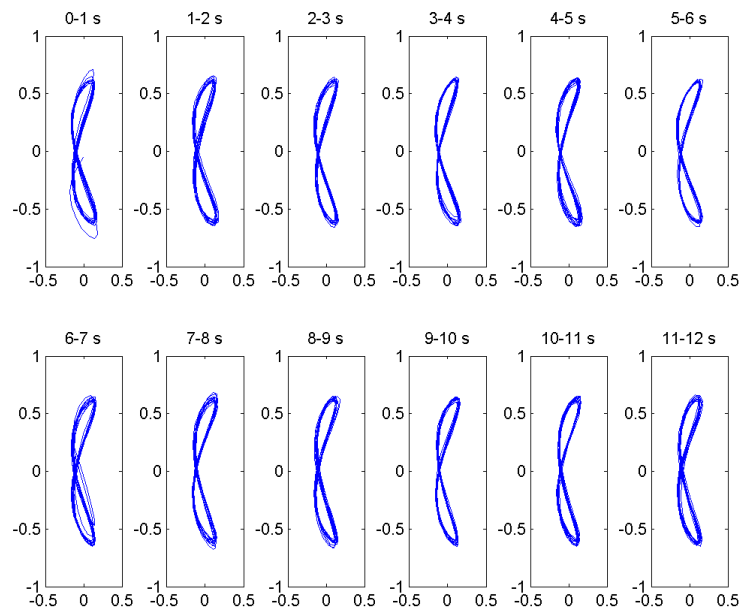

Figure D.4: $\mathrm{Vr}=5.11(\mathrm{U}=0.59 \mathrm{~m} / \mathrm{s})$ orbital patterns $0-12 \mathrm{sec}$ 


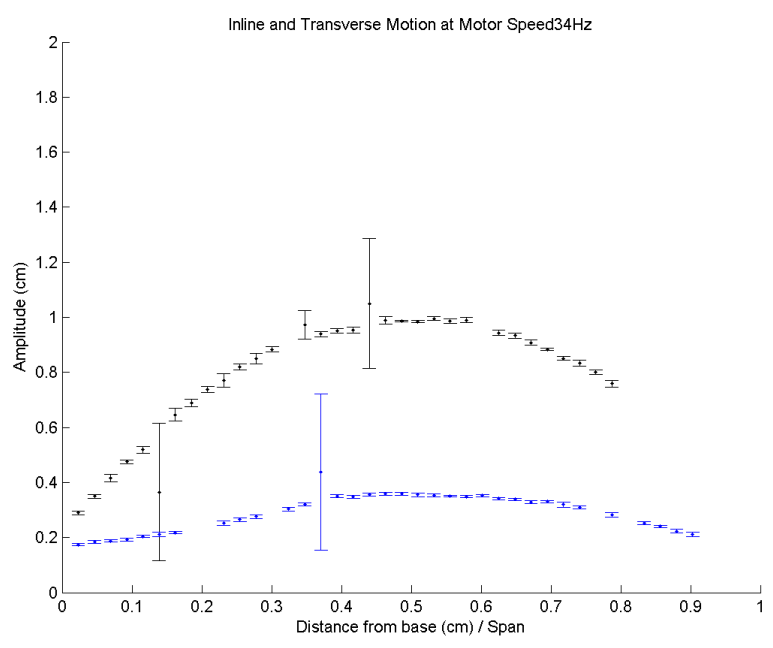

Figure D.5: $\mathrm{Vr}=5.25(\mathrm{U}=0.60 \mathrm{~m} / \mathrm{s})$ Spanwise shape of in-line (Black) and crossflow (Blue) directions
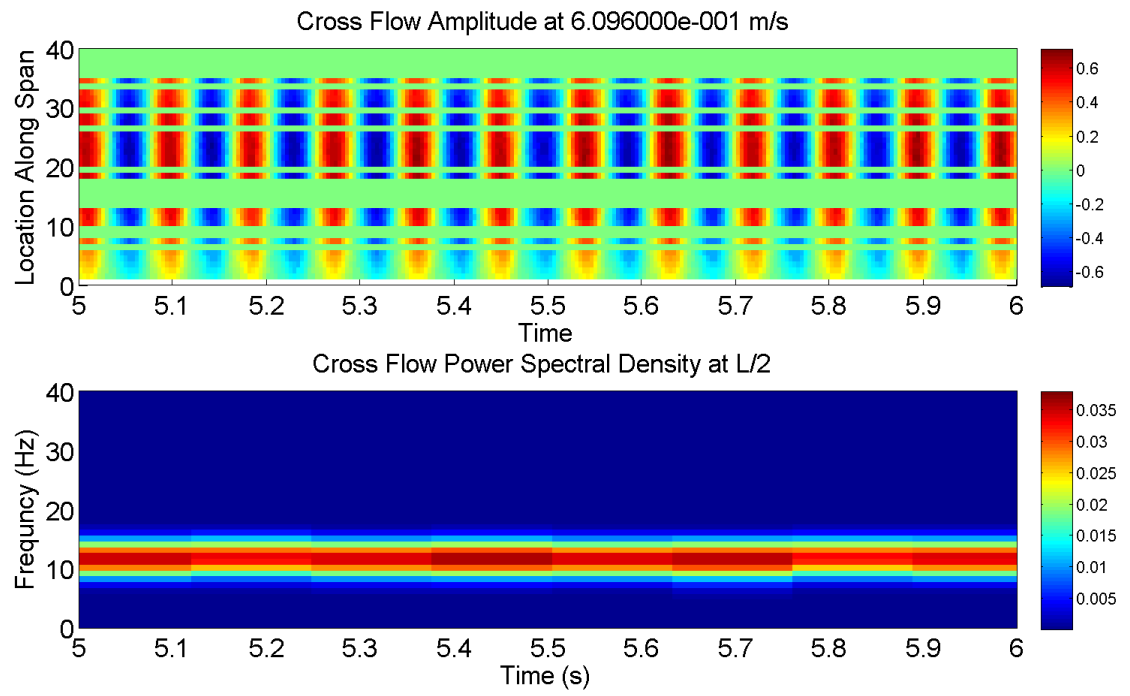

Figure D.6: $\mathrm{Vr}=5.25(\mathrm{U}=0.60 \mathrm{~m} / \mathrm{s})$ Time history of amplitude and PSD of the CF Direction 

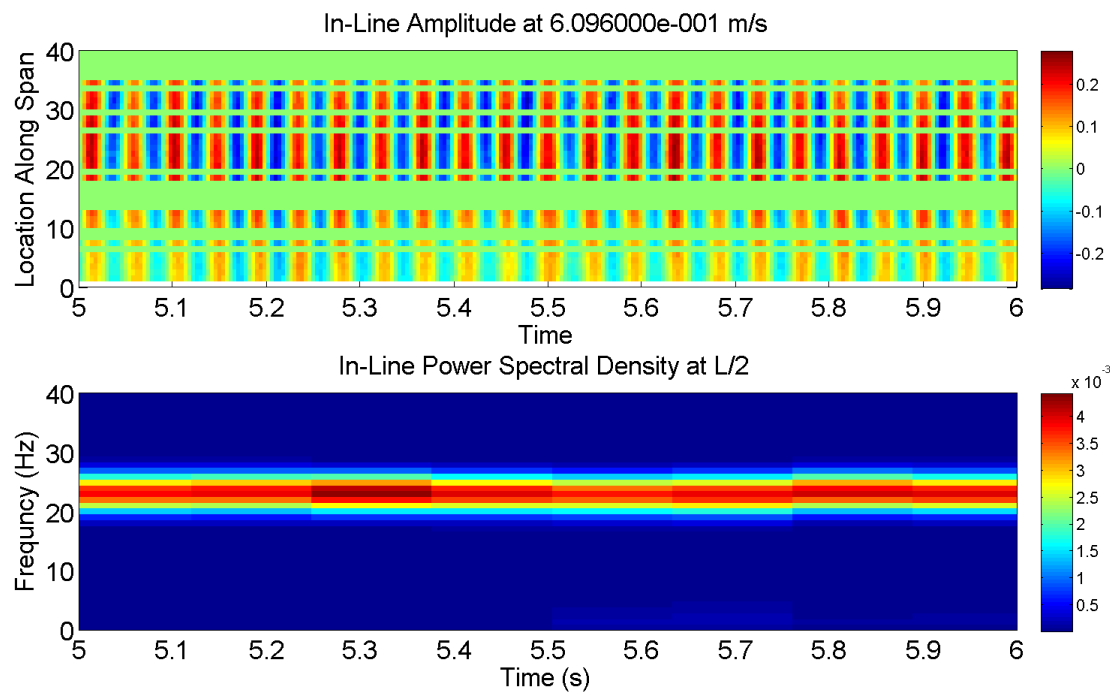

Figure D.7: $\mathrm{Vr}=5.25(\mathrm{U}=0.60 \mathrm{~m} / \mathrm{s})$ Time history of amplitude and PSD of the IL Direction
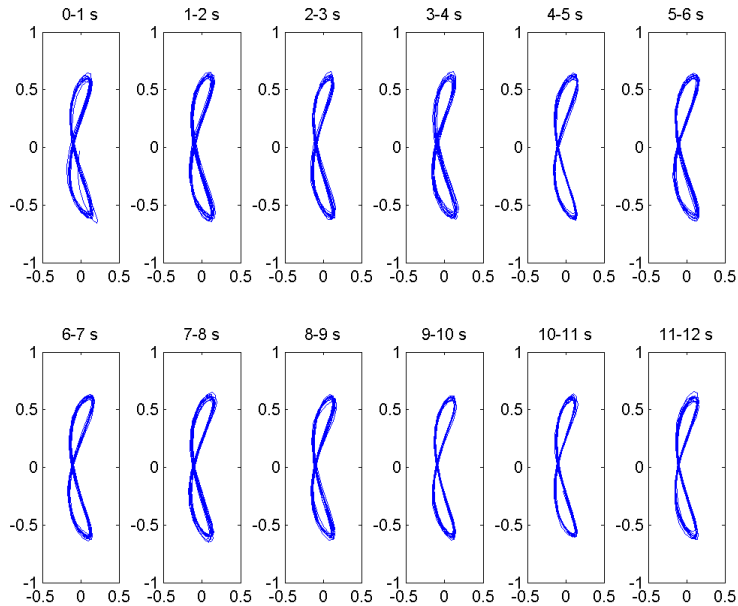

Figure D.8: $\mathrm{Vr}=5.25(\mathrm{U}=0.60 \mathrm{~m} / \mathrm{s})$ orbital patterns $0-12 \mathrm{sec}$ 


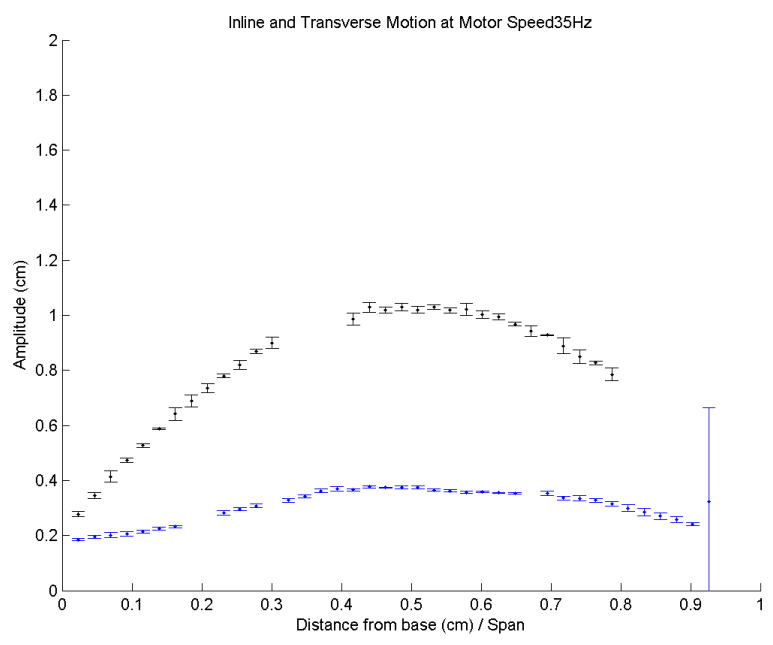

Figure D.9: $\mathrm{Vr}=5.35(\mathrm{U}=0.62 \mathrm{~m} / \mathrm{s})$ Spanwise shape of in-line (Black) and crossflow (Blue) directions
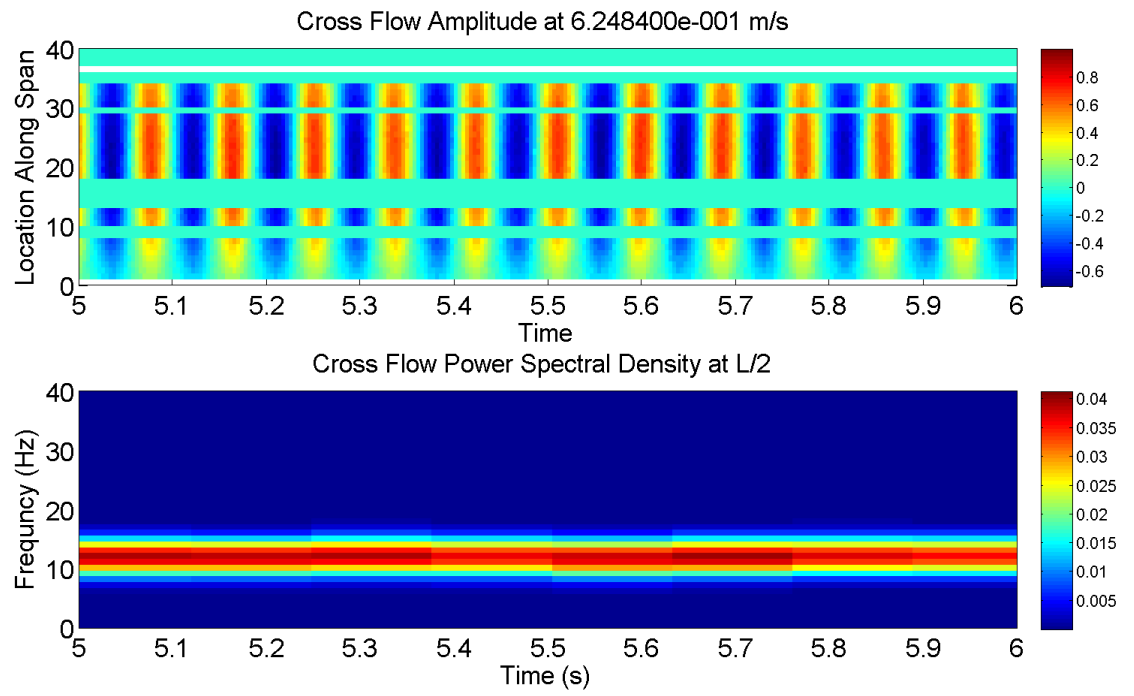

Figure D.10: $\mathrm{Vr}=5.35(\mathrm{U}=0.62 \mathrm{~m} / \mathrm{s})$ Time history of amplitude and PSD of the CF Direction 

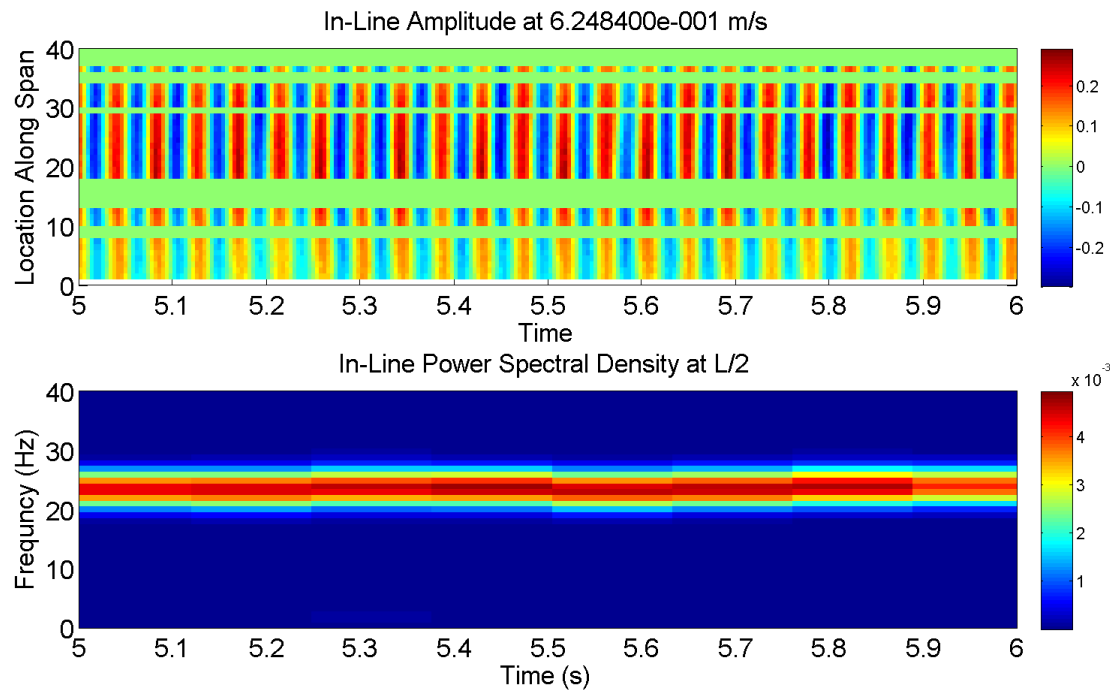

Figure D.11: $\mathrm{Vr}=5.35(\mathrm{U}=0.62 \mathrm{~m} / \mathrm{s})$ Time history of amplitude and PSD of the IL Direction
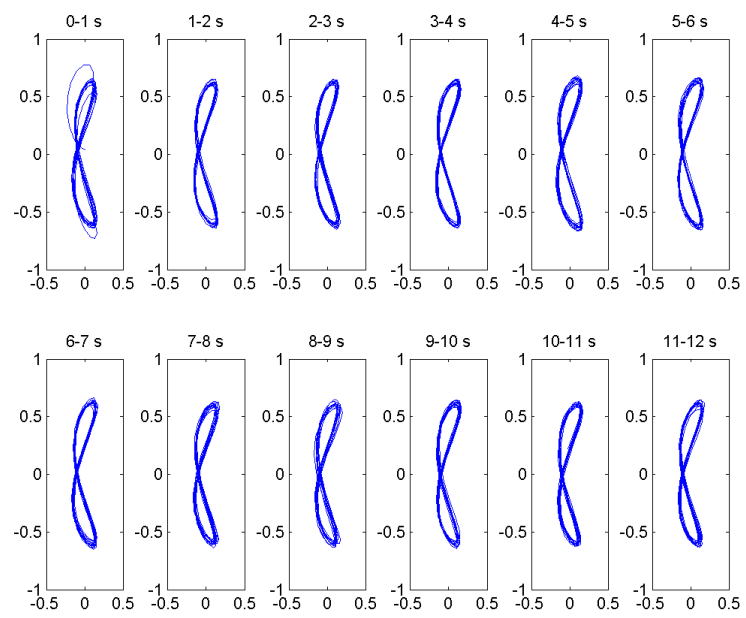

Figure D.12: $\mathrm{Vr}=5.35(\mathrm{U}=0.62 \mathrm{~m} / \mathrm{s})$ orbital patterns $0-12 \mathrm{sec}$ 


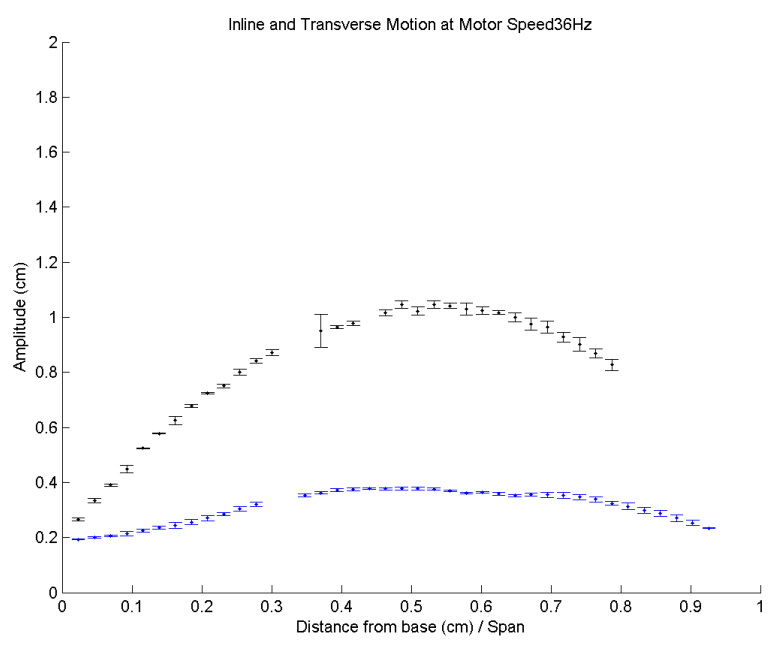

Figure D.13: $\mathrm{Vr}=5.41(\mathrm{U}=0.65 \mathrm{~m} / \mathrm{s})$ Spanwise shape of in-line (Black) and cross-flow (Blue) directions
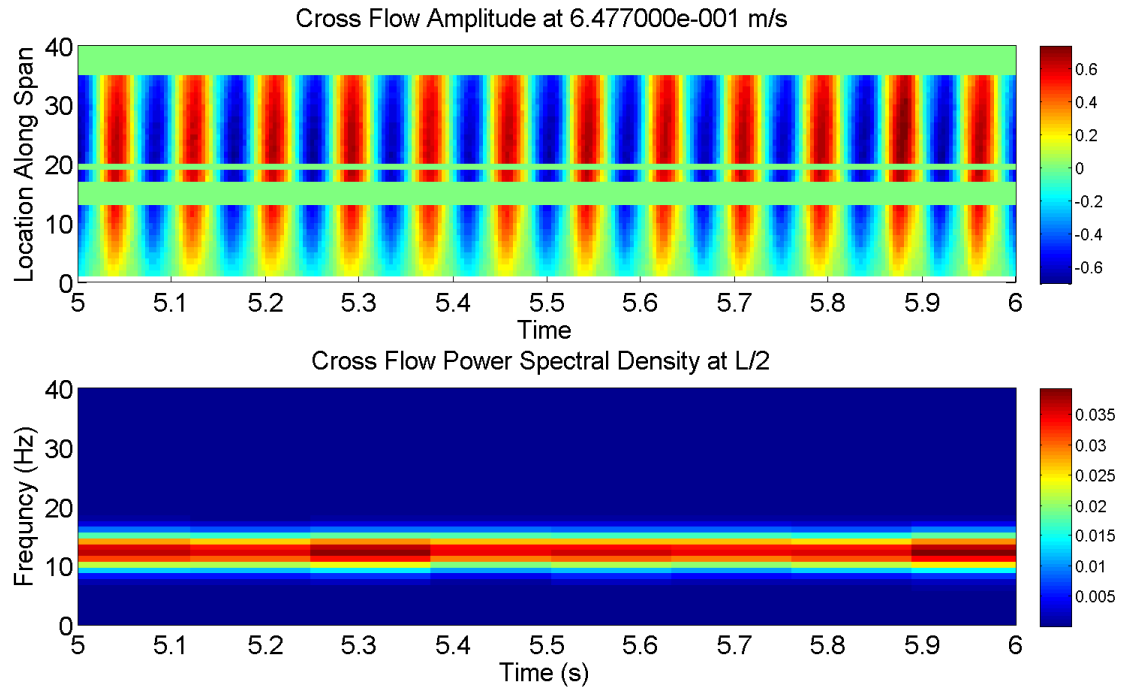

Figure D.14: $\mathrm{Vr}=5.41(\mathrm{U}=0.65 \mathrm{~m} / \mathrm{s})$ Time history of amplitude and PSD of the CF Direction 

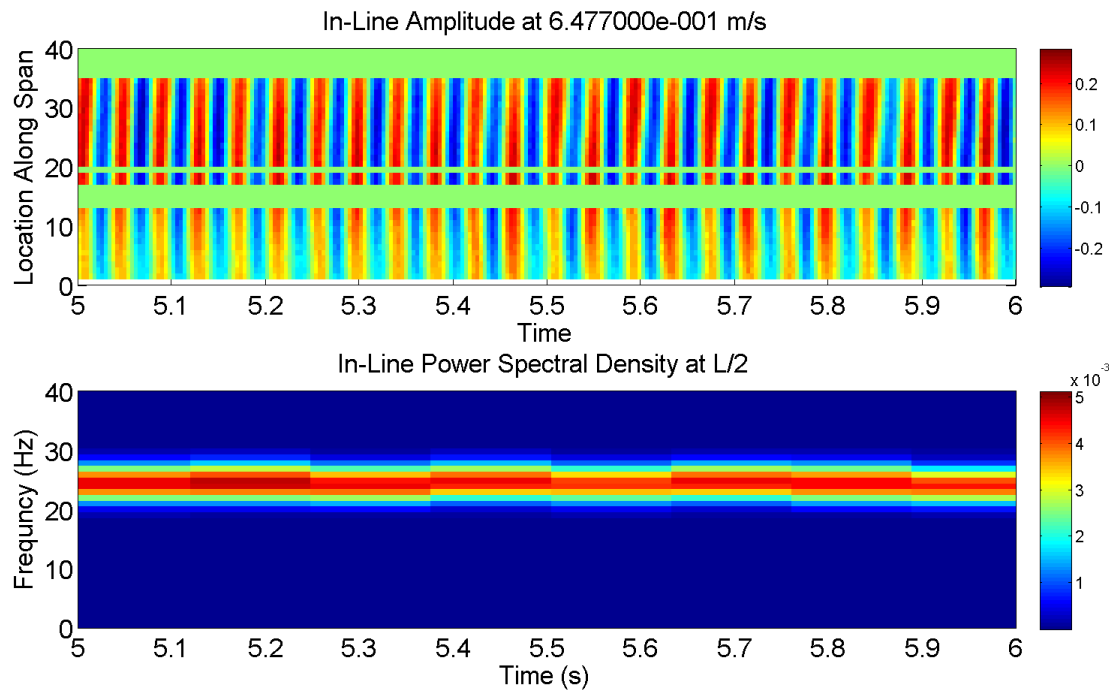

Figure D.15: $\mathrm{Vr}=5.41(\mathrm{U}=0.65 \mathrm{~m} / \mathrm{s})$ Time history of amplitude and PSD of the IL Direction
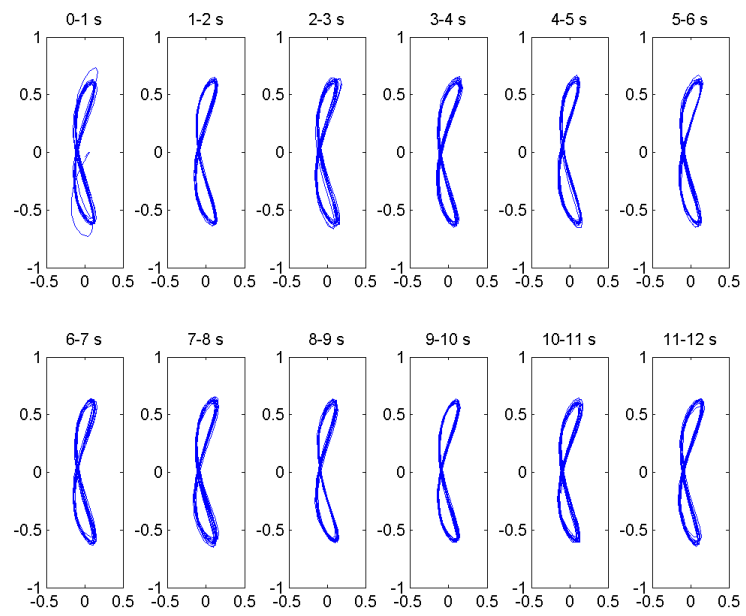

Figure D.16: $\mathrm{Vr}=5.41(\mathrm{U}=0.65 \mathrm{~m} / \mathrm{s})$ orbital patterns $0-12 \mathrm{sec}$ 


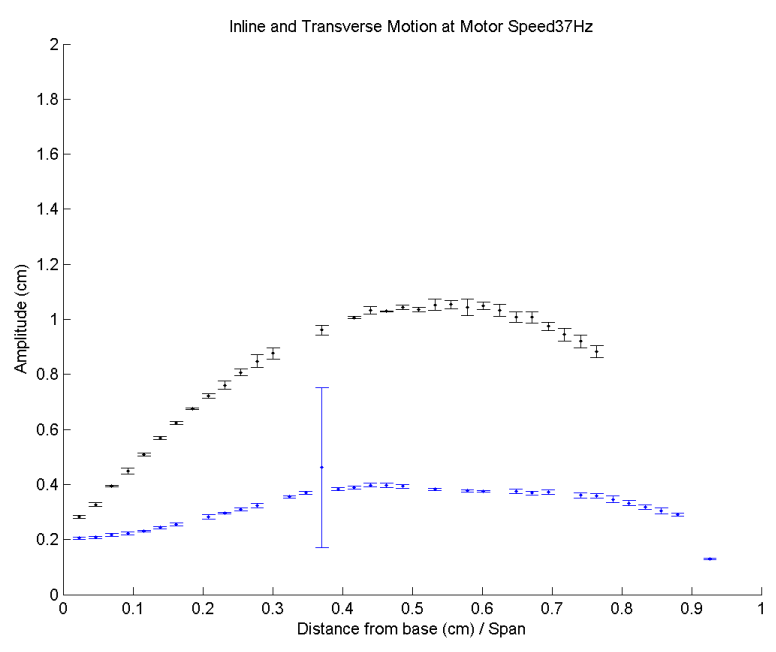

Figure D.17: $\mathrm{Vr}=5.35(\mathrm{U}=0.66 \mathrm{~m} / \mathrm{s})$ Spanwise shape of in-line (Black) and cross-flow (Blue) directions
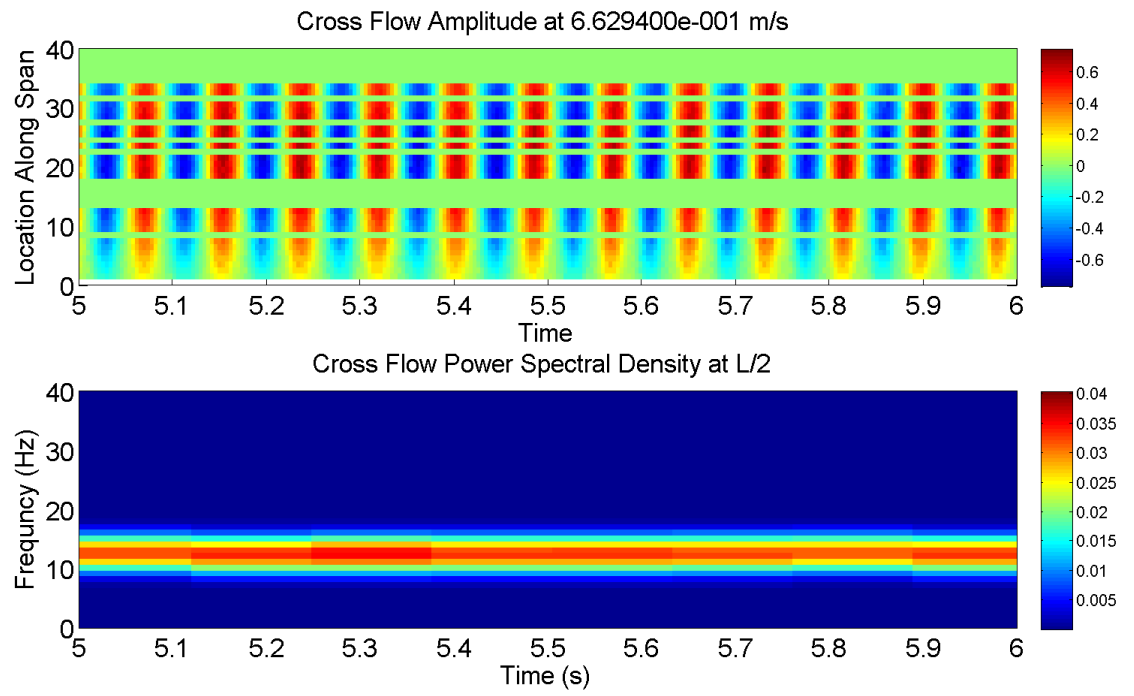

Figure D.18: $\mathrm{Vr}=5.35(\mathrm{U}=0.66 \mathrm{~m} / \mathrm{s})$ Time history of amplitude and PSD of the CF Direction 

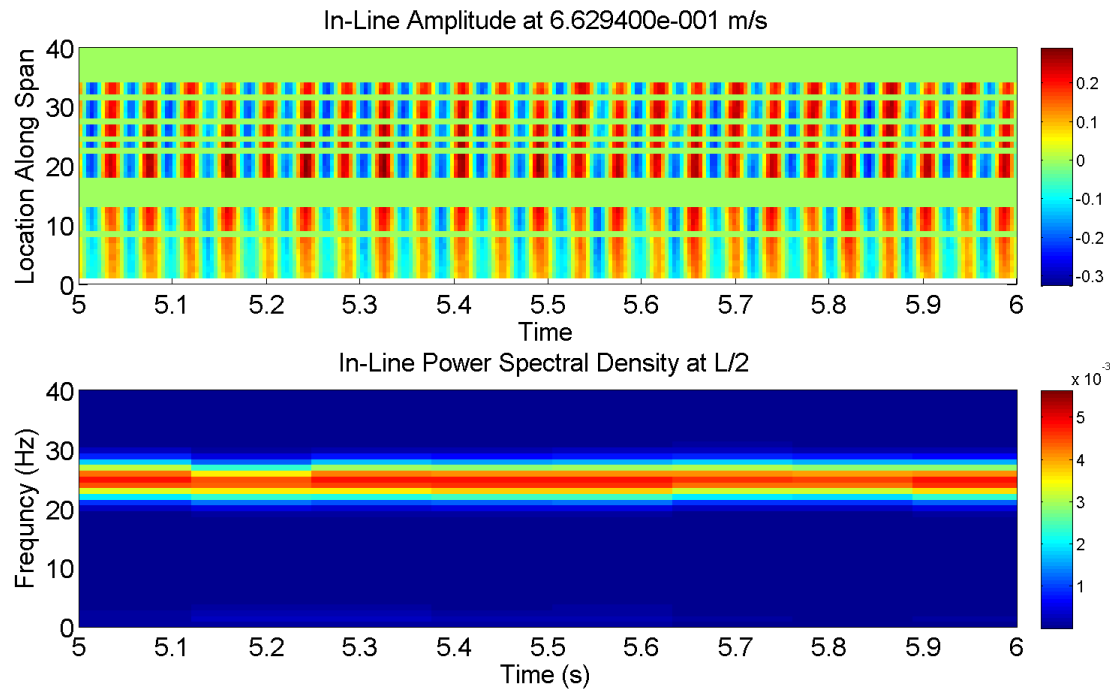

Figure D.19: $\mathrm{Vr}=5.35(\mathrm{U}=0.66 \mathrm{~m} / \mathrm{s})$ Time history of amplitude and PSD of the IL Direction

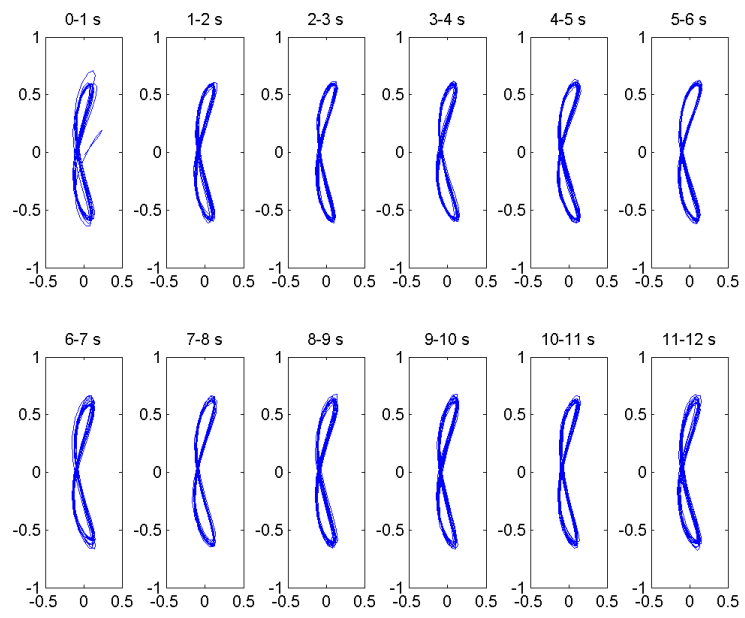

Figure D.20: $\mathrm{Vr}=5.35(\mathrm{U}=0.66 \mathrm{~m} / \mathrm{s})$ orbital patterns $0-12 \mathrm{sec}$ 


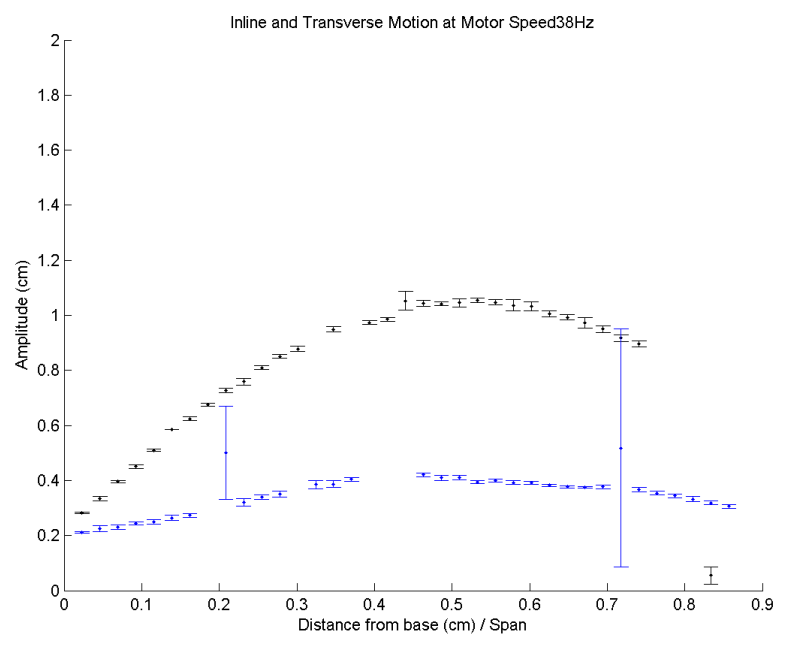

Figure D.21: $\mathrm{Vr}=5.40(\mathrm{U}=0.69 \mathrm{~m} / \mathrm{s})$ Spanwise shape of in-line (Black) and cross-flow (Blue) directions
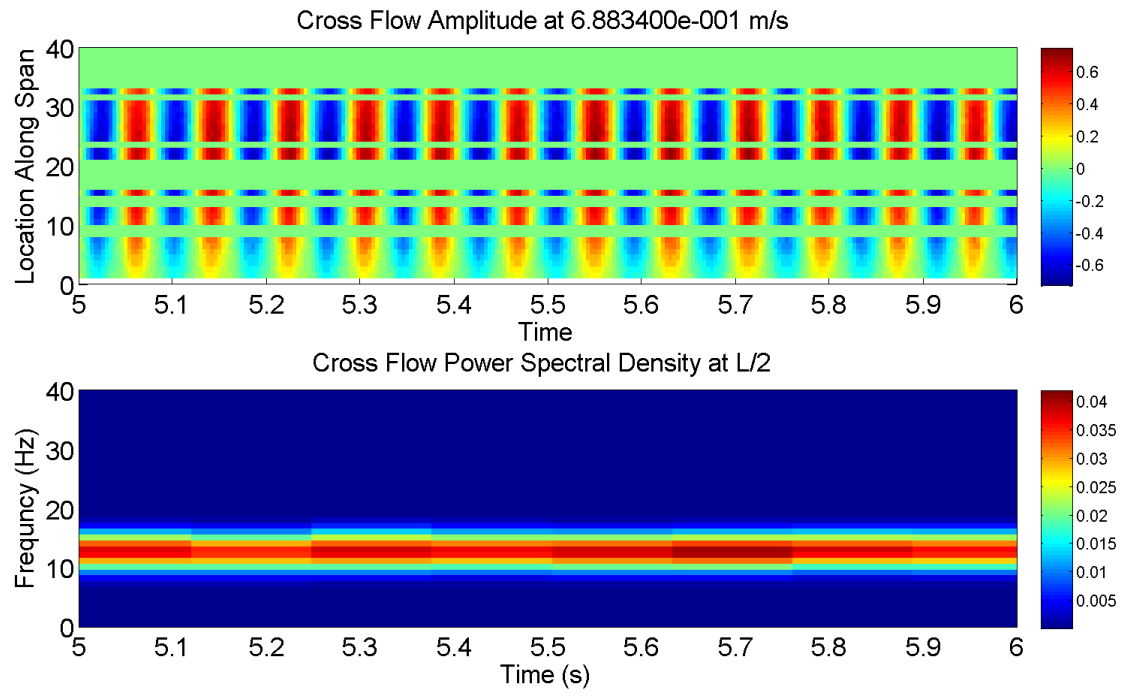

Figure D.22: $\mathrm{Vr}=5.40(\mathrm{U}=0.69 \mathrm{~m} / \mathrm{s})$ Time history of amplitude and PSD of the CF Direction 

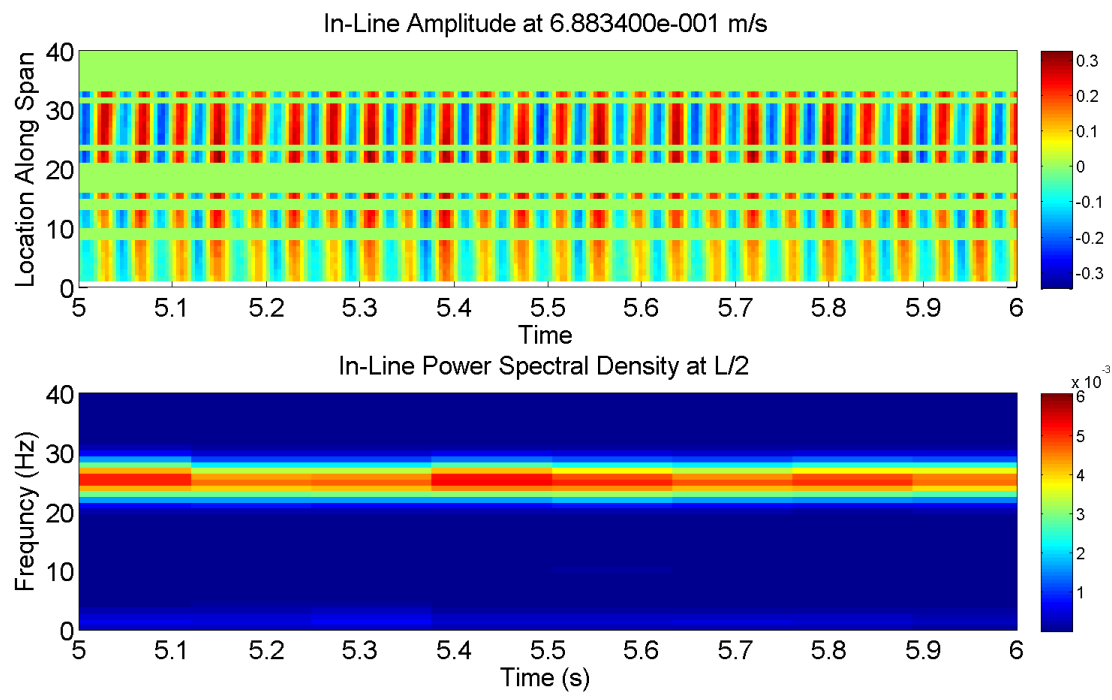

Figure D.23: $\mathrm{Vr}=5.40(\mathrm{U}=0.69 \mathrm{~m} / \mathrm{s})$ Time history of amplitude and PSD of the IL Direction
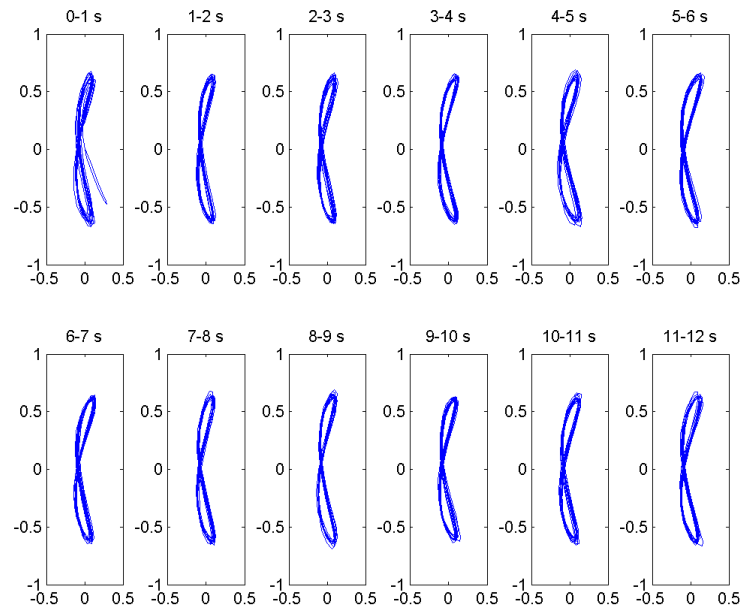

Figure D.24: $\mathrm{Vr}=5.40(\mathrm{U}=0.69 \mathrm{~m} / \mathrm{s})$ orbital patterns $0-12 \mathrm{sec}$ 


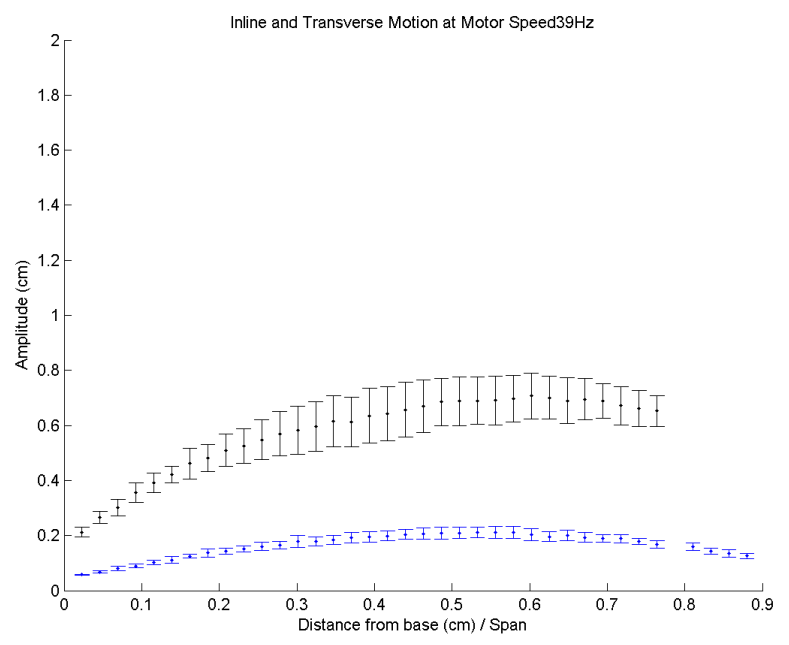

Figure D.25: $\mathrm{Vr}=5.13(\mathrm{U}=0.70 \mathrm{~m} / \mathrm{s})$ Spanwise shape of in-line (Black) and cross-flow (Blue) directions
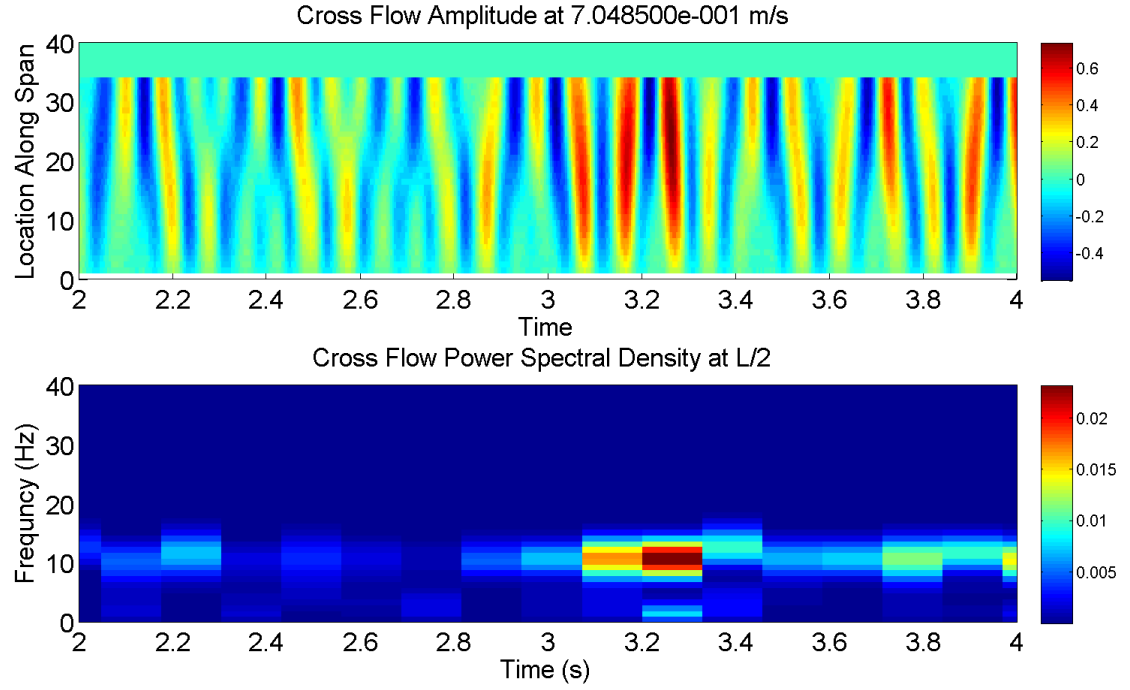

Figure D.26: $\mathrm{Vr}=5.13(\mathrm{U}=0.70 \mathrm{~m} / \mathrm{s})$ Time history of amplitude and PSD of the CF Direction 

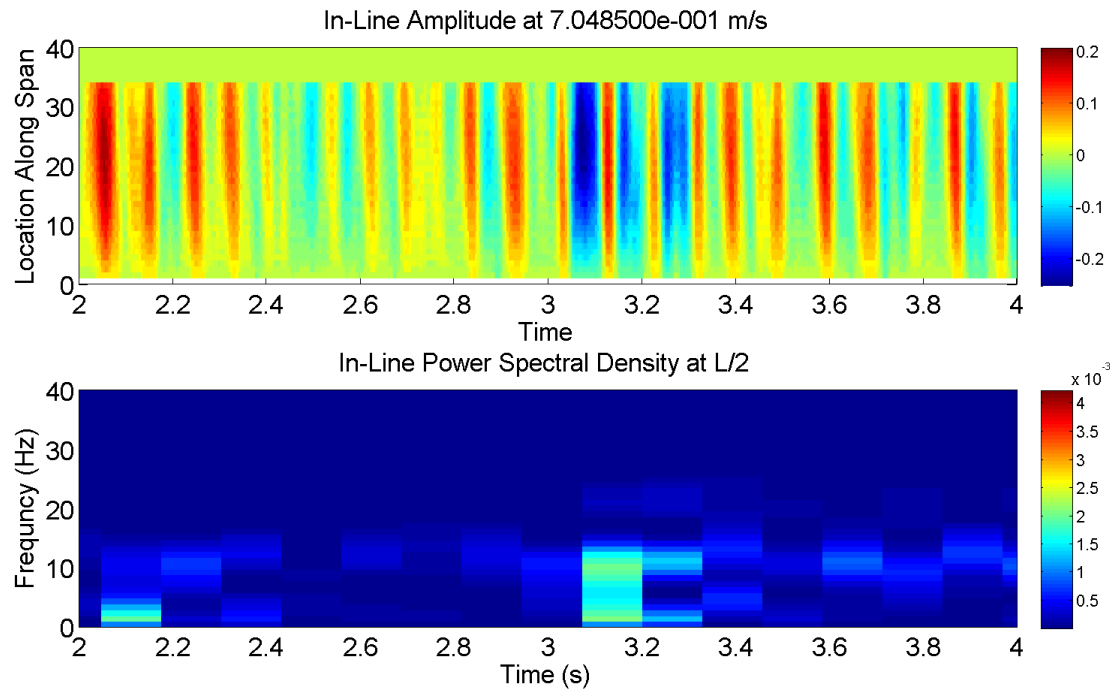

Figure D.27: $\mathrm{Vr}=5.13(\mathrm{U}=0.70 \mathrm{~m} / \mathrm{s})$ Time history of amplitude and PSD of the IL Direction
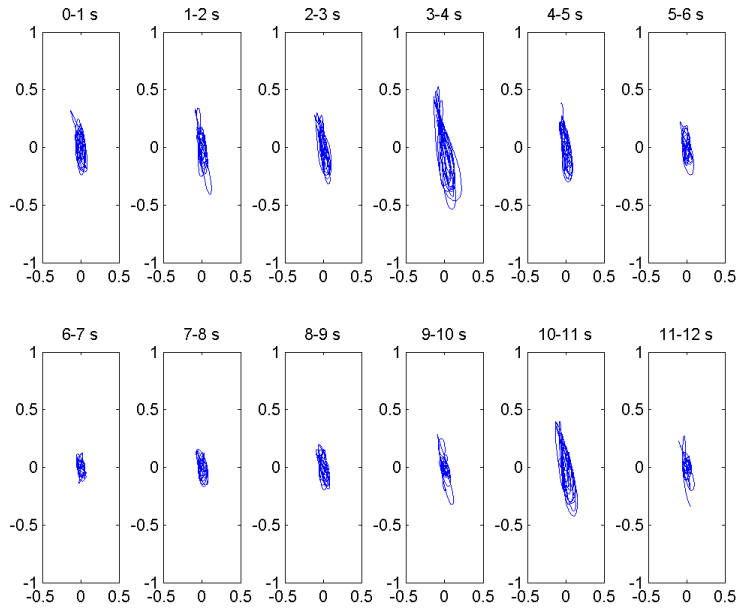

Figure D.28: $\mathrm{Vr}=5.13(\mathrm{U}=0.70 \mathrm{~m} / \mathrm{s})$ orbital patterns $0-12 \mathrm{sec}$ 


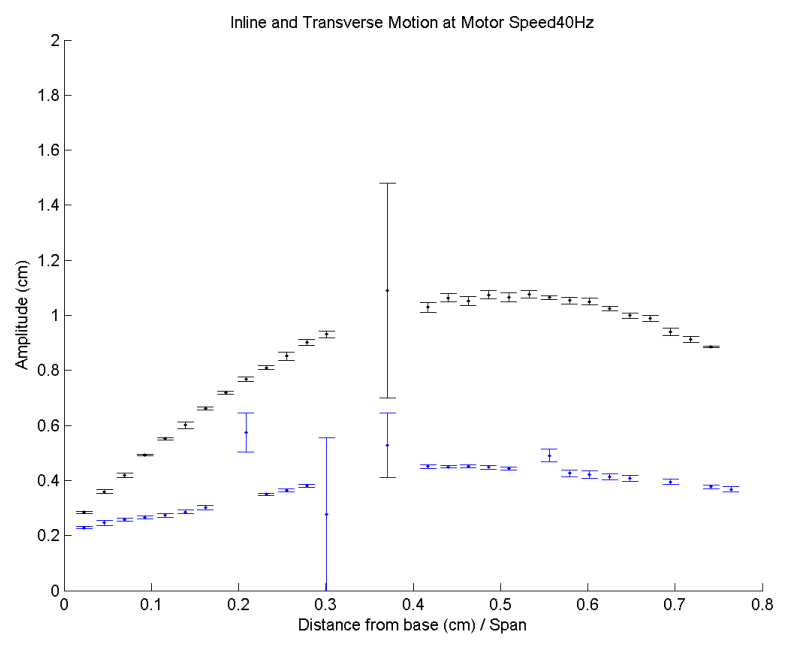

Figure D.29: $\mathrm{Vr}=5.36(\mathrm{U}=0.72 \mathrm{~m} / \mathrm{s})$ Spanwise shape of in-line (Black) and cross-flow (Blue) directions
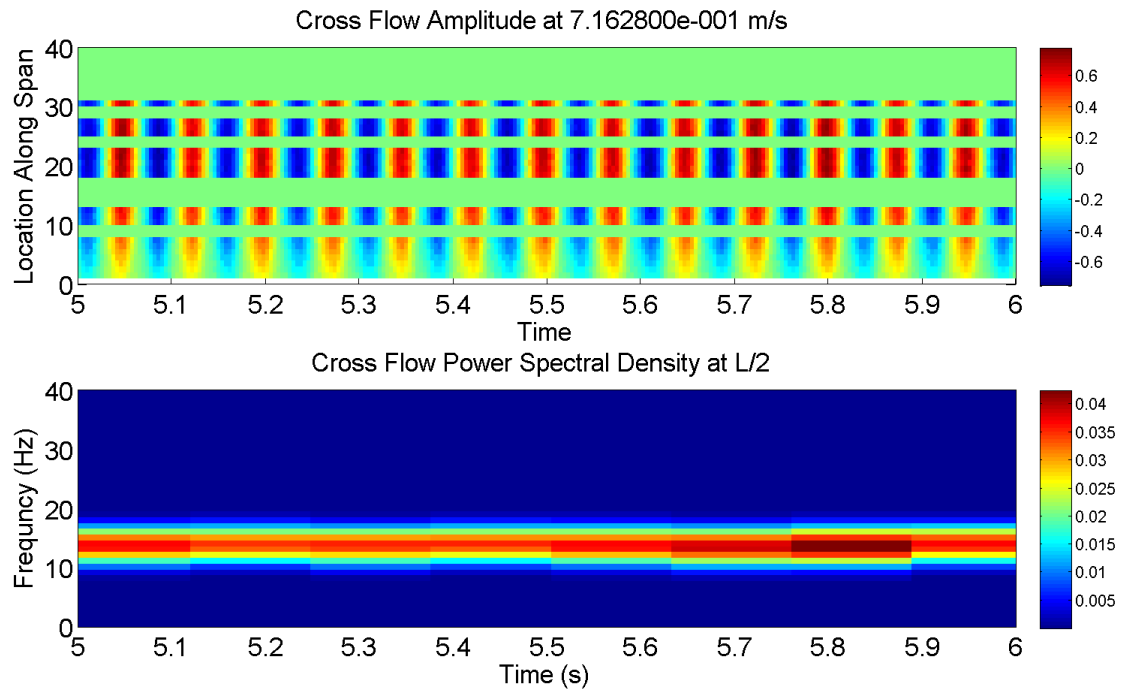

Figure D.30: $\mathrm{Vr}=5.36(\mathrm{U}=0.72 \mathrm{~m} / \mathrm{s})$ Time history of amplitude and PSD of the CF Direction 

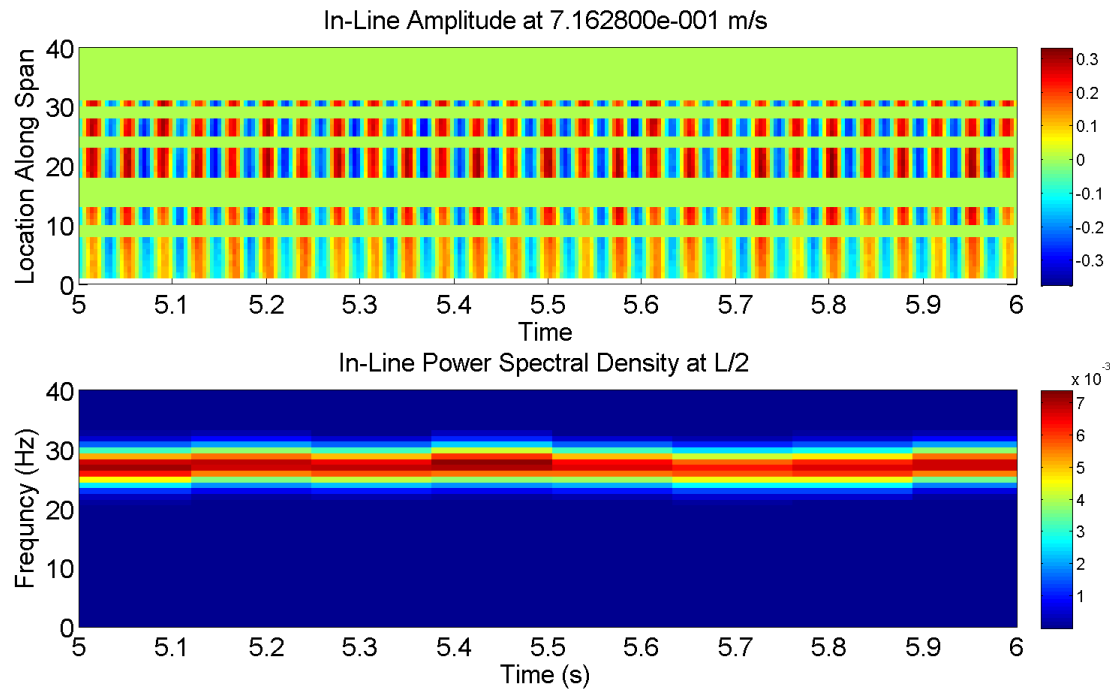

Figure D.31: $\mathrm{Vr}=5.36(\mathrm{U}=0.72 \mathrm{~m} / \mathrm{s})$ Time history of amplitude and PSD of the IL Direction
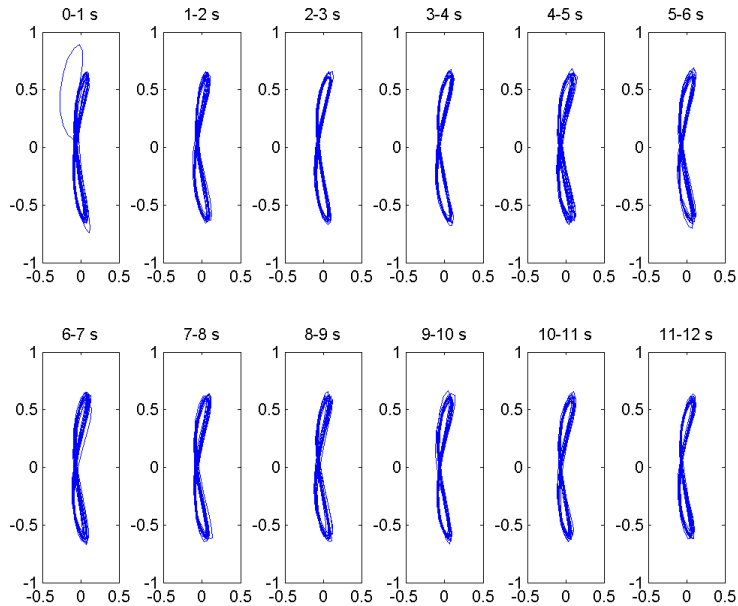

Figure D.32: $\mathrm{Vr}=5.36(\mathrm{U}=0.72 \mathrm{~m} / \mathrm{s})$ orbital patterns $0-12 \mathrm{sec}$ 


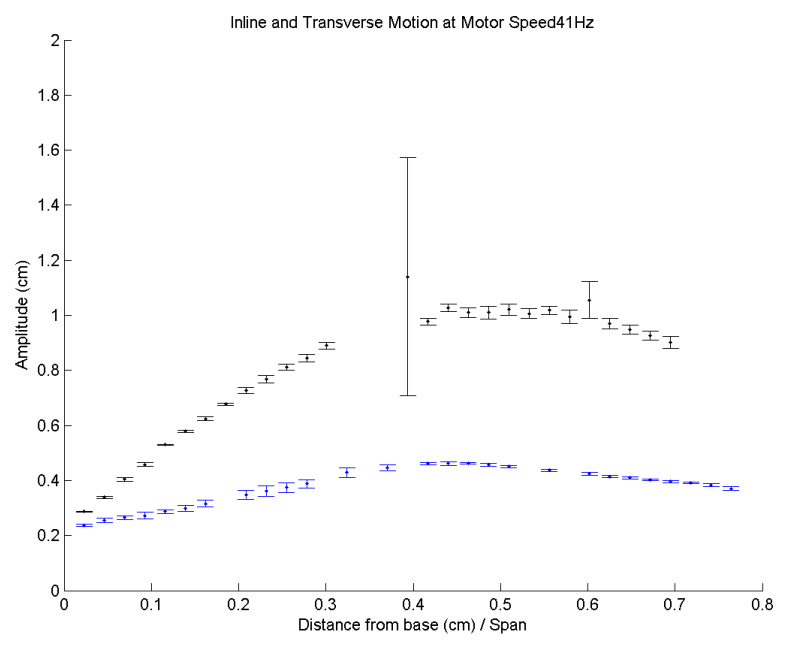

Figure D.33: $\mathrm{Vr}=5.30(\mathrm{U}=0.73 \mathrm{~m} / \mathrm{s})$ Spanwise shape of in-line (Black) and cross-flow (Blue) directions
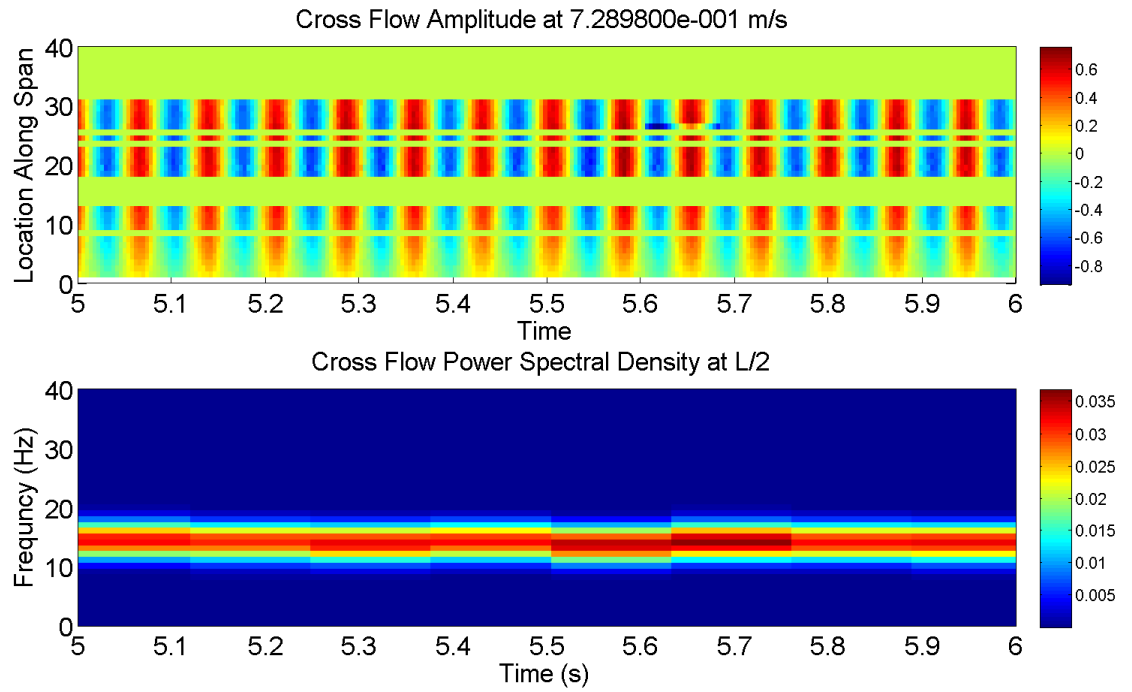

Figure D.34: $\mathrm{Vr}=5.30(\mathrm{U}=0.73 \mathrm{~m} / \mathrm{s})$ Time history of amplitude and PSD of the CF Direction 

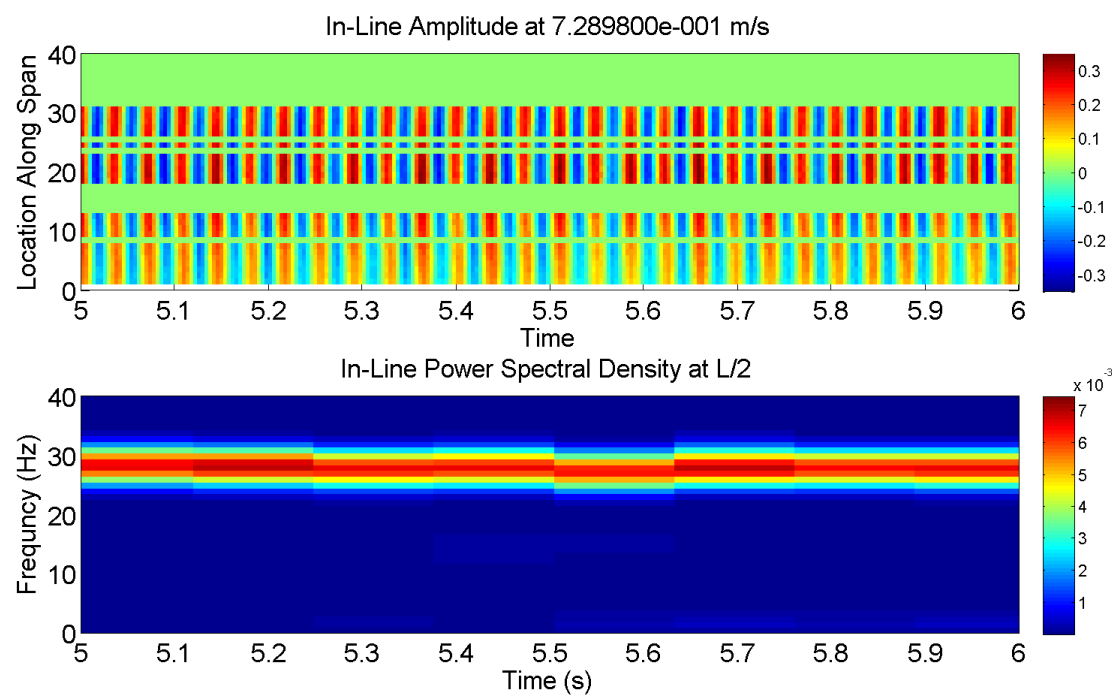

Figure D.35: $\mathrm{Vr}=5.30(\mathrm{U}=0.73 \mathrm{~m} / \mathrm{s})$ Time history of amplitude and PSD of the IL Direction
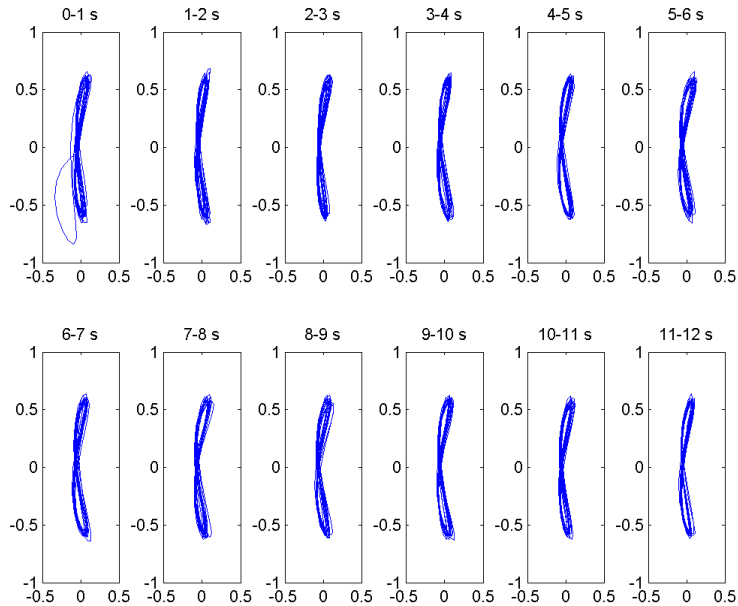

Figure D.36: $\mathrm{Vr}=5.30(\mathrm{U}=0.73 \mathrm{~m} / \mathrm{s})$ orbital patterns $0-12 \mathrm{sec}$ 


\section{BIBLIOGRAPHY}

Bearman, P., "Vortex shedding from oscillating bluff bodies," Annual Review of Fluid Mechanics, vol. 16, pp. 195-222, 1984.

Bearman, P., "Circular cylinder wakes and vortex-induced vibrations," Journal of Fluids and Structures, vol. 27, pp. 648-658, 2011.

Blevins, R., Flow Induced Vibration. New York, NY: Van Nostrand Reinhold Company, 1990.

Dahl, J., Hover, F., Triantafyllou, M., Dong, S., and Karniadakis, G., "Resonant vibrations of bluff bodies cause multi-vortex shedding," Physical Review Letters, October 2007.

Dahl, J., Hover, H., and Triantafyllou, M., "Two-degree-of-freedom vortex- induced vibrations using a force assisted apparatus," Journal of Fluids and Structures, vol. 22, pp. 807-818, 2006.

Dahl, J. M., "Vortex-induced vibration of a circular cylinder with combined inline and cross-flow motion," Ph.D. dissertation, Massachusetts Institute of Technology, June 2008.

E. Passano, C.M. Larsen, J. W., "Viv of free spinning pipelines: comparison of response from semi-empirical code to model tests," Proceedings of the ASME 2010 29th International Conference on Ocean, Offshore and Arctic Engineering, 2010.

Feng, C., "The measurements of vortex-induced effects on flow past stationary and oscillating circular and d-section cylinders." Master's thesis, University of British Columbia, 1968.

Jauvtis, N. and Williamson, C., "The effect of two degrees of freedom on vortexinduced vibration at low mass and damping," Journal of Fluid Mechanics, vol. 509, pp. 23-62, 2004.

Jeon, D. and Gharib, M., "On circular cylinders undergoing two-degree-of-freedom forced motions," Journal of Fluids and Structures, vol. 15, pp. 533-541, 2001.

Kang, Z. and Jia, L., "An experiment study of a cylinder's two degree of freedom viv trajectories," Ocean Engineering, vol. 70, pp. 129-140, 2013.

Khalak, A. and Williamson, C., "Motions, forces and mode transitions in vortexinduced vibrations at low mass-damping," Journal of Fluids and Structures, vol. 13, pp. 813-851, 1999. 
King, R., "Vortex-excited oscillations of a circular cylinder in steady currents." in Paper OTC 1948. Houston, TX, USA: Offshore Technology Conference, 1974.

King, R., "A review of vortex shedding research and its application," Ocean Engineering, vol. 4, no. 3, pp. 141-171, 1977.

Moe, G. and Wu, Z.-J., "The left force on a cylinder vibrating in a current," Journal of Offshore Mechanics and Arctic Engineering, vol. 112, pp. 297-303, November 1990.

Newman, J., Marine Hydrodynamics. Cambridge, MA: The MIT Press, 1977.

Norberg, C., "Fluctuating lift on a circular cylinder: Review and new measurements," Journal of Fluids and Structures, vol. 17, pp. 57-96, 2003.

Parkinson, G., "Mathematical models of flow-induced vibrations of bluff bodies," Flow-induced structural vibrations, 1974.

Richardson, French, G., L.S., B., and et al, R., "Deep water gulf of mexico 2044: America's expanding frontier," US Department of Interior, Mineral Management Service, Tech. Rep., 2004.

Sarpkaya., T., "Vortex induced oscillations." Journal of Applied Mechanics, vol. 46, pp. 241- 258, 1979.

Sarpkaya, T., "Hydrodynamic damping, flow-induced oscillations, and biharmonic response," ASME Journal of Offshore Mechanics and Arctic Engineering, vol. 117, pp. 232-238, 1995.

Sarpkaya, T., "A critical review of the intrinsic nature of vortex-induced vibrations." Journal of Fluids and Structures, vol. 19, pp. 389-447, 2004.

Sumer, B. M. and Fredsoe, J., Hydrodynamics Around Cylindrical Structures, revised edition ed., ser. Advanced Series on Ocean Engineering. Singapore: World Scientific, 2006, vol. volume 26.

Trim, A., Braaten, H., Lie, H., and Tognarelli, M., "Experimental investigation of vortex-induced vibration of long marine risers," Journal of Fluids and Structures, vol. 21, no. 335-361, 2005.

Vandiver, J., "Drag coefficients of long flexible cylinders." Texas, USA: Offshore Technology Conference, May 1983.

Vandiver, J. and Jong, J., "The relationship between in-line and cross-flow vorex induced vibration of cylinders," Journal of Fluids and Structures, vol. 1, pp. 381-399, 1987. 
VERITAS, D. N., "Dnv-os-c101," April 2011.

Williamson, C. and Govardhan., R., "Vortex-induced vibrations," Annual Review of Fluid Mechanics, vol. 36, pp. 413-455, 2004. 